Sterrch Meet's Biofechnology

In Planta Modificalition of Starch Composition and Functionalitijes

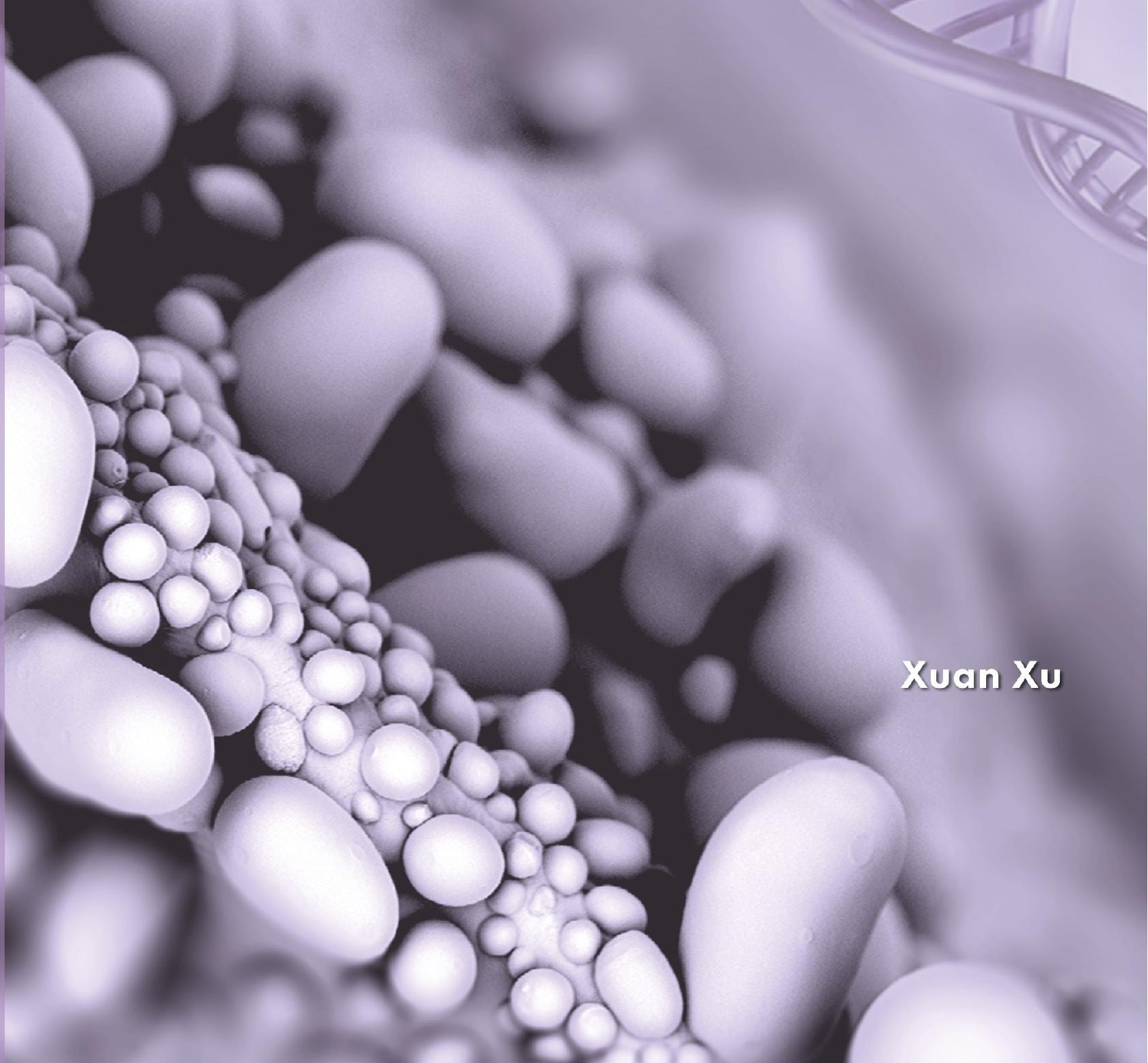





\section{Starch Meets Biotechnology}

\section{In Planta Modification of}

Starch Composition and Functionalities 


\section{Thesis committee}

\section{Promotor}

Prof. Dr R.G.F. Visser

Professor of Plant Breeding

Wageningen University \& Research

\section{Co-promotor}

Dr L.M. Trindade

Associate professor, Wageningen UR Plant Breeding

Wageningen University \& Research

\section{Other members}

Prof. Dr H.A. Schols, Wageningen University

Prof. Dr M.J.E.C. van der Maarel, University of Groningen

Dr J.C.P. Hopman, Averis Seeds B.V., Valthermond

Dr C.G. Boeriu, Wageningen University \& Research

This research was conducted under the auspices of the Graduate School of Experimental Plant Sciences 


\title{
Starch Meets Biotechnology \\ In Planta Modification of \\ Starch Composition and Functionalities
}

\author{
Xuan Xu
}

Thesis

submitted in fulfilment of the requirements for the degree of doctor

at Wageningen University

by the authority of the Rector Magnificus

Prof. Dr A.P.J. Mol,

in the presence of the

Thesis Committee appointed by the Academic Board

to be defended in public

on Friday 14 October 2016

at 11 a.m. in the Aula. 


\section{Xuan Xu}

Starch Meets Biotechnology - In Planta Modification of Starch Composition and Functionalities

170 pages.

PhD thesis, Wageningen University, Wageningen, NL (2016)

With references, with summary in English

ISBN 978-94-6257-920-0

DOI 10.18174/389540 


\section{Table of Contents}

$\begin{array}{lll}\text { Chapter } 1 & \text { General introduction } & 7\end{array}$

Chapter 2 Starch modification by biotechnology: 21 state of art and perspectives

Chapter 3 Engineering Potato Starch with a Higher Phosphate Content

Chapter 4 Starch phosphorylation plays an important role in starch biosynthesis

Chapter 5 Introduction and Expression of Arabidopsis Starch Dikinases in 87 Potato

Chapter 6 Expression of an (engineered) 4,6-a-glucanotransferase in potato results in changes in starch characteristics

Chapter 7 General Discussion

References

Summary

Acknowledgements

About the author 165

Overview of training activities 



\section{Chapter 1}

General Introduction 


\section{Starch, an attractive bio-resource}

Starch is the most widespread and predominant storage carbohydrate in higher plants, which can provide energy for plant development. It is synthesized from assimilated sugars and assembled into a granular form in specific tissues. In the leaves of plants, starch serves as short-term energy reserve to ensure continued supply of sugars in dayto-day carbohydrate metabolism. This type of starch is accumulated during the daytime photosynthesis and remobilized subsequently during the night and is thus named "transitory starch". On the other hand, starch accumulated in heterotrophic organs (e.g. seed, roots and tubers) is termed "storage starch", as this type of starch serves as a longterm energy source to fuel regrowth upon sprouting or germination. Additionally, and importantly, storage starch plays a multifunctional role in human consumption, serving as a primary source of dietary calories in human diet and also as an inexpensive, renewable and biodegradable material in a broad range of industrial applications.

Due to the wide uses of starch, the global production of starch containing crops outweighs other industrial and food crops. At present, the main sources of storage starch are from maize, cassava, wheat and potatoes, which are mainly produced by China, North America, Europe, Southeast Asia and South America (Food and Agriculture Organization of the United Nations, http://faostat3.fao.org/home/E). The most important outlet by far of all starch-derived products is relevant to food; however, the consumption of starch has greatly increased with starch becoming an important component for bio-fuels, paper and glue production, textile weaving and finishing and the fermentation industry. To illustrate, in Europe, the total production of starch in 2014 was 10.5 million tons with $47.5 \%, 39.2 \%$ and $13.3 \%$ for maize, wheat and potatoes, respectively, which was increased by $21 \%$ compared to 2004 (European Starch Industry Association, www.aaf-eu.org). These starches were produced from in total 23 million tons of agricultural materials, which consist of 16 million tons of cereals and 7 million tons of starch potatoes (Fig. 1a and b). Of this amount, the largest single outlet was (has always been) for sweeteners production (Fig. 1c) and was mainly consumed in confectionary and drinks (Fig. 1d).

The global starch market has continuously expanded over the years. It is expected to exceed 150 million metric tons by 2020 driven by the increasing population, cost benefits and sustainable consumption patterns. Correspondingly, the global sales of starches and derivatives are projected to reach $\$ 77.4$ billion by 2018, up from $\$ 51.2$ billion in 2012 (BCC research, http://www.bccresearch.com/market-research/food-and-beverage/starchglucose-fod037b.html). Nonetheless, a big challenge ahead is that the rapid market expansion conflicts with a shortening availability of suitable raw materials at tolerable prices and increasing energy costs. 
To overcome this challenge and to meet this rapid growing demand from a growing human population in a changing climate, producing starch with high quality in large quantities through a more innovative, cost-effective, and sustainable manner is inevitable and warranted. At present, while much of the researches are focusing on productive output, an increasing number of studies has emerged to enhance functional characteristics of starches, aiming at producing starches that are more convenient to distribute and easier to process and have specific physico-chemical properties required by the target markets. These value-added starches can provide society with high-value raw materials to satisfy market needs, thereby broadening the path towards a robust starch industry and a truly bio-based and circular economy.

\section{(a) Processed raw materials}

Total: 23 million tons

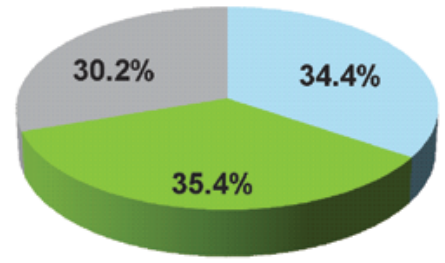

(c) Consumption of starch \& starch derivatives

Total: 9 million tons

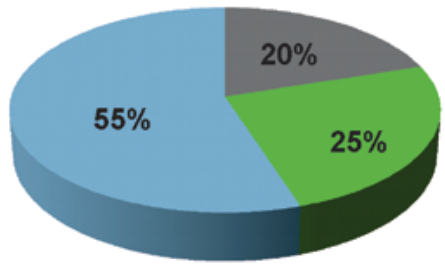

$$
\begin{aligned}
& \text { Modified starches } \\
& \text { Native starches } \\
& \text { Starch sweeteners }
\end{aligned}
$$

\section{(b) Starch products in starch equivalent}

Total: 10.5 million tons

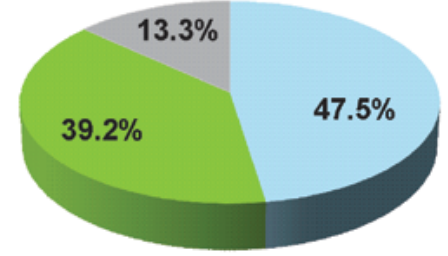

(d)

\section{$\underline{\text { Starch applications }}$}

Total: 9 million tons

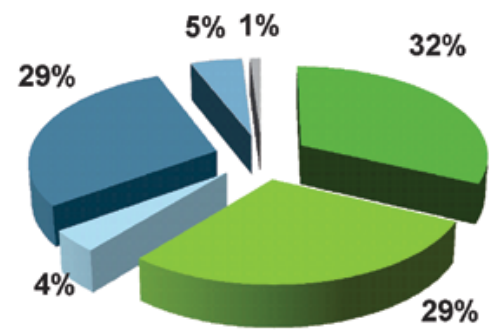

\begin{tabular}{|ll}
\hline Confectionery and Drinks & Corrugating and Paper \\
Other food & Other Non-food \\
Pharma \& Chemicals & Feed
\end{tabular}

FIGURE 1. Overview of European starch production and consumption in 2014. Source: European Starch Industry Association, www.aaf-eu.org. 


\section{Green technology, genetic modification, is needed for starch modification}

\section{Genetic modification of starch, an inexorable trend}

The competitive advantage and commercial success of starches is mainly determined by their functional characteristics. Surprisingly, most of the native starches are inherently not suitable for industrial applications, notwithstanding the large variations in their functional properties. This is mainly due to the high and unstable viscosity of the cooked starch, retrogradation and gelling tendencies of starch pastes. Therefore, starches are often modified to eliminate deficiencies and achieve the more appropriate properties for different industrial applications, which in turn enhances the starch's versatility and satisfies the consumers' demands.

Several methods of starch modification are used in industry: chemical, physical and enzymatic modification. Chemical modification is one common method to modify starches for industry. Using various chemical reagents, chemical moieties can be added on the a-D-glucopyranosyl units of starches by molecular scission, oxidation, esterification, etherification or molecular arrangements (Chiu and Solarek, 2009; Shrestha and Halley, 2014), thereby enhancing the functionality of starch. Additionally, physical modification is also widely used in industries to generate pregelatinized, milled, and cold-water-soluble starches through drum drying, spray drying, extrusion cooking, annealing and heatmoisture treatment (Haghayegh and Schoenlechner, 2011). Enzymatic modification is another strategy that evolved not long ago and hydrolyzing enzymes are mainly used to modify starch for industrial applications. The products are very suitable for coating food items with seasonings, flavors and colorants, as they retain a good adhesion property during hydrolysis, which can be applied at high solids to minimize the energy required to remove moisture after application (Chiu and Solarek, 2009). All in all, these methods have largely extended the end uses of starch; however, they are expensive, time-consuming and frequently generate hazardous wastes.

Our society is becoming more and more aware that producing modified starch through these conventional methods could not fully meet the requirements for environmental and economic sustainability. Thus, large-scale research has been executed to explore green technologies, and one of them is genetic modification of starch in planta. This approach allows us to generate tailor-made starches in plants that are directly suitable for a particular industrial use, thereby circumventing expensive and environment-unfriendly post-harvest processes and thus providing tremendous economic and environmental advantages. In other words, genetic modification of starches provides opportunities to produce novel starches with enhanced properties in a sustainable and economical manner, which is an inexorable trend for realizing a robust starch industry. 


\section{Strategies for genetic modification of starch}

Our understanding of starch biosynthesis was greatly improved with studies on transitory starch in Arabidopsis thaliana, which provides a bulk of information for targeted design of novel starches. However, the main challenge is to apply the acquired knowledge in economically important starch crops for improving starch quality. In the past decades, continuous efforts have been made to realize this purpose. There are two main strategies to genetically modify starch in planta: one is to modulate endogenous genes involved in starch biosynthesis; another is to express heterologous genes encoding biosynthetic or modifying enzymes from other organisms. Using the first strategy, the activity of one crucial gene or multiple genes can be manipulated to generate starch with various characteristics in crop plants, such as starch with differences in amylose-to-amylopectin ratio, starch structure, phosphate content and granule size (more details in Chapter 2, section 5).

On the other hand, introduction of enzymes from other organisms has enormous potential to diversify characteristics of starch thus creating novel properties. Many achievements have been gained by introducing starch-modifying bacterial enzymes into crops including potato, maize and cassava (more details in Chapter 2, section 4). Additionally, this strategy can also be used to produce novel polymers (e.g. mutan, dextran and alternan) in amyloplasts, which is beneficial to generate commercial added value (Moire et al., 2003) (more details in Chapter 2, section 4).

To achieve efficient starch bioengineering in planta and gain stronger effects on starch properties, the so-called Starch Binding Domain (SBD) technology was developed (Ji et al., 2003). It was shown that SBD exists in a wide range of hydrolases and has the affinity to bind to starch granules. By fusing the target enzyme (with no affinity for starch on their own) to $S B D$, the fusion enzyme can be targeted to starch granules during starch biosynthesis, and by that facilitate the access of the enzyme to the substrate thus enhancing the enzyme action. This makes SBD a potential tool to modify starch in planta and several potato starches with novel characteristics have been successfully generated by using this technology (Firouzabadi et al., 2007; Huang et al., 2013; Kok-Jacon et al., 2005a). One of the successful examples was to anchor amylosucrase from Neisseria polysaccharea in starch granules by using this technology, which led to altered starch granule morphology, bigger granule size and higher starch digestibility (Huang et al., 2014). In this thesis, SBD technology was applied in studies to target enzymes to starch granules during starch biosynthesis, thereby facilitating the action of enzymes and thus enhancing the effect on starch structure and functionality. 


\section{Potato tuber, a superior model system for storage starch biosynthesis studies and for novel starch production}

Starches from different botanical sources differ considerably in many aspects (Table 1). Although the production of potato starch is relative small compared to the total amount of starch produced in the world, potato starch is often favored in many specific applications because of its unique properties and outstanding performance in processing resulting from its large granule size, low protein and lipid content, high phosphate content and high degree of amylose and amylopectin polymerization. Therefore, an efficient and economical production of potato starch with improved functionality is of great interest.

Undoubtedly, studies in the model plant Arabidopsis thaliana alone do not provide adequate information for understanding starch metabolism in crop plants. Attempts to improve starch quality were hampered by the gaps in knowledge between the biosynthesis of transitory and storage starches (Lloyd and Kossmann, 2015) and the lack of a complete understanding of the factors and mechanisms regulating the starch biosynthesis pathway in crop plants. Therefore, studies in a model system for starch crops are crucial. In this respect, potato tuber is an outstanding model for the study of storage starch metabolism (Geigenberger et al., 2004). Firstly, it is a relatively homogenous tissue in which conversion of sucrose to starch represents the dominant metabolic flux. Secondly, many key players involved in major steps of starch metabolism in potato have been identified and well characterized (Kruger, 1990), providing a good foundation to further exploit key players and investigate the biosynthesis of storage starch. Thirdly, potato mediated transformation by Agrobacterium tumefaciens has been well developed, making it easy to study effects of individual genes on starch biosynthesis and starch characteristics. For all these reasons, in this thesis, endeavors were made by using potato as a model system to produce starches with novel properties and to study storage starch metabolism. 
TABLE 1. Comparison of some native starches

\begin{tabular}{|c|c|c|c|c|c|}
\hline \multicolumn{2}{|l|}{ Characteristics } & Potato & Maize & Wheat & Cassava \\
\hline \multicolumn{2}{|l|}{ Type } & Tuber & Cereal & Cereal & Root \\
\hline \multicolumn{2}{|l|}{ Granule shape } & Oval, spherical & Round, polygonal & Round, lenticular & Oval, truncated \\
\hline \multicolumn{2}{|c|}{ Granule size $(\mu \mathrm{m})$} & $5-100$ & $2-30$ & $0.5-45$ & $4-35$ \\
\hline \multicolumn{2}{|c|}{ Phosphate $(\%, w / w)$} & 0.08 & 0.02 & 0.06 & 0.01 \\
\hline \multicolumn{2}{|l|}{ Protein $(\%, w / w)$} & 0.06 & 0.35 & 0.4 & 0.1 \\
\hline \multicolumn{2}{|l|}{ Lipid (\%, w/w) } & 0.05 & 0.7 & 0.8 & 0.1 \\
\hline \multicolumn{2}{|c|}{ Pasting temperature $\left({ }^{\circ} \mathrm{C}\right)$} & $60-65$ & $75-80$ & $80-85$ & $60-65$ \\
\hline \multicolumn{2}{|c|}{ Swelling power at $95^{\circ} \mathrm{C}$} & 1153 & 24 & 21 & 71 \\
\hline \multicolumn{2}{|c|}{ Solubility at $95^{\circ} \mathrm{C}(\%)$} & 82 & 25 & 41 & 48 \\
\hline \multicolumn{2}{|c|}{ Starch paste viscosity } & Very high & medium & low & high \\
\hline \multicolumn{2}{|l|}{ Paste texture } & Long & Short & Short & long \\
\hline \multicolumn{2}{|l|}{ Paste clarity } & Almost clear & Opaque & Cloudy & Quite clear \\
\hline \multicolumn{2}{|c|}{ Retrogradation rate } & medium & high & high & low \\
\hline \multicolumn{2}{|c|}{ Film clarity and gloss } & high & low & low & high \\
\hline \multicolumn{2}{|l|}{ Film strength } & high & low & low & high \\
\hline \multicolumn{2}{|l|}{ Film flexibility } & high & low & low & high \\
\hline \multicolumn{2}{|l|}{ Film solubility } & high & low & low & high \\
\hline \multirow{4}{*}{$\begin{array}{l}\text { Performance } \\
\text { in applications }\end{array}$} & Food & +++ & + & + & ++ \\
\hline & Paper & +++ & ++ & + & +++ \\
\hline & Textile & ++ & ++ & ++ & +++ \\
\hline & Adhesives & +++ & ++ & + & ++ \\
\hline
\end{tabular}

Table was adapted and modified from Grommers and Van Der Krogt (2009) and Jobling (2004) .

\section{Modification of starch structure and phosphate content are promising paths to create starches with novel properties}

There are many possible targets for engineering starches for novel properties in planta, since starch properties are affected by many factors. In this thesis, we have focused on modifying potato starch structure and phosphate content due to their great effects on starch properties. Meanwhile we strongly believe that these studies can help us to fill in some gaps in our understanding of starch granule formation and reversible starch phosphorylation in storage organs. 


\section{Starch structure and genetic modification of it}

\section{Starch is simple in composition, but complex in structure}

Starch consists of two types of glucose polymers; amylose and amylopectin. Amylose is essentially a linear polymer consisting of long backbone chains of glucoses linked together by a-1, 4-linkages and slightly branched by a-1, 6-linkages. It can reach a degree of polymerization (DP) of up to 4,000 glucose units and constitutes about $20-30 \%$ of most natural starches. The largest component of the starch granule is amylopectin, a highly branched polymer which contains up to 10,000 glucose units joined by a-1, 4-linkages and approximately 5\% a-1, 6 branch linkages (Pfister and Zeeman, 2016). Apart from these two major polymers, starch contains other minor components, including lipids, proteins and phosphate esters. The concentration of these components varies among plant species (Grimaud et al., 2008; Tanackovic et al., 2014a).

In contrast to the simple chemical nature of starch, the molecular organization of starch and granule architecture is considerably more intricate. Various levels of organization, ranging from unorganized areas to supermolecular structures, were found within starch granules (Fig. 2a). Surprisingly, the distribution of branch points in amylopectin chains is one of the determinants for starch granule assembly, which permits neighboring linear chains to form parallel left-handed double helices and pack into crystalline lamellae. According to the packing density of double helices, starch granules can be distinguished as dense A-type polymorph (e.g. maize), less dense B-type polymorph (e.g. potato) or both A- and B-type of polymorph (e.g. pea) (Imberty et al., 1988; Imberty and Perez, 1988).

On the other hand, the regions enriched with less densely packed chains constitute amorphous lamellae in which branch points are accumulated. Crystalline and amorphous lamellae alternate with each other and generate clusters with a periodicity of $\sim 9 \mathrm{~nm}$, which is a universal feature of starch granules regardless of botanical origin (Jenkins et al., 1993b; Zeeman et al., 2002). On the next structure level, these alternating lamellae form concentric and semi-crystalline zones within the granule and further alternate with amorphous zones that have a lower degree of order and contain much of the amylose molecules, thereby showing "growth ring" patterns with a period of a few hundred nanometers in the granules (Buttrose, 1960; Pilling and Smith, 2003).

It is likely that the organization of amylopectin chain to build the granule matrix is a physical process (Zeeman et al., 2010). The occurrence of such a delicate structure indicates the presence of complex biosynthetic machinery. A thorough understanding of the role of key players in this machinery and the relationships between them is crucial for targeted design of starch in planta. 


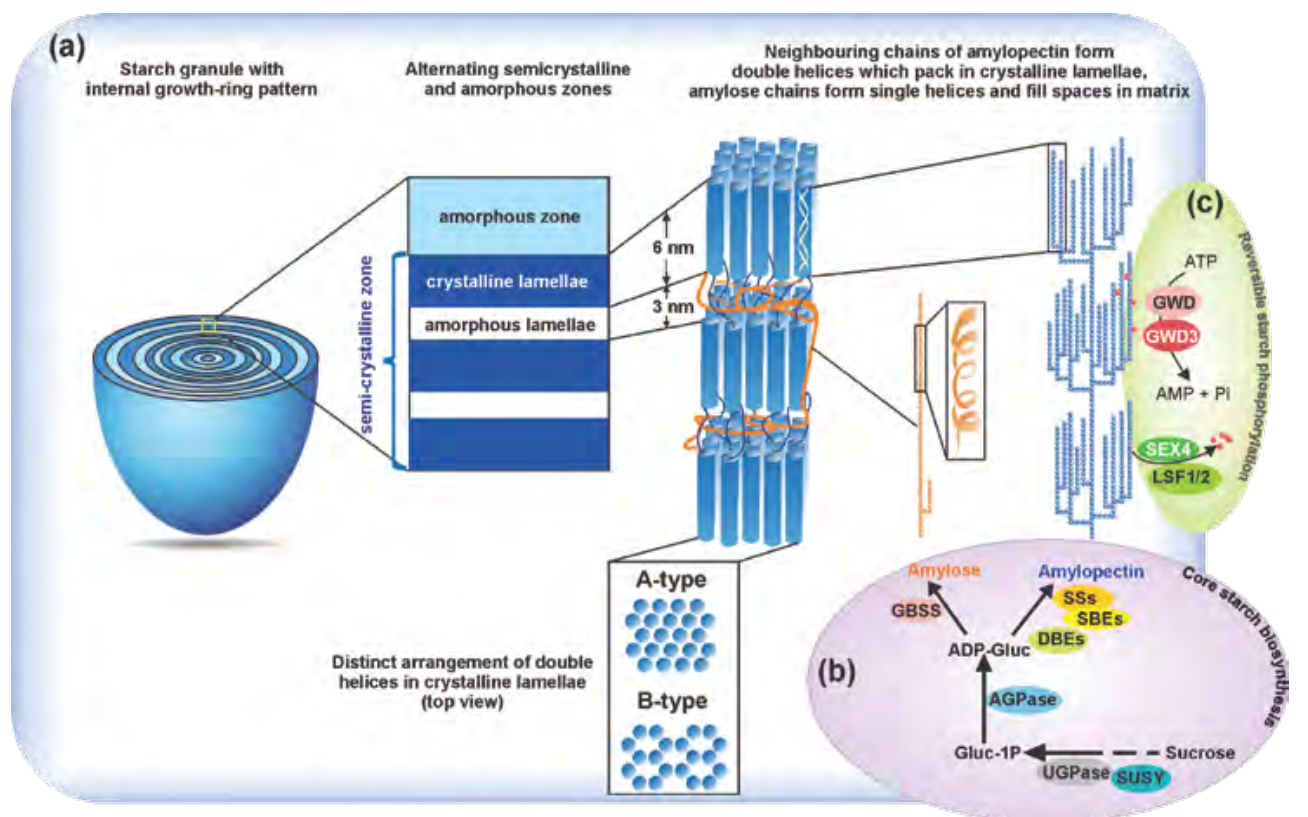

FIGURE 2. Structure and synthesis of a starch granule. (a) Starch granule architecture and molecular organization. The internal structure of a starch granule displays "growth ring" patterns. Each growth ring is comprised of a semi-crystalline and amorphous zone. Semi-crystalline zone contains alternating crystalline (containing clusters of amylopectin double helices formed by adjacent chains) and amorphous lamellae (containing branch points and most of amylose molecular) with a periodicity of $9 \mathrm{~nm}$. The distinct arrangement of double helices result in two types of polymorphs, designated A- and B-type. A-type polymorphs have high density and are typical for cereal starches, while B-type polymorphs are less dense and present in e.g. tuber starches. (b) Core pathway of starch synthesis. Sucrose is converted into glucose-1-phosphate (Gluc-1P) via a series of enzymes, including sucrose synthase (SUSY) and UDP-glucose pyrophosphorylase (UGPase). ADPglucose (ADP-Gluc) is synthesized via ADP-glucose pyrophosphorylase (AGPase) and used as substrate to synthesize amylose and amylopectin. Amylose is synthesized by granule-bound starch synthase (GBSS), while amylopectin is synthesized through a complex orchestration of soluble synthases (SSs), starch branching enzymes (SBEs) and starch debranching enzymes (DBEs). (c) Reversible phosphorylation of starch. Phosphate esters are added to amylopectin chains by glucan, water dikinase (GWD1) and phosphoglucan, water dikinase (GWD3/PWD) and can be released by starch phosphatases (SEX4, LSF1 and LSF2). The red circles represent phosphate esters, while circles with orange and blue colour represent individual glucosyl residues in the amylose and amylopectin chain. The schematic graph was inspired by Pfister and Zeeman (2016).

\section{Genetic modification of starch structure}

Starch structure, such as the branch chain length, strongly affects the gelatinization, retrogradation (Miles et al 1985; Gudmundsson and Eliasson 1990; Kalichevsky et al 1990; Jane et al 1992; Shi and Seib 1992, 1995; Yuan et al 1993; Lu et al 1997), and pasting properties of starch (Jane et al. 1992, Jane and Chen 1992, Wang et al. 1993). Therefore, modification of starch structure is an appealing target for starch bioengineering. 
As described above, starch structure to a large extent is delineated by the structure of amylopectin, as the double helices of amylopectin are the building blocks for the framework of the crystalline lamellae. It has been demonstrated that amylopectin structure is a result of the orchestration of three major classes of enzymes, including starch synthases (SSs), starch branching enzymes (SBEs) and starch debranching enzymes (DBEs). In brief, SSs and SBEs work concurrently and collaboratively to elongate chains and introduce branch points. Interestingly, DBEs are important determinants of the structure by removing the wrongly-positioned branch points that interfere with double helix formation and prevent or delay the crystallization of starch granules (Ball et al., 1996; Myers et al., 2000; Streb et al., 2008). Despite the progress in identifying the enzymes of amylopectin synthesis, the mechanisms underlying starch structure and granule assembly is not fully understood. For instance, how these enzymes are cooperated to give rise to an optimal structure of amylopectin, and how DBEs can discern clearly wrong chains from right ones. More studies are needed to answer these questions.

The complexity of amylopectin synthesis makes the structure difficult to be effectively modified. Continuous efforts have been made in the past decades; however, modifying starch structure is still a challenging task, which has not been achieved effectively in planta so far (Ball and Morell, 2003; Blauth et al., 2002; Jobling, 2004; Safford et al., 1998b; Satoh et al., 2003). One of a few successful examples is simultaneous downregulation of starch synthases in potato. This resulted in an enrichment of short chains in starch granules and thus an alteration in starch gelatinization temperatures and viscosity (Edwards et al., 1999). In that case, instead of manipulating multiple endogenous genes, expression of a heterologous gene that has potential to impart novel chains to starch structure might be a more efficient approach. The latter was therefore applied to the studies presented in this thesis.

\section{Reversible starch phosphorylation and genetic modification of it}

\section{Reversible starch phosphorylation, a new piece in the puzzle of starch biosynthesis}

In the past decade, a lot of efforts have been made to have a better understanding of starch metabolism by studying both storage and transitory starch. Many fundamental questions regarding starch biosynthesis have been addressed using Arabidopsis thaliana instead of starch crops. When comparing the transitory leaf starch system and tuber and seed endosperm systems, high similarity of enzyme functions and biosynthetic-pathways has been found (Santelia and Zeeman, 2011). It is generally accepted that starch biosynthesis works as a refined balance wheel between a continuous synthesis and degradation. It is derived from the complex orchestration of a range of synthesizing and degrading enzymes, including sucrose synthase (SUS), ADP-glucose pyrophosphorylase (AGPase), 
starch synthases (GBSS and SSs), starch branching enzymes (SBEs), starch debranching enzymes (DBEs), a/ $\beta$-amylases (AMY/BAM) and starch phosphorylating enzymes (GWDs) (Xu et al., 2014; Zeeman et al., 2010). These enzymes play a role in one or more of the three major steps (i) the conversion of sucrose to glucose-6-phosphate, (ii) the synthesis of substrate adenosine diphosphate glucose (ADP-Glc) and (iii) the synthesis of starch from ADP-Glc (Fig. 2b, more details in Chapter 2).

Over the recent years, the models of starch biosynthesis have evolved to include the phosphorylation of starch (Fig. 2c). It was shown that phosphate esters are present in starches from various organisms and the level varies considerably among botanical sources. For instance, the concentration of phosphate esters is extremely high in tuberous starch $(\sim 30 \mathrm{nmol} / \mathrm{mg})$ in contrast to that in cereal starch $(\leq 1 \mathrm{nmol} / \mathrm{mg})$. Earlier studies have confirmed that phosphate esters are exclusively bound to amylopectin chains as monoesters mainly at the C-6 ( 70\%) and C-3 (30\%) positions of the glucose units (BaySmidt et al., 1994; Blennow, 2015; Hizukuri et al., 1970; Takeda and Hizukuri, 1982).

Two enzymes have been identified to be responsible for this process: glucan, water dikinase (GWD1) and phosphoglucan, water dikinase (GWD3). Both enzymes catalyse the transfer of the $\beta$-phosphate of ATP to a glucosyl residue of amylopectin (Baunsgaard et al., 2005; Kotting et al., 2005; Ritte et al., 2002). GWD1 phosphorylates glucose residues exclusively at the C-6 position (Ritte et al., 2006). Suppression of this gene in potato plants leads to a starch excess phenotype in leaves and inhibition of cold sweetening in tubers (Lorberth et al., 1998). A similar phenotype was also observed in the leaves of arabidopsis deficient in GWD1 (Yu et al., 2001a). By contrast, GWD3 phosphorylates glucose residues at the $\mathrm{C}-3$ position with substrate specificity requirements for pre-phosphorylation of starch (Baunsgaard et al., 2005; Kotting et al., 2005). Arabidopsis GWD3 mutants show a mild starch excess phenotype, which indicates the essential role in normal starch degradation (Baunsgaard et al., 2005; Kotting et al., 2005). In addition, arabidopsis also contains a third glucan, water dikinase (GWD2), which is an active cytosolic isoform of GWD1 (Glaring et al., 2007). It is not required for starch degradation and the function of this enzyme has not been elucidated (Glaring et al., 2007). Further studies suggested that the phosphate esters lead to local order disturbance and subsequently facilitate the access and action of degrading enzymes (Blennow and Engelsen, 2010; Blennow et al., 2002; Hansen et al., 2008). In contrast to the clear relationship between starch phosphorylation and starch degradation, the effect of starch phosphorylation on starch synthesis needs to be explored further. Only a few studies, to date, have suggested that starch phosphorylation somewhat influences starch synthesis (Carciofi et al., 2011; Skeffington et al., 2014); however, no direct evidence so far can demonstrate role of phosphorylation in starch synthesis. 
On the other hand, phosphatases have been shown to be involved in removing the phosphate esters added by GWDs for complete starch degradation. Similar to the GWD1 and GWD3 mutants, starch degradation in arabidopsis mutants lacking the phosphatases is severely compromised during the night (Zeeman et al., 1998), leading to a progressive accumulation of starch (Zeeman and Rees, 1999). This is because $\beta$-amylases cannot access glucan chains carrying phosphate esters to further release maltose, thus hindering the full degradation of the glucan chains (Kotting et al., 2009). Several phosphatases have been identified in arabidopsis. One phosphatase named Starch Excess 4 (SEX4) has been demonstrated to dephosphorylate phosphate esters at both C-3 and C- 6 positions, whereas another phosphatase termed Like Sex Four 2 (LSF2) is specific for the removal of C-3 phosphate esters. An additional homologue of SEX4, called Like Sex Four 1 (LSF1), is also required for normal starch breakdown although /sf1 mutants show a less pronounced phenotype than the sex4 mutants.

In fact, the first glucan phosphatase discovered was in human, named laforin. It was identified as a key gene responsible for the fatal neurodegenerative epilepsy known as Lafora disease (Emanuelle et al., 2016; Worby et al., 2006). Interestingly, lafroin has structural and functional similarities to SEX4 (Gentry et al., 2007) and is able to remove phosphate esters from amylopectin in vitro (Worby et al., 2006).

Taken together, it is evident that reversible phosphorylation is indispensable for starch metabolism; however, many of the discoveries have been obtained from studies of transitory starch in leaves and little is known about reversible starch phosphorylation in storage organs. In this light it is interesting and essential to explore the role of starch dikinases and phosphatases in potato, hence advancing our understanding of the role of reversible starch phosphorylation in storage starch metabolism.

\section{Genetic modification of phosphate content}

Potato starch has the highest level of phosphate content among the main starches used for commercial purposes and this imparts a remarkable value to its end-uses. For industrial uses, phosphate esters contribute extraordinary water-binding capacity to starch (Muhrbeck and Eliasson 1987; Wiesenborn et al. 1994; Vikso-Nielsen et al. 2001; Jobling 2004). Starches with high phosphate content produce clear and viscous pastes and have improved freeze-thaw stability. They are often used in refrigerated and frozen foods as emulsion stabilizers (Singh et al. 2007), as well as in the paper-making industry as surface coatings (Blennow 2015). Therefore, engineering starch with altered phosphate content carries great potential to modify starch properties.

Currently, genetic modification of starch phosphate content in crop plants is focussed on modulating the expression of GWD1. For instance, an increased starch phosphate content via overexpressing of GWD1 has been achieved in many crops, including rice (Frohberg et 
al., 2008), maize (LanahanMichael and Ship, 2005), wheat (Schewe et al., 2002), and barley (Carciofi et al., 2011). Surprisingly, GWD1 has not yet been overexpressed in potato to obtain starches with high phosphate content, although the role of GWD1 was first discovered in the potato. On the other hand, it has been demonstrated that modulating other enzymes involved in starch biosynthesis may have effects on starch phosphate content. For example, suppression of SBEs in potato resulted in threefold increase in phosphate content (Schwall et al., 2000). Additionally, in our opinion, other starch phosphorylating enzymes (GWD2 and GWD3) and phosphatases are potential tools to modulate phosphate content in planta due to their ability to add or remove starch phosphate esters. If so, these enzymes will become additional assets in the toolbox of genetic modification of starch phosphate content, and this is worth endeavors in this field.

\section{Objectives and outline of this thesis}

Genetic modification of starch has a great potential to substantially improve starch functionalities and reduce or eliminate the use of costly and environmentally unfriendly post-harvest modification processes. This offers a great opportunity for building up an economical and sustainable starch industry and thus a robust bio-based circular economy to overcome challenges related to a growing population and climate change. Therefore, the main purpose of this study is to produce potato starches with novel properties in planta, meanwhile to gain a better understanding of storage starch biosynthesis. To this end, a number of enzymes derived from different sources were introduced into potato tubers to modify phosphate content and starch structure. Some of these enzymes were fused to SBD and targeted to granules during starch biosynthesis to enhance the impact of the enzymes on starch characteristics.

Chapter 2 reviews the state-of-art of biotechnology for starch modification. This chapter focuses on the characterization of a wide range of modified starches that have been generated using biotechnological approaches and the implications of these genetic modifications on the morphology of the starch granules and subsequent physico-chemical properties. Additionally, an overview of the functionality of key enzymes involved in starch biosynthesis and the bottlenecks for the production of genetically modified starch have been provided and discussed.

In Chapter 3, a human phosphatase enzyme named laforin and modifications of it were introduced into potato plants to modify starch phosphate content. The effects of the (engineered) laforin on phosphate content, composition and properties of potato starch were investigated. The correlations between starch structural characteristics and properties were explored. Furthermore, quantitative RT-PCR analysis was used to investigate whether the expression of genes involved in starch biosynthesis was affected in the transgenic plants. 
In Chapter 4, a glucan, water dikinase (GWD1) was expressed in tubers of two different potato genetic backgrounds: an amylose-containing line and an amylose-free mutant. Through exhaustive characterization of modified starches, we have investigated in detail the effects of starch phosphate content on starch morphology, fine structure and properties. The possible role of phosphate esters in the formation of starch granules and effects of phosphate esters on starch properties are discussed with respect to the potato background. The effect on starch metabolism-related genes was investigated by RNA-sequencing (RNA-seq) and quantitative RT-PCR (qRT-PCR) analysis in an attempt to identify the role of starch phosphorylation on starch biosynthesis.

In Chapter 5, two starch dikinases from Arabidopsis thaliana, glucan water dikinase 2 and 3 (AtGWD2 and AtGWD3), were heterologously expressed in potato tubers (Kardal and amf genetic background) to increase the phosphate content of potato starch and to explore the effect of starch phosphorylation on storage starch metabolism. Starch structure, composition, granule size and morphology, as well as properties are well characterized by a comprehensive and systematic analysis. The feasibility of enhancing phosphate content in already naturally highly phosphorylated potato starch is discussed.

Chapter 6 describes attempts to introduce novel chains into starch by expressing an (engineered) 4, 6-a-glucanotransferase (GTFB) from Lactobacillus reuteri 121 in potato tubers. In this chapter, we have investigated whether this target gene is able to change the branching pattern of glucan chains in and thus the structure of starch in the granules. The alterations in granule morphology and size, starch composition and properties are presented. Transcriptome analysis was used to investigate the changes in expression of starch synthesizing and degrading genes.

Finally, the most relevant results obtained from all above chapters are summarized and integrated in Chapter 7.I discuss the implications of the findings and future prospects of modification of starch in planta. 


\title{
Chapter 2
}

\section{Starch modification by biotechnology: state of art and perspectives}

\author{
Xuan $\mathrm{Xu}^{1,2}$, \\ Richard G.F. Visser ${ }^{1}$, \\ Luisa M. Trindade ${ }^{1}$
}

${ }^{1}$ Wageningen UR - Plant Breeding, Wageningen University and Research, P.O. Box 386. 6700 AJ Wageningen, The Netherlands.

${ }^{2}$ National Centre for Vegetable Improvement (Central China), Key Laboratory of Horticultural Plant Biology, Ministry of Education, Huazhong Agricultural University, Wuhan, 430070, China.

Published in: Starch Polymers: From Genetic Engineering to Green Applications pp. 79-104 (2014). 


\begin{abstract}
Starch, the most important storage carbohydrate, can be modified into diverse products widely used in food and non-food industry. With the understanding of the starch biosynthesis and degradation pathways, starches with novel functionalities have been made in planta. These might be used in future due to their perceived broadening of the range of starch applications, the expected ease of the processing, and absence of environmental pollution. This chapter focuses on characterization of modified starches that have been generated using biotechnological approaches around three aspects. 1. Synthesis of novel starch by manipulating endogenous genes involved in starch biosynthesis and degradation pathways; 2 . The expression of heterologous genes encoding biosynthetic or modifying enzymes to produce designer starches with novel functionalities; 3. Industrial application of improved starches created by biotechnological modification.
\end{abstract}




\section{Introduction}

Carbohydrates are the most abundant bio-compounds on our planet. More than 100 billion metric tons of $\mathrm{CO}_{2}$ and $\mathrm{H}_{2} \mathrm{O}$ are converted into carbohydrates and other plant compounds by green plants during the process of photosynthesis every year (Nelson and Cox, 2004).

Starch, the major storage carbohydrate, is synthesized by most green plants and can be found in various types of tissues and organs. It is an insoluble glucan composed of two polymers: linear amylose (20-30\%) and branched amylopectin (70-80\%) (Kossmann and Lloyd, 2000). Although both are composed of glucosyl units linked by a-1, 4-bonds and branched in a-1, 6-bonds, the starch granule structure is more complex than expected from its components. The polymeric carbohydrates of the starch are organized in alternating amorphous and semi-crystalline rings (Jenkins et al., 1993b), and the forming of the semicrystalline organization is mainly dependent on amylopectin which has relatively short chains.

In plants, starch is synthesized in plastids and two types of starch can be distinguish, the transitory starch and the storage starch. The transitory starch is synthesized and accumulated in chloroplasts of green leaves during daytime photosynthesis and degraded throughout the night to provide substrates for leaf respiration and for continued sucrose synthesis to supply to sink tissues (Zeeman and Rees, 1999). Mutants of Arabidopsis thaliana unable to synthesize transitory starch grow slower in the day-night regime relatively to the wild type, because they lack carbon supply in the dark (Caspar et al., 1985). Storage starch is synthesized in amyloplasts and typically associated with sink organs, such as stems, seeds, roots and tubers. This type of starch is accumulated during different developmental stages and utilized for various periods of growth, dormancy, germination or other specific processes. Apart from the different locations in the cell, the two types of starch have different characteristics. Storage starch is deposited in larger granules and contains a higher amylose content than that observed in transitory starch (Jobling et al., 2002; Matheson, 1996). Next to these differences within plants, there are also differences between plant species. For instance tuber and root starches have lower protein and lipid contents than cereal starches, which have advantages in many applications. In a word, patterns of starch accumulation, starch granule size, shape and physicochemical properties differ not only between plants of different botanic origins but also within accessions of the same species.

The importance of starch is not limited to the relevance of this polysaccharide in plant survival but, to a large degree, due to the application in food and non-food industry. With the imminent depletion of fossil oil reserves, starch has gained increasingly interest as a sustainable and renewal feedstock for the production of biochemicals, biomaterials and 
biofuels. With the marked development of industrial economy, starch, which is isolated from maize, rice, wheat, cassava, potato, pea, arrowroot (Marantaarundinacea) and sago palm (Metroxylonsagu), has become a cornerstone of a sustainable industry (Zeeman et al., 2010). It has been shown that starch structure highly affects the physicochemical properties, such as gelatinization, texture, moisture retention, viscosity and product homogeneity, which is determinant for its applications.

Native starches have shortcomings that restrict the end uses. Properties such as retrogradation and gelling tendencies of pastes are undesirable in many application fields (Kaur et al., 2012). Starch derivatization comprises the use of chemical, enzymatic and/or physical modifications which require high amounts of energy and chemicals and produce chemical waste. Hence, modification of starch in planta could improve the functional properties with a broad range of specific applications and produce starches with better quality, using less energy and without pollutant chemical waste (Kaur et al., 2012). Up to now, modified starch has been widely used for many industrial applications such as dairy confectionery, meat products, oil drilling, paper and cardboard, adhesives, packing material, pharmaceuticals, textile sand bioplastics (Ellis et al., 1998; Roper, 2002). It is worth noticing that genetic modification of starch in planta has received increased attention, not only due to the enormous potential for tailoring granules with new functionalities, but also to reduce the costs of the post-harvest modifications and usage of other approaches which probably cause damage to the environment (Kok-Jacon et al., 2003)2003. Understanding of the starch biosynthesis and degradation pathways will facilitate the progress of creating designer starches that have novel functionalities by using biotechnology.

This chapter focuses on the characterization of modified starches that have been generated using biotechnological approaches and the implications of these genetic modifications on the morphology of the starch granules and the subsequent starch physical-chemical properties.

\section{Starch biosynthesis and mutants in the starch biosynthetic pathway}

In plant's lifecycle, the biosynthesis of starch works as a refined balance wheel between a continuous efficient assembly and degradation of glucan chains. The synthesis of transitory and storage starch in the chloroplast and amyloplast involves several common enzymes as well as specific isoforms that are only involved in the synthesis of one of these two types of starch.

Current knowledge about starch biosynthesis in plants show that the ADP-glucose pyrophosphorylase (AGPase, E.C.2.7.7.27), starch synthases (SS), granule-bound starch synthase (GBSSI), starch branching enzymes (SBE) and possibly the starch debranching 
enzymes (DBE), play a role in determining the final starch structure (Smith et al., 1997). These enzymes are present in one or more of the three major steps (i) activation of glucose residues, (ii) chain elongation and (iii) synthesis of side chains (Fig.1).

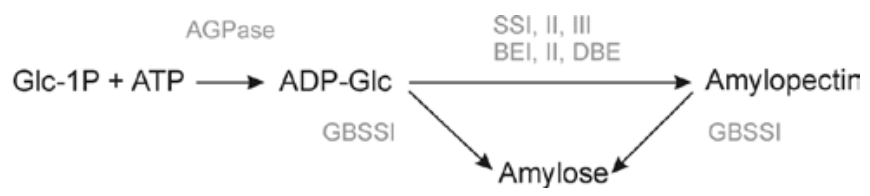

FIGURE 1. Amylose and amylopectin biosynthesis. ADP-Glucose (ADP-GIc), the donor substrate for both amylose and amylopectin, is synthesized by the ADP-Glc pyrophosphorylase (AGPase). The combine action of different starch synthases (SSI, SSII and SSIII), branching enzymes (BEI and BEII) and the debranching enzyme (DBE) are necessary for the synthesis of amylopectin. Granule bound starch synthases (GBSSI and GBSSII) use amylopectin as the acceptor substrate to synthesize amylose, which is formed downstream of amylopectin.

\section{Initiation of starch biosynthesis}

The first step in starch biosynthesis, the synthesis of ADP-glucose is catalysed by ADPglucose pyrophosphorylase (AGPase, E.C.2.7.7.27), first found in wheat flour (Espada, 1962). This enzyme converts glucose 1-phosphate (Glc-1-P) into adenosine diphosphoglucose (ADP-Glc, (Preiss et al., 1988), which is the preferred substrate for starch formation. This enzyme also was found in many plant tissues and bacterial extract (Preiss and Sivak, 1998), however, the localization and regulation of ADP-Glc synthesis and import Glc-1-P is highly various between different species and in different organs within a species (Emes and Neuhaus, 1997; Neuhaus and Emes, 2000). As a key factor of this rate-controlling step, AGPase was extensively used, through biotechnology, to control the rate of ADP-Glc formation and, in turn, starch accumulation. The amylose content was severely reduced in transgenic potato which contained a lower expression of AGPase (Lloyd et al., 1999). A mutation at the $r b$ locus from pea (Pisum sativum L.), which reduced the activity of AGPase (Smith et al., 1989), resulted in a decrease of starch in the embryo (Kooistra, 1962). Interestingly, inactivation of AGPase in a Chlamydomonas starch less mutant led to a 10fold increase in TAG that can be used to produce biofuels (Li et al., 2010), but only to a slight decrease in the accumulation of starch (Ball et al., 1991). In addition, a mutant E. coli AGPase gene (glgc16) has been over-expressed in some plants to enhance starch accumulation, such as potato (Stark et al., 1992)m and maize (Wang et al., 2007).

Some observations indicated that conversion of sucrose to starch is ATP dependent. The ATP/ADP transporter (AATP), located on the inner-envelope membrane, imports ATP required for the AGPase reaction to the plastid (Heldt and Pfaff, 1969; Schunemann et al., 1993). Overexpression of a ATP/ADP transporter, an energy supplier for the AGPase reaction within the plastid, leads to an increase of $16-36 \%$ in starch content, in contrast, antisense ATP/ADP transporter tubers exhibited drastically reduced levels of starch 
(Tjaden et al., 1998). In chloroplasts, the catalytic activity of AGPase is allosterically activated by 3-phosphoglycericacid (3-PGA) and inhibited by inorganic phosphate (Pi). The allosteric properties of AGPase are of potential importance in determining its role in controlling the rate of ADP-Glc formation and, in turn, starch biosynthesis (Smith et al., 1997). The small and large subunits composition of AGPases mainly affect the allosteric properties. The small subunit alone can form a catalytically active enzyme and the large subunit is responsible for modulation of the sensitivity of the enzyme to effectors. The sta1 mutant in the unicellular algae Chlamydomonas reinhardtii, containing lesions in the large subunit of AGPase, leads to a 95\% reduction in starch biosynthesis (Ball et al., 1991; VandenKoornhuyse et al., 1996). Maize mutants where the genes encoding the large subunit and small subunit of AGPase have been knocked out, respectively shrunken-2 and brittle-2, demonstrates that in the endosperm the primary flux to starch is via the cytosolic enzyme. In seeds of the shrunken-2 mutant the amount of starch was significantly increase by $15 \%$.

\section{Chain elongation}

Synthesis of starch is catalysed by starch synthases (SS, EC 2.4.1.21) which transfer the glucose residue from ADP-Glc to the non-reducing end of a growing a-1,4 linked glucan. Six isoforms of starch synthases have been characterised by multiple sequences alignment. SSI, SSII, SSIII and SSIV are present in a diverse range of species as soluble forms or as both soluble and minor granule-bound isoforms, whereas granule-bound starch synthase (GBSS), including GBSSI in storage organs and GBSSII in non-storage organs, is involved in amylose biosynthesis. A novel high-amylose starch was discovered in the barley sex6 mutant, which was lacking the activity of SSIla (Moire et al., 2003). Compared to the high-amylose starches produced by down regulation of starch branching enzyme (SBE), the sex6 starch has amylopectin with relatively short branches and gelatinised at lower temperatures. The synthesis of amylose-free starches has focused on engineering GBSS activity in different plant species. Amylose-free starches were reported in mutants of maize (waxy, Nelson and Rines, 1962), , sorghum (waxy, Hsieh, 1988), wheat (waxy, Nakamura et al., 1995), amaranth (waxy, Okuno and Sakaguchi, 1982), pea (lam, Denyer et al., 1995) and unicellular green algae Chlamydomonas reinhardtii (st-2-1 and st-2-2, Delrue et al., 1992). The activity of GBSS is inexistent in these mutants resulting in starches with better properties for industrial applications; however, they show sometimes small yield penalties which can be fixed by selection, irrespectively of the method used to generate the mutants.

Amylose-free starches also have been produced by down-regulation of GBSS in potato (Visser et al., 1991) and sweet potato (Kimura et al., 2001; Noda et al., 2002), and no significant penalties on the starch content were observed. The natural GBSS mutant 
genotype producing amylose-free potato starch (Hovenkamphermelink et al., 1987) has been explored commercially for many years such as the Eliane ${ }^{\mathrm{TM}}$ commercialized by AVEBE (www.avebe.nl). The amylose-free cassava starch derived from a mutant is opening the door to commercial applications in this crop as well (Ceballos et al., 2007). Jobling et al. (2002) observed a novel amylose-free starch with short-chain amylopectin via the simultaneous down-regulation of GBSS, SSII and SSIII.

\section{Formation of branch-points}

The formation of the $a-1,6$ linkages as branching point of the $a-1,4$ glucan chains is of great importance in determining amylopectin structure. Comparing the starch branching enzyme (SBE, EC 2.4.1.18) sequences from a wide range of species revealed two distinct classes of branching enzymes in plants: SBE A and SBE B. The analysis of mutants has provided direct evidence that expression levels of SBE affect the nature of synthesised starch. The mutation in the $r$ locus in wrinkled peas which is associated with complete absence of one isoform of starch-branching enzyme (SBEI) leads to an amylopectin content which is reduced from about $70 \%$ to about $30 \%$ of the total starch content (Bhattacharyya et al., 1990). Antisense of the major isoform (SBE B) in potato reduces starch branching enzyme activity in the tubers almost completely without affecting the amylose content in the starch (Kossmann et al., 1997; Safford et al., 1998a). Schwall et al. (2000) created highamylose potato starch (more than $60 \%$ of the granule) by simultaneously inhibiting two isoforms of starch branching enzyme to below $1 \%$ of the wild-type activities. Some results suggest that these enzymes are redundant. The antibody-mediated inhibition of SBEA activity is more efficient than using antisense technology and produces higher amylose levels (Jobling et al., 2002).

The action of debranching enzyme (DBE; EC 2.4.1.41) activities during biosynthesis is considered to be critical for the accurate assembly of the starch granule. Two types of DBE exist in plants, isoamylase (ISA) and limit-dextrinase (LDA or pullulanase). The former one has a higher affinity for glycogen, while the latter one has a higher affinity for pullulan (Doehlert and Knutson, 1991). Both enzymes are responsible for hydrolysing the a-1.6 branch points that are not correctly positioned in the molecule and for generating a branching structure appropriate for crystallization (Ball and Morell, 2003). Likewise SS and $\mathrm{SBE}$, the role of DBE in the synthesis of amylopectin has been proposed by several studies with DBE-deficient mutants of various plant species, such as rice (Nakamura, 1996), maize (James et al., 1995; Pan and Nelson, 1984), arabidopsis (Zeeman et al., 1998) and Chlamydomonas (Mouille et al., 1996). The results showed that wild type amylopectin was replaced by glycogen-like a-glucanphyto glycogen and both morphological and physicochemical properties of starch were altered to a certain degree. 


\section{Starch degradation and mutants in the starch breakdown}

Starch degradation has been better studied in transitory starch in leaves, where starch is broken down during the night, than in storage starch where it is degraded over a relatively long time. Although starch degradation has been widely studied in cereal endosperm (Fincher, 1989; Ritchie et al., 2000), where starch is broken down over several days after seed germination, the mechanism and regulation of this process in other starchcontaining organs such as roots, tubers and non-cereal seeds are more fragmentary. This is most probably due to the fact that starch degradation pathways differ depending on the plant organ, and different pathways may operate within the same organ (Zeeman et al., 2010). At least for arabidopsis, the important genes have been identified and the major steps in the pathway are known. The initiation of starch degradation is the reversible phosphorylation of glucans at the surface of the starch granule by a class of enzymes called glucan water dekinases (GWD, Ritte et al., 2002). Subsequently, hydrolases access to the glucan chains and starch is hydrolysed to maltose and glucose and both can be exported from the chloroplast and metabolized in the cytosol.

\section{The enzymatic breakdown of starch granules}

The initial event, seemingly required for starch degradation, must be catalysed by an enzyme capable of metabolizing polymers on the surface of semicrystalline granules to release the soluble glucans. Three most likely candidates, a-amylase (AMY), $\beta$-amylase (BAM) and debranching enzyme (DBE), are capable of attacking the starch granule surface. Starch degradation occurs in germinating cereal endosperm, and is thought to be initiated by AMY based on the characteristic visible pitting of the starch surface. It seems that AMY is in charge of attacking the insoluble granule, hydrolysing a-1.4 linkages within polymers exposed on the surface or in channels within granules to release both linear and branched glucans for further degradation.

However, surprisingly, the studies in arabidopsis suggested that AMY is much less significant in starch breakdown. Three proteins predicted to be a-amylases (AMY1, 2 and 3) are encoded in the arabidopsis genome. T-DNA insertion mutants lacking these isoforms respectively have normal rates of starch degradation in leaves at night, and starch metabolism also appears normal in amy1/amy2/amy3 triple mutants ( $\mathrm{Yu}$ et al., 2005). These results suggest that AMY is not required for starch degradation in arabidopsis, whereas the studies described recently suggest that in some circumstances, AMY 3 does participate in degradation of starch granule in arabidopsis (Zeeman et al., 2007a). It was reasonable to assume that there are other endoamylases involved in starch degradation that compensate for the deficiency of AMY3 both in arabidopsis and potato (Zeeman et al., 2007b). The loss of a specific isoform of $\beta$-amylase (BAM3 in arabidopsis; PCT-BMY1 
in potato) and debranching enzyme isoamylase (ISA3 in arabidopsis and potato) lead to the decreased rates of starch breakdown and excess accumulation of starch, indicated that these two enzymes affected the degradation of starch granules (Delatte et al., 2006; Kaplan and Guy, 2005; Scheidig et al., 2002; Wattebled et al., 2005). In addition, suppressed expression or overexpression of AMY in rice caused the correspondent changes of starch accumulation in leaves (Asatsuma et al., 2005).

\section{The importance of glucan phosphorylation}

The glucan water dikinase (GWD) plays an important role in starch degradation, and was first discovered in potato tubers by Lorberth et al. (1998). This enzyme transfers the $\beta$-phosphate group of ATP to a small proportion of the glucose residues of amylopectin chains, particularly at the 6-position (Mikkelsen et al., 2004; Ritte et al., 2002). The formed phosphate groups could affect the structure of the starch granule and thus the starch surface become more vulnerable to facilitate starch degradation (Blennow et al., 2000 b). By antisense technology in potato it was determined that down-regulation of GWD leads to a reduction in the phosphate content of the starch, and thereby impairs starch degradation in leaves and reduces cold sweetening in tubers (Lorberth et al., 1998). This result is similar with the data obtained from studies of arabidopsis mutants of deficient GWD protein. Even the mature leaves of these mutants increase amounts of starch up to seven times greater than those in wild-type leaves (Caspar et al., 1991; Yu et al., 2001b; Zeeman and Rees, 1999). The picture of starch degradation has evolved to include the second GWD-like enzyme, phosphoglucan water dikinase (PWD, Baunsgaard et al., 2005; Kotting et al., 2005). An arabidopsis mutant with down-regulated expression of this gene has increased amounts of starch in leaf (Baunsgaard et al., 2005). PWD will act on phosphorylated amylopectin rather than unphosphorylated glucans, therefore it was presumed that the activity of PWD requires the presence of active GWD. With the discovery of GWD and PWD, it is now possible to create the starch of high phosphate content in plants and particularly in cereals which are extremely low in phosphate content. Overexpression of GWD has been achieved in maize (LanahanMichael and Ship, 2005), rice (Frohberg, 2008), wheat (Schewe et al., 2002) and barley (Carciofi et al., 2011) resulting in increased starch phophate content.

\section{Expression of heterologous enzymes in plants}

Progress in understanding the high value of modified starches has encouraged researchers to produce modified starches using different strategies. Expression of heterologous genes from other organisms, which encode biosynthetic or modifying enzymes, have the potential to be one of the most economical systems for large-scale production of starches 
with novel properties. Bacterial genes provide an ideal source of variety because they may have properties that are slightly different from their plant counterparts and thus create different or novel phenotypes.

Several starch modifying bacterial proteins have been introduced in different crops, including potato, maize and cassava. These included: a starch binding domain (SBD) of Bacillus circulans cyclodextrin glycosyltransferase, an E. coli AGPase (GLGC16), an E. coli glycogen synthase (GLGA), an E. coli branching enzyme (GLGB), an E. coli maltose acetyltransferase (MAT), a Neisseria polysaccharide amylosucrase, a full-length (GTFI) and a truncated mutansucrase (GTFICAT) from Streptococcus downei, a L. mesenteroides dextransucrase (DSRS), and a L. mesenteroides alternansucrase (ASR).

A starch binding domain (SBD) of cyclodextrin glycosyltransferase from Bacillus circulans was expressed in potato tubers. SBDs accumulated in the starch granule during the biosynthesis process. The amalgamated starch granules consisted of many smaller granules have been achieved by expression of multiple appended SBDs (SBD2-SBD5) in amf mutant potato plants (Ji et al., 2004; Nazarian-Firouzabadi et al., 2012).

A mutant variant of the AGPase gene from E. coli (GLGC16) was overexpressed in various plant species, such as cassava (Ihemere et al., 2006), maize (Wang et al., 2007) and potato (Sweetlove et al., 1996), and the starch content is slightly increased. Surprisingly, Stark et al. (1992) targeted this gene to plastids in transgenic potatoes resulting in significantly increased starch accumulation, and in some lines there was almost a $60 \%$ increase in starch content relative to control tubers.

The potato tuber amyloplasts expressing the $E$. coli glycogen synthase gene (GLGA) were found to have a lowered specific gravity, about $50 \%$ reduction in the percentage of starch, and an increased branching degree of amylopectin (Shewmaker et al., 1994). For producing starches with higher branching degree of amylopectin and novel properties, the E. coli branching enzyme (GLGB) was introduced in amylose containing background (Krohn et al., 1994) and amylose-free potato mutant (Kortstee et al., 1996). In the former a slightly higher amylopectin percentage and a small difference in granule surface morphology was observed, while the branching degree of amylopectin was $25 \%$ higher in the latter as compared to the control.

Efforts have been made to introduce the maltose acetyltransferase gene (MAT) from $E$. coli into wild type and mutant amylose-free (amf) potato plants in order to increase the acetylation of starch. A low concentration of acetyl groups was found in the starch granules of transgenic plants and also the starch granule morphology was altered (Nazarian Firouzabadi et al., 2007).

In order to alter the ratio of amylose and amylopectin the amylosucrase gene from Neisseria polysaccharide fused to a starch binding domain was introduced in potato which lead to a 
number of changes in the morphology of the starch granule and biochemical properties, but not to a change in the ratio of amylose and amylopectin (Huang et al., 2014).

The production of bacterial extracellular polysaccharides in planta is another great way to generate material with unique nutritional or commercial added-values (Moire et al., 2003). Glucansucrases, produced by soil bacteria, Streptococcus species form the oral flora and lactic bacteria Lactococci, catalyse synthesis of different a-glucans, such as mutan, dextran, alternan and reuteran (Mooser, 1992; Sidebotham, 1974), production of these polysaccharides in the amyloplast could alter the properties of starch and be beneficial to replace post-harvest chemical starch modifications by a more environmental-friendly bioprocessing.

\section{a. Mutan}

Mutansucrase (GTFI, E.C. 2.4.1.5) synthesizes the most adhesive and water-insoluble glucan which is known as mutan (Hamada and Slade, 1980). In terms of basic research, two classes of chemical linkages were catalysed by GTFI. One class is a-1,3 linkages (up to $90 \%$ ) that is responsible for the backbone of mutan, and the other is a-1,6 linkages which assemble single unit glucosyl residues to the backbone (Monchois et al., 1999). Expression of the GTFI gene, which was derived from oral cariogenic Streptococcus downei Mfe28 bacteria (Ferretti et al., 1987), results in the accumulation of mutan polymers in the presence of sucrose. To develop the industrial applications of mutan polymers, different studies with genetically engineered GTFI have been carried out.

One example is the investigation of the production of mutan polymers in amyloplasts of an amylose-containing potato by expressing a full-length (GTFI) and a glucan-binding domain-truncated gene (GTFICAT). By comparing transformants, GTFICAT plants showed a severely altered tuber phenotype and starch granule morphology in addition to starch structural changes (Fig. $2 \mathrm{E}$ and F). Next to that, the starch content was decreased in GTFICAT transformants, possibly because expression of this gene interferes with starch biosynthesis by down-regulating the AGPase gene (Kok-Jacon et al., 2005b). Further, in order to carry the GTFICAT enzyme close to or rather inside the growing starch granule, GTFICAT was fused to the $\mathrm{N}$ - or C-terminus of a starch-binding domain (SBD) and introduced in amylose-containing and amylose-free potatoes respectively (Firouzabadi et al., 2007). In these transgenic plants mutan polymers were incorporated in the starch granule successfully, and resulted in alterations of starch properties such as a higher melting temperature, granules with porous and spongy surfaces, and a more pronounced retrogradation behaviour. However these alterations, together with mutan polymers seemed to be less strikingly than those from transformants with GTFICAT alone, implying that the appended SBD might interfere with the activity of GTFICAT (Firouzabadi et al., 2007). 

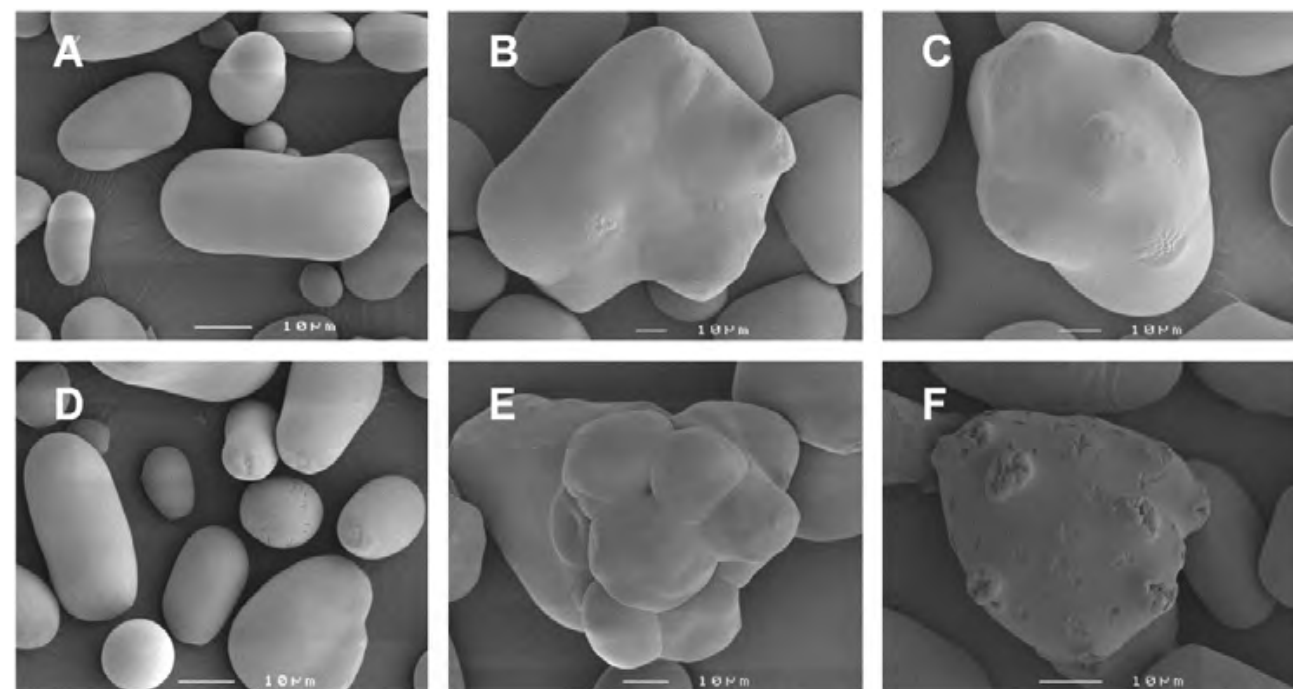

FIGURE 2. Scanning electron microscopy (SEM) analysis of starch granules from untransformed potato plants, cV Kardal (A) and amylose free (amf, panel D), and transformed with the dextransucrase encoding gene $d s r s(B)$, with the alternansucrasse encoding gene $\operatorname{asr}(\mathrm{C})$, with the full length mutansucrase gene gtfl (E) and a truncated for of the latter $g t f l-C A T(F)$.

\section{b. Dextran}

Dextran, an extracellular a-glucan containing a high proportion of a-1,6 glucosidic linkages and associated with variable proportions of $a-1,2, a-1,4$, or $a-1,3$ linkages, is synthesized by the dextransucrase (DSRS, EC 2.4.1.5) of Lactobacillus, Leuconostoc and Streptococcus bacteria in the presence of sucrose (Jeanes et al., 1954; Kralj et al., 2004; Monchois et al., 1996; Simpson et al., 1995). One notable difference between Leuconostoc and Streptococcus bacteria is that in the former the formation of dextransucrases, is induced and in Streptococcus species they are constitutively synthesized (Robyt, 1995a). The formation of dextran was observed as the result of bacterial transformation of sucrose solutions into viscous solutions, gels, and flocculent precipitates (Robyt, 1995b). L. mesenteroides B-512F formed only one dextran containing 95\% a-1,6 linkages and 5\% $\mathrm{a}-1,3$ branch linkages, which is most widely studied and commercially produced (Bjorn and Johan, 1948). Kok-Jacon et al. (2005c) introduced this gene both into an amylosecontaining potato cultivar and an amylose-free (amf) potato mutant. In tuber juices, dextrans were detected in both the different genetic background series, and the dextran concentration was two times higher in amylose-containing than in the amf transgenic lines. In addition, the results revealed that despite altered granule morphology (Fig. 2B), the accumulation of dextran did not interfere with the physicochemical properties and starch content. This strategy could open a new avenue for commercialization of dextran production in plant systems. 


\section{c. Alternan}

Alternan is a branched polymer with a unique backbone structure of alternating $a-1,3$ and a-1,6-D-glucosidic linkages, present for $46 \%$ and 54\%, respectively (Côté and Robyt, 1982; Seymour et al., 1979; Seymour and Knapp, 1980). It is branched by 3,6-disubstituted D-glucosyl residues, with approximately 7-11\% branching (Seymour et al., 1977). Therefore, alternan possesses the properties of high solubility and low viscosity as well as resistance to microbial and mammalian enzymes, which is suitable for the creation of material for functional foods (Cote, 1992; Cote, 2002). Leathers et al. (2003); Leathers et al. (2002) isolated strains of Penicillium sp. that modify native, high molecular weight alternan in a novel bioconversion process to a lower molecular weight form with solution viscosity properties similar to those of commercial arabic gum. Alternan synthesized by L. mesenteroides NRRL B-1501 is catalysed by the alternansucrase (ASR, EC 2.4.1.140), which converts sucrose to alternan and fructose, also has been cloned and sequenced (Arguello-Morales et al., 2000). Furthermore, ASR was transferred into potato tubers and alterations in starch properties and morphology were analysed (Kok-Jacon et al., 2007). The low accumulation of alternan (1.2 $\left.\mathrm{mg} \mathrm{g}^{-1} \mathrm{FW}\right)$ resulted in a slight alteration of starch morphology (Fig. 2C). Although, some of the key genes involved in starch biosynthesis seemed to be down-regulated, particularly the AGPase and GBSSI genes, the physicochemical properties of starch remained unchanged (Kok-Jacon et al., 2007).

\section{Industrial application of improved starches}

\section{Approaches for biotechnological modification of starch}

Along with the better understanding of starch structure and enzymes involved in starch biosynthesis, many of the genes that encode these enzymes have been cloned and transformed into plants using Agrobacterium tumefaciens to modify the starch metabolism. Transgenic plants have been generated by down regulation (antisense or co-suppression approaches) or overexpression of endogenous gene or expression of heterologous genes, where starch properties and morphology have been altered. The possibility to produce tailor-made starches in planta, will broaden the functionality of starches in industrial applications (Zeeman et al., 2010). These in planta modified starches, such as the amf starch, are often of better quality relatively to the chemically derivative and it precludes the use of hazardous chemicals and leads to energy savings in the production process (of up to $60 \%$ for e.g. synthetic polymer replacers).

Ji et al. (2003) developed the so-called Starch Binding Domain (SBD) technology to target effector proteins to starch granules during the biosynthesis process. Almost 10\% of all microbial hydrolytic enzymes can bind to substrate mediated by a distinct domain 
referred to as carbohydrate-binding modules (CBMs) (Janecek et al., 2003; Machovic et al., 2005). SBDs from CBM family 20 have the affinity to bind to starch granules and enzymes can acquire affinity to starch granules by fusing to SBDs (Ohdan et al., 2000). By using the SBD technology to generate potato starches with new or improved properties, some heterologous genes, including an E. coli maltose acetyltransferase (MAT) (Nazarian Firouzabadi et al., 2007), a truncated mutansucrase (GTFICAT) (Kok-Jacon et al., 2005a), were successfully incorporated into starch granules and resulted in morphologically altered starch granules.

\section{Application of improved starches}

\section{a. Waxy starches}

Waxy or amf (amylose-free) starch is a new species of starch composed only of amylopectin molecules. Within the last decade there has been intense interest among researchers to create waxy starch in various species by mutating the $w x$ locus that encodes the GBSS protein. Waxy maize containing $100 \%$ amylopectin, whereas normal maize starch contains $75 \%$ amylopectin and 25\% amylose, was found in China in 1909. This mutant starch is mainly used due to its improved of uniformity, stability, and texture not only in food products but also in the adhesive industry due to its pasting characteristics. For instance, Waxy wheat is more suitable for different food applications. Pastas made with durum wheat containing lower amylose content are of better quality (Kim et al., 2003; Sharma et al., 2002).

The potato amf starch produces a clearer film and a stickier paste after gelatinization, and the resulting gel retrogradates less compared to regular potato starch (Visser et al., 1997). Since 2005 the first natural potato variety ELIANETM, obtained through traditional well accepted breeding techniques, is being cultivated and marketed by the starch company AVEBE. Recently, the European Food Safety Authority (EFSA) allowed the company BASF to commercialize potato variety Amflora ${ }^{\mathrm{TM}}$ within the EU. Although it was produced using genetic modification, Amflora ${ }^{\mathrm{TM}}$ is just as safe for humans, animals and the environment as conventional potatoes via the evidence of extremely rigorous studies (Schmidt, 2009). These starches have much better performance in different industrial processes including the manufacturing of paper, adhesive and textiles. In food industry, another much desired property is freeze-thaw stability of gelatinized starch. Normally the use of unmodified starches in frozen foods is achieved by means of chemical modification to prevent the retrogradation of glucan chains. Jobling et al. (2002) created a freeze-thaw-stable potato starch by simultaneous down-regulation of three starch synthase gene (GBSS, SSII and SSIII) in planta. From an environmental point of view and a consumer perspective, this starch may have a great potential for application in the food industry. 


\section{b. High amylose}

High-amylose starches have the ability to form strong gels and films. These particular properties are especially exploited in various industries, such as textiles, candies and adhesives. For example, the high gelling ability of these starches is exploited in jellygum candies (Mason, 2009). Moreover, high-amylose starches are used in extruded and fried snack products to obtain crisps, because they can expand to tender structure when heated or dried (Jobling, 2004). The first high amylose maize hybrid was developed in the 1950s with the discovery of the ae (SBE, 'amylose extender') gene by the Bear Hybrid Corn Company. Amylomaize is the generic name for maize that has amylose content higher than $50 \%$ and is grown exclusively for wet-milling. Likewise, the high-amylose phenotype in cereals or potato is caused by the loss or down-regulation of the corresponding ae gene respectively (Jobling, 2004; Jobling et al., 1999).

Unlike the normal starch, high-amylose starch escapes digestion in the small intestine of healthy individuals and reaches the large intestine where it is fermented by the gut bacteria. The fermentation of starch in the large intestine has health benefits by promoting a better colonic function. This starch, known as resistant starch (RS), is considered the third type of dietary fibre and well suited for food applications (Bird et al., 2000). In Australia, foods enriched in RS as a high amylose maize starch have gained consumer acceptance. A company, Ascentia Pty Ltd, has been established to develop a RS barley cultivar (Hordeum vulgare var. himalaya 292) which was proven to have potential health benefits through reduction of plasma cholesterol and production of increased large bowel short chain fatty acids. The starch of this cultivar is a promising vehicle to deliver health benefits to consumers (Topping et al., 2003). A high-amylose transgenic rice line modified by downregulation of two SBEs in transgenic rice endosperm resulted in high content of resistant starch with high potentialities in food and non-food industry (Wei et al., 2010). Highamylose wheat generated by RNA interference also has a potential to deliver significant health benefits to consumers given its RS content (Regina et al., 2006).

\section{c. Phosphate}

Native starches contain small amounts of covalently bound phosphate. Potato starch shows a naturally high degree of phosphorylation compared to starches from other crops (Hizukuri et al., 1970). The presence of phosphate in potato starch results in the stablepaste properties and transparent gels. Hence, potato starch is preferred for use in fishpaste products and as an ingredient in noodles. It is universally accepted that starch with longer polymer chains tends to contain higher levels of phosphate because the longer chains provide a better substrate for the phosphorylating enzyme. The phosphate content of high-amylose starches had levels up to more than five-fold compared to the wild-type starch and was generated by simultaneous inhibition of SBE A and B (Schwall et al., 2000). 
A crucial enzyme (GWD) responsible for phosphorylating starch has been identified in potatoes, and regulating the expression of this gene could change the phosphate content and viscosity of potato starch (Donath et al., 1998).

\section{d. Altered amylopectin structure}

The synthesis of amylopectin requires the concerted action of several enzymes, including starch synthases, branching enzymes and de-branching enzymes. The modification of amylopectin structure in planta by modulation of endogenous genes is complex and involves the targeting of multiple genes. Amylopectin containing more very long chains and more chains with less than 15 glucose units in length (Lloyd et al., 1999) was obtained by knocking down the activity of SSII and SSIII simultaneously. As a result of the increase in the number of short chains in amylopectin, the starch can gelatinise at a low temperature (lower than $50^{\circ} \mathrm{C}$ ) and have valuable uses in the food industry. Japonica-type rice varieties have better eating quality and their starch gelatinises at a lower temperature than indicatype rice varieties. The SSIla protein is responsible for the difference in amylopectin structure between these two varieties, which makes that the amylopectin of Japonica varieties is enriched in short chains and depleted in intermediate-sized chains (Umemoto et al., 2002).

\section{e. Granule size and number}

Starch granule size and number vary considerably across plant sources. Rice starch is relatively small (about $2 \mu \mathrm{m}$ ) while potato starches have larger granules (up to $100 \mu \mathrm{m}$ ). Starch granule size affects starch digestibility properties (Dhital et al., 2010; Noda et al., 2008; Parada and Aguilera, 2009), food quality such as noodle quality (Chen et al., 2003), and potentially crop productivity (Hofvander et al., 2004). Some studies of modified potato starches via inhibition of starch biosynthetic genes have altered granule morphology and number (Edwards et al., 1995; Jobling et al., 2002; Marshall et al., 1996). In the barley mutants (sugary 1), loss of isoamylase gene causes a profound effect on the number and timing of initiation of starch granules (Burton et al., 2002). And similar results were observed in potato tubers by down-regulation of different isoamylase isoforms (Bustos et al., 2004a). Expression of a tandem repeat of multiple starch binding domains (SBD2SBD5) from cyclodextrin glycosyltransferase of Bacillus circulans in an amylose-free potato genetic background resulted in a large number of smaller starch granules (Ji et al., 2004; Nazarian-Firouzabadi et al., 2012). 


\section{f. Other uses}

Along with the traditional bulk utilization of starch in various fields such as paper and board industry, a range of new research directions and applications for starch and its derivatives has been developed during recent years. For instance, due to the biodegradability of starch-based materials, commercially trials with starch-based packaging's for pasta (Italy) and PLA-based pots for yoghurt (Germany) have already been performed (Weber et al., 2002). Besides, potato starch granules seem, based on their ability to swell, the best source for natural microencapsulation of small molecules (Korus et al., 2003; Lii et al., 2003).

\section{Perspectives of GMO starches}

In an era towards a bio-based economy the knowledge on how to improve complex carbohydrates such as starch is essential. A deeper understanding of the starch biosynthetic pathway, how storage starch granules are formed and how the composition, size and shape can be changed and optimized for different bioproducts is of great importance for food and non-food applications.

In spite of its great importance, the development and commercialization of crops with altered starch properties using biotechnological approaches is being hampered by regulatory hurdles. The very high costs and the great deal of time needed, associated with the regulation of genetically modified crops (GMOs), are major problems. Although there is currently one GMO potato variety in the market, the Amflora, the commercialization of this variety has been challenged by farmers and environmental organizations. The development of new methods in plant breeding that would circumvent these regulatory problems would be of greatly stimulated the development of novel starches (Lusser et al., 2012). The identification of genetic marker associated with starch properties and exploitation of new mutations in tilling populations are other tools with great potential for uncovering key genes determining starch properties (Werij et al., 2012).

Another bottleneck for the production of improved starches is associated with the difficulties in predicting beforehand the effect of a (trans)gene. The understanding of mechanism by which starch granules are made in the form of dense granules would be a great step forward in the synthesis of tailored starches for different bio-based applications. 



\title{
Chapter 3
}

\section{Engineering Potato Starch with a Higher Phosphate Content}

\author{
Xuan $\mathrm{Xu}^{1,2}$, \\ Xing-Feng Huang ${ }^{1,3}$, \\ Richard G.F. Visser ${ }^{1}$, \\ Luisa M. Trindade ${ }^{1}$
}

${ }^{1}$ Wageningen UR Plant Breeding, Wageningen University and Research, P.O. Box 386. 6700AJ

Wageningen, The Netherlands.

${ }^{2}$ National Centre for Vegetable Improvement (Central China), Key Laboratory of Horticultural Plant Biology, Ministry of Education, Huazhong Agricultural University, Wuhan, 430070, China.

${ }^{3}$ Current address: Department of Chemical and Biological Engineering, Colorado State University, 1370 Campus Delivery, Fort Collins, CO 80523-1370, USA.

Submitted 


\begin{abstract}
Phosphate esters are responsible for valuable and unique functionalities of starch for industrial applications. Also in the cell phosphate esters play a role in starch metabolism, which so far has not been well characterized in the storage starch. Laforin, a human enzyme composed of a carbohydrate-binding module and a dual-specificity phosphatase domain, is involved in the dephosphorylation of glycogen. To modify phosphate content and better understand starch (de)phosphorylation in storage starch, laforin was engineered and introduced into potato (cultivar Kardal). Interestingly, expression of an (engineered) laforin in potato resulted in significantly higher phosphate content of starch, and this result was in line with the results observed in amylose-free potato genetic background (amf). Modified starches exhibited altered granule morphology and size compared to the control. About $20-30 \%$ of the transgenic lines of each series showed red-staining granules upon incubation with iodine, and contained higher phosphate content than the blue-stained starch granules. Moreover, low amylose content and altered gelatinization properties were observed in these red-stained starches. Principle component and correlation analysis disclosed a complex correlation between starch composition and starch physico-chemical properties. Ultimately, the expression level of endogenous genes involved in starch metabolism was analysed, revealing a compensatory response to the decrease of phosphate content in potato starch. This study provides a new perspective for engineering starch phosphate content in planta by making use of the compensatory mechanism in the plant itself.
\end{abstract}




\section{Introduction}

Starch is the predominant storage carbohydrate in higher plants and is a semi-crystalline composite substrate consisting of two biopolymers, amylose and amylopectin. Amylose is an amorphous, essentially linear glucan polymer with a-1,4 linked glucose residues, while amylopectin is a highly branched molecule built of $a-1,4$ linked glucose residues as backbone and 5\% a-1,6 branches (Manners, 1989). In addition to amylose and amylopectin, native starches contain small amounts of phosphate groups monoesterified to the glucose residues (Blennow et al., 2000a; Blennow et al., 2002; Kuipers et al., 1994; Tabata et al., 1975). Most of the phosphate groups are bound to the amylopectin fraction at the C-6 ( 70\%) and C-3 ( 30\%) positions of the glucose units (Bay-Smidt et al., 1994; Hizukuri and Takeda, 1970). C-6 phosphoesters are added by the glucan water dikinase (GWD1), whereas C-3 phosphoesters are catalysed by the phosphoglucan water dikinase (GWD3/PWD) (Baunsgaard et al., 2005; Kotting et al., 2005; Ritte et al., 2006; Ritte et al., 2002). Studies in Arabidopsis have indicated that phosphoglucan phosphatase starch excess 4 (SEX4) cooperates with like-SEX4 1 and 2 (LSF1 and LSF2) proteins to remove phosphate groups (Comparot-Moss et al., 2010; Kotting et al., 2009; Santelia et al., 2011). This reversible phosphorylation is essential for starch degradation in leaves. In contrast, it is not known whether reversible glucan phosphorylation occurs in storage starches, and in case it does the mechanism of phosphate removal is unknown.

Starch phosphate content differs considerably between botanical origins (Blennow et al., 2000a; Xu et al., 2014). For instance, cereal endosperm starch has less than $0.01 \%$ covalently linked phosphate, while potato tuber starch contains significantly higher phosphate content with approximately $0.5 \%$ glucose residues being phosphorylated (Blennow et al., 2000a; Tabata et al., 1975). It has been shown that phosphate content affects physico-chemical properties and the end-uses of starches, such as starch pasting properties, gel strength and clarity, stickiness and viscosity (Blennow et al., 2002). Hence, modification of starch phosphate content is a prerequisite for some industrial applications, such as internal sizing in paper making, special flocculation effects in mining, and water treatment (Ellis et al., 1998). In industry, a common method to increase starch phosphate content is chemical phosphorylation, but this process requires high amounts of energy and produces pollutant waste.

To preclude the disadvantages of post-harvest modifications of starch, many studies have focused on ways of producing starches with different phosphate content directly in planta (Xu et al., 2014; Zeeman et al., 2010). One of the alternatives is to manipulate the endogenous genes involved in starch biosynthesis through genetic engineering. Up to date, most known endogenous genes, including granule-bound starch synthase (GBSSI), glucan water dikinase (GWD1), phosphoglucan water dikinase (GWD3), starch-branching enzyme $(S B E)$, soluble starch synthases (SSS), have been overexpressed or down- 
regulated (antisense or co-suppression approaches) in plants to obtain starches with altered phosphate content (Abel et al., 1996; Frohberg et al., 2011; Lloyd et al., 1999). For instance, GWD1 has been overexpressed in rice (Frohberg, 2008), maize (LanahanMichael and Ship, 2005), wheat (Schewe et al., 2002) and barley (Carciofi et al., 2011), resulting in increased starch phosphate content. In potato, silencing of GWD1 has resulted in the reduction of both phosphate content in starch and cold-sweetening in tubers (Lorberth et al., 1998), and in wheat, the inhibition of GWD1 has led to a lower phosphate content and an increased seed yield and plant biomass (Ral et al., 2012).

Another strategy is to introduce heterologous enzymes that are able to modify starch composition and structure and in this way produce starches with novel properties ( $\mathrm{Xu}$ et al., 2014). Phosphate content can be removed by phosphatases, which are widely spread throughout different kingdoms. One of such enzymes is Laforin, a dual specificity phosphatase required for normal glycogen metabolism in vertebrates. Mutations in the laforin gene lead to Lafora disease, an autosomal recessive neurodegenerative disorder causing severe epilepsy and death (Lafora and Glueck, 1911; Minassian et al., 1999; Serratosa et al., 1999). Lafora disease is characterized by the accumulation of Lafora bodies (LBs), insoluble deposits containing high phosphate content that closely resemble amylopectin (Sakai et al., 1970). Interestingly, laforin has structural similarities to SEX4, containing a dual-specificity phosphatase (DSP) and an N-terminal carbohydrate-binding module (CBM) of the CBM20 subtype. Moreover, it is a functional equivalent of SEX4 (Gentry et al., 2007), and is unique in its ability to remove phosphate from amylopectin in vitro (Worby et al., 2006). Together, these findings suggest that laforin has a great potential to modulate phosphate content in potato starch, and could help to explore the mechanism of phosphoglucan metabolism in potato starch.

In this study, the human laforin gene, and modifications of it, were introduced into potato plants to modify phosphate content of starch. The effects of the (engineered) laforin on phosphate content, composition and properties of potato starch are presented and discussed. 


\section{Materials and methods}

\section{Constructs preparation}

Three constructs were made in this study, i) pBIN19/DSP for expression of the dualspecificity phosphatase (DSP) domain of laforin alone, ii) pBIN19/CBM20-DSP for the fulllength laforin gene expression, iii) pBIN19/SBD-DSP for the expression of the DSP domain fused to a starch-binding domain (SBD) that is derived from Bacillus circulans cyclodextrin glycosyltransferase (CGTase) (Huang et al., 2013; Huang et al., 2014; Ji et al., 2003). The potato granule bound starch synthase (GBSSI) promoter and the GBSSI transit peptide were used for tuber-specific expression and amyloplast entry of proteins, respectively. All constructs were validated by sequencing analysis.

For the assembly of the pBIN19/CBM20-DSP construct, the laforin-encoding fragment was obtained by PCR amplification with the primers 5'- TACCATGGACTACAAAGACGATGA CGATAAAACTAGTATGCGCTTCCGCTTTGGGGTG-3'(FLAG-encoding sequence underlined) and5'-GAGGATCCACGGATCTCAGTGGTGGTGGTGGTGGTGCTCGAGCAGGCTACACACA GAA-3' (HIS-encoding sequence underlined), which contained Ncol and BamHI sites at $5^{\prime}$ ends (in bold), respectively. This amplified laforin fragment, containing an N-terminal FLAG tag and a C-terminal HIS tag, was cloned into Ncol/BamHI restriction sites of pUC19/ SBD2 (S1 Fig), generating the pUC19/CBM20-DSP plasmid. After digestion of this plasmid with $\mathrm{Hpal}$ and $\mathrm{BamHI}$, the fragment was inserted into the corresponding sites of pBIN19/ SBD2 (Ji et al., 2004) to generate the pBIN19/CBM20-DSP plasmid (Fig. 1).

The linker-DSP fragment was amplified from pBIN19/CBM20-DSP by PCR, with a forward primer containing a Bg/ll site (5'- ATAGATCTTTGGTGGATGGTGTGTA $-3^{\prime}$ ) and a reverse primer containing a BamHI site (5'- GCGGATCCAGCAGCCGGATCTCAGTGGTG $-3^{\prime}$ ). The amplified fragment contained a linker, DSP sequence and HIS tag in which the linker was $117 \mathrm{bp}$ fragment of the 3 '-end of CBM20 sequence. The amplified fragment was cloned into $\mathrm{Bg} / \mathrm{II} / \mathrm{BamHI}$ restriction site of pUC19/SBD2, generating pUC19/SBD-DSP. Furthermore, the fragment was amplified from pUC19/SBD-DSP with a forward primer containing a Spel restriction site (5'-AAACTAGTATGGCCGGAGATCAGGTCAG-3') and a reverse primer containing a BamHI site ( $5^{\prime}$ - GCGGATCCAGCAGCCGGATCTCAGTGGTG -3'). After digestion of this fragment with Spel and $B a m H I$, the fragment was inserted into the corresponding sites of pBIN19/CBM20-DSP to generate the pBIN19/SBD-DSP plasmid (Fig. 1).

The pBIN19/DSP construct was made based on pBIN19/CBM20-DSP plasmid.DSP-encoding fragment was amplified by PCR with a forward primer, containing a Spel restriction site (5'-AAACTAGTCATTATTCAAGAATTCTACCAAATATCT-3') and a reverse primer, containing an Xhol site (5'- TCCTCGAGCAGGCTACACACAGAAGAA - $3^{\prime}$ ). The amplified fragment was inserted into corresponding sites (Spel and Xhol) of pBIN19/ CBM20-DSP, resulting in pBIN19/DSP plasmid (Fig. 1). 
(a) pBIN19/ㅁBM20-DSP (CD)

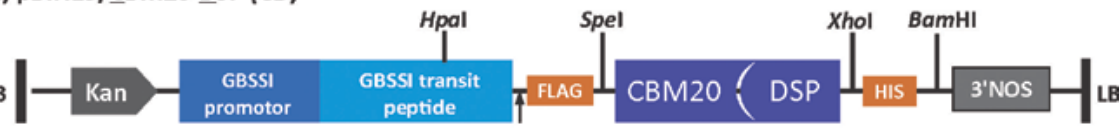

(b) pBIN19/ $\underline{B} B D-\underline{D} S P$ (SD)

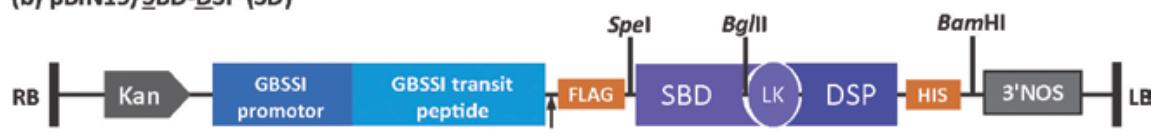

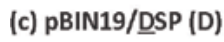

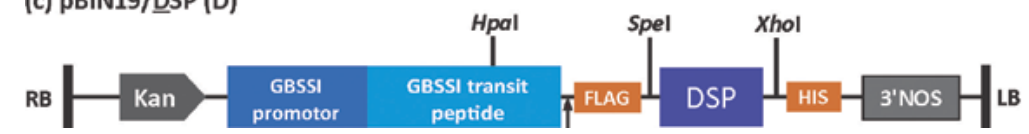

FIGURE 1. Schematic depiction of binary vector constructs used in this study. Genes were cloned in frame with GBSSI transit peptide to allow amyloplast targeting and were driven by GBSSI promoter for tuber-specific expression. CBM20 and DSP represent the carbohydrate-binding module 20 domain and a dual specificity phosphatase of laforin protein. RB and LB stand for right and left borders, respectively. SBD, LK, Kan and 3'NOS stand for starch binding domain of cyclodextrin glycosyltransferase from Bacillus circulans, linker, kanamycin resistant gene and NOS terminator, respectively. The arrow represents cleavage site of the transit peptide. FLAG and HIS are two tags for protein quantification and Hpal, Spel, Xhol, Bglll and BamHI are restriction enzymes.

\section{Transformation and regeneration}

All three binary vectors, pBIN19/CBM20-DSP, pBIN19/SBD-DSP and pBIN19/DSP (Fig. 1), were transformed into amylose-containing potato (cv. Kardal, tetraploid) and amylose free mutant (amf, diploid) according to Visser et al. (1991). About thirty independent plantlets of each construct as well as control plants (untransformed plants and transformed plants with empty vector) were multiplied to five plants by culturing nodal explants according to the procedure described in Visser et al. (1991). All plants were grown under standard greenhouse conditions $\left(16 \mathrm{~h}\right.$ light at $20^{\circ} \mathrm{C}$ and $8 \mathrm{~h}$ dark at $18^{\circ} \mathrm{C}$ ) till maturity and tubers were harvested at 18 weeks. No differences were detected between untransformed lines and transformed plantlets with empty vector, and therefore they will be further referred to as control or UT. Transformed potato plants from Kardal background were labelled CDxx, SDxx and Dxx in which CD, SD and D stand for full-length laforin protein, DSP with an SBD, and DSP alone, respectively, $x x$ represents the number of transgenic line. Transformed potato plants from amf background were labelled amfCDxx, amfSDxx and amfDxx.

\section{Starch isolation}

To minimize individual variation, tubers used for starch isolation were obtained by pooling all tubers of five plants harvested from the same transgenic clone. Starches were isolated according to the procedure described in Huang et al. (2013). 


\section{Western dot blot and protein analysis}

The amount of granule-bound fusion protein of each transformant was investigated by western dot blot analysis. About $50 \mathrm{mg}$ of starch were heated at $100^{\circ} \mathrm{C}$ for 5 min with 400 $\mu \mathrm{l}$ of a $2 \times$ SDS sample buffer containing $5 \% \beta$-mercaptoethanol (Laemmli, 1970). After cooling to room temperature, the supernatant was transferred to a 96-well format dotblot manifold (Schleicher \& Schuell, Keene, NH). The dot-blot manifold was connected to a water pump, and a vacuum was applied for 5 min until all samples were impacted on the nitrocellulose membrane (Bio-rad).

The dot blots were blocked overnight at $4^{\circ} \mathrm{C}$ in Tris-buffered saline (TBS is $20 \mathrm{mM}$ Tris, $500 \mathrm{mM} \mathrm{Nacl}$ pH 7.5) with $0.1 \%$ Tween-20 (TBST) containing $5 \%(\mathrm{w} / \mathrm{v})$ dry powdered nonfat milk. Membranes were then incubated for $2 \mathrm{~h}$ at room temperature with a 1:1000 dilution of the anti-SBD antibody (Ji et al., 2003) in 3\% non-fat milk in TBST, followed by 5 rinses in TBST. The membrane was then incubated with a 1:5000 dilution of horseradishperoxidase-conjugated anti-(rabbit lgG) (Cat \# A0545, Sigma) in TBST buffer with 3\% (w/v) of non-fat milk for $1 \mathrm{~h}$ at room temperature. After 5 times rinses in TBST, the protein was detected with a West Femto supersignal (Cat \# 34094, Thermo Scientific) (Ji et al., 2003).

To examine GBSSI abundance, proteins were stained with Coomassie brilliant blue R-250 after separation by SDS polyacrylamide gel electrophoresis (SDS-PAGE).

\section{Total phosphate content}

Total phosphate content in starch was determined essentially according to the method of Morrison (1964) with some modifications. About $20 \mathrm{mg}$ of dry starch were suspended in $250 \mu \mathrm{l}$ of $70 \%(\mathrm{w} / \mathrm{w}) \mathrm{HClO}_{4}$ and completely charred at $250^{\circ} \mathrm{C}$ for $25 \mathrm{~min}$. The solution was clarified by adding $50 \mu \mathrm{l} 30 \%(\mathrm{w} / \mathrm{v}) \mathrm{H}_{2} \mathrm{O}_{2}$ and gently boiled for $2 \mathrm{~min}$. Once the solution had cooled, water was added to a final volume of $2 \mathrm{ml}$ and $100 \mu \mathrm{l}$ of the sample was transferred into a 96-well microtiter plate, followed by adding $200 \mu$ l of colour reagent [0.75\% (w/v) $\left(\mathrm{NH}_{4}\right)_{6} \mathrm{Mo}_{7} \mathrm{O}_{24} \cdot 4 \mathrm{H}_{2} \mathrm{O}, 3 \%(\mathrm{w} / \mathrm{v}) \mathrm{FeSO}_{4} \cdot 7 \mathrm{H}_{2} \mathrm{O}$ and $0.75 \%(\mathrm{w} / \mathrm{v})$ SDS dissolved in $0.375 \mathrm{M} \mathrm{H}_{2} \mathrm{SO}_{4}$ ]. Absorbance at $750 \mathrm{~nm}$ was then measured in a Model 680 XR Microplate Reader (Bio-Rad, US), and compared to the absorption of a calibration curve to calculate the concentration in $\mathrm{nmol} \mathrm{PO}_{4} / \mathrm{mg}$ starch.

\section{Analysis of the morphology and physico-chemical properties of starch granules}

All analyses conducted in this manuscript have been performed in duplicates unless indicated otherwise.

The starch suspensions were stained with 20xdiluted Lugol's solution (1 \% I2/KI) and were then investigated with light microscopy (LM, Axiophot, Germany). Further, scanning electron microscopy (SEM) PhenomTM (FEl, The Netherlands) was used to examine the 
detailed morphology on the granule surface. About $1 \mathrm{mg}$ of starch samples were evenly distributed on a carbon tabs (Agar Scientific, UK) and mounted onto $12.70 \mathrm{~mm}$ aluminium specimen stubs (Agar Scientific, UK), followed by coating with gold using a sputter coater (EMITECH K550X; Quorum Technologies, UK). All images were digitally recorded.

Particle size distribution and gelatinization properties of starches were analysed as described in Ji et al. (2003). The onset $\left(T_{0}\right)$, peak $\left(T_{p}\right)$ and conclusion temperature $\left(T_{c}\right)$ of gelatinization and the melting enthalpy $(\Delta \mathrm{H}, \mathrm{J} / \mathrm{g})$ were determined using Differential Scanning Calorimetry (DSC).

The apparent amylose content was performed according to the procedure described in Hovenkamp-Hermelink et al. (1988).

High-performance anion-exchange chromatography (HPAEC) was used to determine amylopectin chain length distribution. Degree of polymerisation (DP) 6 - 35 was separated according to the method of Huang et al. (2013).

Starch moisture content was measured in duplicate using standard methods (AACC Approved Method 44-15.02) using oven drying at $105^{\circ} \mathrm{C}$ for 16 hours.

Starch content was determined as described by Kok-Jacon et al. (2005a).

\section{Quantitative RT-PCR}

Expression levels of (engineered) laforin from all transformants were determined in triplicate by qRT-PCR using the primers listed in S1 Table. Total RNA was extracted from potato tuber samples according to Kuipers et al. (1994) and reverse transcribed using the iScript cDNA synthesis kit from BioRad. qRT-PCR analysis was performed using CFX96 Real-Time PCR machine (BioRad). The total volume of each reaction was $10 \mu$, containing $50 \mathrm{ng}$ cDNA, $3 \mu \mathrm{M}$ of each gene-specific primer, and $5 \mu$ I SYBR Green Supermix Reagent (BioRad). All reactions were carried out using the following thermal cycling conditions: 3 min of denaturation at $95^{\circ} \mathrm{C}$, followed by 45 cycles $\left(15 \mathrm{~s}\right.$ at $95^{\circ} \mathrm{C}, 60 \mathrm{~s}$ at $60^{\circ} \mathrm{C}$ ). Using the comparative Ct method (Livak and Schmittgen, 2001), target genes were expressed relative to EF1a (Nicot et al., 2005). After normalization, data were multiplied by a factor of $10^{6}$ and then converted to log 10 . Ultimately, the resulted value $(\mathrm{v})$ was used to assign transformants to different categories: none $(N, v=0)$, low $(L, 0<v<3)$, medium $(M, 3 \leq v$ $<4)$ and high $(H, v \geq 4)$ expressers.

Five randomly-selected transgenic lines from D, CD and SD series were used for gene expression analysis of key genes involved in the starch metabolism. In total, four starchdegrading genes and seven starch-synthesizing genes were selected according to previous studies, including phosphoglucan phosphatase starch excess 4 gene SEX4 (Kotting et al., 2009), like-SEX4 gene LSF1 and LSF2 (Comparot-Moss et al., 2010; Santelia and Zeeman, 
2011), starch phosphorylase gene SP (Brisson et al., 1989), a-amylase gene AMY23 and $\beta$-amylase genes BAM1 and BAM9 (Ferreira, 2011; Zhang et al., 2013), isoamylase genes ISA1, ISA2 and ISA3 (Bustos et al., 2004b; Ferreira, 2011), starch-branching genes SBEI and SBEIl (Jobling et al., 1999; Safford et al., 1998b), soluble starch synthase genes SSSII and SSSIII (Abel et al., 1996; Edwards et al., 1995). The gene information and specific primers used in present study are listed in S1 Table. The relative expression levels were calculated using the Ef1a gene as a reference gene, employing the $2^{-\Delta \Delta C T}$ method.

\section{Statistical analysis}

Significant differences between modified starches and control samples in phosphate content and granule size were assessed by one-way analysis of variance (ANOVA). The least significant difference values were calculated at $5 \%$ probability. Statistical significances between gene expression level of transformants and that of the control were determined by using t-test. Inter-relationships between starch components and starch properties were analysed by means of Pearson correlations. All statistical analyses were performed using GenStat (16th edition). Principle component (PCA) analysis was performed using PAST software Package (Hammer et al., 2001).

\section{Results}

\section{Transgenic plants do not show visible changes in plant architecture or tuber morphology}

In total, three transgenic series containing, respectively, DSP (D), CBM20-DSP (CD) and SBDDSP (SD) were generated by introducing three constructs into potato plants. About thirty independent plants from each transgenic series and the control were multiplied and grown in the greenhouse. Plants were monitored in all stages and no significant differences were observed in plant architecture, tuber morphology nor tuber yield relative to the control plants (data not shown).

\section{(Engineered) laforin genes are expressed in transformants}

Expression of SD in transformants was examined by qRT-PCR and Western dot blot analysis. According to gene expression level, transformants were classified into four different categories: none $(\mathrm{N})$, low $(\mathrm{L})$, medium $(\mathrm{M})$ and high $(\mathrm{H})$ expressors. All SD lines showed gene expression, and $28 \%, 34 \%$ and $38 \%$ of transformants were categorized as $\mathrm{L}, \mathrm{M}$ and $\mathrm{H}$ expressors, respectively (Fig. 2a). Furthermore, the result obtained from Western dot blot using a SBD-antibody revealed that the accumulation of the protein correlated well with the 
mRNA level of the transformants (Fig. 2b). For this reason, and because no specific antibody was available for quantification of the DSP domain, the transformants from other two series, $D$ and $C D$, were only subjected to qRT-PCR analysis to determine the expression of the (engineered) laforin gene. As shown in Fig. 2a, similar to SD series, $\mathrm{M}$ and $\mathrm{H}$ expressers were predominant in these two series. For $\mathrm{D}$ series, $10 \%, 21 \%, 34 \%$ and $34 \%$ of the transformants classified as $\mathrm{N}, \mathrm{L}, \mathrm{M}$ and $\mathrm{H}$ expressors, respectively, whereas the corresponding values for the $\mathrm{CD}$ series were $7 \%, 22 \%, 44 \%$ and $26 \%$. $\mathrm{N}$-expressors were excluded from further analyses.

(a)

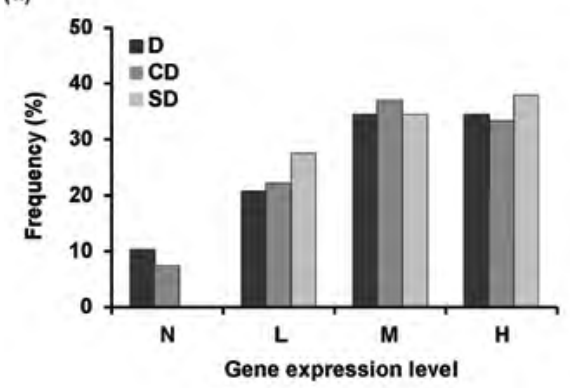

(b)

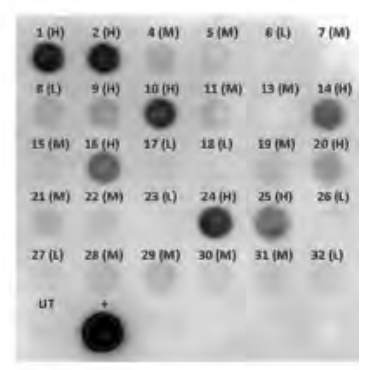

FIGURE 2. Characterization of transgenic plants. (a) Distribution of the individual transformants over the classes of the (engineered) laforin expression. The qRT-PCR analysis was performed in triplicate on all transformants, which are 27, 26 and 30 lines for D, CD and SD series, respectively. D, CD and SD represent DSP, CBM20-DSP and SBD-DSP transformants, respectively. N, L, M and H stand for none, low, medium and high expressors. (b) Accumulation level of SBD-DSP (SD) in transformants. Protein levels were determined using Western dot blot analysis with an anti-SBD antibody. The number above each dot stands for the different lines, while UT and ' + ' represent negative and positive control, respectively. The intensity of dots shows the various protein levels. The corresponding gene expression level of each line obtained from qRT-PCR is indicated between brackets. For $90 \%$ of lines a good correlation between gene expression level and protein accumulation level was found.

\section{Starch granule morphology and amylose content are altered by the introduction of an (engineered) laforin protein}

Light microscopy (LM) and scanning electron microscopy (SEM) were used to investigate how the accumulation of the (engineered) laforin protein affects the starch granule morphology. About $22 \%$ of the $\mathrm{D}$ transformants, $19 \%$ of the $\mathrm{CD}$ transformants and $27 \%$ of the SD transformants showed red granules with blue cores of varying size when stained with iodine (Fig. 3G). Further analyses indicated that both irregular bumpy granules and cracked granules coexisted in each of these modified starches (Fig. $3 \mathrm{H}$ and I), while the starches from other transgenic lines exhibited blue-stained granules with irregular bumpy surface (Fig. 3D- F) in contrast to the smooth surface observed in the control (Fig. $3 \mathrm{~A}-\mathrm{C}$ ). Based on the granule colour, transformants from each series were divided into two groups (red-stained group and blue-stained group) for further analyses. Starch with red-stained granules was occurred in transformants with different expression level of (engineered) laforin (data not shown). 
In addition, the exhibition of red-stained granules strongly suggests the reduction of amylose content in these starch granules, therefore, amylose/amylopectin ratio was determined in all modified starches and the apparent amylose content (AM\%) was calculated (Table 1). The result revealed that the red-stained starches contained much lower amylose content relative to the control, whereas all blue-stained starches did not show changes in amylose content compared to the control. The amylose level of most redstained starches was between $2 \%$ and $5 \%$, which is comparable to that of the amylosefree potato starch (Visser et al., 1997). Moreover, some lines that consisted of a mixture of red and blue-stained granules had intermediate amounts of amylose content ( 10\%), but still dramatically lower than that of the control ( 18\%).

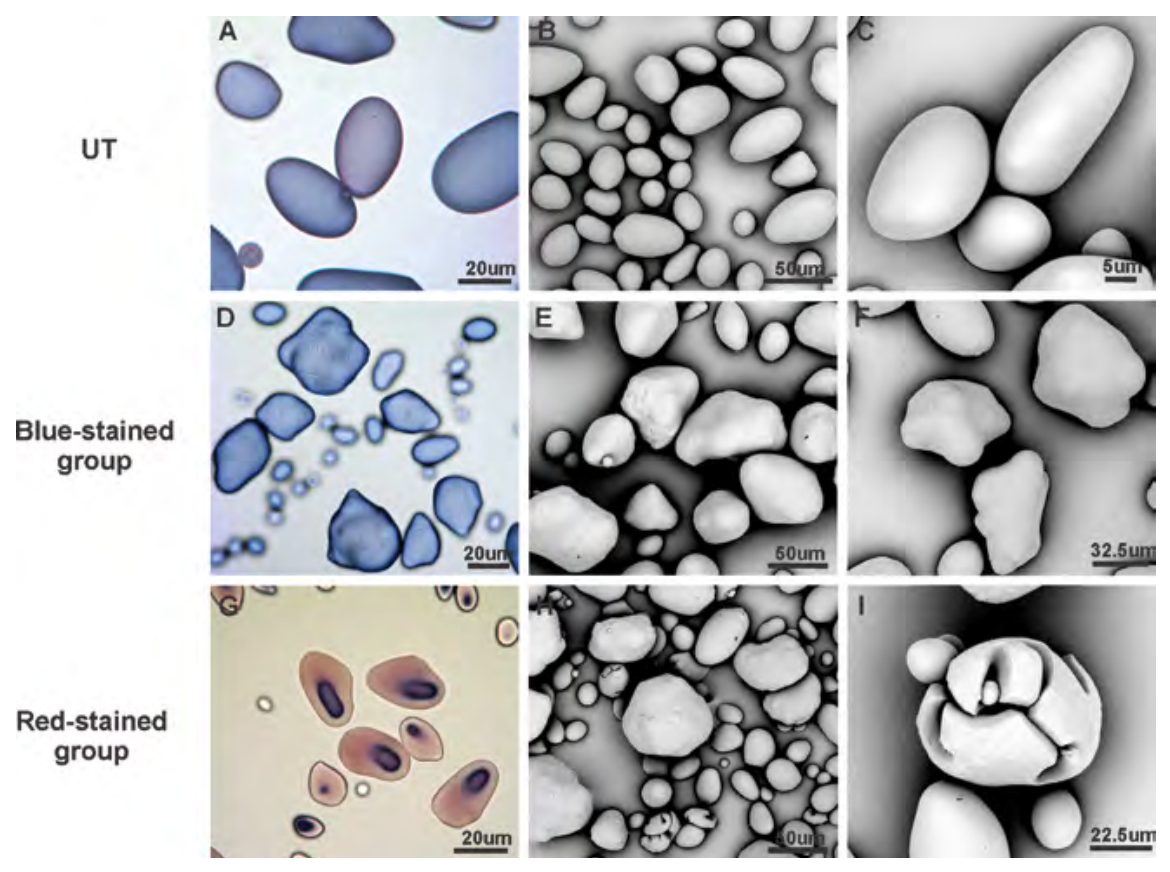

FIGURE 3. Light micrographs and SEM analyses showing starch granules morphology of control UT (A-C) and modified starches (D-I). Starch granules were stained with a $20 \times$ diluted Lugol solution for light microscopy (A, D and G). Two different morphologies were observed in the modified starches from each series. Based on the colour of stained granules, the starches were classified into two categories: blue-stained group (D-F) and redstained group (G-I).

These findings raised a question regarding the expression of GBSSI in transformants, as GBSSI is responsible for the amylose synthesis. Hence, GBSSI expression was investigated for transformants from each series, containing transformants with eight red-stained starches and seven blue-stained starches in total. The results revealed that amylose content strongly correlates with relative expression of GBSSI ( $r=0.8, p<0.001$, Fig. 4a). Furthermore, eight lines with different expression levels of GBSS/ were selected to perform 
SDS-PAGE, followed by staining with Coomassie blue. The correlation between amylose content and GBSSI abundance was observed (Fig. 4a and b). These results showed that the decrease in amylose content in red-stained starches is a consequence of GBSSI suppression in transformants.

(a)

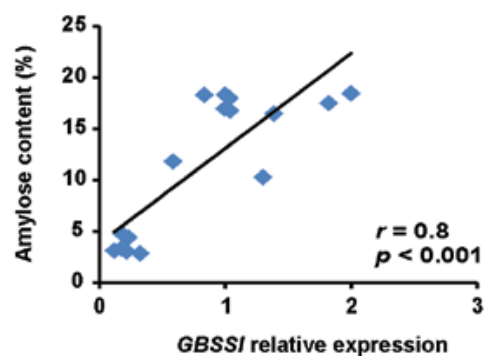

(b)

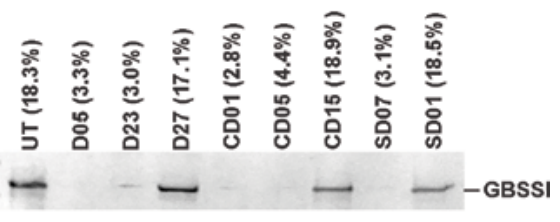

FIGURE 4. The suppression of GBSSI in transformants with red-stained granules. (a) The correlation between amylose content and the relative expression of granule-bound starch synthase I (GBSSI). The qRT-PCR analysis was performed on control UT and 5 random-selected transgenic tubers from each series, containing transformants with 8 red-stained starches and 7 blue-stained starches. (b) Western blot analysis of GBSSI abundance in starches from different transformants. The corresponding amylose content of each line is shown between brackets.

\section{Laforin expression results in higher phosphate content}

The total phosphate content in starch granules of each transgenic line was determined. Interestingly, nearly all the starches from transformants had higher phosphate content compared with that of the control, on average $19 \%$.

As shown in Fig. 5a, in all series, the phosphate content significantly increased in starches from both red-stained and blue-stained groups compared with that from control (ANOVA, $p<0.05)$. Within each series, the red-stained group had a higher phosphate content than the blue-stained group. To illustrate, the phosphate content of the red-stained group from $D$ series was $\sim 32 \%$ higher than that of the control, and $\sim 9 \%$ and $\sim 6 \%$ higher than the red-stained group from CD and SD series, respectively. In each of the three blue-stained groups, the starch phosphate content was $\sim 11 \%$ higher than that of the control, while between the three series of transformants no significant differences were observed.

In the first place, the increase in phosphate content of modified starches was linked to the increase in amylopectin content rather than to expressed laforin. However, bluestained starches containing unaltered amylose content showed a significant increase in phosphate content, which indicated that the (engineered) laforin plays a role to increase starch phosphate content. To further investigate this, the same constructs were transferred into amylose-free potato mutant (amf) and generated $\sim 30$ transformants of each transgenic series ( $a m f D, a m f C D$ and $a m f S D$ ). Starch phosphate content was measured 
for each line. Results showed that, expect for amfSD, the phosphate content in amfD and amfCD starches were $26 \%$ and $12 \%$ higher than that in control UT-amf, respectively (Fig. 5b). Overall, the increase in starch phosphate content from amf background provides confident that starch phosphate content is affected by the (engineered) laforin rather than only by GBSSI suppression.

(a)

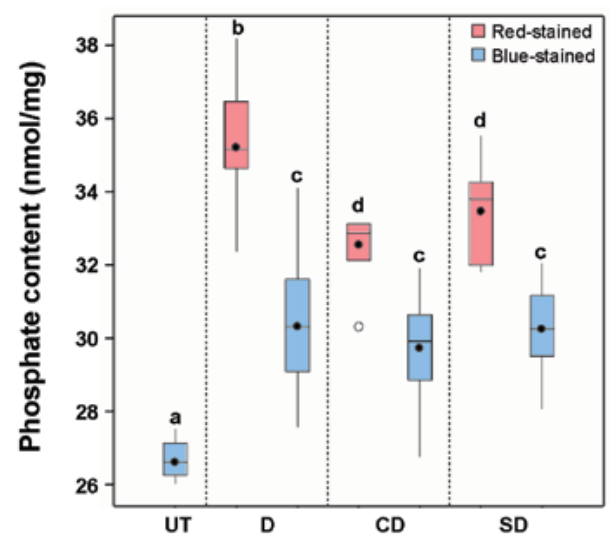

(b)

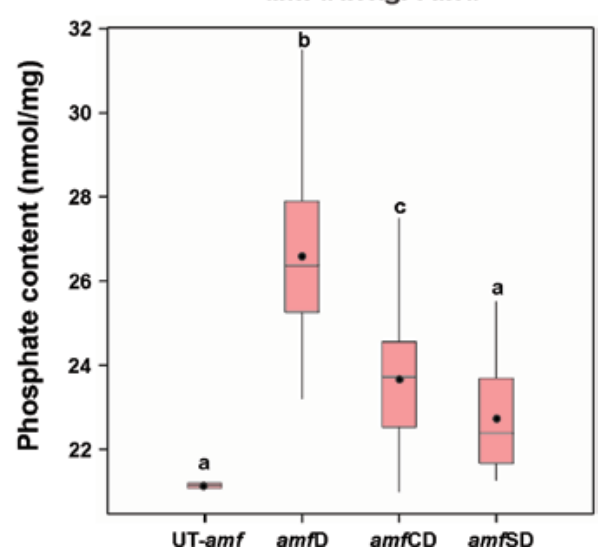

FIGURE 5. The phosphate content of starches from all transgenic series and control plants in both (a) Kardal and (b) amf backgrounds. D, CD and SD stand for transgenic series containing DSP protein, the fulllength laforin protein and SBD-DSP fusion protein, respectively. Amf represents the amylose-free potato background. All the analyses were performed in duplicate on transformants with (engineered) laforin expression, which are 24, 25, 28, 32, 25 and 29 lines for D, CD, SD, amfD, amfCD and amfSD series, respectively. Significant difference between each transgenic group and control was analysed using one-way ANOVA. Different letters (a-d) indicate statistically significant differences at $p<0.05$.

\section{Granule size and gelatinization temperature are changed in modified starches}

The median granule size ( $\mathrm{d} 50$ ) was determined for all starches by analysing granule size distribution. The transformants with CD and SD proteins had larger starch granules both in the red-stained group and blue-stained group compared to the control (Fig. 6). In particular, starch granules of red-stained starches from $C D$ series were significantly larger in size than that of blue-stained starches (ANOVA, $p<0.05$ ). By contrast, starches from the red-stained group in $D$ series did not show any significant difference in granule size. Further analyses showed that there was no significant correlation between the expression of the (engineered) laforin and granule morphology or granule size. 


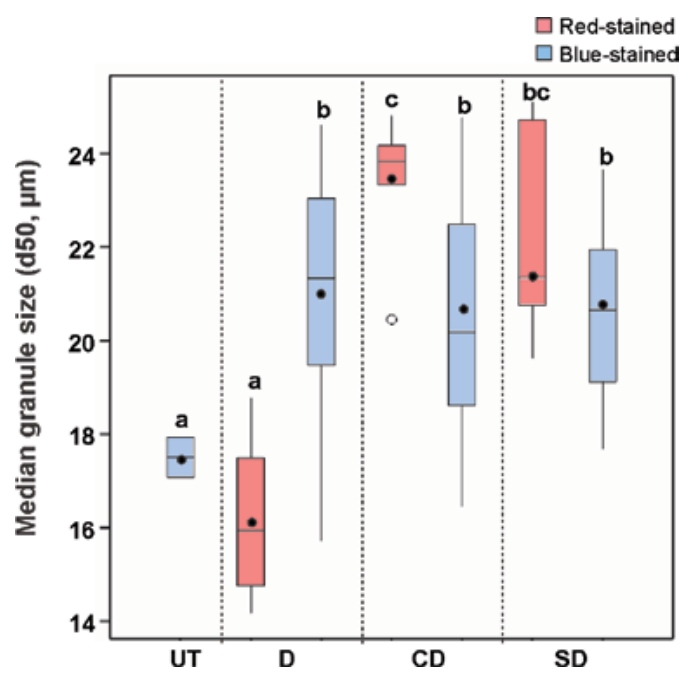

FIGURE 6. Boxplot presenting median granule size (d50) of starches from control UT and all transformants of each series. All the analyses were performed in duplicate on all transformants, which are 24,25 and 28 lines for D, CD and SD series, respectively. Significant difference between each transgenic group and control was analysed using one-way ANOVA. Different letters $(\mathrm{a}-\mathrm{c})$ indicate statistically significant differences at $p<0.05$.

DSC analysis disclosed that red-stained starches had a significantly higher $\mathrm{T}_{\mathrm{o}}$ and $\mathrm{T}_{\mathrm{p}}$ (Table 1 ), suggesting that these starches start to gelatinize and swell to a maximum level at higher temperatures compared to the control. Moreover, the gelatinization enthalpy $(\Delta H)$ of these red-stained starches was consistently lower than that of the control. Unlike the red-stained starches, the gelatinization characteristics did not differ from the control in any of the blue-stained starches.

Two representative starches of each series with blue or red-stained granules are presented. Gene-expression class (Class), starch granule colour (Colour), total phosphate content $(P)$, starch apparent amylose content $(A M)$, starch content $\left(C_{\text {starch }}\right)$, median granule size (d50), starch gelatinization temperature $\left(\mathrm{T}_{\mathrm{o}}, \mathrm{T}_{\mathrm{p}}\right.$ and $\mathrm{T}_{\mathrm{c}}$ ) and gelatinization enthalpy $(\Delta H)$ are shown. Data (mean \pm S.D.) are the average of two or three independent measurements. n.d., not detected.

Amylopectin chain length distributions of all modified starches upon debranching with isoamylase were analysed with HPAEC (chain length ranged from DP6 to DP35). No significant changes in amylopectin fine structure were observed relative to the control (data not shown). Similarly, starch content was measured for the selected starches and no consistent changes were found compared to the control (Table 1). 
TABLE 1. Summary of different starch characteristics determined for the representative modified starches and the control UT. Two representative starches of each series with blue or red-stained granules are presented. Gene-expression class (Class), starch granule colour (Colour), total phosphate content (P), starch apparent amylose content $(A M)$, starch content $\left(C_{\text {starch }}\right)$, median granule size $(d 50)$, starch gelatinization temperature $\left(\mathrm{T}_{\mathrm{o}^{\prime}} \mathrm{T}_{\mathrm{p}}\right.$ and $\mathrm{T}_{\mathrm{c}}$ ) and gelatinization enthalpy $(\Delta H)$ are shown. Data (mean \pm S.D.) are the average of two or three independent measurements.

\begin{tabular}{lcccccccccc}
\hline Line & Class Colour & $\begin{array}{c}\mathbf{P} \\
\text { (nmol/mg) }\end{array}$ & $\mathbf{A M}(\%)$ & $\begin{array}{c}\mathbf{C}_{\text {starch }} \\
(\mathbf{m g} / \mathbf{g ~ F W})\end{array}$ & $\mathbf{d 5 0}(\boldsymbol{\mu m})$ & $\mathbf{T}_{\mathbf{o}}\left({ }^{\circ} \mathbf{C}\right)$ & $\mathbf{T}_{\mathbf{p}}\left({ }^{\circ} \mathbf{C}\right)$ & $\mathbf{T}_{\mathbf{c}}\left({ }^{\circ} \mathbf{C}\right)$ & $\boldsymbol{\Delta} \mathbf{H}(\mathbf{J} / \mathbf{g})$ \\
\hline $\mathbf{U T}$ & n.d. & blue & $26.7 \pm 0.6$ & $18.3 \pm 0.2$ & $143.4 \pm 4.2$ & $17.5 \pm 0.4$ & $66.6 \pm 0.2$ & $70.6 \pm 0.2$ & $80.3 \pm 0.4$ & $20.7 \pm 0.3$ \\
D05 & $\mathrm{H}$ & red & $34.8 \pm 0.0$ & $3.3 \pm 0.1$ & $131.3 \pm 1.9$ & $14.8 \pm 1.2$ & $71.0 \pm 0.2$ & $76.0 \pm 0.1$ & $84.0 \pm 0.3$ & $17.2 \pm 0.6$ \\
D13 & $\mathrm{L}$ & blue & $31.8 \pm 0.4$ & $17.5 \pm 0.4$ & $151.5 \pm 1.2$ & $20.6 \pm 0.4$ & $68.1 \pm 0.1$ & $72.0 \pm 0.0$ & $82.2 \pm 0.6$ & $18.9 \pm 0.7$ \\
CD24 & $\mathrm{L}$ & red & $33.0 \pm 0.3$ & $4.4 \pm 0.1$ & $146.7 \pm 2.5$ & $24.2 \pm 0.2$ & $69.1 \pm 0.1$ & $73.3 \pm 0.1$ & $80.9 \pm 0.1$ & $17.4 \pm 0.2$ \\
CD28 & $\mathrm{M}$ & blue & $30.8 \pm 0.0$ & $18.0 \pm 0.5$ & $140.4 \pm 2.3$ & $22.5 \pm 0.3$ & $66.7 \pm 0.2$ & $70.7 \pm 0.2$ & $79.4 \pm 0.0$ & $18.2 \pm 0.2$ \\
SD07 & $\mathrm{M}$ & red & $35.5 \pm 0.0$ & $3.1 \pm 0.2$ & $149.5 \pm 1.0$ & $24.5 \pm 0.4$ & $69.4 \pm 0.1$ & $73.5 \pm 0.1$ & $81.2 \pm 0.2$ & $17.5 \pm 0.7$ \\
SD16 & $\mathrm{H}$ & blue & $30.1 \pm 0.1$ & $16.7 \pm 0.7$ & $140.1 \pm 0.0$ & $18.9 \pm 0.1$ & $66.1 \pm 0.3$ & $70.0 \pm 0.3$ & $79.1 \pm 0.0$ & $19.6 \pm 0.5$ \\
\hline
\end{tabular}

n.d., not detected

\section{Starches in DSP transformants are different from those in CD and SD transformants}

To disclose more information resulting from combinatorial effects of the different starch characteristics, a principal component analysis (PCA) was performed for all samples. As shown in Fig. 7, the first component (PC1) explains $~ 60.4 \%$ of the variance and separated modified starches on their phosphate content (P), amylose content (AM) and gelatinization temperatures $\left(T_{o^{\prime}} T_{p}\right.$ and $\left.T_{c}\right)$. The second component (PC2), which summarized $\sim 15.1 \%$ of observed variation, was largely influenced by the variations in the starch moisture content $(\mathrm{MC})$, gelatinization enthalpy $(\Delta H)$ and granule median size (d50). D series (red dots) was separated from CD and SD series (green and blue dots, respectively), while CD and SD modified starches did not separate from each other, suggesting that DSP alone functioned in a different way than when appended to a glucan-binding domain (CD and SD). The analysis also showed that starches from CD and SD series had larger granules and higher MC than that from D series. The modified starches with relatively higher phosphate content, circled by the dotted line, had higher gelatinization temperatures, which is consistent with the visual inspection of the data in Table 1. 


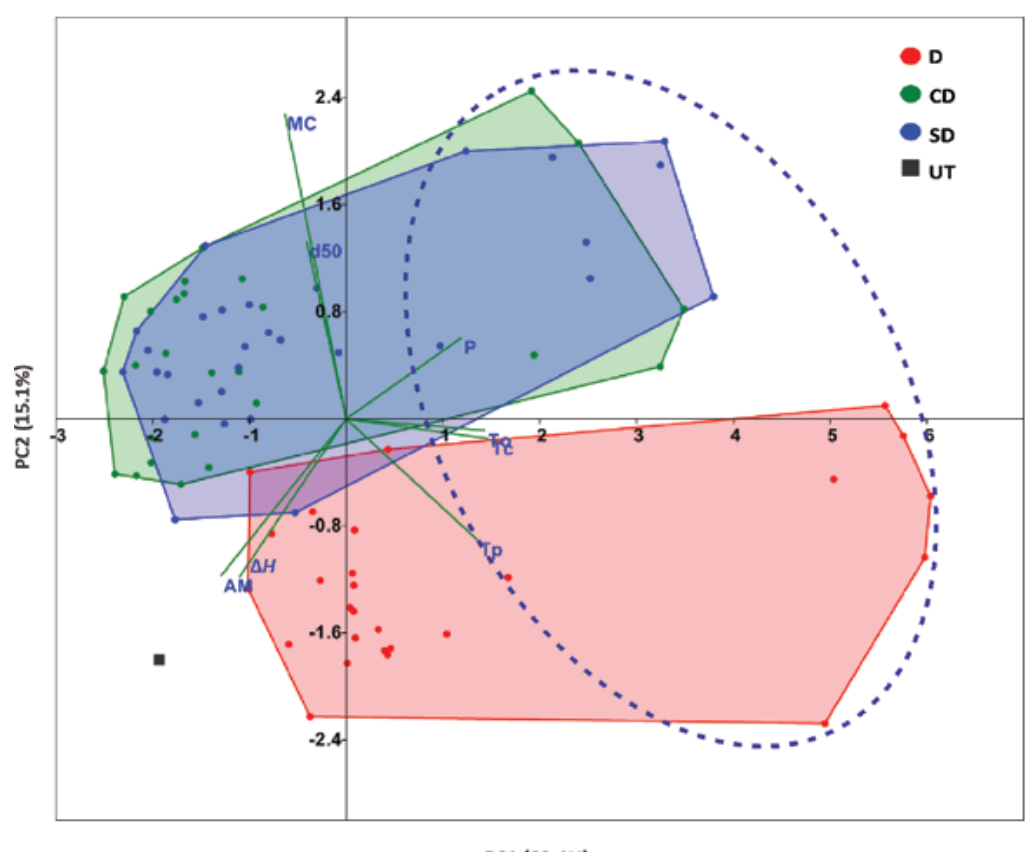

PC1 (60.4\%)

FIGURE 7. Principal components biplot displaying the classification of starches from three transgenic series based on the starch characteristics. Green vectors indicate the correlation between the different measured variables. $P$, phosphate content; $T_{o^{\prime}}$ onset temperature of gelatinization; $T_{p^{\prime}}$ peak temperature of gelatinization; $\mathrm{T}_{\mathrm{c}^{\prime}}$ conclusion temperature of gelatinization; $\mathrm{MC}$, starch moisture content; $\Delta H$, gelatinization enthalpy; AM, amylose content; $\mathrm{d} 50$, granule median size.

\section{Relationships between starch characteristics}

Correlation analyses were performed to dissect the underlying relationships between starch compositional characters and starch physico-chemical properties (Fig. 8). Strong negative correlation $(r=-0.7)$ was detected between phosphate content $(\mathrm{P})$ and amylose content (AM). Phosphate content was positively correlated $(r=0.6$ or 0.7$)$ with gelatinization temperatures $\left(T_{o^{\prime}} T_{p}\right.$ and $\left.T_{c}\right)$, and negatively correlated $(r=-0.5)$ with gelatinization enthalpy $(\Delta H)$. By contrast, AM displayed remarkably opposite correlation patterns with $\mathrm{P}$, exhibiting a negative association to $\mathrm{T}_{\mathrm{o}^{\prime}} \mathrm{T}_{\mathrm{p}}$ and $\mathrm{T}_{\mathrm{c}}(r=-0.8$ or -0.6$)$, and a positive correlation to $\Delta H(r=0.7)$. the negative correlations between starch moisture content (MC) and gelatinization temperatures $(r=-0.4$ or -0.6$)$ were observed. Furthermore, starch granule median size (d50) showed a negative correlation ( $r=-0.2$ or -0.3$)$ with $\mathrm{T}_{\mathrm{o}^{\prime}} \mathrm{T}_{\mathrm{p}}$ and $\mathrm{T}_{\mathrm{c}^{\prime}}$ but its associations with starch compositional characters ( $P, A M$ and $M C)$ were not significant. 


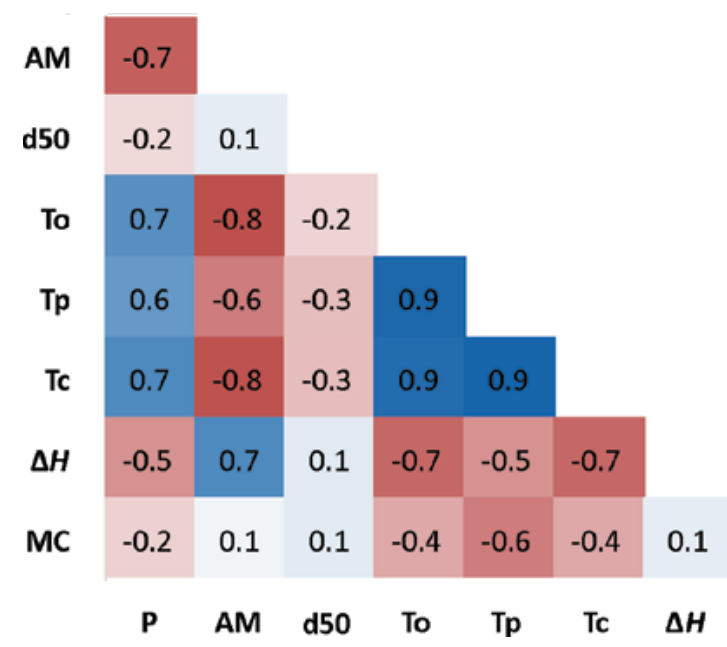

FIGURE 8. Heat-map displaying the extent and direction of correlations ( $r$ ) between starch compositional characters and starch physico-chemical properties in transgenic lines. Correlations were statistically significant at $r \geq 0.22$ and $r \leq-0.22$. Blue colours show negative correlations and red colours show positive correlations. $\mathrm{P}$, phosphate content; $\mathrm{T}_{\mathrm{o}^{\prime}}$ onset temperature of gelatinization; $\mathrm{T}_{\mathrm{p}^{\prime}}$ peak temperature of gelatinization; $\mathrm{T}_{\mathrm{c}^{\prime}}$ conclusion temperature of gelatinization; $\mathrm{MC}$, starch moisture content; $\Delta H$, gelatinization enthalpy; $\mathrm{AM}$, amylose content; $d 50$, granule median size.

\section{Laforin affects expressions of starch dikinases and hydrolases}

To understand whether these differences in starch phosphate content were caused by direct or indirect action of laforin, expression level of crucial genes responsible for starch phosphorylation was monitored by qRT-PCR on the same transformants previously selected to examine GBSSI expression. Interestingly, in general, the results displayed an increased pattern for starch dikinases, GWD1 and GWD3, in all selected transformants compared to the control (Fig. 9a). On the other hand, expression of orthologues of the Arabidopsis SEX4, LSF1 and LSF2 was not affected in transformants (S2 Fig). Further analysis revealed that the expression level of both GWD1 and GWD3 was positively correlated with starch phosphate content $(r=0.6, p<0.05$, Fig. 9b). In addition, a positive correlation ( $r=$ $0.9, p<0.001$ ) between the expression levels of both genes was found (Fig. 9c), suggesting that they are co-regulated. This result is consistent with the study of Smith et al. (2004), who found similar expression pattern for GWD1 and GWD3 genes in Arabidopsis leaves. Particularly, the expression level of these two genes was substantially higher in the $D$ transformants than that in the CD, SD and the control, which is consistent with observed higher phosphate content.

To investigate whether the expression of genes involved in starch metabolic pathways was affected in the transgenic lines, four starch-degrading and seven starch-synthesizing genes were selected and their expression was assessed by qRT-PCR analysis. Generally, 
the expression level of starch-degrading genes was altered relative to the control, but various genes showed different patterns among transformants of the three different constructs (Fig. 9b). The expression of SP in all transgenic lines was significantly higher compared to the control (t-test, $p<0.01$ or 0.001 ) regardless of the construct used. In particular, D series showed a 14-fold increase in the average expression of SP than the control, whereas a 5-fold increase was observed in CD series. Similarly, transformants from all series exhibited a significant increase in the expression of BAM9 (t-test, $p<0.001$ ). For the AMY23 expression, an increase up to 20 -fold $(p<0.05)$ was found in transfomants from $C D$ series compared with the control, but the increase in transformants from $D$ and $S D$ series was smaller. For other starch-synthesizing genes no consistent differences between transformants and the control were detected for any of the series (S2 Fig). In addition, a positive correlation was established between SP expression and GWD1 expression ( $r$ $=0.9$ ), as well as GWD3 expression ( $r=0.8$ ), indicating that these three genes are coexpressed (Fig. 9d). Taken together, introduction of the (engineered) laforin leads to the up-regulation of starch-degrading genes, but does not affect the expression of genes involved in starch synthesis. 
(a)
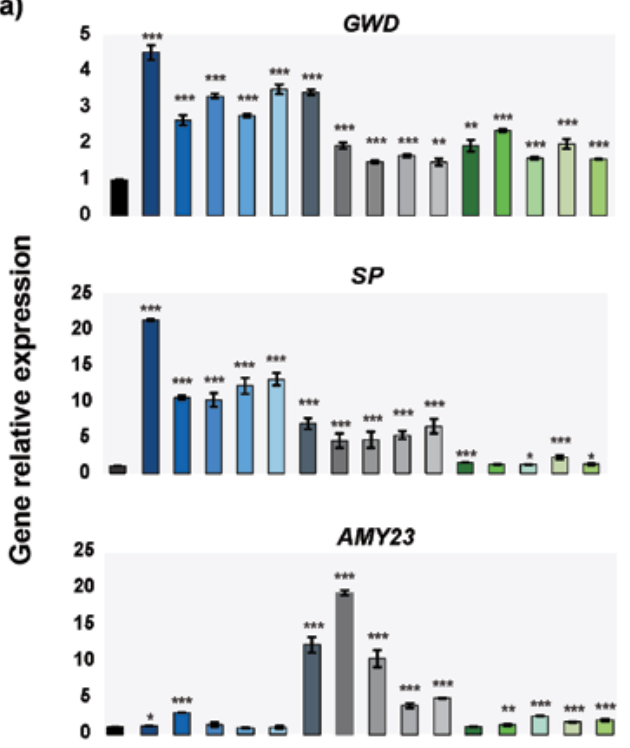

(b)

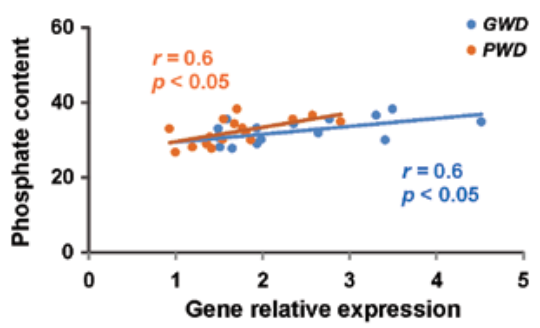

(d)

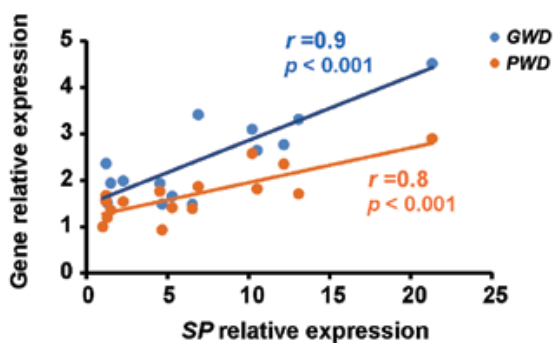

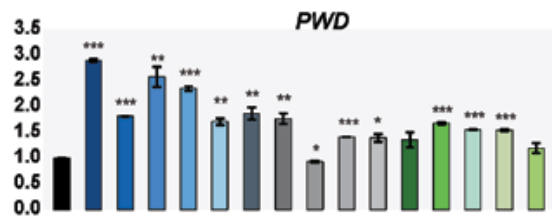

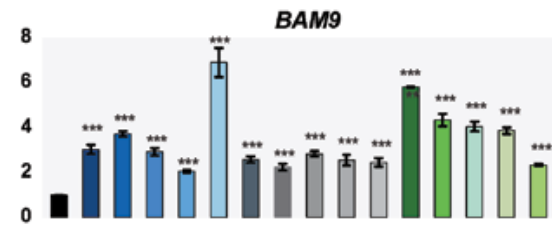

$$
\begin{aligned}
& \text { UT } \\
& \text { - D05 }=\text { CD13 } \square \text { SD04 } \\
& \text { D13 }=\mathrm{CD18} \text { 口D05 } \\
& \text { D14 } \square \mathrm{CD} 24 \text { SD07 } \\
& \text { D23 } \square \mathrm{CD25} \square \mathrm{SD16} \\
& \text { D30 שCD28 } \square \text { SD25 }
\end{aligned}
$$

(c)

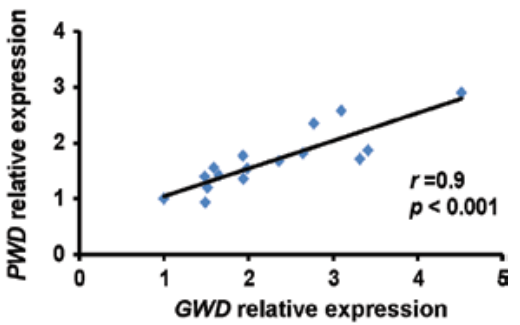

FIGURE 9. (a) The expression of the genes encoding key enzymes involved in starch metabolism and (b) correlation between starch phosphate content and gene expression and $(c, d)$ between relative expressions of different genes. qRT-PCR was performed on control UT and 5 random-selected transgenic tubers from each series, containing transformants with 8 red-stained starches and 7 blue-stained starches. The expression level of following genes were measured: glucan, water dikinase (GWD1), phosphoglucan, water dikinase (GWD3), starch phosphorylase (SP), $\beta$-amylase 9 (BAM9) and a-amylase 23 (AMY23). The values are expressed as the mean \pm S.D. from three independent measurements. Statistical significances between each starch sample and the control determined by using t-test $\left({ }^{*}, p<0.1 ;{ }^{* *}, p<0.01 ;{ }^{* * *}, p<0.001\right)$. 


\section{Discussion}

In this study, laforin, a human phosphatase gene, was engineered and introduced into potato plants to modulate phosphate content in starch granules, thus exploring the effect of phosphate content on starch properties and the mechanism of phosphoglucan metabolism in storage starch. When setting up the experiments, the idea was to generate starches with low amounts of phosphate content by expressing the (engineered) laforin gene in potato tubers. However, contrary to the expectation, the phosphate content increased substantially in starches from the transgenic lines compared with that of the control plants regardless of the construct and genetic background (Kardal and amf). This finding seems to conflict at first glance with the results of Worby et al. (2006), who found that laforin dephosphorylates potato amylopectin in vitro. Clearly, the environment in potato amyloplasts is much more intricate than that in the in vitro system, and one important difference is the presence of other endogenous genes, which may be an obstacle to detect the individual effect of laforin expression on phosphate content in potato. Therefore, we investigated whether the introduction of laforin affects the expression of other genes involved in starch phosphorylation pathway. Interestingly, the expression levels of starch phosphorylating genes, GWD1 and GWD3, were significantly increased in the different transformants. Moreover, a positive correlation was found between starch phosphate content and the transcript levels of both GWD1 and GWD3 genes (Fig. 9b). Collectively, these results demonstrate that the expression of the (engineered) laforin in potato induces starch phosphorylation via GWD1 and GWD3 and thereby leads to an increase in phosphate content in starch granules, albeit the exact mechanism by which this would work remains to be elucidated. It has been demonstrated that starch phosphate groups are essential for well-functioning starch metabolism and plant performance (reviewed in (Blennow, 2015)). It is therefore likely that a regulatory mechanism may exist to maintain certain proportional phosphate content in starch granules during starch synthesis. The phenomenon observed in this study could arise through a compensatory mechanism: where laforin removes phosphate while GWD1 and GWD3 compensate by adding extra phosphate. Hence, the phosphate content obtained in modified starch might be the result of both (engineered) laforin activity and GWD1/GWD3 activity, and each of which is difficult to quantify. A similar compensatory regulation was also proposed by Mahlow et al. (2014), who detected higher GWD3 levels in the Arabidopsis GWD1-deficiency mutants.

One other clear effect of the transformation was that about $23 \%$ of modified starches displayed red-stained color and low amylose content, and further analysis revealed that the lower content of amylose was caused by GBSSI suppression (Fig. 4). Moreover, these red-stained starches showed significantly higher phosphate content compared with that of blue-stained starches in each series (Fig. 5, Table 1). A further increase in phosphate content is likely a consequence of the decreased amylose level, based on the fact that phosphate groups are covalently bound to amylopectin but not to amylose. 
Notably, GBSSI suppression in transformants has been observed in earlier studies using similar constructs containing GBSSI promotor and transit peptide, however, this phenomenon occurred in a much lower ratio, about 3\% (Ji et al., 2003; Kortstee, 1997). Authors suggested co-suppression of GBSSI gene, and this might explain it here as well, as the promoter and transit peptide used in the constructs were derived from the GBSSI gene. However, since in this study the occurrence rate of modified starches with low amylose content is dramatically higher than the above-mentioned studies, it suggests that the upregulation of GBSS might also be a consequence of the presence of the (engineered) laforin.

Interestingly, an increase in the transcripts of starch-degrading genes in transformants was observed, but not in the starch-synthesizing genes (Fig. 9a, S2 Fig). Furthermore, a positive correlation was found between the expression of the SP and that of phosphorylating genes, GWD1 and GWD3 (Fig. 9d). These results re-enforce the view that the presence of phosphate content can stimulate starch degradation (Blennow and Engelsen, 2010; Blennow et al., 2002; Edner et al., 2007). Although one would expect to observe a decrease in starch content, we haven't been able to detect significant difference between transformants and the control.

Furthermore, CD and SD transformants did not show differences in starch characteristics, such as phosphate content, granule size, etc. These results indicate that replacing CBM20 with SBD in laforin did not affect protein behaviour in potato. Intriguingly, D transformants had a higher amount of starch phosphate content than CD and SD transformants. This might have resulted directly from the higher expression of GWD1 and GWD3 in D transformants (Fig. 9a). Another possible reason could be the different phosphatase activity when expressing $\mathrm{D}$ alone or $\mathrm{D}$ associated with a starch-binding domain. Previous studies have shown that deleting CBM20 in laforin completely abrogated the phosphatase activity of laforin (Liu et al., 2006). If this is the case, then differential turnover of starch phosphate content between D transformants and CD/SD transformants could be expected, leading ultimately to the differences in starch phosphate content.

Regardless of laforin accumulation level, the phosphate content of blue-stained starches ranged between 29 to $32 \mathrm{nmol} / \mathrm{mg}$ starch, which is similar to the highest concentration of starch phosphate found in native potato starches so far ( $35 \mathrm{nmol} / \mathrm{mg}$ starch) (Karim et al., 2007; Morrison et al., 2001; Noda et al., 2004; Suzuki et al., 1994). These data suggested that values around $30 \mathrm{nmol}$ phosphate/mg starch might be the maximal phosphate concentration in Kardal starch. To date, engineering potato starch with high phosphate content has been attained as a side effect of the alteration in amylopectin structure or amylose content, suggesting the incorporation of phosphate groups is restricted by starch structure and composition in starch granules. For instance, starch structure may have a limited number of sites where phosphorylation can take place, or it may well be that phosphorylating enzymes are not able to access or add phosphate in all possible phosphorylation sites. This is supported by the earlier studies, showing elongated and branched chains are preferably phosphorylated (Blennow et al., 2002; Schwall et al., 2000; 
Takeda and Hizukuri, 1982). A similar phenomenon has been observed in transgenic potato plants overexpressing the granule-bound starch synthase (GBSS) gene (Flipse et al., 1994; Flipse et al., 1996a). The authors found that the amylose content in transformants did not increase above the wild-type level, therefore, they suggested that there is limited space existing in starch granules to deposit amylose molecules. Collectively, it could conceivably be hypothesised that engineering potato starch for even higher phosphate starch is only possible when more phosphate groups can be deposited in the starch granules resulting from an alteration in the starch structure or starch composition.

Starch synthesis is a complex process, which is mediate by various enzymes. In this study, starch granules with altered morphology, irregular bumpy surface, and bigger size were observed in all series compared with the control. These changes are more likely to be caused by the effects of the (engineered) laforin on genes involved in starch biosynthesis/ degradation rather than due to the direct activity of the (engineered) laforin enzyme. This might be the reason why no significant correlation has been found between laforin gene expression and starch granule morphology, size and properties. The CD and SD starch granules showed a larger size than the control, which is in accord with previous observations that the introduction of starch-binding proteins in potato could alter the granule morphology and granule size (Firouzabadi et al., 2007; Huang et al., 2013; Huang et al., 2014; Ji et al., 2004).

Correlations between different starch compositional characters and starch properties have been identified. A strongly negative correlation ( $r=-0.7)$ was found between phosphate content and amylose content (in Kardal background), which is supported by previous studies (Karim et al., 2007; Kozlov et al., 2007; Noda et al., 2004; Singh et al., 2005; ViksoNielsen et al., 2001). These findings confirmed that amylose/amylopectin ratio plays a role in the regulation of starch phosphate content. The correlation between phosphate content $(P)$ and gelatinization characteristics might be an indirect effect caused by the decreased amylose content in modified starches, since we only found obvious changes on gelatinization characteristics in those starches with less amylose content (red-stained starches).

Overall, our results re-enforce the notion that alteration of starch components (amylose/ amylopectin ratio) in potato starch is an efficient way to modulate starch phosphate content in storage starch. Intriguingly, this study reveals that potato tuber starch, containing by nature already high level of starch phosphate, has obviously a strong compensatory mechanism to modulate starch phosphate content during starch biosynthesis. A better understanding of this compensatory mechanism may provide better insights on how to modify starch phosphate content in potato starch. 


\section{Supplementary data}

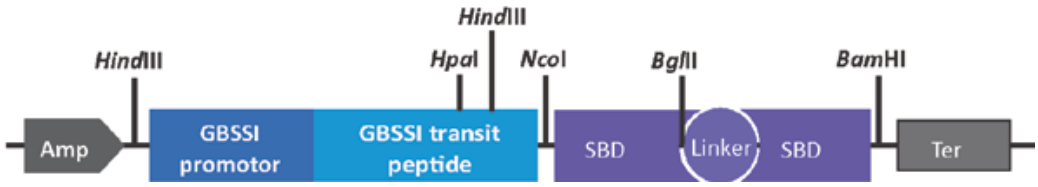

FIGURE S1. Schematic representation of pUC19/SBD2 vector. SBD, Linker, Amp and Ter stand for starch binding domain of cyclodextrin glycosyltransferase from Bacillus circulans, an artificial PT-linker, ampicillin resistant gene and terminator, respectively. To generate this construct, a sequence encoding the potato GBSSI promoter and part of GBSSI transit peptide (HindIII - HindIII) was amplified from the construct pBIN19/SBD2 (Ji et al., 2004) with primers 5'-CCAAGCTTAATACTAAAAAATGCAACAAAAT-3' and 5'-CCAAGCTTGTTAACAGCCCTTAAACCAT-3' and inserted into the corresponding sites of the pUC19 vector. The orientation of the HindIII - HindIII fragment was verified by sequencing. Subsequently, the sequence containing a SBD2 fragment and a part of GBSSI transit peptide (Hpal - BamHI) was amplified from pBIN19/SBD2 with primers 5'- CGTTAACAAGCTTGATGGGCTCCAATCAAGAACT-3' and 5'-CGGGATCCGCCAAAACAGCCAAGCTTATG-3', followed by cloning into corresponding sites of pUC19.
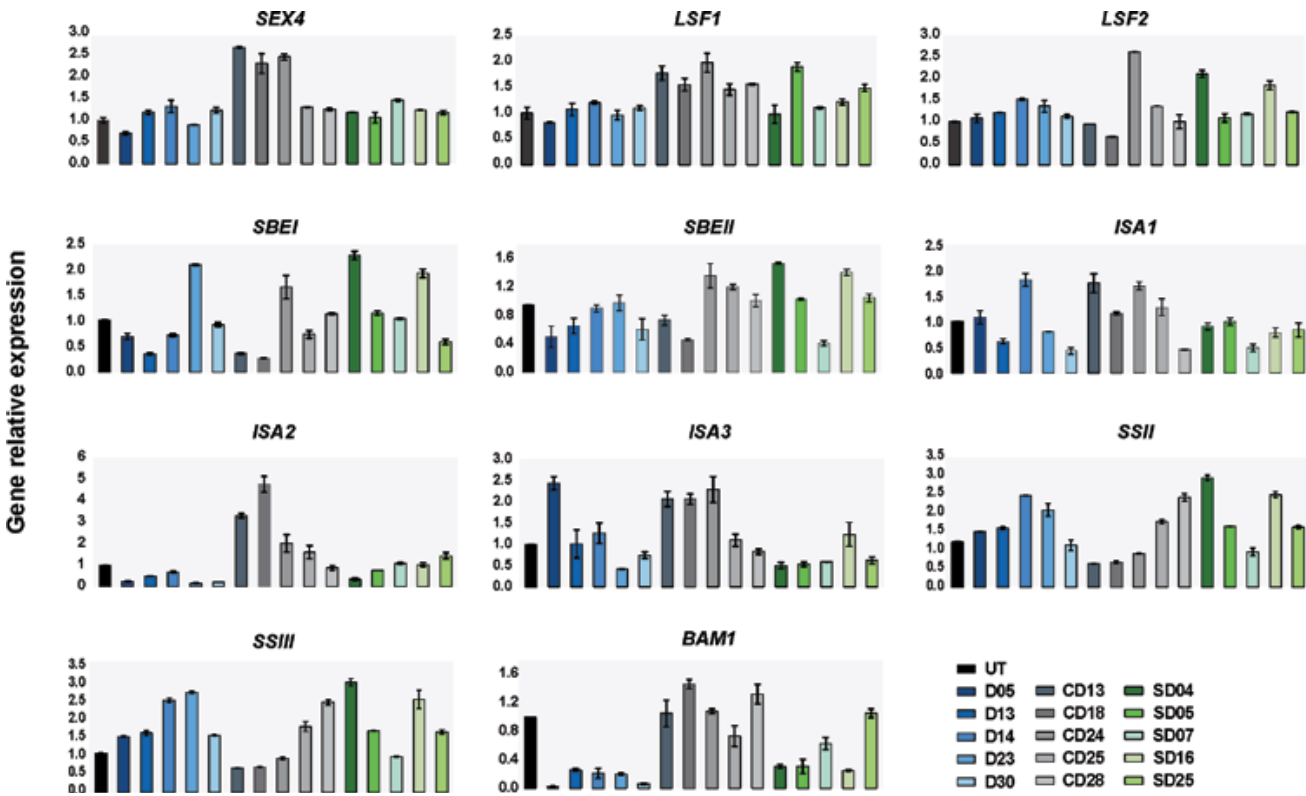

FIGURE S2. The expression level of the genes encoding key enzymes involved in starch metabolism. Include: phosphoglucan phosphatase starch excess 4 (SEX4), like-SEX4 genes (LSF1 and LSF2), starch-branching genes (SBEI and SBEII) and isoamylase genes (ISA1, ISA2 and ISA3), soluble starch synthase genes (SSSII and SSSIII) and $\beta$-amylase 1 (BAM1). The qRT-PCR was performed on control tubers (UT) and five random-selected transgenic tubers from each series, containing transformants with eight red-stained starches and seven blue-stained starches. The values are expressed as the mean \pm S.D. from three independent measurements. No consistent changes in the expression level of these genes were observed relative to the control. 


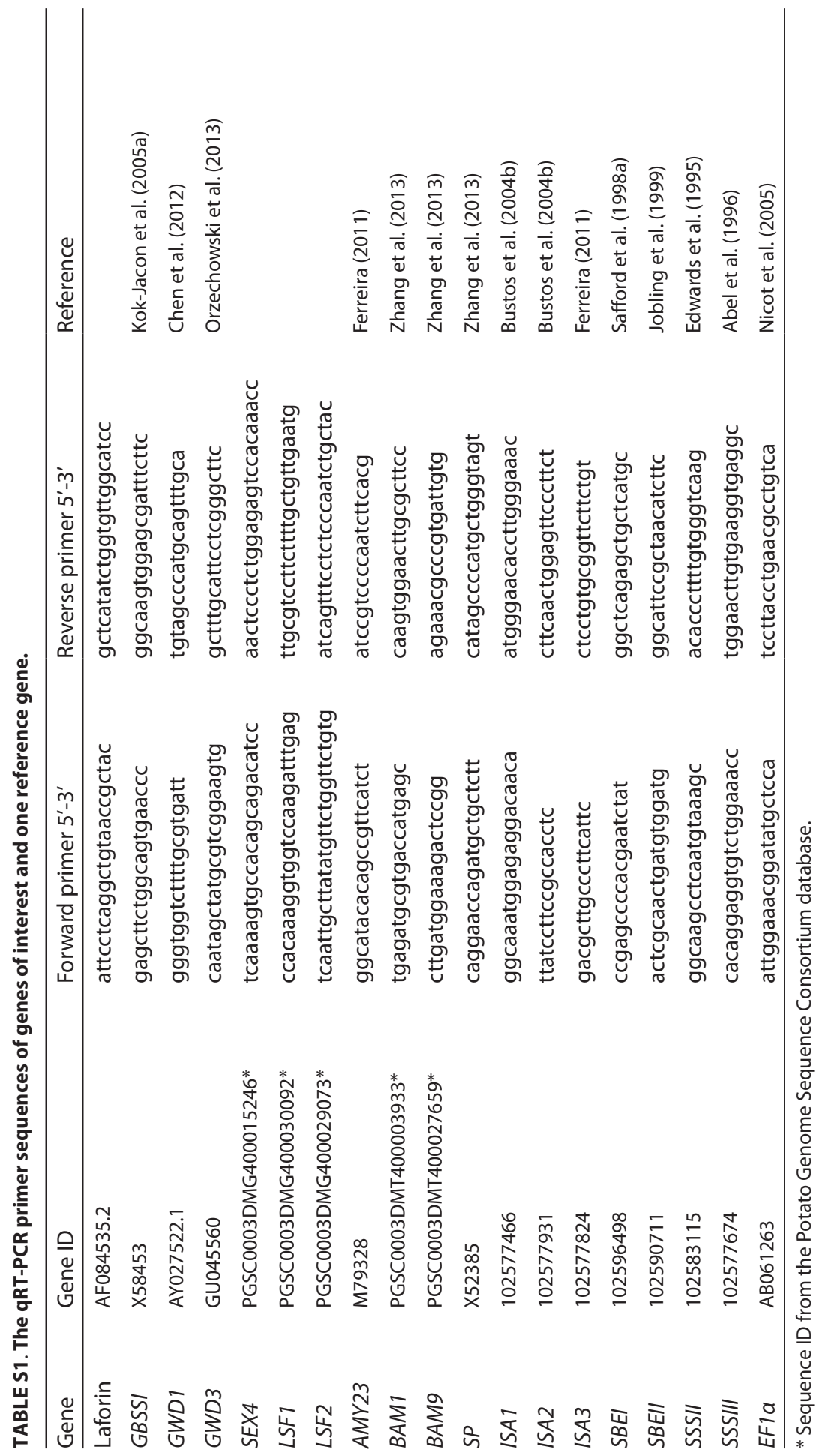




\title{
Chapter 4
}

\section{Starch phosphorylation plays an important role in starch biosynthesis}

\author{
Xuan $\mathrm{Xu}^{1,2}$, \\ Dianka Dees ${ }^{1}$, \\ Annemarie Dechesne', \\ Xing-Feng Huang ${ }^{1,3}$, \\ Richard G.F. Visser ${ }^{1}$, \\ Luisa M. Trindade ${ }^{1}$
}

${ }^{1}$ Wageningen UR Plant Breeding, Wageningen University and Research, P.O. Box 386. 6700 AJ Wageningen, The Netherlands.

${ }^{2}$ National Centre for Vegetable Improvement (Central China), Key Laboratory of Horticultural Plant Biology, Ministry of Education, Huazhong Agricultural University, Wuhan, 430070, China.

${ }^{3}$ Current address: Department of Chemical and Biological Engineering, Colorado State University, 1370 Campus Delivery, Fort Collins, CO 80523-1370, USA. 


\begin{abstract}
Starch phosphate esters are crucial in starch metabolism and render valuable functionality to starches for various industrial applications. A glucan, water dikinase (GWD1) was overexpressed in tubers of two different potato genetic backgrounds: amylosecontaining line Kardal and amylose-free mutant amf. In both backgrounds, this resulted in two effects, a number of plants showed effects of overexpression of GWD1, while others lines exhibited co-suppression of GWD1, thereby generating two series of starches with broad-scale variation in phosphate content (2-33 and 0-28 nmol/mg starch in Kardal and amf background, respectively). A comprehensive and systematic analysis was conducted on these two series of starches, with the aim to investigate in detail the effects of starch phosphate content on starch characteristics. The results revealed that starch phosphate content strongly influenced starch morphology, amylose content, starch fine structure, gelatinization characteristics and freeze-thaw stability of starch gels, but no changes in starch content and starch digestibility were found between starches from transformants and control lines. The possible role of starch phosphate groups in starch granule formation and effects of these on starch properties are discussed. Further analyses on the expression level of genes involved in starch metabolism and sugar-starch conversion suggested that starch phosphorylation regulated starch synthesis by controlling the carbon flux into starch while simultaneously modulating starch-synthesizing genes.
\end{abstract}




\section{Introduction}

Starch is a major component contributing to yield in a variety of crop plants and also a sustainable feedstock for many industrial applications. It is principally composed of two glucose polymers, amylose and amylopectin. Amylose is mainly a linear molecule with a-1,4 linked glucose residues, while amylopectin is a highly branched molecule with a $-1,6$ linkages. Besides these polymers, phosphate esters are covalently bound to starch granules, providing specific functionalities to starches and thus making these more suitable for different industrial applications. These phosphate esters are mainly present in the amylopectin chains and monoesterified at both the C-6 ( 70\%) and C-3 ( 30\%) positions of glucose units (Blennow et al., 2000a; Hizukuri et al., 1970). The level of starch phosphorylation depends on plant organ and species. In potato tubers, storage starches contain up to $33 \mathrm{nmol}$ phosphate $/ \mathrm{mg}$ starch, whereas the phosphate content in transitory starch does not exceed $0.1 \%$ (Blennow et al., 2000a). The presence of high phosphate content in potato starch is in part responsible for the high swelling power and stablepaste properties, thereby broadening the end-use of potato starches to a wide variety of applications in food and non-food industries (Jobling, 2004).

Two enzymes have been identified as being responsible for starch phosphorylation: glucan, water dikinase (GWD1) (Ritte et al., 2002) and phosphoglucan, water dikinase (GWD3/PWD) (Baunsgaard et al., 2005; Kotting et al., 2005). GWD1 and GWD3 selectively catalyse the formation of starch phosphate monoesters at the C-6 and C-3 positions of glucose units, respectively (Ritte et al., 2006), with GWD3 activity dependent on that of GWD1 (Baunsgaard et al., 2005). It has been shown that GWDs are widely distributed in the plant kingdom including Arabidopsis leaves, leaves and tubers of potato, roots of sweet potato and yam, cereal endosperm of barley and maize, and banana fruit, suggesting their omnipresent role in plant metabolism (Blennow, 2015; Lorberth et al., 1998; Ritte et al., 2000).

To date, the essential role of starch phosphorylation in starch degradation has been demonstrated in both leaves and tubers. The direct evidences were obtained in transgenic plants deficient in GWD1, showing starch excess phenotypes in the leaves of Arabidopsis and potato and the repression of cold-sweetening in potato tubers (Lorberth et al., 1998; Yu et al., 2001a). In transitory starch, phosphate groups can bring disturbance into the crystalline building blocks of starch granules and by that facilitate the access of degrading enzymes at the granule surface, thereby stimulating starch degradation (Blennow and Engelsen, 2010; Ritte et al., 2007). On the other hand, less recognition has been given to the effects of starch phosphorylation on starch synthesis. A recent study in Arabidopsis thaliana has shown that the suppression of GWD1 inhibited starch synthesis in the light phase, indicating that starch phosphorylation can stimulate starch synthesis (Skeffington et al., 2014). However, much remains to be learned with respect to the exact metabolic role of starch phosphorylation especially in storage starch. 
Previous studies have reported the effect of the down-regulation of GWD1 on starch composition and properties (Kozlov et al., 2007; Vikso-Nielsen et al., 2001); however, these studies were conducted on a small sample size and thus strongly limiting conclusions. Therefore, further study in detail is required. In particular, such information could facilitate the understanding of the role of starch phosphorylation in the regulation of starch granule assembly and starch metabolism in storage starch, and generating tools for tailoring starch in planta.

The present study extends previous studies by exhaustively characterizing potato transformants with a large range of starch phosphate content from two genetic backgrounds. We present a comprehensive and systematic analysis to investigate the impact of starch phosphorylation on starch characteristics. The roles of starch phosphorylation in starch granule formation, biosynthetic pathway and on starch properties are discussed.

\section{Materials and methods}

\section{Plasmid construction}

For the assembly of the pBIN19/GWD1 construct, the GWD1 encoding fragment was obtained by PCR amplification with the primers 5'- TACCATGGACTACAAAGACGATGAC GATAAAACTAGTATGAGTAATTCCTTAG-3'(FLAG-encoding sequence underlined) and5'GAGGATCCACGGATCTCAGTGGTGGTGGTGGTGGTGCTCGAGCATCTGTGGTCTTGTC-3' (HIS-encoding sequence underlined), which contained $\mathrm{Ncol}$ and $\mathrm{BamHI}$ sites at $5^{\prime}$ ends (in bold), respectively. This amplified GWD1 fragment, containing an N-terminal FLAG tag and a C-terminal HIS tag, was cloned into Ncol/BamHI restriction sites of pUC19/SBD2 (Supplementary Fig. S1), generating the pUC19/GWD1 plasmid. After digestion of this plasmid with $\mathrm{Hpal}$ and $\mathrm{BamHI}$, the fragment was inserted into the corresponding sites of pBIN19/SBD2 (Ji et al., 2004) to generate the pBIN19/GWD1 plasmid (Fig. 1).

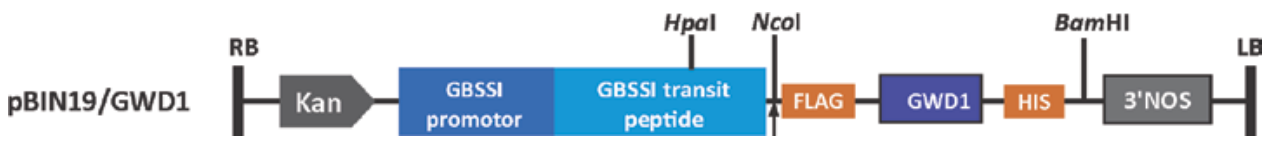

FIGURE 1. Schematic depiction of the construct PBIN19/GWD1. GWD1 was cloned in frame with GBSSI transit peptide to allow amyloplast targeting and was driven by GBSSI promoter for tuber expression. RB and LB stand for right and left borders, respectively. Kan and 3'NOS stand for kanamycin resistant gene and NOS terminator, respectively. The arrow represents the cleavage site of the transit peptide. FLAG and HIS are two tags for protein quantification and $\mathrm{Hpal}, \mathrm{NcOl}$ and $\mathrm{BamHI}$ are restriction enzymes. 


\section{Transformation and regeneration}

pBIN19/GWD1 was introduced into both amylose-containing (Kardal) and amylose-free (amf) potato genetic backgrounds via Agrobacterium tumefaciens transformation, yielding the series KDG and amfG in which KD and G represent Kardal background and GWD1, respectively. After kanamycin selection, about thirty independent transgenic plants of each series as well as control plants (untransformed plants and transformed plants with empty vector) were multiplied to five plants and grown under standard greenhouse conditions $\left(16 \mathrm{~h} \mathrm{light}\right.$ at $20^{\circ} \mathrm{C}$ and $8 \mathrm{~h}$ dark at $18^{\circ} \mathrm{C}$ ). Tubers were harvested the end of the life-cycle. No differences were detected between untransformed lines and transformed plantlets with empty vector, therefore they were further referred to as control or UT.

\section{Starch isolation}

To minimize individual variation, tubers from five plants from the same clone were pooled together, and starch isolation was performed according to the procedure described in Huang et al. (2013).

\section{Analysis of starch characteristics}

All analysis conducted in this manuscript have been performed in duplicate unless indicated otherwise.

Starch suspensions were stained with 20xdiluted Lugol's solution ( $\left.1 \% \mathrm{I}_{2} / \mathrm{KI}\right)$ and then investigated with light microscopy (LM, Axiophot, Germany). Further, scanning electron microscopy (SEM) Phenom ${ }^{\mathrm{TM}}$ (FEl, The Netherlands) was used to examine the detailed morphology of starch granule surfaces. About $1 \mathrm{mg}$ of starch samples were evenly distributed on carbon tabs (Agar Scientific, UK) and mounted onto $12.70 \mathrm{~mm}$ aluminium specimen stubs (Agar Scientific, UK), followed by coating with gold using a sputter coater (EMITECH K550X; Quorum Technologies, UK). All images were digitally recorded.

Starch samples were embedded in rapid setting Araldite and $1.5 \mu \mathrm{m}$ thick sections were cut using an Ultramicrotome (Ultracut E, Reichert-Jung) according to the procedure of (Ridout et al., 2002). The sections were stained with $20 \times$ diluted Lugol's solution $\left(1 \% \mathrm{I}_{2} / \mathrm{KI}\right)$ and then investigated with light microscopy (LM, Axiophot, Germany).

Particle size distribution was measured with a Coulter Multisizer II (Beckman-Coulter, UK) equipped with a $200-\mu \mathrm{m}$ aperture tube. Approximately $10 \mathrm{mg}$ of starch samples were suspended in a particle-free electrolyte (ISOTON ${ }^{\circledR}$ II, Beckman-Coulter, UK), and then analysed by counting 50,000 particles with coincidence factor set at $10 \%$.

Gelatinization properties of both modified starches and control starches were analysed by Differential Scanning Calorimetry (DSC), with the Perkin-Elmer Pyris 6 (Perkin-Elmer, The 
Netherlands). The starch samples were adjusted to $80 \%$ moisture content in a stainlesssteel pan by adding an appropriate amount of water according to the dry matter content of the starch samples. The pan was hermetically sealed followed by equilibration of the starch samples and the water at room temperature overnight. The samples were scanned in the temperature range from $40^{\circ} \mathrm{C}$ to $100^{\circ} \mathrm{C}$ at a rate of $10^{\circ} \mathrm{C} / \mathrm{min}$, using an empty pan as the reference. The onset $\left(T_{o}\right)$, peak $\left(T_{p}\right)$ and conclusion temperature $\left(T_{c}\right)$ of gelatinization and the melting enthalpy $(\Delta H, \mathrm{~J} / \mathrm{g})$ were determined.

The apparent amylose content was measurement according to the procedure described in Hovenkamp-Hermelink et al. (1988).

High-performance anion-exchange chromatography with pulsed amperometric detection (HPAEC-PAC) was used to determine amylopectin chain length distribution (CLD) on granule surfaces and whole granules. CLD of the whole granule was analysed according to the method of Huang et al. (2013). CLD of the granule surface was determined with the same protocol, but without the solubilisation of starches in the first step.

Starch content was determined as described by Kok-Jacon et al. (2005a).

Total phosphate content in the starch was determined according to the method of Morrison (1964) with some modifications. Twenty mg of dry starch were suspended in $250 \mu \mathrm{l}$ of $70 \%(\mathrm{w} / \mathrm{w}) \mathrm{HClO}_{4}$ and completely charred at $250{ }^{\circ} \mathrm{C}$ for $25 \mathrm{~min}$. The solution was clarified by adding $50 \mu \mathrm{l} 30 \%(\mathrm{w} / \mathrm{v}) \mathrm{H}_{2} \mathrm{O}_{2}$ and gently boiled for $2 \mathrm{~min}$. After cooling to room temperature, water was added to a final volume of $2 \mathrm{ml}$ and $100 \mu \mathrm{l}$ of the sample were transferred into a 96-well microtiter plate, followed by adding $200 \mu$ of colour reagent $\left[0.75 \%(\mathrm{w} / \mathrm{v})\left(\mathrm{NH}_{4}\right)_{6} \mathrm{Mo}_{7} \mathrm{O}_{24} \cdot 4 \mathrm{H}_{2} \mathrm{O}, 3 \%(\mathrm{w} / \mathrm{v}) \mathrm{FeSO}_{4} \cdot 7 \mathrm{H}_{2} \mathrm{O}\right.$ and $0.75 \%(\mathrm{w} / \mathrm{v}) \mathrm{SDS}$ dissolved in $0.375 \mathrm{M} \mathrm{H}_{2} \mathrm{SO}_{4}$ ]. Absorbance at $750 \mathrm{~nm}$ was then measured in a Model 680 XR Microplate Reader (Bio-Rad, US), and compared to the absorption of a calibration curve to calculate the concentration in $\mathrm{nmol} \mathrm{PO}_{4} / \mathrm{mg}$ starch.

Starch digestibility was determined according to the method of Warren et al. (2015) with some modifications. Fifty milligrams of starch were suspended in $4.95 \mathrm{~mL}$ of acetate buffer $\left(0.2 \mathrm{M}\right.$, pH 6, containing $200 \mathrm{mM} \mathrm{CaCl}_{2}$ and $\left.0.5 \mathrm{mM} \mathrm{MgCl}_{2}\right)$, followed by incubation with $50 \mu \mathrm{l}$ enzyme solution containing $10 \mathrm{U}$ a-amylase (porcine pancreas, Sigma) and $5.6 \mathrm{U}$ amyloglucosidase (Aspergillus niger, Megazyme) for $2 \mathrm{~h}$ at $37^{\circ} \mathrm{C}$. Aliquots $(200 \mu \mathrm{l})$ were centrifuged and subjected to HPAEC. The amount of glucose was calculated and presented in $\mu \mathrm{g} / \mathrm{mg}$ dry starch.

Starch freeze-thaw stability of starch gels was measured in triplicates according to the method described in Jobling et al. (2002). 


\section{RNA-sequencing (RNA-seq) and quantitative RT-PCR (qRT-PCR) analysis}

Mature tubers were sampled from five plants from the same clone. Samples were frozen in liquid $\mathrm{N} 2$ and stored in $-80^{\circ} \mathrm{C}$ freezer until RNA isolation was conducted. Total RNA was extracted from potato tuber samples according to Kuipers et al. (1994). cDNA was synthesized from $1 \mu \mathrm{g}$ of total RNA, previously treated with RNase-free DNase I (Invitrogen), by using the iScript ${ }^{\mathrm{TM}}$ CDNA Synthesis Kit (Bio-Rad). Quantitative real time PCR (qRT-PCR) was performed on all transformants and control plants to determine the expression level of GWD1. The total volume of each reaction was $10 \mu \mathrm{l}$, containing $50 \mathrm{ng}$ CDNA, $3 \mu \mathrm{M}$ of each gene-specific primer, and $5 \mu \mathrm{l}$ SYBR Green Supermix Reagent (BioRad). All reactions were carried out in triplicate using CFX96 Real-Time PCR machine (BioRad) with the following thermal cycling conditions: $3 \mathrm{~min}$ of denaturation at $95^{\circ} \mathrm{C}$, followed by 45 cycles $\left(15 \mathrm{~s}\right.$ at $95^{\circ} \mathrm{C}, 60 \mathrm{~s}$ at $60^{\circ} \mathrm{C}$ ). The elongation factor 1 -a gene (EF1a) was used as the reference to normalize gene expression across the samples because it was reported to be relatively stable in expression (Nicot et al., 2005). Target genes were expressed relative to EF1a using the comparative Ct method (Livak and Schmittgen, 2001). Gene-specific primers were designed with Primer-3-Plus software (Untergasser et al., 2007a) and are listed in Supplementary Table S1.

Tubers of two transformants from each background containing high- and low-phosphate starches and control lines were subjected to the RNA-seq. RNA quality and quantity was measured using the NanoDrop 2000 (Thermo Scientific, Waltham, MA, USA) and Agilent 2100 Bioanalyzer (Agilent, Santa Clara, CA, USA). mRNA was enriched with the oligo(dT) magnetic beads and fragmented into $\sim 200$ bp fragments. Then first strand of cDNA was synthesized using random hexamer-primer. The second strand was subsequently synthesized by adding buffer, dNTPs, RNase $\mathrm{H}$ and DNA polymerase I into the reaction. The double strand cDNA was then purified with magnetic beads, followed by end reparation and 3 '-end single nucleotide A (adenine) addition. Finally, sequencing adaptors were ligated to the fragments and the ligation products were enriched by PCR amplification. The quality and quantity of the library were verified using Agilent 2100 BioAnalyzer system and ABI StepOnePlus Real-Time PCR System. The products of RNA-seq libraries were sequenced with Illumina HiSeqTM 2000 (SE50). Construction of RNA sequencing library, sequencing and bioinformatics analyses were performed by BGI Tech (BGl, Hongkong).

Based on the RNA-seq data, starch metabolic genes with differential expression were chosen to further investigate on six transformants with low-phosphate starches and six transformants with high-phosphate starches from both backgrounds. These selected genes were starch phosphorylase (SP), starch synthases (SSII and SSIII), starch branching gene (SBEII), sucrose synthase 4 (SUSY4), ADP-glucose pyrophosphorylase (AGPase), Glc6P/phosphate translocators (GPT1 and GPT2) and invertase (INV), granule-bound starch synthase I (GBSSI), fructokinase (FK), a-amylase (AMY1 and $A M Y 2)$ and $\beta$-amylase 
(BAM). All target genes were expressed relative to EF1a (Nicot et al., 2005) and determined in triplicate by using the comparative Ct method (Livak and Schmittgen, 2001). The gene information and specific primers used in this study are listed in Supplementary Table S1.

\section{Statistical analysis}

Significant differences in phosphate content, GWD1 expression level, amylose content, starch thermal properties and the expression of starch metabolic genes were assessed using t-test. Inter-relationships between starch components and starch properties were analysed by means of Pearson correlations. All statistical analyses were performed using GenStat (16th edition).

\section{Results}

\section{Modified starches show a wide variation in phosphate content}

About thirty independent plants from each genetic background fror each of the constructs were grown in the greenhouse and mature tubers were harvested at the end of the lifecycle. No significant differences in plant architecture, tuber morphology or tuber yield were detected in transgenic plants relative to control plants.

Starch phosphate content was measured for starches from all transformants and control plants. Modified starches exhibited broad-scale variation in phosphate content in both $\mathrm{KD}$ and amf backgrounds, 2-33 and 0-28 nmol/mg starch, respectively (Fig. 2). Based on the phosphate content, modified starches were further divided into two groups (GI and GII) for each background. Gl group contained starches with higher phosphate content relative to the control, whereas GIl group contained starches with lower phosphate content compared to the control. In the KD background, KDGI group consisted of half (14 out of 29) of the transformants, and the other half of the transformants comprised the KDGII group. Four out of thirty transformants from amf background were classified into amfGI group, while the others were classified into amfGll group.

As shown in Fig. 2, regardless of background, starches from both Gl and Gll groups showed a significant change in phosphate content compared to their respective control (t-test, $p$ $<0.001$ ). Particularly, the average phosphate content of starches from KDGl group was $\sim 11 \mathrm{nmol} / \mathrm{mg}$ starch, which corresponded to $41 \%$ of the starch phosphate content in UT-KD lines ( $27 \mathrm{nmol} / \mathrm{mg}$ ). The average phosphate content of starches from amfGl group was $\sim 7 \mathrm{nmol} / \mathrm{mg}$ starch, corresponding to $\sim 43 \%$ of the starch phosphate content in the UT-amf lines ( $17 \mathrm{nmol} / \mathrm{mg})$. In KDGll group, the average phosphate content of starches was increased to $31 \mathrm{nmol} / \mathrm{mg}$ starch, while that of starches from amfGll groups was 
increased to $20 \mathrm{nmol} / \mathrm{mg}$ starch. Taken together, these series of starches with broad-scale variation in phosphate content open up the possibility of investigating the effect of starch phosphate content on starch composition and properties with a good resolution.

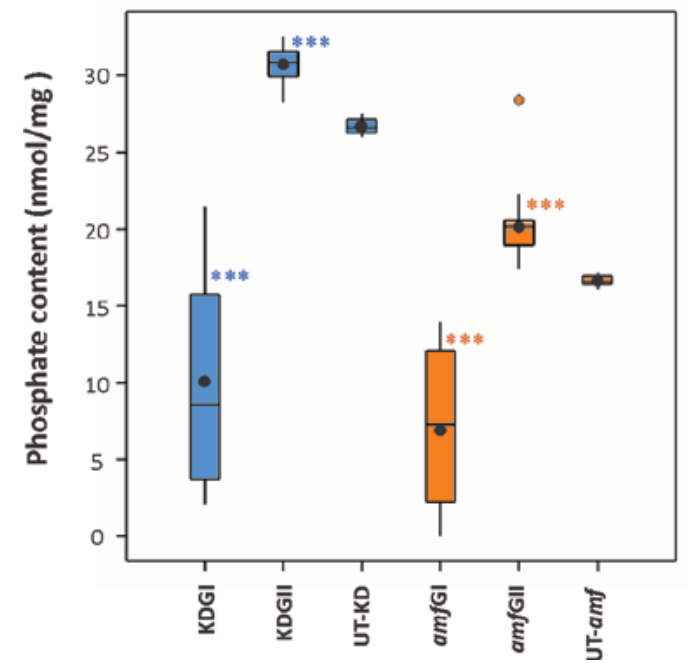

FIGURE 2. Box-plot representing the phosphate content of starches from all transformants in both genetic backgrounds and respective untransformed controls (UT-KD and UT-amf). KDGI and amfGI stand for the groups that contain starches with lower phosphate content than the corresponding controls, while amfGI and amfGll are the groups that contain starches with lower phosphate content than the controls. Boxes in the plot include values in the $25 \%-75 \%$ interval, internal lines represent the median, colour-filled circles indicate the mean, black circles indicate outliers and bars represent extremes. All the measurements were performed in duplicates. Significant differences determined by using t-test $(* * *, p<0.001)$.

\section{Alterations in starch phosphate content are caused by modulation of GWD1 expression}

To examine whether the alteration of starch phosphate content was a consequence of changes in GWD1 expression, expression of the GWD1 gene was verified in all transformants using qRT-PCR. As shown in Fig. 3a, GWD1 expression levels of KDGI transformants was on average more than 2-fold lower than in the control, while some KDGIl transformants showed about 20\% increase in GWD1 expression compared to the control. Similarly, this significant decrease was found in the GWD1 expression of amfGI transformants relative to the control (t-test, $p<0.001)$. Furthermore, a positive correlation between starch phosphate content and GWD1 expression level was observed (Fig. 3b, $r$ $=0.7$ and 0.8 in the KD and amf background, respectively). This result indicates that the differences in starch phosphate content are likely to be directly a consequence of changes in GWD1 expression. 
(a)

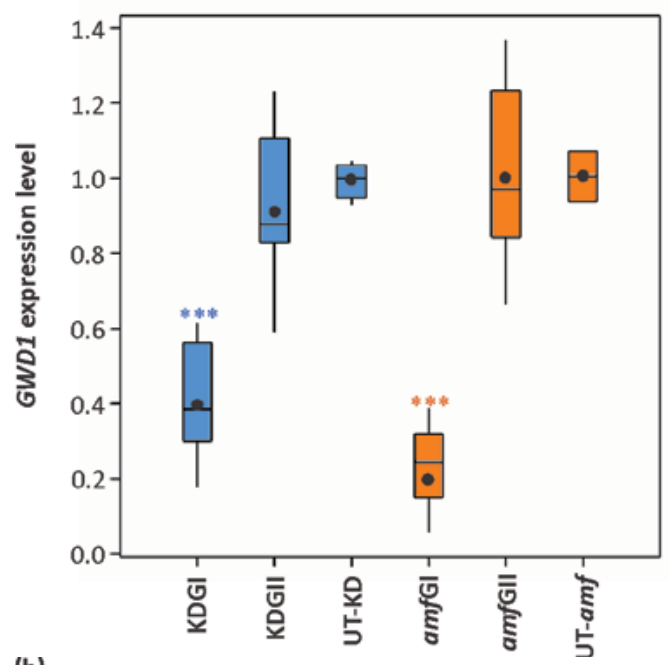

(b)

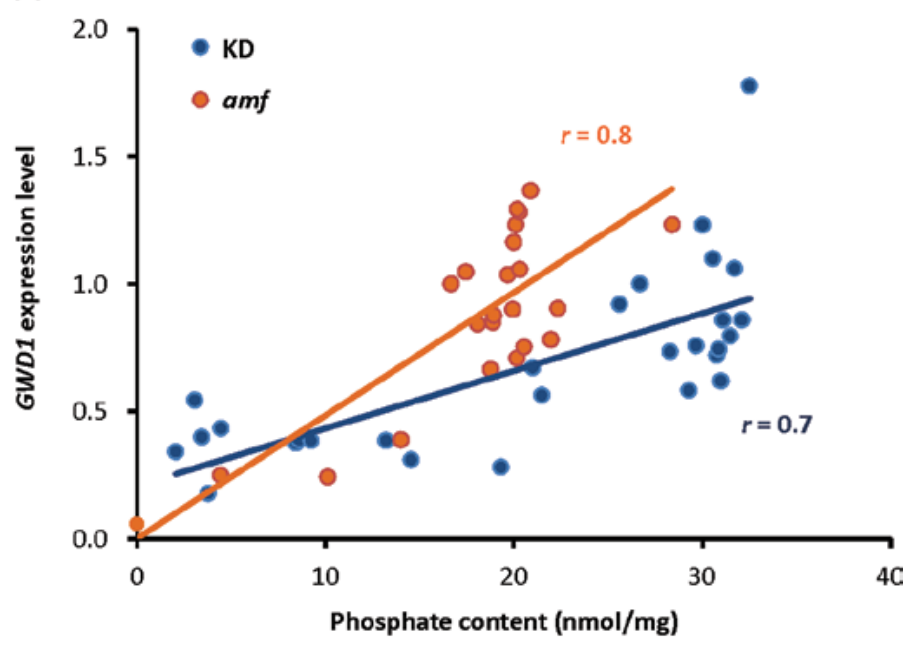

FIGURE 3. GWD1 expression level of all transformants and control plants. (a) Box-plot representing the GWD1 expression levels of transformants from KDGl, KDGll, amfGl, amfGll and control plants (UT-KD and UT-amf). Boxes in the plot include values in the $25 \%-75 \%$ interval, internal lines represent the median, black circles indicate the mean and bars represent extremes. All the measurements were performed in duplicates. Significant differences determined by using t-test (***, $p<0.001)$. (b) The correlation between GWD1 expression and phosphate content in both backgrounds. 


\section{Modified starches show changes in granule morphology}

To investigate starch granule morphology, light microscopy (LM) and scanning electron microscopy (SEM) analyses were performed on both modified and control starches. KDGI starch granules with low phosphate content (less than $10 \mathrm{nmol} / \mathrm{mg}$ starch) showed a uniform rounded shape with fissures (Fig. 4B and H), while UT-KD granules exhibited an oval shape (Fig. 4A and E). The clustered "bubbles" and grooves protruded at the surface of KDGl starch granules (Fig. 4K) contrasted with the smooth surface observed in UT-KD granules (Fig. 4J). Interestingly, the extent of morphological changes correlates with starch phosphate content, as fissures diminish in granules with higher phosphate content and ultimately disappear when phosphate content was more than $15 \mathrm{nmol} / \mathrm{mg}$ starch. Starch granules from KDGIl group showed irregular bumpy surfaces compared to the control starch (Fig. 4C, I and L). The internal structure of the starch granules was examined by sectioning granules and visualizing under LM. Severe internal cracks were present in the KDGI starch granules and less distinct growth rings were observed compared with the ones of control and KDGIl starch granules (Fig. 4D, E and F). Moreover, the protrusions of granules from both KDGI and KDGIl were only placed on the granule surface.

In the amf background, the shape of amfGl starch granules was more spherical compared with that of control and amfGll starches (Fig. 4P, Q and R). The distribution of growth rings in control and amfGll granules were apparently identical (Fig. 4M and O). The width of the rings was decreased from the hilum to the periphery and they were much greater at the distal end than those at the proximal end. By contrast, growth rings in amfGl granules were displayed in the pattern of concentric circles and ring width was similar at the distal and proximal ends, showing that the hilum was more central than those in the control and amfGll granules (Fig. 4N). 

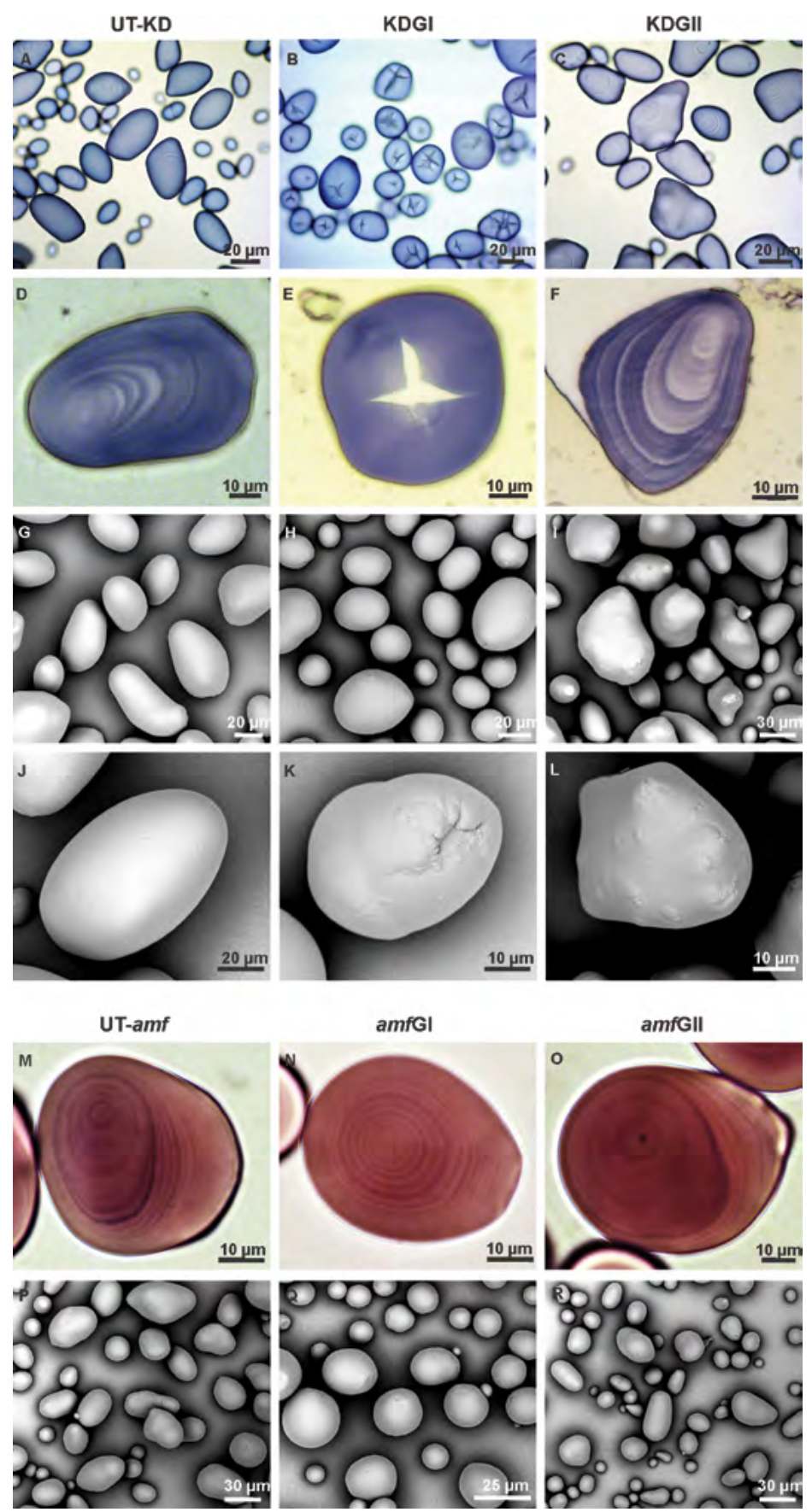

FIGURE 4. Light micrographs and SEM analyses showing the starch granules morphology of UT-KD (A, $D$, G and J), KDGI (B, E, H and K), KDGII (C, F, I and L), UT-amf (M and P), amfGI ( $N$ and Q) and amfGII (O and R). Section images showing the granule internal structure of UT-KD (D), KDGI (E) and KDGII (F). Starch granules were stained with a 20x diluted Lugol solution for light microscopy. 


\section{Starch fine structure is altered in modified starches}

We investigated the chain length distribution of all starches using native and solubilized starches. After debranching with isoamylase, the soluble glucans released from starches were analysed using HPAEC-PAC. Regardless of the background, glucan chains obtained from granule surface did not show differences between transformants and control (data not shown).

By contrast, significant differences in chain length distribution of solubilized starches were observed between GI starches and GIl starches. The distribution profile of KDGI starches exhibited a significant decrease in DP6 (t-test, $p<0.001$ ) and increase in DP 8-12 and DP 16-35 compared to the KDGII starches (Fig. 5a). Similarly, amfGl starches showed a significant lower proportion of DP6 (t-test, $p<0.001$ ) and a higher proportion of DP 8-29 compared to amfGll starches (Fig. 5b).

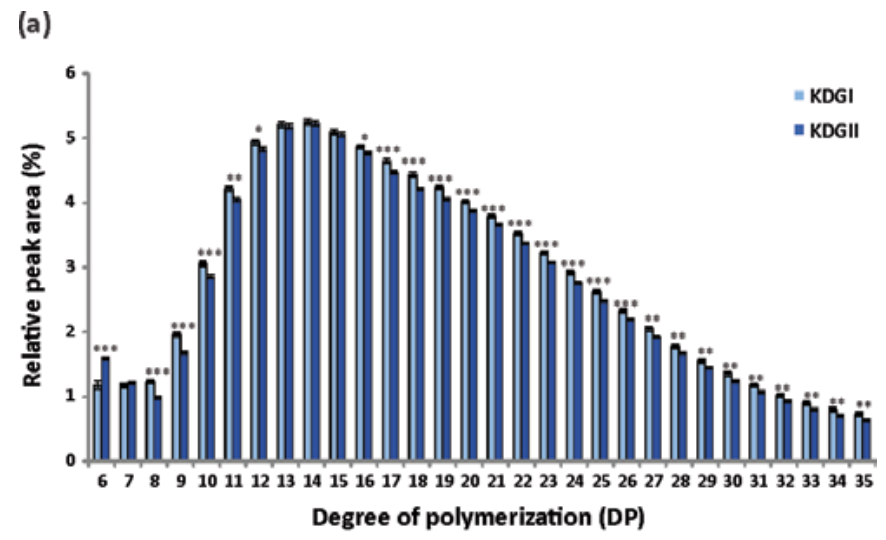

(b)

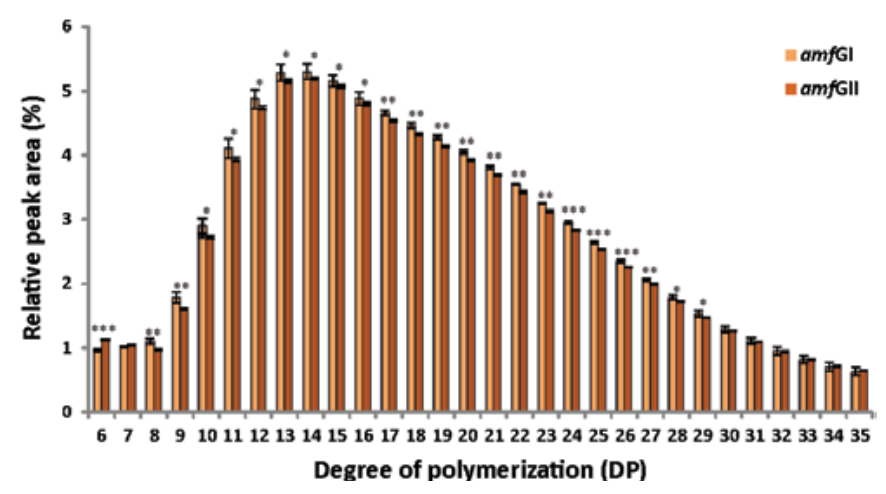

FIGURE 5. Characteristics of starch fine structure. Chain length distribution profile with DP 6-35 of debranched starches from (a) KD background and (b) amf background. Values are expressed as the mean \pm S.E. In each background, significant differences between the group with low phosphate content (KDGl and amfGI) and the group with high phosphate content (KDGII and amfGII) determined by using t-test $\left(^{*}, p<0.05, * *, p<0.01\right.$ and $* * *, p<0.001)$. 


\section{Modified starches exhibit alterations in starch composition and properties}

Amylose content (AM \%) was determined for all starches. The results showed that the amylose content varies slightly among all KD starches, ranging from 18 to $23 \%$ (Table 1, Supplementary Fig. S2). A strong negative correlation ( $r=-0.9)$ was found between amylose content and phosphate content in KD starches (Fig. 6, Supplementary Fig. S2), indicating that starch phosphate content affects the ratio of amylose/amylopectin content in starch. Starch content was not consistently different between transgenic and control tubers in both backgrounds (Table 1). The average starch content of UT-KD and UT-amf were $19 \%$ and $14 \%$, respectively, which was comparable to that of their respective transgenic plants.

The thermal properties of starch granules were studied with DSC. Gelatinization temperatures ( $T_{o}, T_{p}$ and $\left.T_{c}\right)$ significantly increased in starches with low phosphate content in both backgrounds (Table 1). The gelatinization enthalpies $(\Delta H)$ were lower in KDGI starches compared to KDGII and control starches, while it did not significantly differ in starches from amf background. Moreover, correlation analyses revealed that $\mathrm{T}_{\mathrm{o}^{\prime}} \mathrm{T}_{\mathrm{p}}$ and $\mathrm{T}_{\mathrm{c}}$ were negatively correlated $(-0.7 \leq r \leq-0.5)$ with starch phosphate content regardless of the background, while they were positively correlated $(0.4 \leq r \leq 0.7)$ with amylose content in the KD background.

TABLE 1. Summary of different starch characteristics determined for the representative modified starches in both backgrounds. Six representative starches of each background with low, medium and high level of phosphate content are presented. Total phosphate content (P), amylose content (AM), starch content (SC), median granule size (d50), starch gelatinization temperature $\left(\mathrm{T}_{\mathrm{o}^{\prime}}, \mathrm{T}_{\mathrm{p}}\right.$ and $\left.\mathrm{T}_{\mathrm{c}}\right)$ and gelatinization enthalpy $(\Delta H)$ are shown. Data (mean \pm S.D.) are the average of two or three independent measurements. Significant differences between modified starch and control determined by using t-test $\left({ }^{*}, p<0.05 ;{ }^{* *}, p<0.01\right.$ and $\left.{ }^{* * *}, p<0.001\right)$.

\begin{tabular}{|c|c|c|c|c|c|c|c|c|}
\hline Lines & $\begin{array}{l}\text { P } \\
(\mathrm{nmol} / \mathrm{mg})\end{array}$ & AM (\%) & SC (\%) & $\mathrm{d} 50(\mu \mathrm{m})$ & $\mathrm{T}_{0}\left({ }^{\circ} \mathrm{C}\right)$ & $\mathrm{T}_{\mathrm{p}}\left({ }^{\circ} \mathrm{C}\right)$ & $\mathrm{T}_{\mathrm{c}}\left({ }^{\circ} \mathrm{C}\right)$ & $\Delta H(J / g)$ \\
\hline UT-KD & $26.7 \pm 0.6$ & $18.8 \pm 0.8$ & $18.8 \pm 0.3$ & 17.5 & $66.6 \pm 0.3$ & $70.5 \pm 0.3$ & $80.1 \pm 0.6$ & $19.9 \pm 1.3$ \\
\hline KDGI-14 & $2.1 \pm 0.0^{* * *}$ & $23.1 \pm 0.0^{* * *}$ & ${ }^{*} 16.6 \pm 0.2^{*}$ & 19.8 & $68.6 \pm 0.0^{* * *}$ & $71.1 \pm 0.0^{*}$ & $81.6 \pm 0.0^{* *}$ & $16.9 \pm 0.4^{*}$ \\
\hline KDGI-5 & $3.1 \pm 0.0^{* * *}$ & $22.8 \pm 0.8^{* * *}$ & ${ }^{*} 15.4 \pm 0.1^{* *}$ & 22.8 & $68.5 \pm 0.1^{* * *}$ & $71.3 \pm 0.4^{* *}$ & $81.8 \pm 0.1^{* *}$ & $14.3 \pm 0.1^{* * *}$ \\
\hline KDGI-1 & $13.2 \pm 0.0^{* * *}$ & $21.1 \pm 0.3^{* *}$ & $17.5 \pm 0.2^{*}$ & 21.5 & $67.5 \pm 0.3^{* * *}$ & $71.7 \pm 0.4^{* * *}$ & $80.7 \pm 0.7$ & $17.0 \pm 0.2^{* *}$ \\
\hline KDGI-25 & $14.6 \pm 0.1^{* * *}$ & $21.1 \pm 0.3^{* *}$ & $19.6 \pm 0.3$ & 19.5 & $67.0 \pm 0.1$ & $71.0 \pm 0.2$ & $80.1 \pm 0.2$ & $19.8 \pm 0.1$ \\
\hline KDGII-6 & $32.1 \pm 1.0^{*}$ & $18.7 \pm 0.6$ & $10.9 \pm 0.3^{* *}$ & 25.7 & $65.3 \pm 0.1^{* * *}$ & $69.4 \pm 0.1^{* * *}$ & $77.9 \pm 0.1^{* * *}$ & $18.3 \pm 0.1$ \\
\hline KDGII-17 & $32.5 \pm 1.2^{*}$ & $18.7 \pm 0.3$ & $17.1 \pm 0.4$ & 24.6 & $66.5 \pm 0.3$ & $70.4 \pm 0.1$ & $79.7 \pm 0.8$ & $19.2 \pm 1.1$ \\
\hline UT-amf & $16.7 \pm 0.3$ & $3.6 \pm 0.2$ & $14.3 \pm 0.9$ & 12.1 & $72.3 \pm 0.3$ & $76.6 \pm 0.3$ & $84.4 \pm 0.4$ & $17.2 \pm 0.6$ \\
\hline amfGl-12 & $0^{* * *}$ & $3.9 \pm 0.1$ & $11.2 \pm 0.1^{* *}$ & 13.1 & $76.3 \pm 0.2^{* * *}$ & $80.2 \pm 0.1^{* * *}$ & $88.8 \pm 0.6^{* * *}$ & $17.0 \pm 1.5$ \\
\hline amfGl-9 & $4.4 \pm 0.5^{* * *}$ & $4.6 \pm 0.6^{*}$ & $9.6 \pm 0.7^{* * *}$ & 13.5 & $75.2 \pm 0.1^{* * *}$ & $78.8 \pm 0.0 * * *$ & $87.1 \pm 0.4^{* * *}$ & $16.7 \pm 0.1$ \\
\hline amfGl-28 & $14.0 \pm 0.2^{* *}$ & $* 3.8 \pm 0.0$ & $11.2 \pm 0.5^{*}$ & 13.6 & $73.6 \pm 0.0^{* * *}$ & $77.8 \pm 0.2^{* *}$ & $86.6 \pm 0.1^{* * *}$ & $16.8 \pm 0.7$ \\
\hline amfGll-16 & $17.4 \pm 0.2^{* *}$ & $4.2 \pm 0.6$ & $9.2 \pm 1.1^{* * *}$ & 11.4 & $73.9 \pm 0.0^{* * *}$ & $78.1 \pm 0.0^{* * *}$ & $86.5 \pm 0.1^{* * *}$ & $16.9 \pm 0.4$ \\
\hline$a m f G \| l-3$ & $21.5 \pm 0.5^{*}$ & $* 3.8 \pm 0.0$ & $10.1 \pm 0.4^{* *}$ & 15.3 & $73.7 \pm 0.2^{* * *}$ & $77.9 \pm 0.3^{* *}$ & $86.1 \pm 0.5^{* *}$ & $17.3 \pm 0.4$ \\
\hline amfGIl-24 & $28.4 \pm 0.2^{* * *}$ & $4.1 \pm 0.1 *$ & $13.1 \pm 0.3$ & 10.5 & $72.6 \pm 0.0$ & $78.6 \pm 0.0^{* * *}$ & $87.0 \pm 0.1^{* * *}$ & $16.7 \pm 0.3$ \\
\hline
\end{tabular}




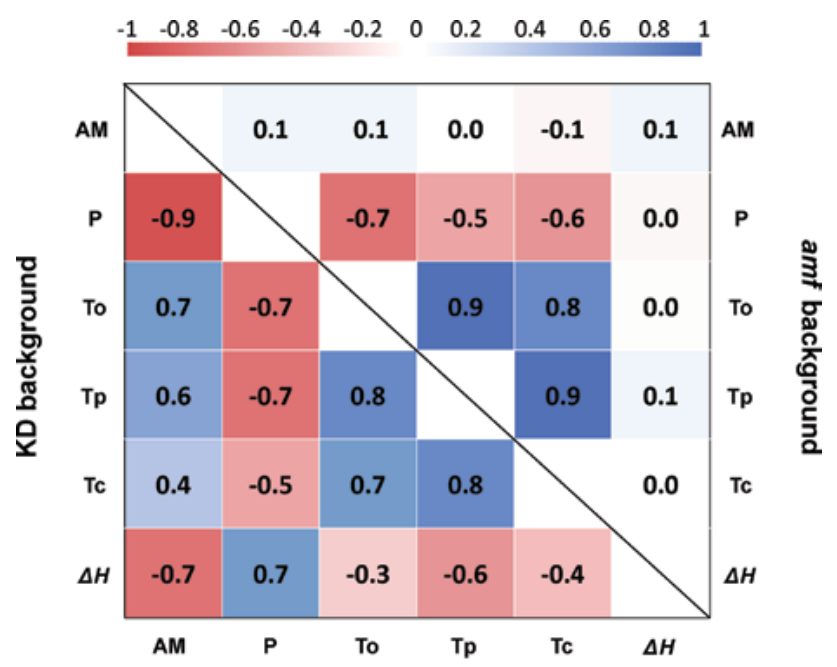

FIGURE 6. Heat-map displaying the extent and direction of correlations $(r)$ between starch compositional characters and starch physico-chemical properties in both KD and amf backgrounds. Correlations were statistically significant at $r>0.36$ and $r<-0.36$. Blue colours show negative correlations and red colours show positive correlations. AM, amylose content; $\mathrm{P}$, phosphate content; $\mathrm{T}_{\mathrm{o}^{\prime}} \mathrm{T}_{\mathrm{p}}$ and $\mathrm{T}_{\mathrm{c}^{\prime}}$ onset, peak and conclusion temperature of gelatinization; $\Delta H$, gelatinization enthalpy.

To examine the effect of phosphate content on freeze-thaw stability of starch gels, starches with different levels of phosphate content (listed in Table 1) were selected and the syneresis of a $5 \%$ starch gel after each of the four freezing and thawing cycles (Fig. 7) was measured. Low-phosphate starches (KDGl-5 and -14) showed dramatic syneresis (more than 70\%) after the first cycle and reached nearly to the maximum level. KDGl1 and KDGI-25 starches exhibited intermediate freeze-thaw stability in the first two cycles, which correlates with the medium level of phosphate content. High-phosphate starches (KDGII-6 and -17) and UT-KD displayed much better stability in the first two cycles compared to other lines, but did not show differences after 4 cycles. Similar patterns were observed in the freeze-thaw stability of starch gels from amf background. The syneresis of low-phosphate starches (amfGl-9 and amfGl-12) was $60 \%$ after the first cycle and raised to $80 \%$ with additional cycles, while UT-amf and medium/high-phosphate starches (amfGll-16 and -18) showed a lower syneresis ( 10\%) after the first cycle.

Starch digestibility was determined by measuring the amount of glucose after a-amylase and amyloglucosidase treatment. No significant changes were found between starches with different phosphate content in both backgrounds (data not shown). 
(a)

KD background

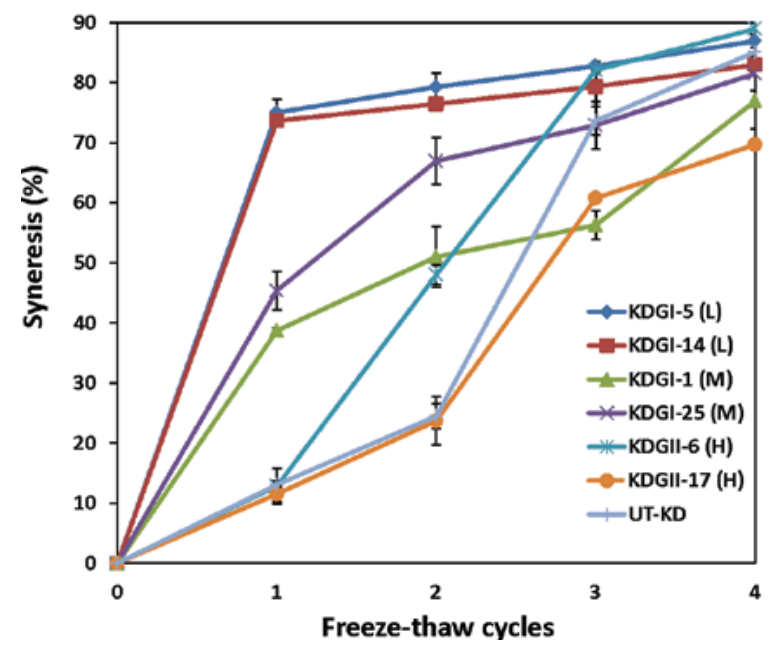

(b)

amf background

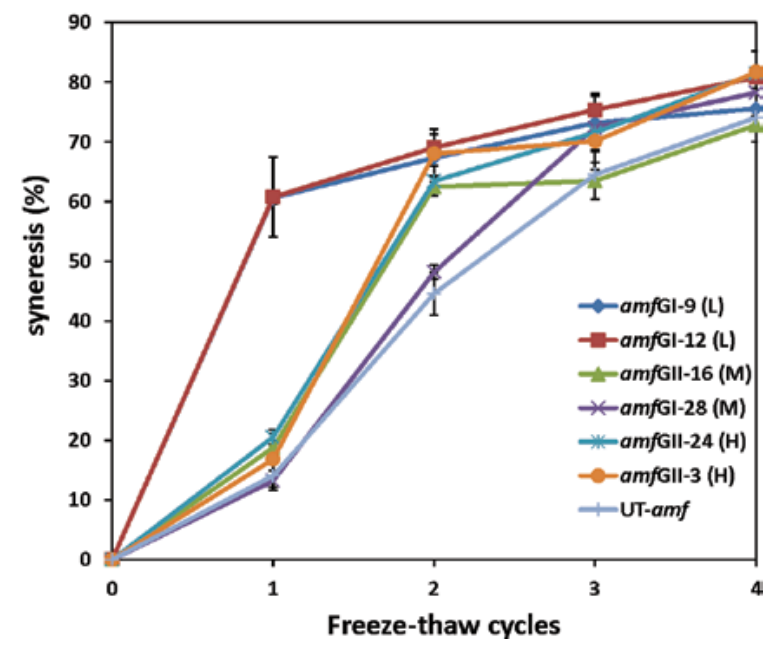

FIGURE 7. Freeze-thaw stability of starches from (a) KD and (b) amf background. The percentage of syneresis (the amount of water as a proportion of the initial sample weight) is shown at each cycle of freezethaw. L, M and $\mathrm{H}$ stand for low-, medium- and high-phosphate content in starches. The measurements are performed in triplicates and data are presented as mean \pm S.D. 
The expression of genes involved in starch metabolism and sugar-starch conversion is altered in KD transformants

To gain better insights into the effect of GWD1 expression on starch granule formation and starch metabolism, transformants with low and high GWD1 expression from each genetic background were subjected to RNA-seq analysis. Based on RNA-seq results, a range of differential expressed genes involved in starch metabolic pathways were selected and further investigated on more transformants using qRT-PCR analyses. We choose twelve transformants from each background, including six transformants with low-phosphate starches and six transformants with high-phosphate starches to examine the expression level of selected genes. Interestingly, the results showed that modulation of GWD1 expression affected the expression of various genes in the KD background, including $S P$, SSII, SSIII, SBEII, SUSY4, AGPase, GPT2 and INV, but did not influence the expression of GPT1, GBSSI, FK, AMY1, AMY2 and BAM. Changes in expression of all examined genes in amf transformants relative to control plants were much smaller than in KD background and not statistically significant (data not shown).

As shown in Fig. 8a, SUSY4 was strongly downregulated in KDGI transformants, whereas KDGIl transformants showed up to 3-fold increase in SUSY4 expression relative to the control. The expression of GPT2 in KDGl transformants decreased to $40 \%$ of that in the control line, while it increased up to 12-fold in KDGll transformants. Likewise, the expression level of INV decreased at least by half in KDGI transformants compared to the control, whereas the expression increased 11-fold in KDGIl transformants. Moreover, the expression of SBEII, SSII and SSIII exhibited a significant decrease in KDGI transformants relative to control. Taken together, modulation of GWD1 expression in potato tubers affects the expression of key genes participating in starch metabolism and sugar-starch conversion (Fig. 8b). 
(a)

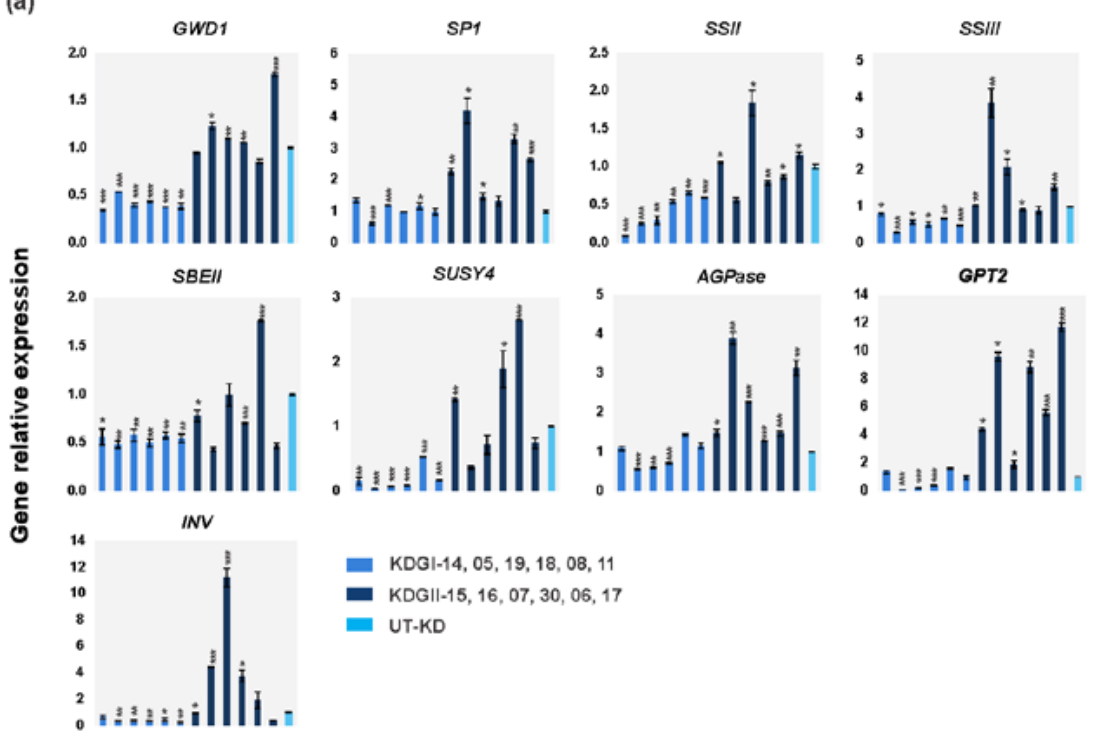

(b)

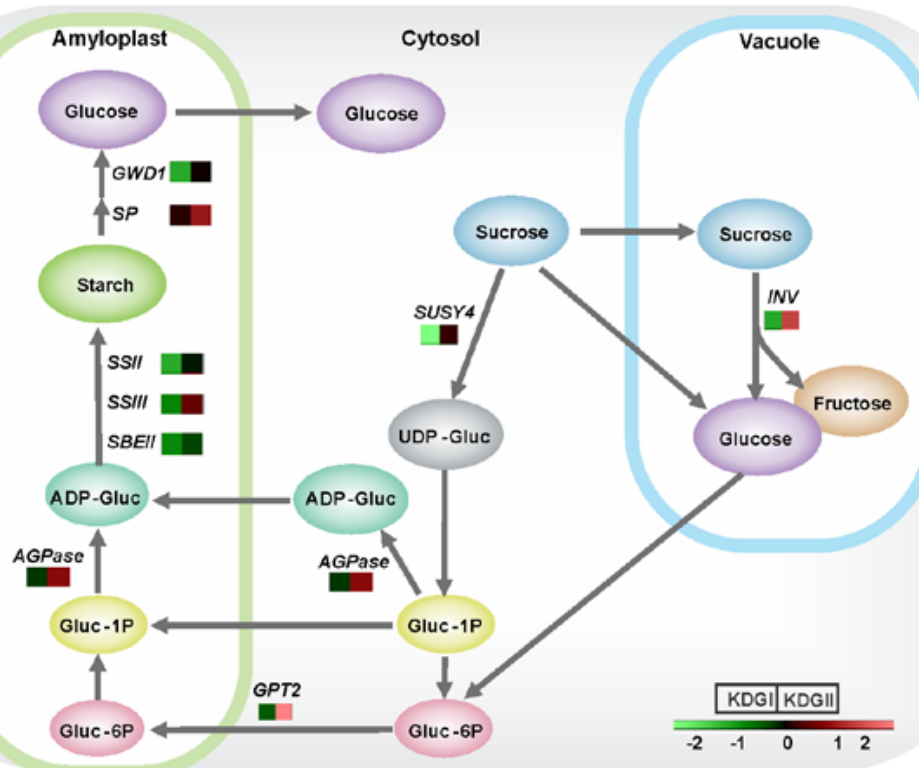

FIGURE 8. Effects of GWD1 expression on starch metabolism in the KD background. (a) Expression of key genes involved in starch metabolism in tubers. Include: starch synthases (SSII and SSIII), starch branching gene (SBEII), ADPglucose pyrophosphorylase (AGPase), Glc6P/phosphate translocator (GPT2), sucrose synthase 4 (SUSY4), invertase (INV) and starch phosphorylase (SP). Six transformants of each group, KDGI and KDGll, were selected and subjected to qRT-PCR analysis. Columns are arranged in ascending order of starch phosphate content of transformants. The values are expressed as the mean \pm SD. Significant differences were determined by using t-test ${ }^{*}, p<0.05 ; * *, p<0.01$ and ***, $p<0.001$ ). (b) Schematic illustration of starch metabolism in potato tubers. The average gene expression in transgenic tubers from KDGI and KDGII group is shown as false-colour box in the pathway, respectively. The values in red and green indicate log2 fold change in the average gene expression. 


\section{Discussion}

This study was initiated to increase the phosphate content of potato starch by overexpressing GWD1. Interestingly, we observed a broad range of phosphate contents in the starches of the transformants. Further analyses revealed a positive correlation between starch phosphate content and GWD1 expression levels, showing that the variation in starch phosphate content was a direct consequence of changes in GWD1 expression. The drastic decrease in GWD1 expression is likely due to the co-suppression of the transgene and endogenous GWD1 gene, based on the fact that overexpression of an endogenous gene may cause co-suppression, resulting in a null phenotype, as was shown originally with petunia flowers colour gene (Napoli et al., 1990; van der Krol et al., 1990). It has been reported that overexpression of granule-bound starch synthase (GBSS) resulted in co-suppression of this gene in potato transformants (Flipse et al., 1996b).

\section{Phosphate esters may act as identifiers of amylose and amylopectin}

Granule morphology analyses showed severely fissured granules in KD starches containing low phosphate content, indicating that the organization of the granule matrix was disrupted. Similar morphological disturbances were observed in starches obtained from lines with suppressed soluble starch synthase (Craig et al., 1998; Edwards et al., 1999; Fulton et al., 2002; Lloyd et al., 1999). These authors suggested that the observed fractures might be caused by direct amylose-amylopectin interactions, which lead ultimately to disruption of the granule matrix. In this study, fissures occurred in KD starch granules with extremely low phosphate content (less than $10 \mathrm{nmol} / \mathrm{mg}$ starch), while fissures were eliminated in starch granules with phosphate content higher than $\sim 15 \mathrm{nmol} / \mathrm{mg}$ starch. These results imply that a sufficient amount of phosphate content is important for a normal granule packing in the amylose-containing potato starches and the minimal amount could be $15 \mathrm{nmol}$ phosphate /mg starch. On the other hand, no fissured granules were observed in the amf starches regardless of the amount of starch phosphate content. Taken together, we propose that a sufficient amount of phosphate esters existed in amylopectin which may act as identifiers to distinguish amylopectin chains from amylose chains, which prevents the erroneous interactions between amylose and amylopectin segments and keeps the normal assembly of starch granules. This model confirms the hypothesis proposed by Blennow et al. (2002 \& 2003).

\section{Starch phosphorylation affects starch synthesis in potato tuber}

It has been reported that starch is in a continual state of carbon flux during tuber development, which is continuously both synthesized and degraded, thus the net gain or loss of starch is a result of the balance between synthetic and degradative enzymes 
(Isherwoo, 1973; Lewis et al., 1994). In this study, one would expect the increase in starch content of mature tubers with suppressed GWD1, as GWD1 was proven to participate in starch degradation. However, no significant effect on starch content was detected. This implies that the reduction in starch degradation might be accompanied by a reduction in starch synthesis, leading ultimately to no changes in starch content of tubers. A previous study on Arabidopsis has also demonstrated that the reduction of starch degradation by GWD1 suppression inhibited starch synthesis in leaves (Skeffington et al., 2014). Overall, we reason that starch phosphorylation might stimulate not only starch degradation but also starch synthesis in potato tubers.

Our view is further supported by the observation that various genes involved in pathways of starch synthesis and sucrose-starch conversion were down regulated in lines with suppressed GWD1. For instance, SUSY4 expression was strongly down-regulated in these lines, indicating a reduction of sink strength in tubers. Correspondingly, GPT2 expression was also down-regulated, suggesting a limited supply of Gluc-6P from cytosol to amyloplast. These alterations could conceivably lead to a decrease in flux from cytosol to amyloplast for starch synthesis, and this might also be the reason for the suppression of starch-synthesizing genes such as SBEII, SSII and SSIII. Collectively, we propose that starch phosphorylation regulates starch synthesis by controlling the carbon flux into starch while simultaneously modulating starch-synthesizing genes.

\section{Effects of phosphate on starch composition and properties}

Through the exhaustive characterization of transgenic series from two genetic backgrounds, strong correlations between phosphate content and starch compositional characters, such as amylose content and starch fine structure have been established. These results demonstrate the crucial role of GWD1 in starch granule formation and starch synthesis, which further supplements the findings of Vikso-Nielsen et al. (2001) and substantiates the effects of phosphate content on amylose content and starch structure. These alterations could be a combined effect of the changes in SBEII, SSII and SSIII expression. In previous studies it has been shown that the inhibition of these genes either individually or in combinations result in changes in amylose content and chain length distribution of amylopectin (Abel et al., 1996; Edwards et al., 1999; Edwards et al., 1995; Jobling et al., 1999; Lloyd et al., 1999; Marshall et al., 1996). On the other hand, altered GWD1 expression levels did not influence the distribution of glucan chains on the granule surfaces. This result differs from the observation obtained in transitory starches, showing that short glucan chain residues are located at the granule surface in Arabidopsis thaliana with reduced GWD1 activity (Mahlow et al., 2014). This suggests that GWD1 might play different roles in transitory starch and storage starch during starch biosynthesis. 
Negative correlations were observed between starch phosphate content and gelatinization temperatures in both backgrounds (Table 1, Fig. 6), indicating that lack of phosphate content leads to starch with a higher crystallinity. This supports the molecular model proposed by Blennow and Engelsen (2010), who suggested that phosphate esters disrupt double helix conformation thereby preventing optimal crystalline packing. Moreover, no correlation between phosphate content and gelatinization enthalpy was observed in the amf background, indicating that phosphate esters do not affect gelatinization enthalpy of starch. The positive correlation between these two observed in the KD background is likely an indirect effect caused by the suboptimal crystalline structure resulting from the loss of phosphate content or/and alterations in starch fine structure.

The freeze-thaw stability of starch was found to correlate with the phosphate content in starch, as the syneresis of starch gels increases with a lower starch phosphate content, which is in line with previous studies showing that phosphate groups contribute to the freeze-thaw stability (Bindzus et al., 2002; Nierle, 1990; Swinkels, 1985; Waliszewski et al., 2003). No changes in starch digestibility was observed between starches from tranformants and control plants, which could be a combined effect of changes in starch phosphate content, granule surface characteristics, fine structure of amylopectin and amylose content. These factors have been proven to affect the susceptibility of starches to enzymatic breakdown, leading to the changes in starch digestibility (Dhital et al., 2010; Hoover, 2001; Noda et al., 2008; Singh et al., 2010). 


\section{Supplementary data}

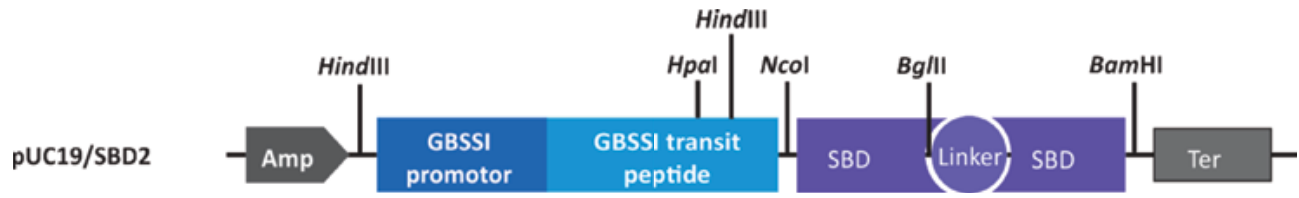

FIGURE S1. Schematic representation of pUC19/SBD2 vector. SBD, Linker, Amp and Ter stand for starch binding domain of cyclodextrin glycosyltransferase from B. circulans, an artificial PT-linker, ampicillin resistant gene and terminator, respectively. To generate this construct, a sequence encoding the potato GBSSI promoter and part of GBSSI transit peptide (HindIII - HindIII) was amplified from the construct pBIN19/SBD2 (Ji et al., 2004)

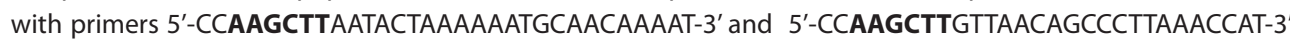
and inserted into the corresponding sites of the pUC19 vector. The orientation of the HindIII - Hindlll fragment was verified by sequencing. Subsequently, the sequence containing a SBD2 fragment and a part of GBSSI transit peptide (Hpal - BamHI) was amplified from pBIN19/SBD2 with primers 5'CGTTAACAAGCTTGATGGGCTCCAATCAAGAACT-3' and 5'-CGGGATCCGCCAAAACAGCCAAGCTTATG-3', followed by cloning into corresponding sites of pUC19.

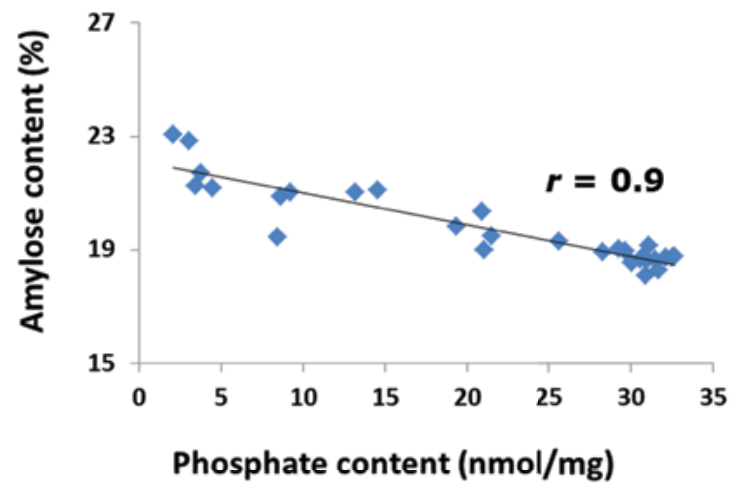

FIGURE S2. Plot of amylose content versus the phosphate content in starches from KD background. Correlation coefficient $(r)$ is indicated. 


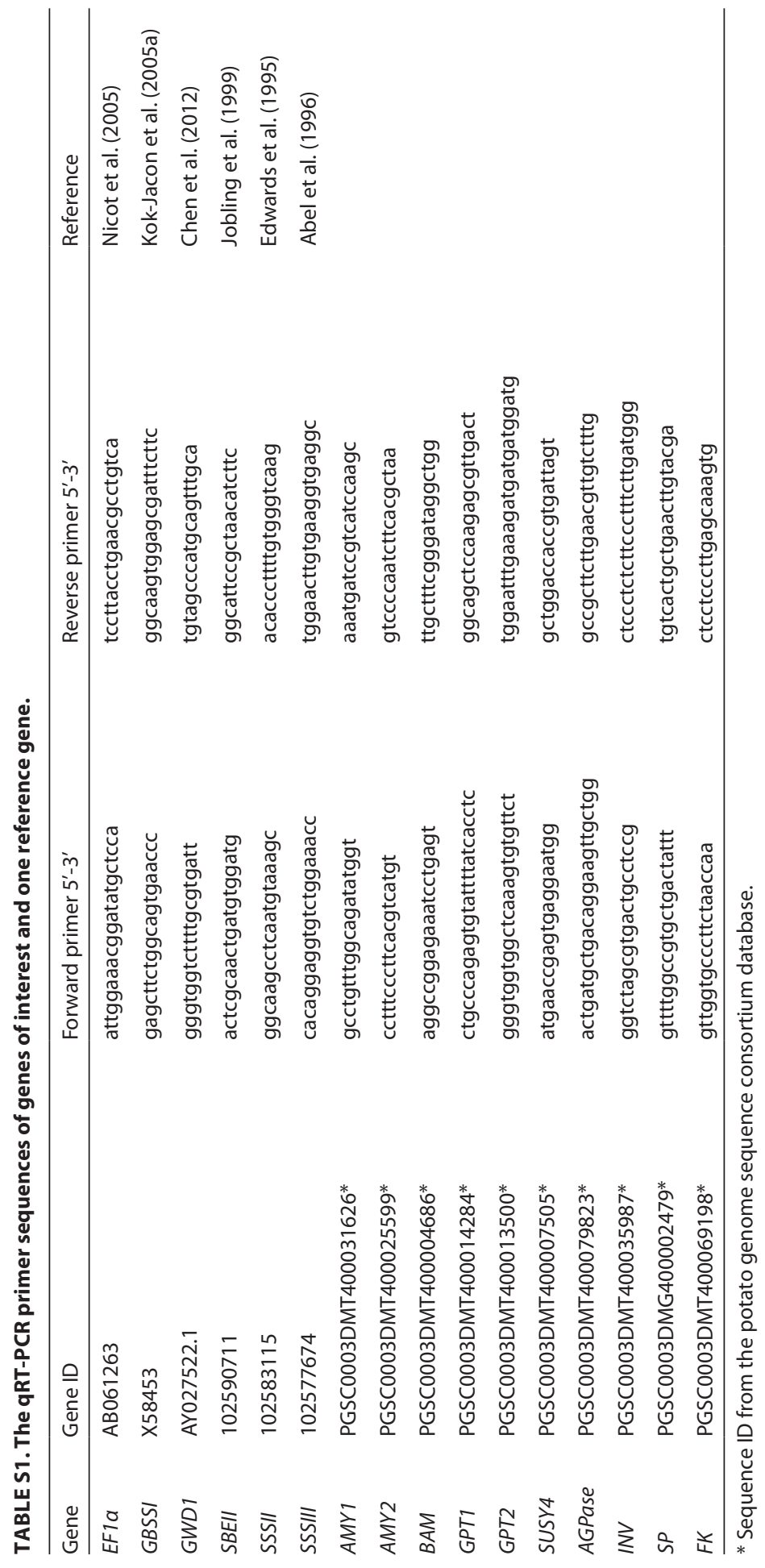





\title{
Chapter 5
}

\section{Introduction and Expression of Arabidopsis Starch Dikinases in Potato}

\author{
Xuan $\mathrm{Xu}^{1,2}$, \\ Dianka Dees ${ }^{1}$, \\ Xing-Feng Huang ${ }^{1,3}$, \\ Richard G.F. Visser ${ }^{1}$, \\ Luisa M. Trindade ${ }^{1}$
}

${ }^{1}$ Wageningen UR Plant Breeding, Wageningen University and Research, P.O. Box 386. 6700AJ Wageningen, The Netherlands.

${ }^{2}$ National Centre for Vegetable Improvement (Central China), Key Laboratory of Horticultural Plant Biology, Ministry of Education, Huazhong Agricultural University, Wuhan, 430070, China.

${ }^{3}$ Current address: Department of Chemical and Biological Engineering, Colorado State University, 1370 Campus Delivery, Fort Collins, CO 80523-1370, USA. 


\begin{abstract}
Starch phosphate esters influence physiochemical properties of starch granules that are essential both for starch metabolism and industrial use of starches. Potato starches have considerably higher phosphate content than cereal starches, providing improved functionalities for a wide range of applications. To modify properties of potato starch and understand the effect of starch phosphorylation on starch metabolism in storage starch, the starch dikinases from Arabidopsis thaliana, glucan water dikinase 2 and 3 (AtGWD2 and AtGWD3), were heterologous expressed in potato tubers from two genetic backgrounds: the amylose-containing clone Kardal and the amylose-free mutant amf. Modified starches showed altered granule morphology, but no significant changes in starch phosphate content were observed. Genes involved in starch metabolism did not show altered expression in the transgenic lines relatively to the control; however, sucrose synthase 4 (SUSY4) was upregulated in transgenic tubers with AtGWD2 expression. Taken together, these results demonstrated that expression of AtGWD2 and AtGWD3 are not suitable for engineering starch with a high phosphate content in storage organs where they are normally not expressed.
\end{abstract}




\section{Introduction}

Starch is the primary energy reserve in higher plants and the most important resource for animal and human energy intake. Starch granules are mainly composed of amylose ( $25 \%)$ and amylopectin ( $75 \%)$ regardless of botanic origin and synthesized in plastids as two main types, transitory and storage starch. Transitory starch is produced in chloroplasts during daytime photosynthesis and remobilized into sugars for respiration and growth at night (Zeeman et al., 2007b). Storage starch is accumulated in amyloplasts of heterotrophic organs (e.g. potato tubers, cereal seeds and cassava roots) and when required by the plant, hydrolysed to support phases of growth (Xu et al., 2014).

In the past decades, substantial progress has been made to elucidate the metabolism of transitory starch by using the model plant Arabidopsis thaliana. A breakthrough came with the discovery of the essential role of starch phosphorylation in starch metabolism. It has been shown that phosphate groups are built into the starch granules during starch biosynthesis for an efficient and accurate granule packing and physical amorphisation prior to degradation (Blennow, 2015). The presence of phosphate groups leads to a local structural disturbance within the starch granule and by that facilitates the access of degrading enzymes and promote starch degradation. Phosphate groups are found attached to amylopectin chains at both C-6 ( 60\%) and C-3 ( 30\%) positions of the glucose residue (Baysmidt et al., 1994; Hizukuri et al., 1970). Two enzymes are responsible for this process, namely, glucan, water dikinase (GWD1) and phosphoglucan, water dikinase (GWD3).

GWD1 was proven to phosphorylate glucose residues exclusively at the C-6 position (Ritte et al., 2006), while GWD3 phosphorylate glucose residues exclusively at the C-3 position with substrate specificity requirements for pre-phosphorylated starch (Baunsgaard et al., 2005; Kotting et al., 2005). GWD3 was first discovered in Arabidopsis thaliana and named AtGWD3. Very recently, GWD3 was also isolated and identified in potato, and named StGWD3. This gene has $58.6 \%$ identity and $75.3 \%$ similarity to AtGWD3 (Fig. 1, (Orzechowski et al., 2013). Additionally, a third glucan, water dikinase termed AtGWD2 was also first discovered in Arabidopsis thaliana and identified as an active cytosolic isoform of GWD1 due to the absence of transit peptide (Fig. 1, (Glaring et al., 2007). This gene is able to phosphorylate a-glucans in vitro and does not require pre-phosphorylation of substrate. Analysis of mutants suggested that AtGWD2 is not directly involved in transitory starch degradation. So far, only GWD1 has been extensively studied in various crops (e.g. rice, maize, wheat, barley and potato), where much attention has been focused on the manipulation of gene expression or expression of orthologous gene to modify phosphate content in starch granules or investigate the effect of starch phosphorylation in plant primary metabolism (Carciofi et al., 2011; Lanahan and Basu, 2004; Lorberth et al., 1998; Schewe et al., 2002). 
The importance of starch phosphorylation is not limited to the relevance of starch metabolism in plant survival, but also due to its positive impact on the starch functionality. Unlike cereal starch, potato starch contains high phosphate content conferring a high swelling capacity to starch gels, which is desired in many industrial processes (Jobling, 2004; Vikso-Nielsen et al., 2001). In industry, starch is often chemically or physically modified to gain such properties; however, these modifications are expensive and frequently produce hazardous waste. Therefore producing starches with enhanced properties in planta is needed to fully meet urgent demands for environmentally friendly starch production. To achieve this, a comprehensive understanding of starch phosphorylation is fundamental. To date, however, the role and molecular mechanism of starch phosphorylation are unclear in commercially important storage starches.

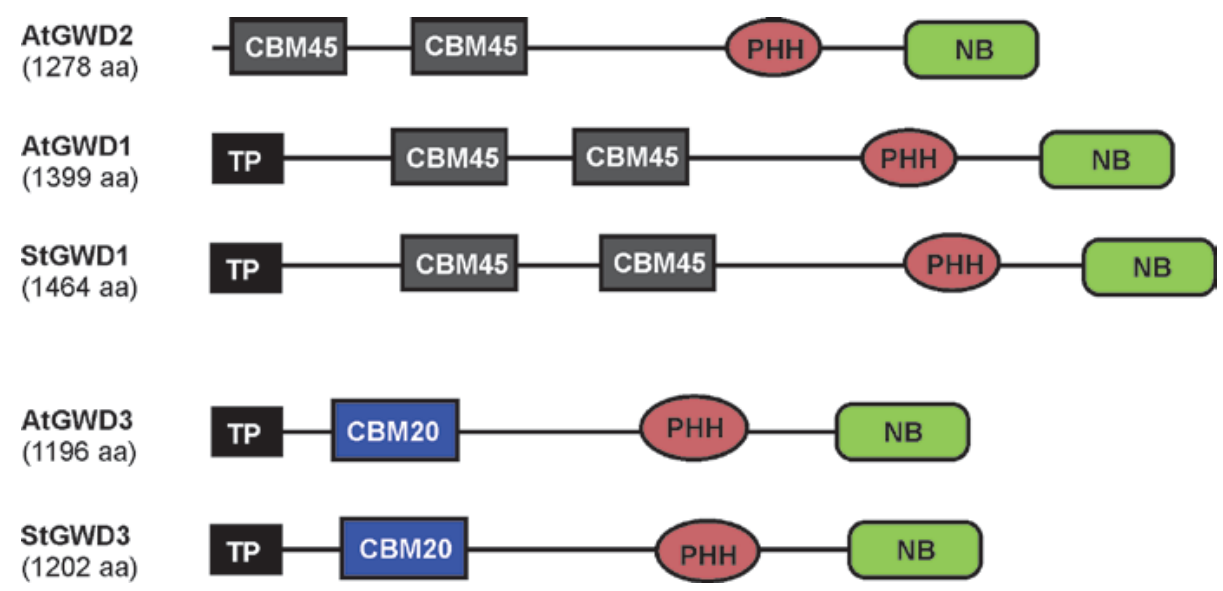

FIGURE 1. Schematic comparison of the domain structure of GWDs from Arabidopsis thaliana (At) and potato (St). TP, PHH and NB stand for transit peptide, phosphohistidine domain and nucleotide binding domain, respectively.

To explore the mechanism of starch phosphorylation in storage starch and produce starch with altered properties in planta, starch phosphorylating enzymes from Arabidopsis thaliana glucan water dikinase 2 and 3 (AtGWD2 and AtGWD3) were introduced into two potato genetic backgrounds, Kardal and amf mutants, respectively. The effects on starch phosphate content, granule morphology, properties and starch metabolism pathway have been examined and are discussed. 


\section{Materials and methods}

\section{Phylogenetic analysis}

Phylogenetic analysis was performed according to the procedure used by Baunsgaard et al. (2005); Glaring et al. (2007) with modifications. The tree was expanded by including all available GWDs sequences from GenBank. The alignment of sequences was created using MUSCLE method in MEGA6 based on the nucleotide binding domain of AtGWD3 (amino acids 1006-1194), and then submitted to the Minimum Evolution method (Rzhetsky and Nei, 1992) to generate the tree. The bootstrap consensus tree inferred from 1000 replicates was chosen to represent the evolutionary history of GWDs. GenBank accession numbers of each sequence are provided in Table 1.

TABLE 1. GenBank accession numbers of GWDs sequence used in phylogenetic analysis.

\begin{tabular}{|c|c|c|c|}
\hline \multirow{2}{*}{ Species } & GWD1 & GWD2 & GWD3 \\
\hline & \multicolumn{3}{|l|}{ GenBank accession no. } \\
\hline Arabidopsis thaliana (At) & NP563877 & AAO42141 & AY747068 \\
\hline Arabis alpina & & & KFK26410.1 \\
\hline Brachypodium distachyon & KQJ94575.1 & KQK18354.1 & KQK19799.1 \\
\hline Brassica napus & & & XP_013737454.1 \\
\hline Brassica oleracea var. oleracea & & & XP_013608682.1 \\
\hline Brassica rapa & & & XP_009111699.1 \\
\hline Camelina sativa & & & XP_010493901.1 \\
\hline Capsella rubella & & & EOA19824.1 \\
\hline Chlamydomonas reinhardtii & XP_001700833.1 & XP_001690609.1* & XP_001697099.1 \\
\hline Citrus reticulata & AAM18228 & & \\
\hline Citrus sinensis & XP_006483916.1 & XP_006468769.1 & XP_006493516.1 \\
\hline Cryptosporidium parvum & XP_626438.1 & & \\
\hline Eutrema salsugineum & & & ESQ32164.1* \\
\hline Glycine max & XP_006602393.1 & XP_006578296.1* & XP_006589801.2 \\
\hline Gossypium arboreum & & & KHF99077.1 \\
\hline Gossypium raimondii & & & XP_012454921.1 \\
\hline Hordeum vulgare & CAX51385.1 & CAX51386.1* & CAX51387.1* \\
\hline Kalanchoe fedtschenkoi & AIR93787.1 & & \\
\hline Lysopersicon esculentum & ACG69788.1 & & \\
\hline Manihot esculenta & AF083529.1 & AFO83530.1 & AF083531.1 \\
\hline Medicago sativa & CAE84830 & & \\
\hline Medicago truncatula & & & XP_013453267.1 \\
\hline Micromonas pusilla & XP_003058487.1 & & \\
\hline Micromonas sp. & XP_002509308.1 & & \\
\hline Nelumbo nucifera & BAK09334.1* & & \\
\hline Neospora caninum & CEL69439.1 & & \\
\hline Ostreococcus tauri & XP_003080650.1* & & XP_003080699.1* \\
\hline Oryza sativa & AK103463 & & XP_015620009.1 \\
\hline Physcomitrella patens & & & EDQ82900.1 \\
\hline Populus euphratica & XP_011045278.1 & XP_011016992.1 & XP_011000022.1 \\
\hline
\end{tabular}




\begin{tabular}{|c|c|c|c|}
\hline \multirow{2}{*}{ Species } & GWD1 & GWD2 & GWD3 \\
\hline & \multicolumn{3}{|c|}{ GenBank accession no. } \\
\hline Solanum tuberosum (St) & Q9AWA5 & & ACZ66259.1 \\
\hline Tarenaya hassleriana & & & XP_010546804.1 \\
\hline Theobroma cacao & & & EOY05043.1 \\
\hline Toxoplasma gondii & CEL77021.1 & & \\
\hline Triticum aestivum & CAC22583 & & \\
\hline Vitis vinifera & XP_010651715.1 & XP_010664437.1* & XP_002265211.1 \\
\hline Zea mays & AY109804* & & XP_008662597.1* \\
\hline
\end{tabular}

* Sequence was not included in tree construction due to the poor quality or lack of nucleotide binding domain.

\section{Plasmid construction}

Two constructs, pBIN19/GWD2 and pBIN19/GWD3, were made for ectopic expression of glucan, water dikinase 2 (AtGWD2, GenBank: AAO42141) and glucan, water dikinase 3 (AtGWD3, GenBank: AY747068) in potato, respectively. All constructs were fully sequenced to verify their correctness. The construct pBIN19/SBD previous reported (Ji et al., 2003) was modified by adding an Xbal site to generate pBIN19/SBD-Xbal in order to clone each of the two GWD genes (Supplementary Fig. S1).

For the assembly of the pBIN19/GWD2 construct, the AtGWD2 encoding fragment was generated by PCR amplification with the primers 5 '- TGCTCTAGAGCA GACTACAAAGACGATGACGATAAAACTAGTATGGCAACCTCTAAAT-3' (FLAG-encoding sequence underlined) and 5'- GCGTCGACGTTCAGTGGTGGTGGTGGTGGTGCTCGAGAACTTGGGGTCTAGCT-3' (HIS-encoding sequence underlined), which contained Xbal and Sall sites at $5^{\prime}$ ends, respectively (in bold). This amplified fragment, containing an N-terminal FLAG tag, AtGWD2 and a C-terminal HIS tag, was cloned into the same restriction sites of pBIN19/ SBD-Xbal, generating the pBIN19/GWD2 plasmid. Construct pBIN19/GWD3 was obtained using the same procedure. A fragment containing FLAG tag, tGWD3 and a HIS tag was amplified by PCR amplification with the primers 5'- TGCTCTAGAGCAGACGATAAAACTAGTATGGAGAGCATTGGCAGCCATTGT-3' (FLAG-encoding sequence underlined) and 5'- GCGTCGACGT TCAGTGGTGGTGGTGGTGGTGCAGAGGTTGTGGCCTTGACTGAAC-3' (HISencoding sequence underlined) (Fig. 2).

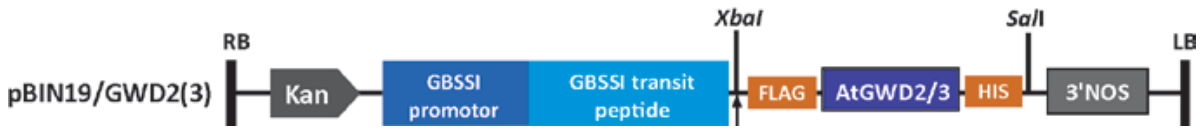

FIGURE 2. Schematic depiction of the constructs pBIN19/GWD2 and pBIN19/GWD3. AtGWD2 and AtGWD3 were cloned in frame with GBSSI transit peptide to allow amyloplast targeting and were driven by GBSSI promoter for tuber expression. RB and LB stand for right and left borders, respectively. Kan and 3'NOS stand for kanamycin resistant gene and NOS terminator, respectively. The arrow represents cleavage site of the transit peptide. FLAG and HIS are two tags for protein quantification and Xbal and Sall are restriction enzymes. 


\section{Transformation and regeneration}

Constructs were introduced into both amylose-containing (Kardal) and amylose-free (amf) potato genetic backgrounds via Agrobacterium. Four transgenic series KDG2, KDG3, amfG2 and amfG3 were yielded in which G2, G3, KD and amf represent AtGWD2, AtGWD3, Kardal and amf background, respectively. Thirty independent transgenic plants of each series as well as control plants (untransformed plants and transformed plants with empty vector) were multiplied to five plants and grown in the greenhouse under standard conditions, $16 \mathrm{~h}$ light at $20^{\circ} \mathrm{C}$ and $8 \mathrm{~h}$ dark at $18^{\circ} \mathrm{C}$. Mature tubers were harvested at the end of the life-cycle. No differences were detected between untransformed lines and transformed plantlets with empty vector, therefore they were further referred to as control or UT.

\section{Starch isolation}

Tubers from five plants from the same clone were pooled together to minimize individual variation, and starch isolation was performed according to the procedure described in Huang et al. (2013).

\section{Analysis of starch characteristics}

All analyses conducted for starch characteristics have been performed in duplicate unless indicated otherwise.

Starch chain length distribution (CLD) was determined by using high-performance anionexchange chromatography with pulsed amperometric detection (HPAEC-PAC) according to the method of Huang et al. (2013).

Particle size distribution was measured with a Coulter Multisizer II (Beckman-Coulter, UK) equipped with a 200- $\mu \mathrm{m}$ aperture tube. Ten $\mathrm{mg}$ of starch samples were suspended in a particle-free electrolyte (ISOTON ${ }^{\circledR}$ II, Beckman-Coulter, UK), and then analysed by counting 50,000 particles with coincidence factor set at $10 \%$. Significant differences in particle size were assessed by one-way general analysis of variance (one-way ANOVA) using GenStat (16th edition). The least significant difference values were calculated at $5 \%$ probability.

Starches were stained with $20 \times$ diluted Lugol's solution $\left(1 \% \mathrm{I}_{2} / \mathrm{KI}\right)$ and then investigated with light microscopy (LM, Axiophot, Germany). The detailed morphology on the granule surface was examined using scanning electron microscopy (SEM) Phenom ${ }^{\mathrm{TM}}$ (FEl, The Netherlands). One mg of starch samples were evenly distributed on a carbon tabs (Agar Scientific, UK) and mounted onto $12.70 \mathrm{~mm}$ aluminium specimen stubs (Agar Scientific, UK), followed by coating with gold using a sputter coater (EMITECH K550X; Quorum Technologies, UK). All images were digitally recorded. 
Gelatinization properties of starches were analysed by Differential Scanning Calorimetry (DSC), with Perkin-Elmer Pyris 6 (Perkin-Elmer, The Netherlands). The starch samples were adjusted to $80 \%$ moisture content in a stainless-steel pan by adding an appropriate amount of water according to the dry matter content of the starch samples. The pan was hermetically sealed followed by equilibration of the starch samples and the water at room temperature overnight. The samples were scanned in the temperature range from $40^{\circ} \mathrm{C}$ to $100^{\circ} \mathrm{C}$ at a rate of $10^{\circ} \mathrm{C} / \mathrm{min}$, using an empty pan as the reference. The onset $\left(\mathrm{T}_{\mathrm{o}}\right)$, peak $\left(T_{p}\right)$ and conclusion $\left(T_{c}\right)$ temperature of gelatinization and the melting enthalpy $(\Delta H, J / g)$ were determined.

The apparent amylose content was performed according to the procedure described in Hovenkamp-Hermelink et al. (1988).

Total phosphate content in the starch was determined according to the method described by Morrison (1964) with some modifications. Twenty mg of dry starch were suspended in $250 \mu \mathrm{l}$ of $70 \%(\mathrm{w} / \mathrm{w}) \mathrm{HClO}_{4}$ and completely charred at $250{ }^{\circ} \mathrm{C}$ for $25 \mathrm{~min}$. The solution was clarified by adding $50 \mu \mathrm{l} 30 \%(\mathrm{w} / \mathrm{v}) \mathrm{H}_{2} \mathrm{O}_{2}$ and gently boiled for $2 \mathrm{~min}$. After cooling to the room temperature, water was added to a final volume of $2 \mathrm{ml}$ and $100 \mu \mathrm{l}$ of the sample was transferred into a 96 -well microtiter plate, followed by adding $200 \mu$ l of colour reagent $\left[0.75 \%(\mathrm{w} / \mathrm{v})\left(\mathrm{NH}_{4}\right)_{6} \mathrm{Mo}_{7} \mathrm{O}_{24} \cdot 4 \mathrm{H}_{2} \mathrm{O}, 3 \%(\mathrm{w} / \mathrm{v}) \mathrm{FeSO}_{4} \cdot 7 \mathrm{H}_{2} \mathrm{O}\right.$ and $0.75 \%(\mathrm{w} / \mathrm{v}) \mathrm{SDS}$ dissolved in $0.375 \mathrm{M} \mathrm{H}_{2} \mathrm{SO}_{4}$ ]. Absorbance at $750 \mathrm{~nm}$ was then measured in a Model 680 XR Microplate Reader (Bio-Rad, US), and compared to the absorption of a calibration curve to calculate the concentration in $\mathrm{nmol} \mathrm{PO}_{4} / \mathrm{mg}$ starch.

\section{Quantitative RT-PCR (qRT-PCR) analysis}

Total RNA was extracted from potato tuber samples according to Kuipers et al. (1994) and reverse transcribed using the iScript cDNA synthesis kit from BioRad. Transcription levels of all target genes were determined in triplicate using the comparative Ct method (Livak and Schmittgen, 2001) and expressed relative to elongation factor EF1a (Nicot et al., 2005). The gene specific primers used in this study are listed in Supplementary Table S1.

The expression level of AtGWD2 and AtGWD3 was determined for all transformants and control plants. The relative expression level of target genes was multiplied by a factor of $10^{6}$ and then converted to $\log 10$. The resulting value $(\mathrm{v})$ was used to divide tranformants to different categories: undetectable $(N, v=0)$, low $(L, 0<v<2)$, medium $(M, 2 \leq v<3)$ and high $(H, v \geq 3)$ expressors.

Five or six high-expressors from each series and a few randomly picked control lines were further subjected to qRT-PCR for investigating the expression of key genes involved in starch metabolism. These genes were sucrose synthase (SUSY4), potato glucan water 
dikinase (StGWD1), potato phosphoglucan water dikinase (StGWD3), starch phosphatase (SEX4, LSF1 and LSF2), granule-bound starch synthase I (GBSSI), starch synthase III (SSIII), ADP-glucose pyrophosphorylase (AGPase), UDP-glucose pyrophosphorylase (UGPase), $\beta$-amylase (BAM), starch branching genes (SBEI and SBEII) and starch phosphorylase (SP).

\section{Results}

\section{GWDs are found in a wide range of organisms}

As there has been a continuous release of genome and transcriptome sequences in the last decade, previous phylogenetic analyses of GWDs (Glaring et al., 2007) was updated with sequences newly retrieved from GenBank databases. An extensive search was performed and a total of 48 sequences were obtained to construct the phylogenetic tree. The current tree included six and thirteen additional orthologues of AtGWD1 and AtGWD3, respectively, compared with the previous result (Glaring et al., 2007). Contrary to the widespread occurrence of GWD1 and GWD3, only a total of eight putative orthologues of AtGWD2 were found in higher plants and algae. Four sequences were excluded from the analysis due to the poor quality or lack of nucleotide binding domain, while another four sequences were used to construct the phylogenetic tree including three newly identified genes from Brachypodium distachyon, Manihot esculenta and Populous euphratica.

The tree was constructed using the Minimum Evolution method based on the highly conserved C-terminal ATP-binding domain of GWDs protein sequences. As shown in Fig. 3, two main clades of GWDs could be identified. One clade constituted a cluster with AtGWD1 and orthologues from other higher plants, the apicomplexan parasites and green algae, while another clade comprised the AtGWD3 and putative orthologues. Four representatives of GWD2 formed a group closely related to the GWD1 sequences, whereas the one from Brachypodium distachyon was classified into the clade of GWD1 and shown to be more closely related to GWD1 from cereal grasses. 


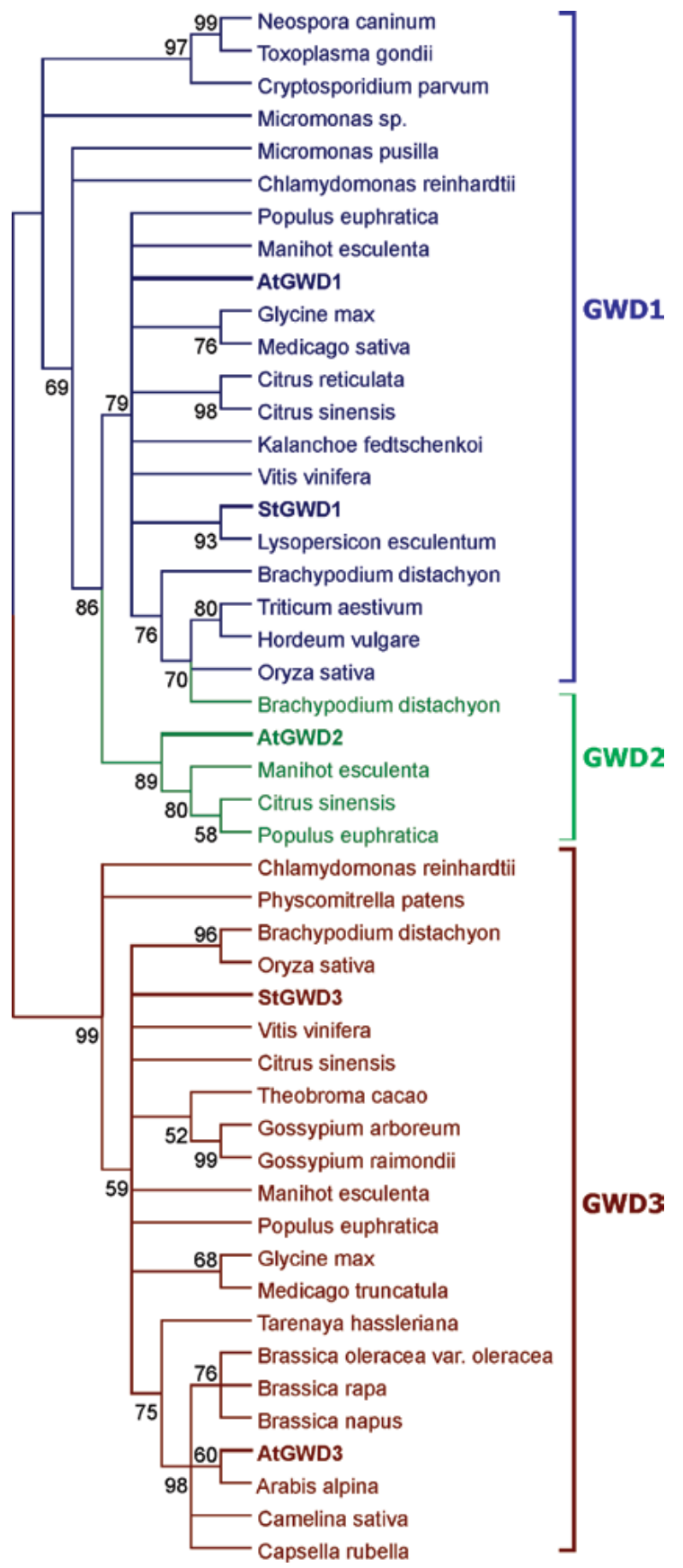

FIGURE 3. Bootstrap consensus tree ( 1000 replicates) of the nucleotide binding domain of GWD sequences from various organisms. Sequences were retrieved from GenBank and through BLAST, aligned and analysed in MEGA6. GWDs from Arabidopsis thaliana (AtGWD1, AtGWD2 and AtGWD3) and potato (StGWD1 and StGWD3) are indicated in bold. Bootstrap values greater than 50 are indicated at the branch points. Accession numbers are provided in Table 1. 


\section{Transformants show different expression levels}

Four transgenic series KDG2, KDG3, amfG2 and amfG3 were generated by introducing two constructs into potato genetic backgrounds, KD and amf. Thirty independent lines from each transgenic series and their respective controls were grown in the greenhouse until maturity. Plant architecture, tuber morphology and yield of transgenic lines were comparable to that of control plants (data not shown).

The expression of transgenes was analysed by qRT-PCR analyses. The transformants of each transgenic series were divided into 4 classes based on the expression level of each transgene: $\mathrm{N}, \mathrm{L}, \mathrm{M}$ and $\mathrm{H}$ represent undetectable (or none), low, medium and high expressors, respectively (Fig. 4). Generally, more transformants were classified as the Mand $\mathrm{H}$ - expressors regardless of constructs and backgrounds. To illustrate, $54 \%$ and $21 \%$ of KDG2 transformants exhibited medium and high expression, respectively, while the same amount of amfG2 transformants (42\%) were categorized as $\mathrm{M}$ - and $\mathrm{H}$-expressors. The $\mathrm{M}$ - and $\mathrm{H}$-expressors represented 30 and $48 \%$, respectively, of the KDG3 transformants, whereas the corresponding figures were 42 and $26 \%$ for amfG3 series. No L-expressors were observed in KDG3 or amfG2 series.

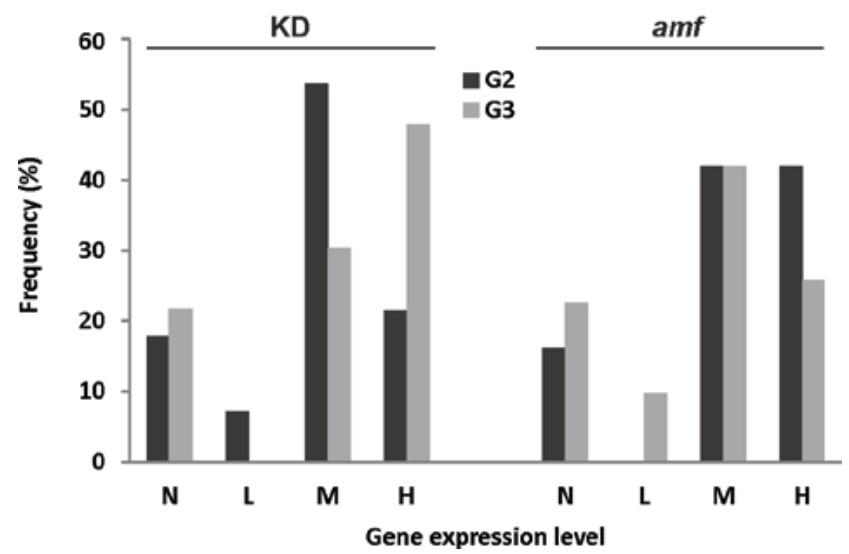

FIGURE 4. Distribution of the individual transformants over the classes of transgenes expression. $N, L, M$ and $\mathrm{H}$ stand for undetectable, low, medium and high expressors.

\section{Phosphate content is unaffected in modified starches}

Starch phosphate content was measured for all modified starches and their respective controls. As shown in Fig. 5, modified starches showed substantial variation in phosphate content. The phosphate content of KDG2 and KDG3 starches ranged, respectively, from 27 to $38 \mathrm{nmol} / \mathrm{mg}$ starch and 26 to $35 \mathrm{nmol} / \mathrm{mg}$ starch. Two starches from amfG2 transformants showed dramatically lower level of starch phosphate content $(<10 \mathrm{nmol} /$ $\mathrm{mg}$ starch) compared to the control starch ( $20 \mathrm{nmol} / \mathrm{mg}$ starch), which could be explained 
by the co-suppression of StGWD1. ANOVA analyses were performed to investigate the significance of changes in phosphate content and two starches with extremely low phosphate content from amfG2 series were excluded from these analyses. No significant changes in starch phosphate content from each series were found compared to the controls.

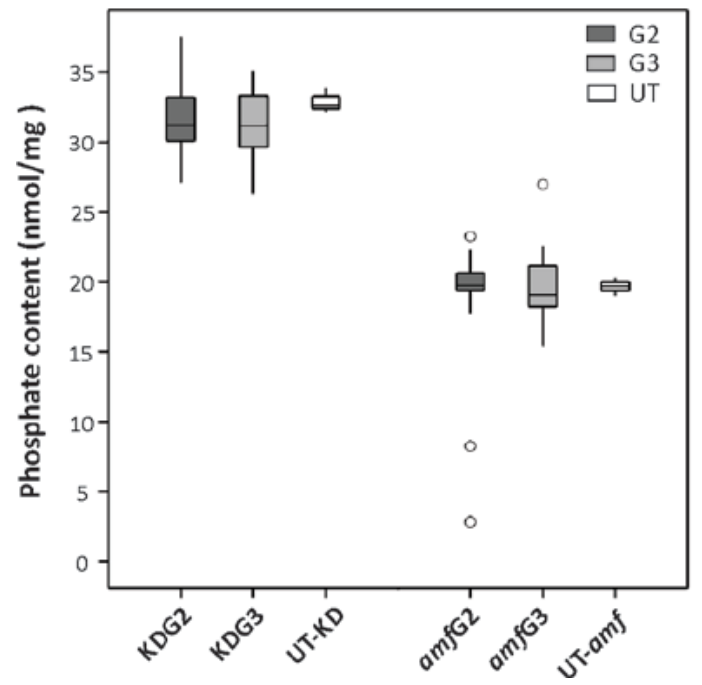

FIGURE 5. Box-plot representing the phosphate content of starches from all transformants in both genetic backgrounds and respective untransformed controls (UT-KD and UT-amf). Boxes in the plot include values in the $25 \%-75 \%$ interval, internal lines represent the median, black circles indicate outliers and bars represent extremes.

\section{Granule morphology and size are altered in modified starches}

To investigate whether the expression of AtGWD2 and AtGWD3 affects the morphology of starch granules, granule morphology was examined using light microscopy (LM) and electron scanning microscopy (SEM). Light microscopy analysis showed that the shape of starch granules from both KDG2 and KDG3 tranformants was irregular (Fig. 6D and G) in contrast to the regular oval shape observed in the UT-KD (Fig. 6A). Further analyses with SEM revealed that KDG2 and KDG3 starch granules have bumps on the surface (Fig. $6 \mathrm{E}$, $\mathrm{F}, \mathrm{H}$ and I), while the surface of starch granule from UT-KD was uniformly smooth (Fig. $6 \mathrm{~B}$ and $\mathrm{C})$. In the amf background, the control starch exhibited regular round-shaped granules (Fig. 6G-I). Starch granules from amfG2 transformants displayed helical shell structure occurring mostly with cracks and craters (Fig. 6J-L), whereas those from amfG3 transformants showed an elongated shape with craters on the surface of the granule (Fig. 6P-R). 


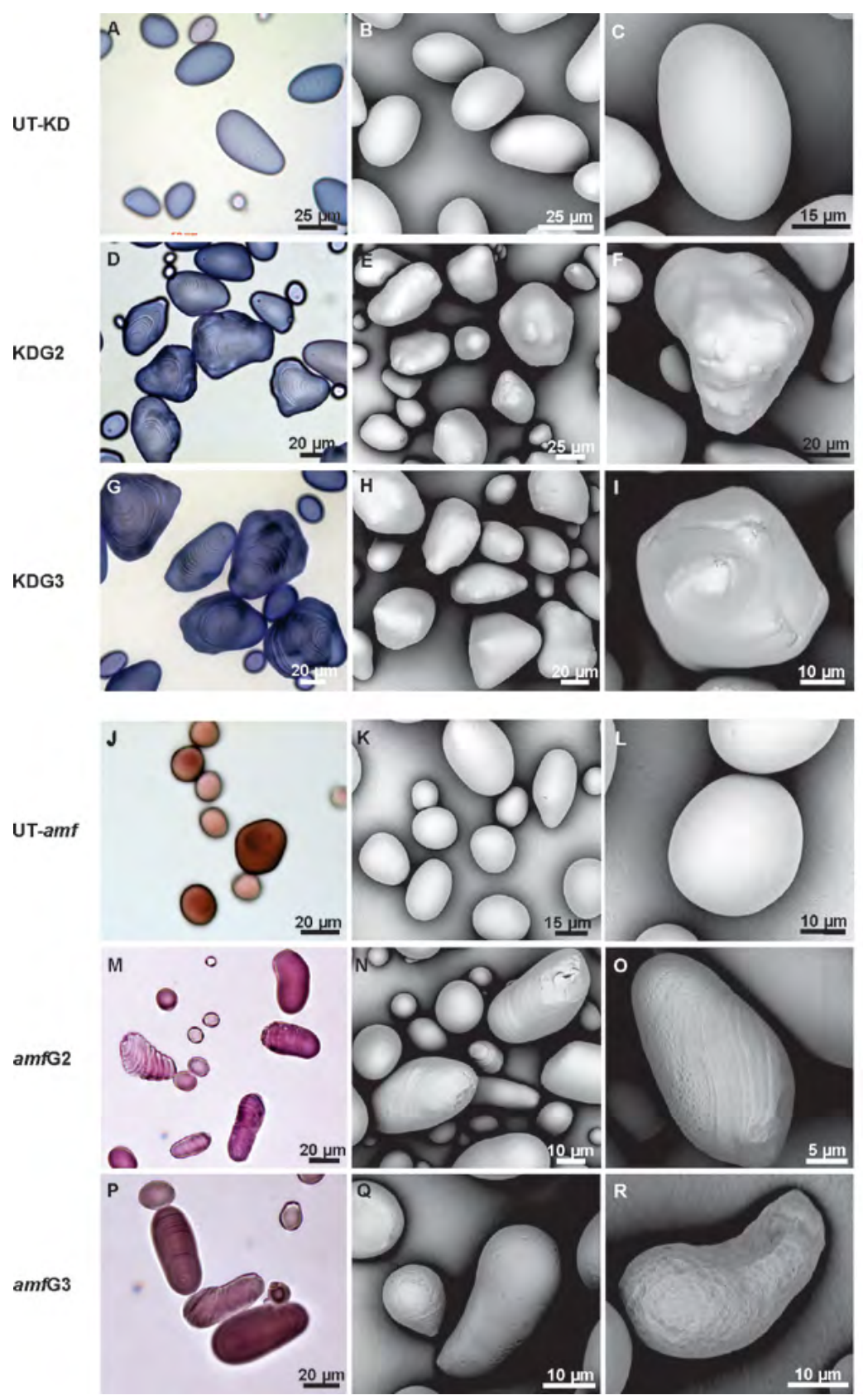

FIGURE 6. Granule morphology of starches from transformants and controls. Light microscopy (LM) and scanning electron microscopy (SEM) of starch granule morphology from UT-KD ( $A, B$ and C), KDG2 (D, E and F), $\operatorname{KDG} 3(\mathrm{G}, \mathrm{H}$ and I), UT-amf (J, K and $\mathrm{L}), \operatorname{amfG} 2(\mathrm{M}, \mathrm{N}$ and $\mathrm{O})$ and $\operatorname{amfG} 3(\mathrm{P}, \mathrm{Q}$ and $\mathrm{R})$. Starch granules were stained with a 20x diluted Lugol solution for light microscopy. 
The number of altered granules was recorded by counting 100 starch granules in triplicate for selected transformants with different levels of transgene expression. Starch granules with altered morphology were observed for KD transformants at all levels of gene expression. About $70 \%$ and $47 \%$ of starch granules showed altered morphology in $\mathrm{H}$-expressors from KDG2 and KDG3, respectively, which was markedly higher than those observed in L- and M-expressors (Fig. 7a). In the amf background, altered granules from both amfG2 and amfG3 series were mainly found in the H-expressors at a frequency of $21 \%$ and $54 \%$, respectively (Fig. $7 a$ ).

Granule size distribution analysis was performed for all modified starches and their respective controls. The results showed that the median granule size of both KDG2 and KDG3 starches ranged from 18 to $26 \mu \mathrm{m}$, while that of control starches was about 19 $\mu \mathrm{m}$. Statistical analysis revealed that the granule size of KDG2 and KDG3 starches was significantly increased compared to that of control starch ( $p<0.05$, Fig. 7b). In amfG2 and amfG3 no significant change in the granule size was observed relative to the control (Fig. 7b).

(a)

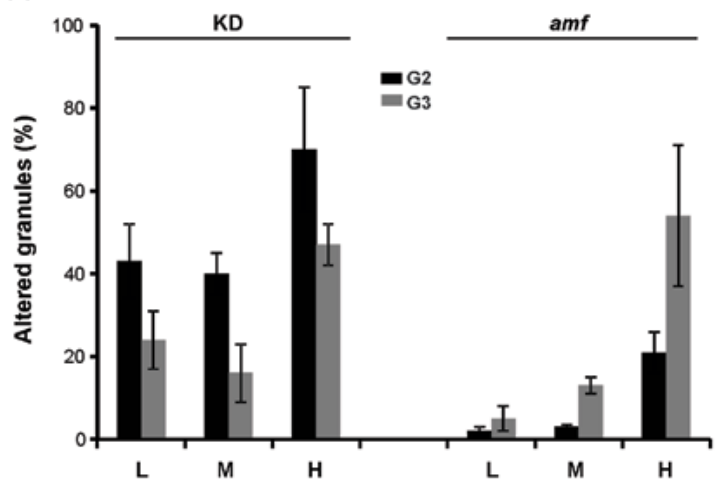

(b)

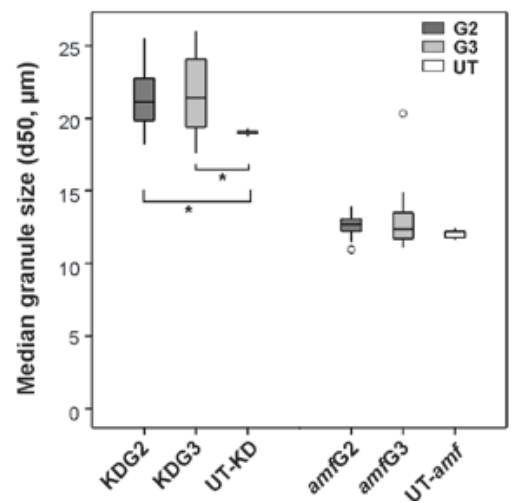

FIGURE 7. (a) Percentage of granules with altered morphology in transformants with different gene expression level: low (L), medium (M) and high (H). A population of 100 starch granules was counted in triplicate. The values are expressed as the mean \pm SD. (b) Boxplot presenting median granule size (d50) of modified starches and controls. Analyses were performed on all starches, which are 32, 32, 27 and 28 lines for KDG2, KDG3, amfG2 and amfG3 series, respectively. Boxes in the plot include values in the $25 \%-75 \%$ interval. Internal lines, unfilled circles and bars represent the median, outliers and extremes, respectively. Statistical significance was analysed using t-test $\left.{ }^{*}, p<0.05\right)$. 


\section{Neither starch fine structure nor properties were altered}

The chain length distribution of starches was determined after debranching starches with isoamylase by using HPAEC-PAC. No significant changes were detected in modified starches compared with the control starches in both backgrounds (data not shown). Moreover, amylose content and gelatinization properties did not show significant differences between modified starches and control starches (Table 2), indicating that expression of AtGWD2 and AtGWD3 does not influence these starch properties in neither KD nor amf background.

TABLE 2. Overview of characteristics determined for the representative starches in both backgrounds. Representative starches with different expression level in each series and respective control are presented.

\begin{tabular}{|c|c|c|c|c|c|c|c|}
\hline Clone & Class & $\mathrm{P}$ (nmol/mg) & AM (\%) & d50 ( $\mu \mathrm{m})$ & $\mathrm{T}_{0}\left({ }^{\circ} \mathrm{C}\right)$ & $\mathrm{T}_{\mathrm{c}}\left({ }^{\circ} \mathrm{C}\right)$ & $\Delta H(\mathrm{~J} / \mathrm{g})$ \\
\hline UT-KD & - & $32.8 \pm 0.8$ & $19.0 \pm 0.6$ & 19.1 & $66.5 \pm 0.3$ & $79.9 \pm 0.6$ & $19.6 \pm 1.3$ \\
\hline KDG2-24 & $\mathrm{N}$ & $32.8 \pm 0.2$ & $18.8 \pm 1.0$ & 21.0 & $66.7 \pm 0.2$ & $80.1 \pm 0.5$ & $18.8 \pm 1.2$ \\
\hline KDG2-25 & $\mathrm{L}$ & $33.7 \pm 0.1$ & $18.6 \pm 1.1$ & 24.4 & $66.8 \pm 0.5$ & $79.1 \pm 0.7$ & $18.3 \pm 0.3$ \\
\hline KDG2-15 & M & $29.4 \pm 0.4$ & $18.1 \pm 0.9$ & 21.4 & $65.8 \pm 0.2$ & $79.9 \pm 0.2$ & $20.0 \pm 0.1$ \\
\hline KDG2-23 & $\mathrm{H}$ & $30.9 \pm 0.3$ & $18.2 \pm 1.0$ & 20.0 & $67.0 \pm 0.0$ & $80.5 \pm 0.0$ & $18.4 \pm 0.4$ \\
\hline KDG3-28 & $\mathrm{N}$ & $33.8 \pm 0.4$ & $18.7 \pm 0.3$ & 22.0 & $66.5 \pm 0.1$ & $79.6 \pm 0.2$ & $17.7 \pm 0.0$ \\
\hline KDG3-25 & $M$ & $32.3 \pm 0.6$ & $18.6 \pm 0.3$ & 24.2 & $67.7 \pm 0.2$ & $81.6 \pm 0.7$ & $19.2 \pm 0.7$ \\
\hline KDG3-06 & $\mathrm{H}$ & $31.0 \pm 0.2$ & $18.5 \pm 0.1$ & 21.0 & $67.0 \pm 0.2$ & $80.0 \pm 0.5$ & $18.4 \pm 1.1$ \\
\hline UT-amf & - & $19.7 \pm 0.4$ & $3.2 \pm 0.4$ & 12.0 & $73.1 \pm 0.1$ & $84.7 \pm 0.1$ & $16.0 \pm 0.2$ \\
\hline$a m f G 2-02$ & $\mathrm{~N}$ & $19.2 \pm 0.1$ & $3.5 \pm 0.2$ & 13.1 & $72.7 \pm 0.3$ & $84.8 \pm 0.2$ & $15.8 \pm 0.4$ \\
\hline$a m f G 2-05$ & $M$ & $19.6 \pm 0.2$ & $4.2 \pm 0.4$ & 12.8 & $73.2 \pm 0.2$ & $86.1 \pm 0.1$ & $16.4 \pm 0.5$ \\
\hline$a m f G 2-23$ & $\mathrm{H}$ & $22.4 \pm 0.2$ & $3.7 \pm 0.0$ & 12.2 & $72.9 \pm 0.1$ & $86.4 \pm 0.3$ & $16.2 \pm 0.4$ \\
\hline$a m f G 3-08$ & $\mathrm{~N}$ & $20.5 \pm 0.1$ & $3.5 \pm 0.2$ & 11.1 & $73.7 \pm 0.5$ & $86.1 \pm 0.2$ & $16.2 \pm 0.1$ \\
\hline$a m f G 3-25$ & $\mathrm{~L}$ & $18.6 \pm 0.0$ & $3.2 \pm 0.5$ & 14.9 & $72.2 \pm 0.3$ & $85.4 \pm 0.1$ & $16.4 \pm 0.0$ \\
\hline amfG3-10 & $M$ & $19.0 \pm 0.3$ & $4.1 \pm 0.4$ & 11.5 & $72.9 \pm 0.0$ & $85.9 \pm 0.5$ & $15.7 \pm 0.4$ \\
\hline amfG3-24 & $\mathrm{H}$ & $21.0 \pm 0.0$ & $3.1 \pm 0.0$ & 11.7 & $73.8 \pm 0.1$ & $86.2 \pm 0.3$ & $16.4 \pm 0.2$ \\
\hline
\end{tabular}

Data (mean \pm S.D.) are the average of two or three independent measurements. $P$, total phosphate content; AM, apparent amylose content; $\mathrm{d} 50$, median granule size; $\mathrm{T}_{0}$ and $\mathrm{T}_{\mathrm{c}^{\prime}}$ starch gelatinization temperature; $\Delta H$, gelatinization enthalpy.

\section{SUSY4 was up-regulated in transformants carrying the AtGWD2 gene}

To investigate whether expression of AtGWD2 and AtGWD3 affects the expression of key genes involved in the starch metabolism, qRT-PCR analysis was performed with the highexpressors and a few random lines from each transgenic series. The results showed that the expression of SUSY4 was substantially upregulated in AtGWD2 transgenic tubers in both backgrounds (Fig. 8a and b), whereas no significant differences were found in the expression level of StGWD1, StGWD3, LSF1, LSF2, SEX4, GBSSI, SSIII, AGPase, UGPase, BAM, $S B E I, S B E I l$ and SP regardless of constructs and backgrounds (Fig. 8a-d). 

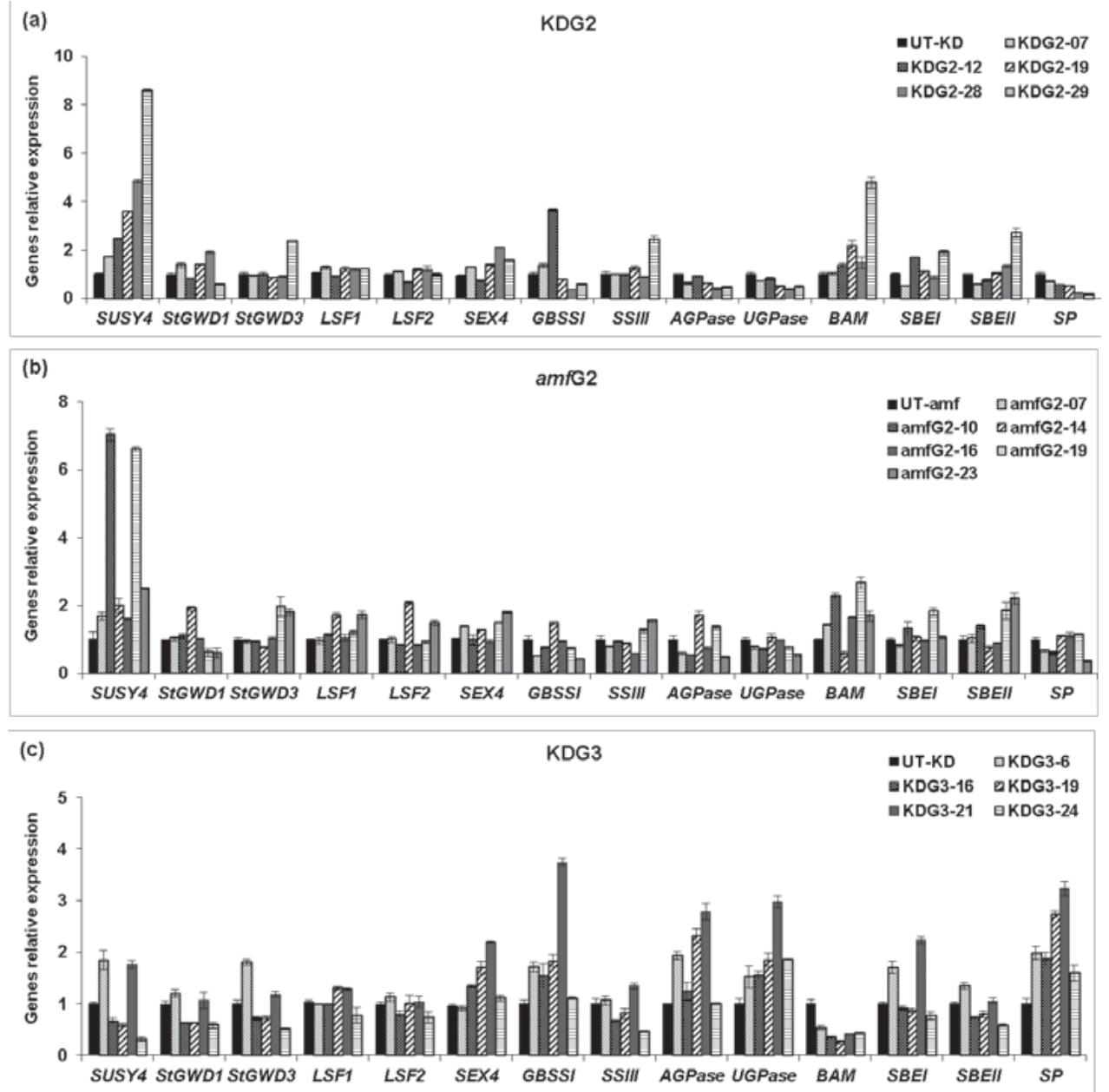

(d)

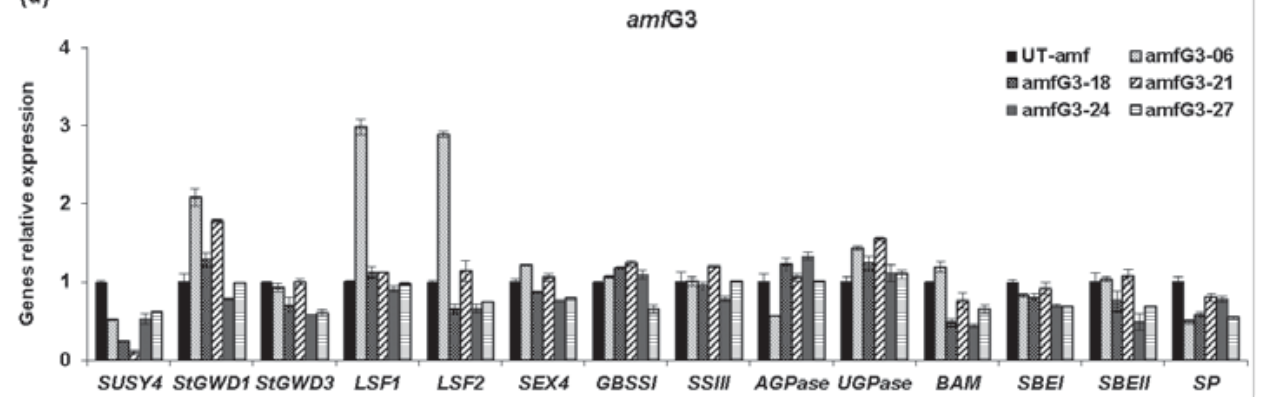

FIGURE 8. The expression of genes encoding key enzymes involved in starch metabolism in the highexpressors of (a) KDG2, (b) amfG2, (c) KDG3 and (d) amfG3 transgenic series. Following genes were investigated: sucrose synthase 4 (SUSY4), glucan, water-dikinase 1 and 3 (StGWD1 and StGWD3), starch phosphatase (LSF1, LSF2 and SEX4), granule-bound starch synthase I (GBSSI), starch synthase (SSIII), ADP-glucose pyrophosphorylase (AGPase), UDP-glucose pyrophosphorylase (UGPase), $\beta$-amylase (BAM), starch branching genes (SBEI and SBEII), starch phosphorylase (SP). The expression of target genes was expressed relative to the expression of elongation factor EF1a. Data (means \pm SD of three technical replicates) are expressed as the mean fold change relative to the control. 


\section{Discussion}

A new phylogenetic tree has been constructed including 28 new GWDs, comprising all identified homologues of GWDs up to now. GWD2 and GWD3 were found in a wide range of organisms, while GWD2 was identified in very few higher plants, corroborating previous reports (Baunsgaard et al., 2005; Glaring et al., 2007). Unlike previously described (Glaring et al., 2007), GWD2 is not exclusive to dicots but also present in monocots, such as Brachypodium distachyon and Hordeum vulgare (Blennow et al., 2013; Radchuk et al., 2009; Tanackovic et al., 2014b). This result confirmed the assumption that the duplication of GWD occurred before divergence of monocots and dicots. Moreover, the study on AtGWD2 null mutants revealed that plant growth or the level of starch and sugar was comparable to the wild type (Glaring et al., 2007), indicating that this gene has little or no effects on plant survival and development. It has been shown that the biological (ir) relevance of a gene is correlated with their loss during evolution (Krylov et al., 2003). Together these considerations make it tempting to speculate that GWD2 might have been lost in most of higher plants during the course of evolution, and this might explain the restricted occurrence of GWD2.

AtGWD2 was demonstrate to exhibit 50\% homology to GWD1 and phosphorylate C-6 position on elongated amylopectin chains in vitro as has been observed for both AtGWD1 and StGWD1 (Glaring et al., 2007). Unlike GWD1, it locates in companion cells of the phloem rather than chloroplast due to the lack of the transit peptide. In our study, AtGWD2 was targeted into amyloplast using GBSSI transit peptide and can thereby be expected to serve as an equivalent enzyme of GWD1. However, expression of this gene into potato tuber did not lead to an increase in phosphate content of tuber starch. In fact, the function of AtGWD2 has not yet been elucidated in vivo. In Arabidopsis thaliana, the target for the action of AtGWD2 was speculated to be the starch granules in the plastids of phloem sieve elements. These sieve element starches have been shown to have a different structure from ordinary starches (Palevitz and Newcomb, 1970), and the environment in the plastids of arabidopsis phloem sieve elements might be distinct from that in the potato amyloplast (e.g. pH, salt concentrations, etc.). Hence, one possible explanation for the no further phosphorylation of starch could be that the substrate and/or environment was not amenable for AtGWD2 activity in potato amyloplast.

On the other hand, assuming that AtGWD2 is capable of phosphorylating potato starch during starch synthesis, the question remains as to whether it is possible to increase the phosphate content of potato starch without changes in starch molecular structure. It has been shown that potato tuber starches are already highly phosphorylated (up to 33 $\mathrm{nmol} / \mathrm{mg}$ ) compared with transitory and cereal endosperm starches (less than $1 \mathrm{nmol} /$ mg) (Baunsgaard et al., 2005; Blennow, 2015). To date, potato starches with increased phosphate content have been attained as a side effect due to altered amylopectin 
structure or decreased amylose content. It is likely that there are limiting factors for the incorporation of phosphate groups in starch rather than the phosphorylating enzyme itself. Therefore, the measured phosphate content might be the maximum amount achievable in potato starch, which could possibly explain why transformants with AtGWD2 expression do not lead to increase in starch phosphate content. We suggest that a further increase in phosphate content is only possible when more places are available to deposit phosphate groups in the starch granules resulting from a change in the starch molecular structure. A similar phenomenon has been observed in earlier studies, demonstrating that introduction of additional copies of the granule-bound starch synthase (GBSS) gene into potato plants did not increase amylose content above the wild-type level (Flipse et al., 1994; Flipse et al., 1996a). The authors reasoned that the restricted amount of amylose content was due to the limited space existed to deposit amylose molecules in starch granules.

Similar to the results observed in the AtGWD2 transformants, AtGWD3 transformants did not show significant change in the phosphate content of tuber starches. The explanations provided above may also apply in this case. It is worth noting that higher starch phosphate content has been obtained in Arabidopsis thaliana by overexpression AtGWD3 (Frohberg et al., 2013), however, transitory starches, the substrate for AtGWD3, are considerably different from storage starches, such as amylose/amylopectin ratio, size and chemical structure of both these components.

The granule morphology was altered in modified starches compared to the control (Fig. 6). This is likely due to the presence of the newly introduced proteins in granules during starch synthesis, interfering with lateral interactions of amylopectin side chains and thereby interrupting the optimal crystalline packing of starch granules. This phenomenon has been observed in previous studies by introducing granule-bound proteins into amyloplasts (chapter 3, 4 and 5) (Firouzabadi et al., 2007; Huang et al., 2013; Huang et al., 2014). The larger granules observed in KD modified starches may be partly attributed to the irregular geometrical shape of the granules.

The expression of key genes involved in the starch metabolism pathway generally did not show significant alterations in any of the transgenic series, except for SUSY4. In AtGWD2 transformants in both backgrounds, SUSY4 expression had a substantial increase (Fig. 8a and b); however, we cannot find a conclusive explanation so far. An early study has shown that SUSY4 is a major determinant of tuber sink strength and the suppression of this gene leads to an inhibition of starch accumulation in potato tubers (Zrenner et al., 1995). In Chapter 4, the inhibition of GWD1 expression led to the downregulation of a series of key genes including SUSY4, thus we hypothesized that starch phosphorylation affects starch metabolism partly by regulating the flux into the amyloplast. Further experiments are needed to examine if the expression of AtGWD2 directly or indirectly triggers such regulation in potato tuber. 


\section{Supplementary data}

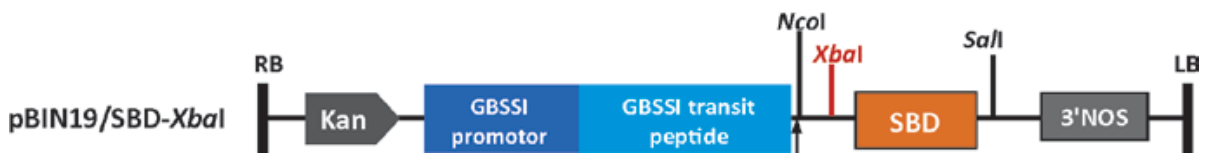

FIGURE S1. Schematic representation of pBIN19/SBD-Xbal vector. This construct was modified based on pBIN19/SBD (Ji et al., 2003) by adding an Xbal restriction site. RB and LB represent right and left borders, respectively. SBD, Kan and 3'NOS stand for starch binding domain of cyclodextrin glycosyltransferase from Bacillus circulans, kanamycin resistant gene and NOS terminator, respectively. Ncol, Sall and Xbal are restriction enzymes. 


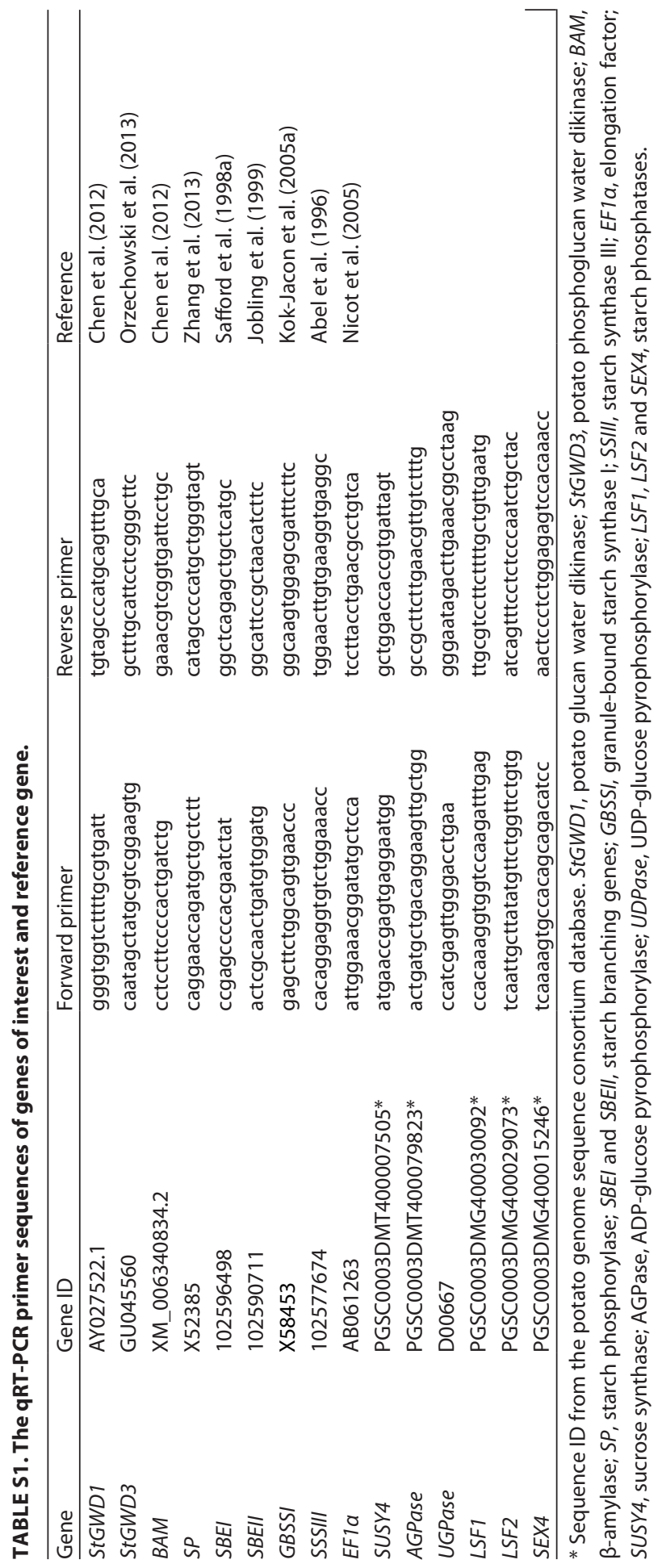




\title{
Chapter 6
}

\section{Expression of an (engineered) 4,6-a-glucanotransferase in potato results in changes in starch characteristics}

\author{
Xuan $\mathrm{Xu}^{1,2}$, \\ Annemarie Dechesne ${ }^{1}$, \\ Richard G.F Visser ${ }^{1}$, \\ Luisa M. Trindade ${ }^{1^{*}}$
}

${ }^{1}$ Wageningen UR Plant Breeding, Wageningen University and Research, P.O. Box 386. 6700AJ Wageningen, The Netherlands.

${ }^{2}$ National Centre for Vegetable Improvement (Central China), Key Laboratory of Horticultural Plant Biology, Ministry of Education, Huazhong Agricultural University, Wuhan, 430070, China. 


\begin{abstract}
Starch structure strongly influences starch physicochemical properties, determining the end uses of starch in various applications. To produce starches with novel structure and exploit the mechanism of starch granule formation, an (engineered) 4, 6-a-glucanotransferase (GTFB) from Lactobacillus reuteri 121 was introduced into two potato genetic backgrounds: amylose-containing line Kardal and amylose-free mutant amf. The resulting starches showed severe changes in granule morphology regardless of genetic backgrounds. Modified starches from amf background exhibited a significant increase in granule size and starch phosphate content relative to the control, while starches from Kardal background displayed a higher digestibility, but did not show changes in granule size and phosphate content. Transcriptome analysis revealed the existence of a mechanism to restore the regular packing of double helices in starch granules, which possibly resulted in the removal of novel glucose chains potentially introduced by the (engineered) GTFB. This amendment mechanics would also explain the difficulties to detect alterations in starch fine structure in the transgenic lines.
\end{abstract}




\section{Introduction}

Starch, the most important source of calories in human diet, is the major storage carbohydrate in various photosynthetic tissues and storage organs. Starches from different botanic origins vary in granule shape and size, starch composition and structure as well as their properties. Notwithstanding these differences, the composition of native starches is universally composed of amylopectin (70-80\%) and amylose (20-30\%) with some minor components such as lipids and proteins (reviewed in (Xu et al., 2014)). Amylose is mostly a linear molecule formed by a-1,4-linked glucose residues and less than $1 \%$ a-1,6 branching points, whereas amylopectin is a highly branched molecule with 4-5\% a -1,6 linkages. These two components are packed in ordered arrays within the granule, giving rise to alternating semicrystalline and amorphous growth rings (Jenkins et al., 1993a). However, mechanisms underlying starch granule formation remain unclear, especially in storage starches.

Over the past decade, in planta production of starches with novel properties using genetic modification has attracted particular attention, as it potentially generates environmental and economic benefits and broadens starch end-uses in industrial applications (Kaur et al., 2012; Moire et al., 2003; Snell et al., 2015). Many studies focused on the alteration in starch structure due to its great effect on starch properties, such as gelatinization properties, swelling power, pasting properties (Fredriksson et al., 1998; Jane et al., 1999; Tan and Halley, 2014). Efforts have been made to introduce changes in amylopectin fine structure by modulating endogenous gene expression in different species (Blauth et al., 2002; Katayama et al., 2002; Safford et al., 1998b; Satoh et al., 2003; Umemoto et al., 2002). For instance, simultaneous downregulation of two starch synthases (SSII and SSIII) in potato resulted in enrichment in shorter chains and a depletion in longer chains of amylopectin, which ultimately affects starch gelatinization temperature and viscosity (Edwards et al., 1999). Moreover, downregulation of three starch synthases (GBSSI, SSII and SSIII) generated an amylose-free starch with short-chain amylopectin, which showed high freeze-thaw stability (Jobling et al., 2002). On the other hand, expression of heterologous genes in potato has proven to have great potential to modify starches in planta (Xu et al., 2014). These genes may have properties that are slightly different from their plant counterparts and thus create different or novel phenotypes. An example of this is the study carried out by Kortstee et al. (1996) in which Escherichia coli glycogen branching enzyme has been introduced in amylose-free potato mutant, resulting in $25 \%$ higher branching degree of amylopectin.

A 4,6-a-glucanotransferase from Lactobacillus reuteri 121 (GTFB) is a novel enzyme that can convert starch or starch hydrolysates into isomalto/maltopolysaccharides (IMMPs) (Dobruchowska et al., 2012). This enzyme can transfer the non-reducing glucose moiety of an $a-1,4$ glucan chain to the non-reducing end of another a -glucan through $a-1,6$ 
linkages, generating a linear chain with a-1,6 linkages (Bai et al., 2015; Leemhuis et al., 2014). This specific activity makes GTFB an interesting target enzyme for producing novel starches in planta.

In this study, GTFB either alone or fused to a starch-binding domain (SBD) has been introduced into amylose-containing (Kardal) and amylose-free mutant (amf) potato genetic backgrounds. The effects of the (engineered) GTFB on starch characteristics and starch biosynthetic pathway are presented and discussed.

\section{Materials and methods}

\section{Construction of plasmids}

GTFB was amplified from genomic DNA of Lactobacillus reuteri 121 with the forward primer $\mathrm{F}\left(5^{\prime}-\mathrm{ATGGAACTCAAAAAACATTTAAGC}-3^{\prime}\right)$ paired with the reverse primer $\mathrm{R}\left(5^{\prime}-\right.$ TTAGTTGTTAAAGTTIAATGAAATTG-3'). Two constructs were made in this study (Fig. 1). One construct, pBIN19/GB, was used for the expression of GTFB; another construct, pBIN19/ $S G B$, was used for the expression of fusion protein, in which GTFB was fused in-frame to the starch-binding domain (SBD) of Bacillus circulans cyclodextrin glycosyltransferase at the carboxyl terminus.

(a) $\mathrm{pBIN19/GB}$

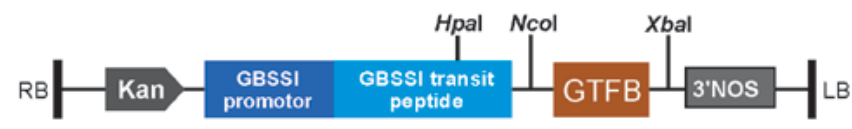

(b) pBIN19/SGB

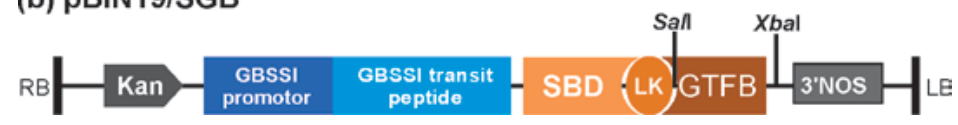

FIGURE 1. Schematic depiction of two different binary vector constructs (a) pBIN19/GB and (b) pBIN19/ SGB. Genes were cloned in frame with GBSSI transit peptide to allow amyloplast targeting and were driven by the potato GBSSI promoter for tuber expression. RB and LB represent right and left borders, respectively. SBD, LK, Kan and 3'NOS stand for starch binding domain of cyclodextrin glycosyltransferase from Bacillus circulans, linker, kanamycin resistant gene and NOS terminator, respectively. Sall, Hpal, Ncol and Xbal are restriction enzymes.

The pBIN19/GB plasmid was constructed by using the amplified fragment to substitute the SBD2 fragment in the pBIN19/SBD2-Xbal vector (Supplementary Fig. S1). The fragment consisted of two parts: a part of transit peptide and full-length GTFB gene. To generate this fragment, two starting fragments with overlapping ends were amplified with the forward primer TP-F (5'-ATAGTTAACAAGCTTGATGGGCTCCAATC-3') paired with the reverse 
primer TP-R (5'-CTTAAAATGTIITTGAGTTCCATGGTACAAACAATGGTAGCTGAG-3'), and forward primer GB-F (5'-AGCTACCATTGTTTGTACCATGGAACTCAAAAAACATTTAAGC $\left.-3^{\prime}\right)$ paired with the reverse primer GB-R (5'-CTTCTAGATTAGTTGTTAAAGTTTAATGAAATTGCAG-3'). These PCR products were mixed in a final PCR reaction to generate the full-length fragment with primers TP-F and GB-R. The resulting fragment was digested with Hpal and Xbal and ligated sequentially into pBIN19/SBD2-Xbal to generate pBIN19/ GB (Fig. 1a).

To generate the pBIN19/SGB plasmid, a GTFB-encoding fragment was obtained by PCR amplification with the primers 5'-GCGTCGACATGGAACTCAAAAAA-3' and 5'-CTAGTCTAGATTAGTTGTTAAAGTITAATGAAATT-3', which contained Sall and Xbal sites at their $5^{\prime}$ ends, respectively. This amplified GTFB fragment was digested with Sall and Xbal and ligated into pBIN19/SBD2-Xbal vector, generating the pBIN19/SGB construct (Fig. 1b).

\section{Transformation and regeneration}

Four-week-old shoots from amylose-containing potato (cv. Kardal, tetraploid) and amylosefree mutant ( $a m f$, diploid) were used for Agrobacterium-mediated transformation (Visser et al., 1991). In this study, transgenic plants were labelled KDGBxx, KDSGBxx, amfGBxx and $a m f S G B x x$ in which KD and amf represent Kardal and amf background, GB and SGB stand for GTFB and SBD-GTFB, respectively, xx represents the number of the transgenic line. Transgenic shoots were selected on a rooting medium containing kanamycin at concentrations of $100 \mathrm{mg} / \mathrm{L}$, followed by PCR-based selection using primers specific for the kanamycin resistance gene (NPTII) and the target gene (GTFB). Ultimately, 32, 27, 28 and 19 independent plantlets from KDGB, KDSGB, amfGB and amfSGB series, respectively, were generated. Five clones of each line were multiplied by culturing nodal explants according to the procedure described in (Visser et al., 1991). These transformants as well as control plants (untransformed plants and transformed plants with empty vector) were further grown in the greenhouse under standard greenhouse conditions (16 h light at $20^{\circ} \mathrm{C}$ and $8 \mathrm{~h}$ dark at $18^{\circ} \mathrm{C}$ ) to generate mature tubers. No differences were detected between untransformed lines and transformed plantlets with empty vector, and they are further referred to as controls (UT-KD and UT-amf).

\section{Starch isolation}

To minimize individual variation, tubers from five plants from the same clone were pooled together, and starch isolation was performed according to the procedure described in (Huang et al., 2013). 


\section{Western dot blot analysis}

Fifty $\mathrm{mg}$ of starch were heated at $100^{\circ} \mathrm{C}$ for 5 min with $400 \mu \mathrm{l}$ of a $2 \times$ SDS sample buffer containing $5 \% \beta$-mercaptoethanol (Laemmli, 1970). After cooling to room temperature, the supernatant was transferred to a 96-well format dot-blot manifold (Schleicher \& Schuell, Keene, NH). The dot-blot manifold was connected to a water pump, and a vacuum was applied for $5 \mathrm{~min}$ until all samples were impacted on the nitrocellulose membrane (Bio-rad).

The dot blots were blocked overnight at $4^{\circ} \mathrm{C}$ in Tris-buffered saline (TBS is $20 \mathrm{mM}$ Tris, $500 \mathrm{mM} \mathrm{Nacl}$ pH 7.5) with $0.1 \%$ Tween-20 (TBST) containing 5\% (w/v) dry powdered nonfat milk. Membranes were then incubated for $2 \mathrm{~h}$ at room temperature with a 1:1000 dilution of the anti-SBD antibody (Ji et al., 2003) in 3\% non-fat milk in TBST, followed by 5 rinses in TBST. The membrane was then incubated with a 1:5000 dilution of horse radishperoxidase-conjugated anti-(rabbit lgG) (Cat \# A0545, Sigma) in TBST buffer with 3\% (w/v) of non-fat milk for $1 \mathrm{~h}$ at room temperature. After rinsing 5 times in TBST, the protein was detected with a West Femto supersignal (Cat \# 34094, Thermo Scientific).

\section{Analysis of starch characteristics}

All analyses conducted in this study have been performed in duplicate unless indicated otherwise.

Particle size distribution and gelatinization properties of starches were analysed with Coulter Multisizer II (Beckman-Coulter, UK) and Differential Scanning Calorimetry (DSC), respectively. Samples were analysed according to the procedure used by Ji et al. (2004).

Amylopectin chain length distribution was determined by using high-performance anion-exchange chromatography with pulsed amperometric detection (HPAEC-PAC). Degree of polymerisation (DP) 6 - 35 were separated according to the method described in Huang et al. (2013). To detect linear a-1, 6 chains in starch structure, $5 \mathrm{mg}$ of starch was suspended in $250 \mu \mathrm{l}$ of DMSO and gelatinized for $15 \mathrm{~min}$ at $99^{\circ} \mathrm{C}$. After cooling down, 745 $\mu \mathrm{l}$ of $\mathrm{NaAc}$ buffer (100 mM, pH 5.6) was added, followed by incubation with a sufficient amount of dextranase (Sigma) overnight at $50^{\circ} \mathrm{C}$. The resulting products were analysed using HPAEC-PAC.

The apparent amylose content was performed according to the procedure described in Hovenkamp-Hermelink et al. (1988).

Starch content was determined as described by Kok-Jacon et al. (2005a).

Total phosphate content in the starch was determined essentially according to the method of Morrison (1964) with some modifications. About $20 \mathrm{mg}$ of dry starch were suspended in $250 \mu \mathrm{l}$ of $70 \%(\mathrm{w} / \mathrm{w}) \mathrm{HClO}_{4}$ and completely charred at $250{ }^{\circ} \mathrm{C}$ for $25 \mathrm{~min}$. 
The solution was clarified by adding $50 \mu \mathrm{l} 30 \%(\mathrm{w} / \mathrm{v}) \mathrm{H}_{2} \mathrm{O}_{2}$ and gently boiled for 2 min. Once the solution had cooled, water was added to a final volume of $2 \mathrm{ml}$ and $100 \mu \mathrm{l}$ of the sample was transferred into a 96-well microtiter plate, followed by adding $200 \mu \mathrm{l}$ of colour reagent $\left[0.75 \%(\mathrm{w} / \mathrm{v})\left(\mathrm{NH}_{4}\right)_{6} \mathrm{Mo}_{7} \mathrm{O}_{24} \cdot 4 \mathrm{H}_{2} \mathrm{O}, 3 \%(\mathrm{w} / \mathrm{v}) \mathrm{FeSO}_{4} \cdot 7 \mathrm{H}_{2} \mathrm{O}\right.$ and $0.75 \%(\mathrm{w} / \mathrm{v})$ SDS dissolved in $0.375 \mathrm{M} \mathrm{H}_{2} \mathrm{SO}_{4}$ ]. Absorbance at $750 \mathrm{~nm}$ was then measured in a Model 680 XR Microplate Reader (Bio-Rad, US), and compared to the absorption of a calibration curve to calculate the concentration in $\mathrm{nmol} \mathrm{PO}_{4} / \mathrm{mg}$ starch.

Starch digestibility was determined essentially according to the method of Warren et al. (2015) with some modifications. Fifty milligrams of starch was suspended in $4.95 \mathrm{~mL}$ of acetate buffer $\left(0.2 \mathrm{M}\right.$, pH 6, containing $200 \mathrm{mM} \mathrm{CaCl}_{2}$ and $0.5 \mathrm{mM} \mathrm{MgCl}$ ), followed by incubation with $50 \mu \mathrm{l}$ enzyme solution containing $10 \mathrm{U}$ a-amylase (porcine pancreas, Sigma) and 5.6 $\mathrm{U}$ amyloglucosidase (Aspergillus niger, Megazyme) for $2 \mathrm{~h}$ at $37^{\circ} \mathrm{C}$. Aliquots $(200 \mu \mathrm{L})$ were centrifuged and subjected to HPAEC-PAC. The amount of glucose was calculated and presented as microgram per $1 \mathrm{mg}$ dry starch.

\section{RNA isolation and quantitative RT-PCR (qRT-PCR) analysis}

Mature tubers were sampled from five plants from the same clone. Samples were frozen in liquid $\mathrm{N}_{2}$ and stored in $-80^{\circ} \mathrm{C}$ freezer for RNA isolation. Total RNA was extracted from potato tuber samples according to Kuipers et al. (1994). cDNA was synthesized from $1 \mu \mathrm{g}$ of total RNA, previously treated with RNase-free DNase I (Invitrogen), by using the iScript ${ }^{\mathrm{TM}}$ CDNA Synthesis Kit (Bio-Rad). Quantitative real time PCR (qRT-PCR) was performed using CFX96 Real-Time PCR machine (BioRad). The total volume of each reaction was $10 \mu$, containing $50 \mathrm{ng}$ cDNA, $3 \mu \mathrm{M}$ of each gene-specific primer, and $5 \mu$ I SYBR Green Supermix Reagent (BioRad). All reactions were carried out in triplicate using the following thermal cycling conditions: $3 \mathrm{~min}$ of denaturation at $95^{\circ} \mathrm{C}$, followed by 45 cycles $\left(15 \mathrm{~s}\right.$ at $95^{\circ} \mathrm{C}, 60 \mathrm{~s}$ at $60^{\circ} \mathrm{C}$ ). The elongation factor 1 - $a$ gene $(E F 1 a$ ) was used as the reference to normalize gene expression across the samples because it was reported to be relatively stable in expression (Nicot et al., 2005). Target genes were expressed relative to ef1a using the comparative $\mathrm{Ct}$ method (Livak and Schmittgen, 2001). Gene-specific primers were designed with Primer3-Plus software (Untergasser et al., 2007b) and are listed in Supplementary Table S1.

Expression level of (engineered) GTFB from all transformants was determined. The relative expression level of target genes was multiplied by a factor of $10^{4}$ (for KDSGB and amfSGB series) or $10^{5}$ (for KDGB and amfGB series) and then converted to log 10. Ultimately, the resulted value $(\mathrm{v})$ was used to divide transformants to different categories: undetectable $(\mathrm{N}, \mathrm{v}=0)$, low $(\mathrm{L}, 0<\mathrm{v}<2)$, medium $(\mathrm{M}, 2 \leq \mathrm{v}<2.5)$ and high $(\mathrm{H}, \mathrm{v} \geq 2.5)$ expressors.

Three or four $\mathrm{H}$-expressors from each series were used for investigating expression of key genes involved in starch metabolism. These genes were glucan water-dikinase (GWD1), 
starch phosphorylase (SP), isoamylase genes (ISA1, ISA2 and ISA3), starch branching genes (SBEI and SBEII), starch synthase (SSIII), $\beta$-amylase (BAM) and ADP-glucose pyrophosphorylase (AGPase). The gene specific primers used in this study are listed in Supplementary Table S1.

\section{Statistical analysis}

Significant differences between modified starches and control samples in the present study were assessed by one-way analysis of variance (ANOVA). The least significant difference values were calculated at $1 \%$ or $5 \%$ probability.

\section{Results}

\section{(Engineered) GTFB is expressed in transformants}

Two constructs were introduced into two potato genetic backgrounds, KD and amf, respectively. None of transgenic lines showed significant phenotypic differences in the above ground nor underground tissues relative to control plants.

For KDSGB series, qRT-PCR and Western dot blot analysis were performed on all transformants to examine SBD-GTFB expression level and the protein abundance in starch granules, respectively. The results showed that the dot intensity, which corresponds to the protein amount, was well correlated with the gene expression level (Fig. 2a). For this reason, and also because no antibody is available for the detection of GTFB, transformants from other series were only investigated using qRT-PCR for gene detection and quantification (Fig. 2b).

Based on the transcript level, transformants from each series were grouped into four classes respectively: undetectable $(\mathrm{N})$, low $(\mathrm{L})$, medium $(\mathrm{M})$ and high $(\mathrm{H})$ expressors. Several $\mathrm{H}$-expressors from each transgenic series were selected for further detailed description. From the KDGB series, KDGB20, KDGB26 and KDGB33 were selected, and KDSGB3, KDSGB7, KDSGB16 and KDSGB24 were selected from the KDSGB series. In the amf background, amfGB22, amfGB24, amfGB25, amfSGB1, amfSGB4, amfSGB12 and amfSGB18 were selected for further characterization. As expected, no (engineered) GTFB expression was detected in the UT-KD and UT-amf lines (data not shown). 
(a)
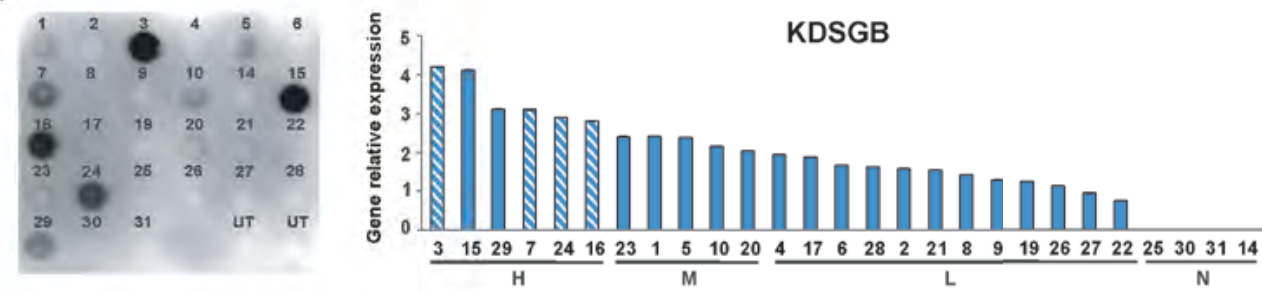

(b)
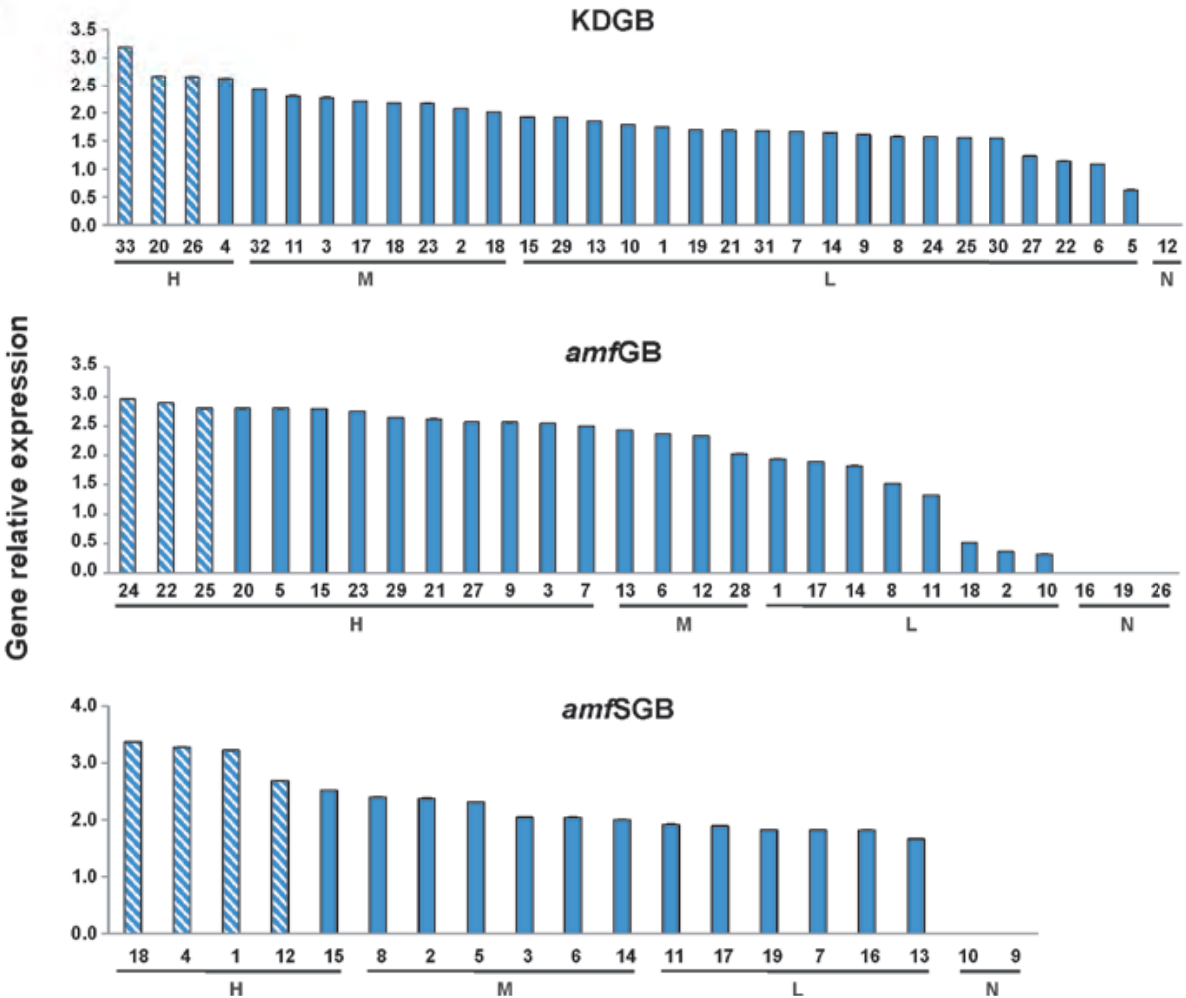

FIGURE 2. Different levels of gene expression in transgenic lines. (a) Protein accumulation level and gene expression level in KDSGB transformants. The protein accumulation and gene expression level were determined using Western dot blot with an anti-SBD antibody and qRT-PCR analysis, respectively. The number above each dot stands for the different line, while UT represents the control. The intensity of the dots shows the various protein levels. The qRT-PCR analysis was performed in triplicate for each line and the relative expression level of target genes was transformed as described in the Materials and Methods. The resulting value $(v)$ was used to divide transformants to different categories: undetectable $(N, v=0)$, low $(L, 0<v<2)$, medium $(M, 2 \leq v<2.5)$ and high $(H, v \geq 2.5)$ expressors. (b) Distribution of the individual transformants over the classes of gene expression level in KDGB, amfGB and amfSGB transgenic series. SGB and GB represent SBD-GTFB fusion protein and GTFB alone, respectively. KD stands for the Kardal background, while amf stands for the amf background. The striped columns indicate the lines selected for further characterization. 


\section{Starch granule morphology is altered in transformants}

Light microscopy (LM) and scanning electron microscopy (SEM) were used to investigate the morphology of starch granules. As shown in Fig. 3, starch granule morphology was affected by the expression of the (engineered) GTFB in both genetic backgrounds. In the KD background, starch granules with bumpy and irregular shape were observed in starches from KDGB (Fig. 3E, F, G and H) and KDSGB transformants (Fig. 3I, J, K and L), while UT-KD granules showed a regular rounded form and smooth surface (Fig. 3A, C and D). The growth rings in starch granules of the transformants exhibited much less concentric and regular patterns than those of the UT-KD (Fig. 3B, F and J); in particular, multiple initiation sites were observed in some starch granules (Fig. 3F). In some of the KDSGB starches craters were observed in the surface of the starch granules (Fig. 3L).

In the amf background, amfGB starches exhibited irregular round-shaped granules (Fig. $3 \mathrm{Q}$ and S), and some of these granules showed blister-like spots protruding from the surface (Fig. 3T). The shape of amfSGB starches granule was comparable to the control (Fig. $3 \mathrm{U}$ and $\mathrm{W}$ ), however, granules were pitted with individual pores with varying size and depth (Fig. 3X) rather than the smooth surface observed in the control (Fig. 3P). These pores were only present on the granule surface and did not penetrate through the whole granule. Moreover, growth rings in starch granules from both amfGB and amfSGB transformants were also not distributed as evenly as in the control UT-amf (Fig. 3R and V), as observed in the KD background.

To examine whether the expression level of the transgene affects the percentage of altered granules, we counted a population of 100 granules in triplicate for starches from transgenic lines that belonged to different expression classes. As shown in Fig. 3b, the highest numbers of altered starch granules were observed in the H-expressors from each series. In particular, $\sim 80 \%$ of starch granules showed morphological alteration in $\mathrm{H}$-expressors (KDSGB3 and amfSGB18) from both KDSGB and amfSGB series. For the $\mathrm{N}$-expressors, the frequency of altered starch granules was less than $8 \%$ in all series. Overall, a positive correlation was found between transgene expression and the percentage of altered granules regardless of the construct and background used. 
(a)
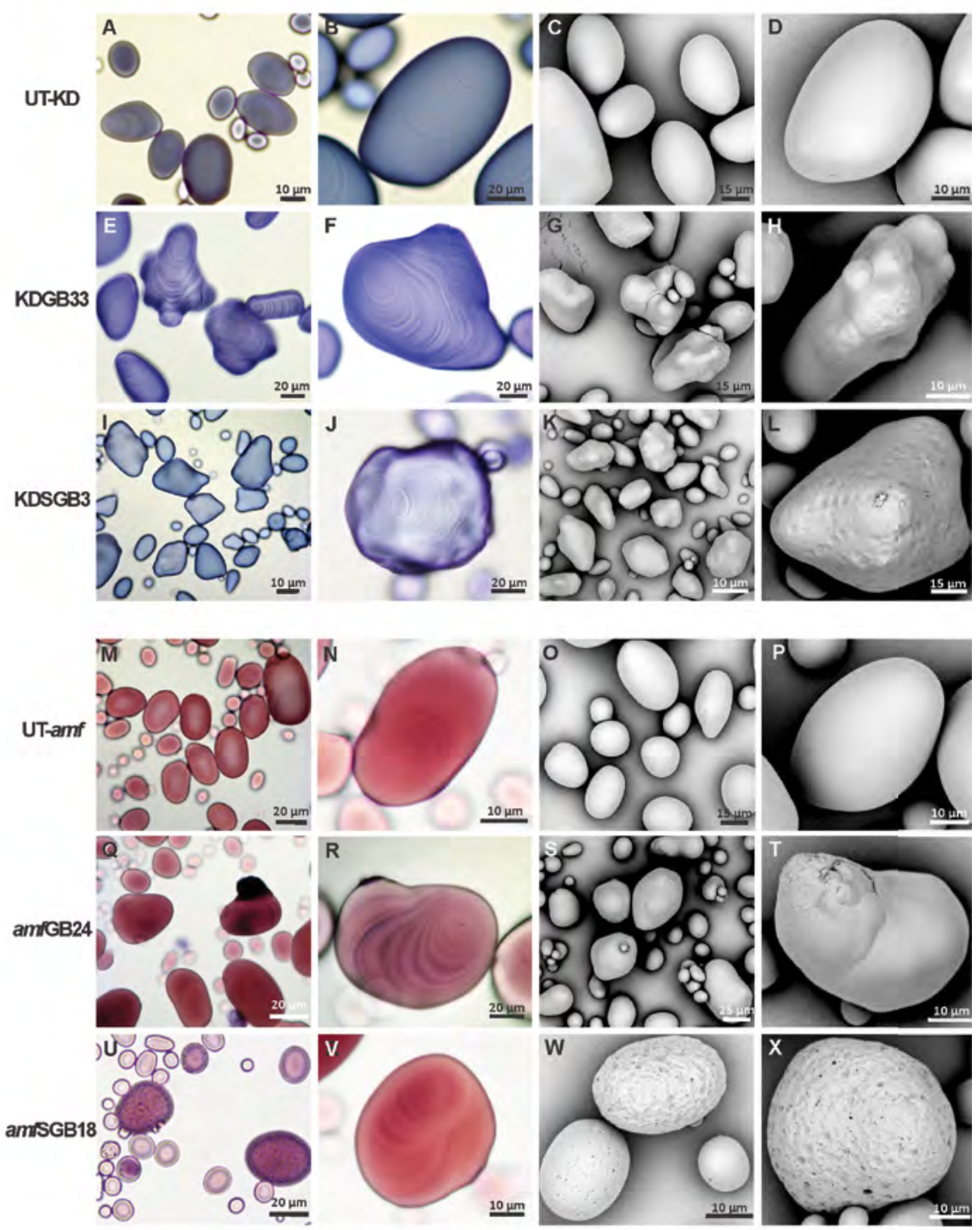

FIGURE 3. Granule morphology of starches from transformants and controls. (a) Light microscopy (LM) and scanning electron microscopy (SEM) of starch granule morphology from UT-KD (A, B, C and D), KDGB33 (E, F, G and H), KDSGB3 (I, J, K and L) UT-amf (M, N, O and P), amfGB24 (Q, R, S and T) and amfSGB18 (U, V, W and X). Starch granules were stained with a $20 \times$ diluted Lugol solution for light microscopy. 
(b)

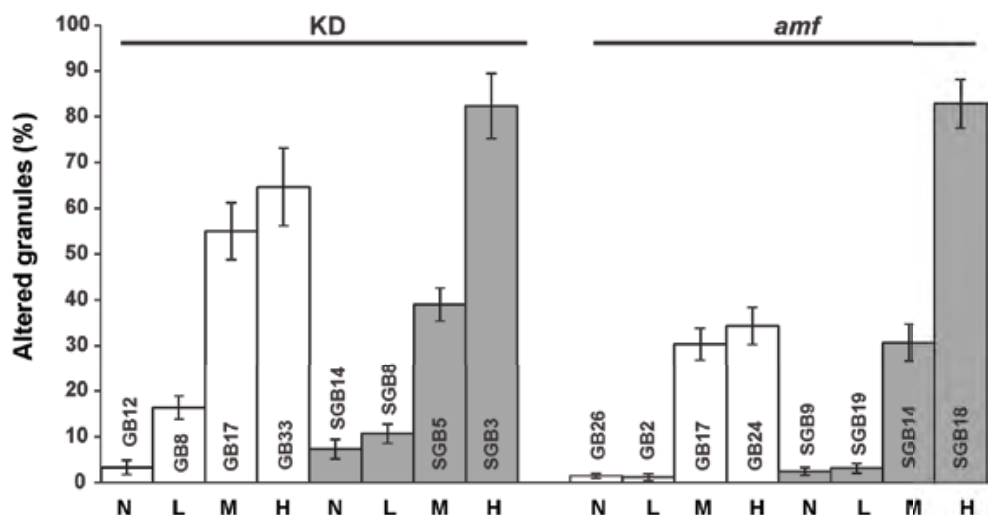

FIGURE 3 Continued. Granule morphology of starches from transformants and controls. (b) Percentage of granules with altered morphology for the various classes of gene expression level: not detected $(N)$, low $(L)$, medium (M) and high (H). GB and SGB are abbreviations for GTFB and SBD-GTFB, respectively. A population of 100 starch granules was counted in triplicate. The values are expressed as the mean \pm SD from three independent counts.

\section{Modified starches from the amf background have bigger granules and higher phosphate content}

Granule size distribution was analysed for all starches from transformants and control plants. The results showed that starches from amfSGB series had significantly larger granules than the control (Fig. 4a, ANOVA, $p<0.01$ ). As shown in Fig. 4b, starch granule size increased up to two fold in $\mathrm{H}$-expressors relative to the control. By contrast, no significant differences were found between starch granule size of transformants and that of the control in the KD background (Fig. 4a).

In the amf background, modified starches showed a significant increase in phosphate content (ANOVA, $p<0.01$ ), as the average amount in each series was $~ 19 \%$ higher than that of the control (Fig. 5a). As shown in Fig. 5b, the phosphate content of starches from $\mathrm{H}$-expressors was significantly increased (t-test, $p<0.01$ or 0.001 ) relative to that of the control line regardless of constructs. On the other hand, starches from KD background did not exhibit a significant difference in phosphate content between each transgenic series and the control (Fig. 5a). No consistent changes was found in starch phosphate content between $\mathrm{H}$-expressors and the control line (Fig. 5b). 
(a)

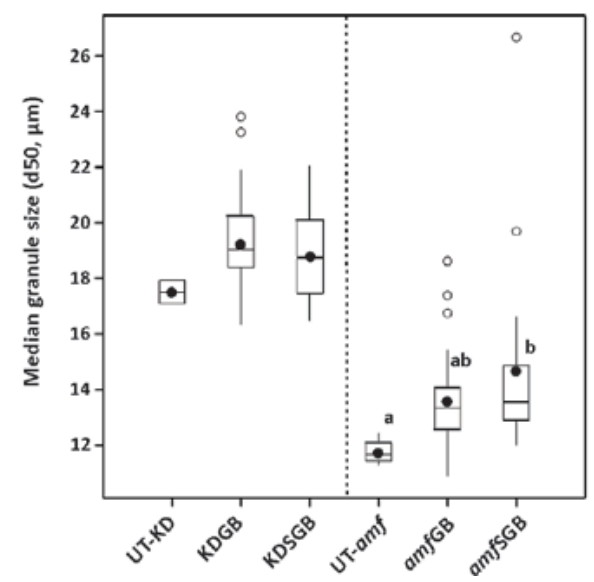

(b)

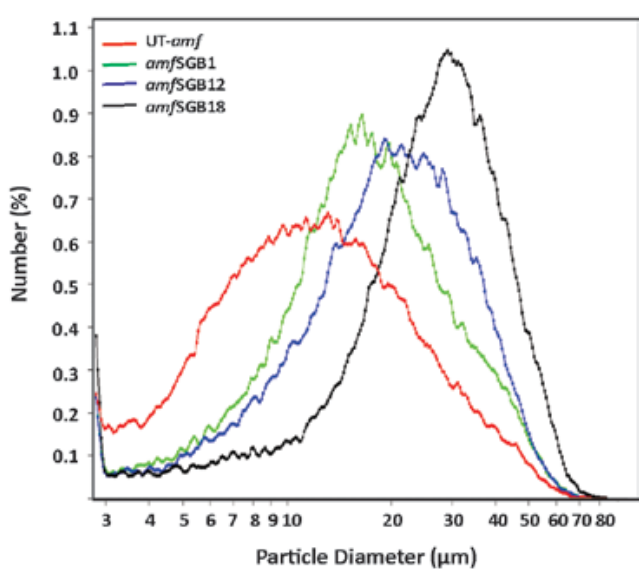

FIGURE 4. Starch granule size of starches from transgenic lines and control lines. (a) Boxplot presenting median granule size of modified starches and their respective controls. The measurements were performed on all transformants except $\mathrm{N}$-expressors (lines with undetectable expression of target gene), which are 31, 23, 25 and 17 lines for KDGB, KDSGB, amfGB and amfSGB series, respectively. Boxes in the plot include values in the 25\%-75\% interval. Internal lines, filled circles, unfilled circles and bars represent the median, the mean, outliers and extremes, respectively. Statistical significance was analysed using one-way ANOVA. Different letters indicate statistically significant differences between means at $p<0.01$. (b) Average particle size distribution of starches from amfSGB H-expressors (lines with high expression of target gene) and the control UT-amf. Each starch sample was analysed in duplicate. 
(a)

(b)
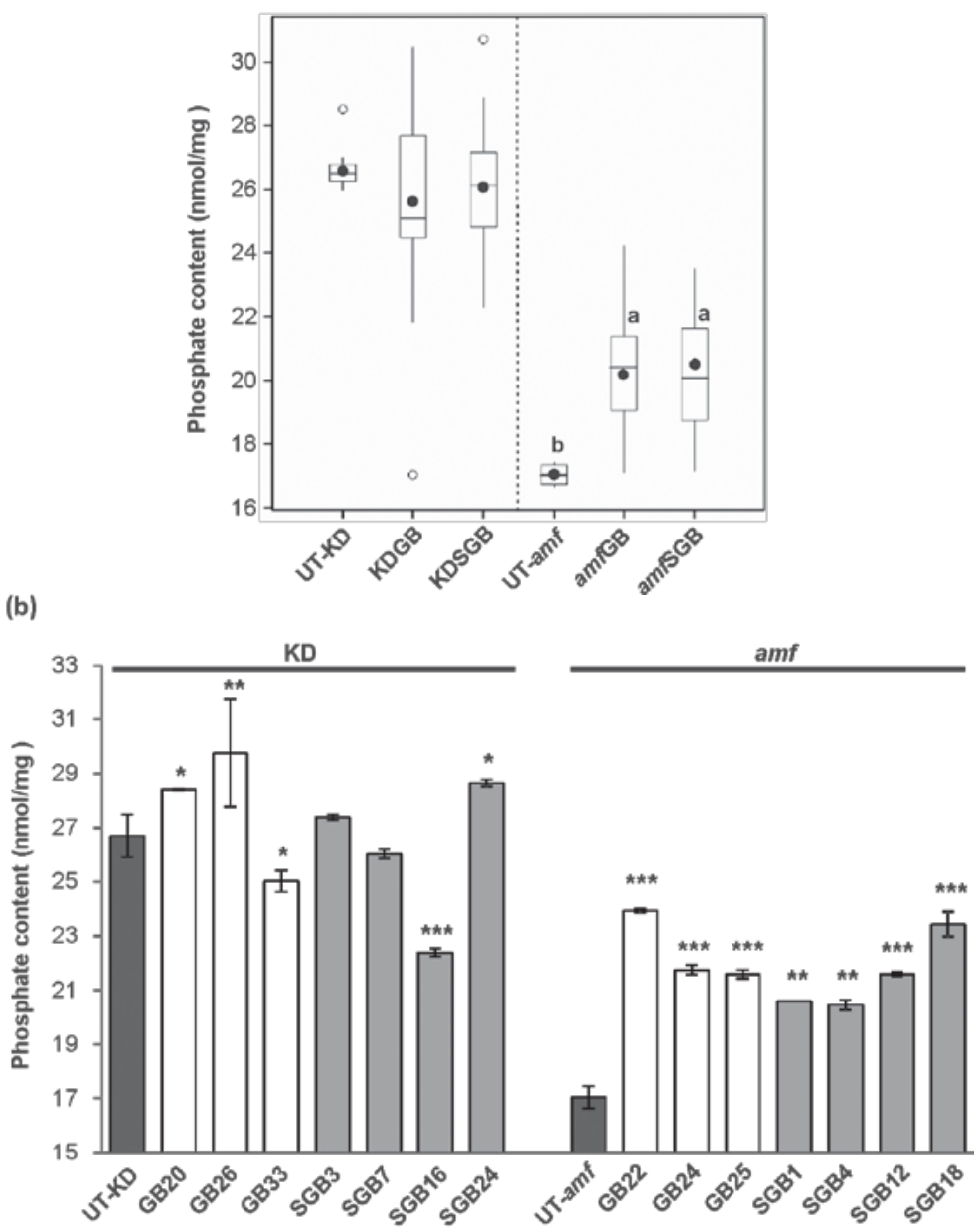

FIGURE 5. Phosphate content in starches from transgenic lines and control lines. (a) Boxplot presenting phosphate content in modified starches and their respective controls (UT-KD and UT-amf). The measurements were performed on starches from all transformants except $\mathrm{N}$-expressors (lines with undetectable expression of target gene), which are 31, 23, 25 and 17 lines for KDGB, KDSGB, amfGB and amfSGB series, respectively. Boxes in the plot include values in the $25 \%-75 \%$ interval. Internal lines, filled circles, unfilled circles and bars represent the median, the mean, outliers and extremes, respectively. Statistical significance was analysed using one-way ANOVA. Different letters indicate statistically significant differences between means at $p<0.01$. (b) Phosphate content in starches of $\mathrm{H}$-expressors (lines with high expression of target gene) from each transgenic series and control lines. Columns represent mean \pm SD from three independent measurements. Statistical significances between each starch sample and the control determined by using t-test $\left({ }^{*}, p<0.1 ;{ }^{* *}, p<0.01 ;{ }^{* * *}, p<0.001\right)$.

Amylose content and gelatinization properties were determined for all starches from transformants and their respective control. As presented in Table 1, generally, no significant differences were observed in these characteristics between modified starches and control starches (t-test). These results indicated that expression of the (engineered) GTFB does not affect these starch properties in both background. 
Chain length distributions of starches, after complete debranching with isoamylase, were analysed using HPAEC-PAC. The results did not show significant changes in both backgrounds (data not shown). To detect the presence of linear a-1, 6 chains in starch structure, starches were treated with dextranase and the amount of glucose and isomaltose were measured. No significant differences could be detected between products obtained from modified starches and control starches in both backgrounds (data not shown).

TABLE 1. Overview of characteristics determined for the starches from $\mathrm{H}$-expressors and controls in both backgrounds.

\begin{tabular}{lllllll}
\hline Clone & $\mathbf{P}(\mathbf{n m o l} / \mathbf{m g})$ & $\mathbf{A M}(\%)$ & $\mathbf{d 5 0}(\boldsymbol{\mu m})$ & $\mathbf{T}_{\mathbf{o}}\left({ }^{\circ} \mathbf{C}\right)$ & $\mathbf{T}_{\mathbf{c}}\left({ }^{\circ} \mathbf{C}\right)$ & $\boldsymbol{\Delta} \boldsymbol{H}(\mathbf{J} / \mathbf{g})$ \\
\hline UT-KD & $26.7 \pm 0.6$ & $18.3 \pm 0.3$ & $17.5 \pm 0.2$ & $66.9 \pm 0.2$ & $77.4 \pm 0.2$ & $16.5 \pm 0.1$ \\
KDGB20 & $28.4 \pm 0.0^{* *}$ & $19.4 \pm 0.2^{*}$ & $23.2 \pm 2.5$ & $65.9 \pm 0.3$ & $75.6 \pm 0.4^{*}$ & $16.6 \pm 0.3$ \\
KDGB26 & $29.8 \pm 2.0^{* * *}$ & $19.8 \pm 0.0^{*}$ & $21.8 \pm 2.1$ & $65.9 \pm 0.4$ & $74.3 \pm 0.5^{*}$ & $14.4 \pm 0.5^{*}$ \\
KDGB33 & $25.0 \pm 0.4^{*}$ & $19.5 \pm 0.2^{*}$ & $19.0 \pm 0.7$ & $66.1 \pm 0.3$ & $76.0 \pm 0.4$ & $15.3 \pm 0.3^{*}$ \\
KDSGB3 & $27.4 \pm 0.1$ & $19.1 \pm 0.1$ & $17.7 \pm 0.7$ & $66.1 \pm 0.1$ & $75.2 \pm 0.3^{*}$ & $16.7 \pm 0.7$ \\
KDSGB7 & $26.0 \pm 0.2$ & $20.0 \pm 0.3^{*}$ & $22.0 \pm 0.6^{* *}$ & $65.7 \pm 0.4$ & $74.4 \pm 0.2^{* *}$ & $16.0 \pm 0.2$ \\
KDSGB16 & $22.4 \pm 0.1^{* * *}$ & $18.5 \pm 0.1$ & $18.7 \pm 0.5$ & $66.1 \pm 0.1^{*}$ & $75.6 \pm 0.5$ & $14.6 \pm 0.2^{* *}$ \\
KDSGB24 & $28.6 \pm 0.1^{* *}$ & $19.2 \pm 0.3$ & $18.1 \pm 0.5$ & $66.3 \pm 0.4$ & $76.1 \pm 0.2^{*}$ & $16.0 \pm 0.2$ \\
UT-amf & $17.0 \pm 0.4$ & $4.3 \pm 0.4$ & $11.8 \pm 0.8$ & $73.6 \pm 0.1$ & $86.3 \pm 0.0$ & $15.6 \pm 0.4$ \\
amfGB22 & $23.9 \pm 0.1^{* * *}$ & $3.5 \pm 0.3$ & $13.3 \pm 1.0$ & $73.1 \pm 0.1$ & $84.7 \pm 0.1^{* *}$ & $16.0 \pm 0.2$ \\
amfGB24 & $21.8 \pm 0.2^{* * *}$ & $4.1 \pm 0.2$ & $14.0 \pm 0.6$ & $73.3 \pm 0.3$ & $85.2 \pm 0.6$ & $16.2 \pm 0.7$ \\
amfGB25 & $21.6 \pm 0.1^{* * *}$ & $4.2 \pm 0.1$ & $13.5 \pm 0.7$ & $73.7 \pm 0.3$ & $86.5 \pm 0.6$ & $18.5 \pm 0.7^{*}$ \\
amfSGB1 & $20.6 \pm 0.0^{* *}$ & $4.3 \pm 0.2$ & $16.7 \pm 0.4^{*}$ & $72.7 \pm 0.0^{* *}$ & $83.7 \pm 0.1^{* *}$ & $15.1 \pm 0.4$ \\
amfSGB4 & $20.4 \pm 0.2^{* *}$ & $3.8 \pm 0.0$ & $13.6 \pm 0.2$ & $73.4 \pm 0.1$ & $85.3 \pm 0.3$ & $15.0 \pm 0.1$ \\
amfSGB12 & $21.6 \pm 0.1^{* * *}$ & $4.1 \pm 0.1$ & $19.7 \pm 0.4^{* *}$ & $74.0 \pm 0.2$ & $86.6 \pm 0.3$ & $15.6 \pm 0.3$ \\
amfSGB18 & $23.4 \pm 0.5^{* * *}$ & $4.1 \pm 0.0$ & $26.7 \pm 2.0^{*}$ & $74.5 \pm 0.1^{*}$ & $86.4 \pm 0.3$ & $16.4 \pm 0.1$ \\
\hline
\end{tabular}

Data (mean \pm S.D.) are the average of two or three independent measurements. $P$, total phosphate content; $\mathrm{AM}$, apparent amylose content; d50, median granule size; $\mathrm{T}_{0}$ and $\mathrm{T}_{\mathrm{c}^{\prime}}$ starch gelatinization temperature; $\Delta H$, gelatinization enthalpy. Statistical significances between each starch sample and the control determined by using t-test $\left({ }^{*}, p<0.1 ;{ }^{* *}, p<0.01 ;{ }^{* * *}, p<0.001\right)$.

\section{Modified starches from the KD background exhibit a higher digestibility}

To investigate whether the starch digestibility was affected by the introduction of the (engineered) GTFB, starches from all transformants and corresponding controls were treated with a-amylase and amyloglucosidase, and the amount of released glucose was measured. Overall, a significant difference in the glucose release was observed only for the starches from the KD background relative to the control UT-KD (ANOVA, $p<0.05$ ), whereas no changes were found between starches from the amf background and the control UTamf. As shown in Fig. 6a, for the KDGB starches, the amount of released glucose ranged from 19 to $29 \mu \mathrm{g} / \mathrm{mg}$ starch, and the average value ( 23 $\mu \mathrm{g} / \mathrm{mg}$ starch) was significantly higher than that of UT-KD ( 19 $\mu \mathrm{g} / \mathrm{mg}$ starch). Moreover, $\sim 19 \%$ and $\sim 44 \%$ more glucose, in average, was released from KDSGB starches than from KDGB starches and the control, 
respectively. Starch samples of H-expressors from KD background released significantly higher amount of glucose compared to the control UT-KD (t-test, $p<0.05,0.01$ or 0.001 ), whereas there was no consistent differences in glucose release between starches from $\mathrm{H}$-expressors and control in amf background (Fig. 6b). Collectively, these results indicated that modified starches from KD background are more susceptible to degrading enzymes than the control.

(a)

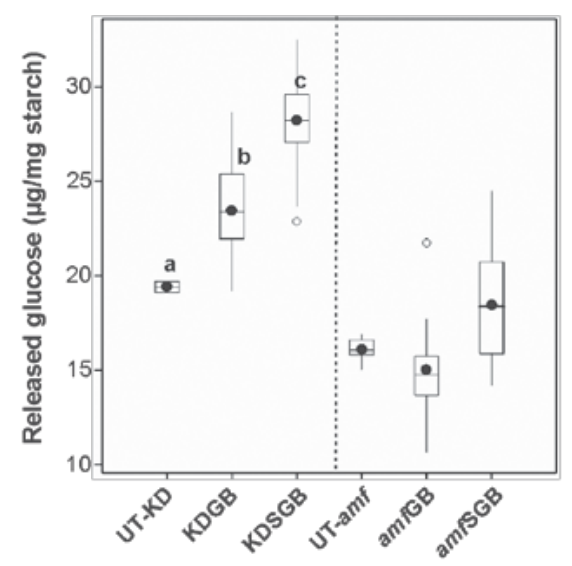

(b)

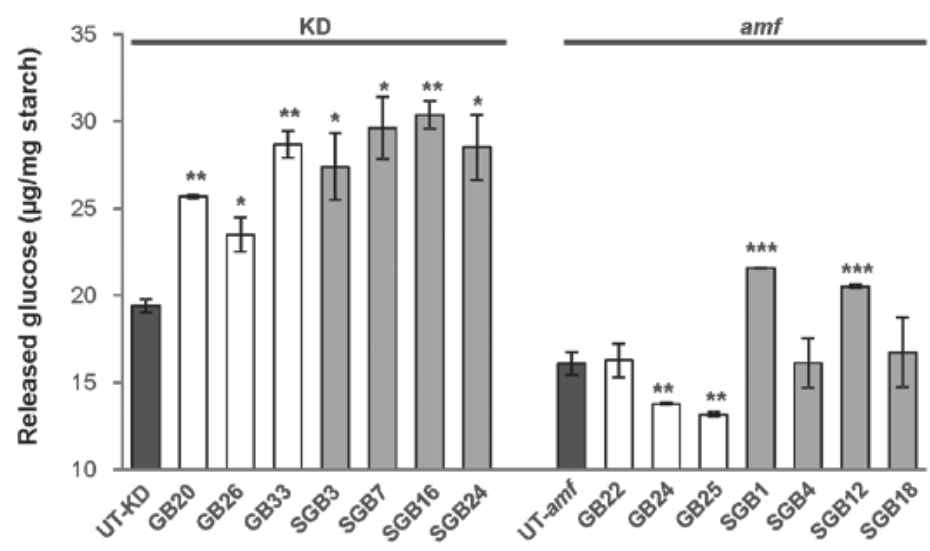

FIGURE 6. Starch digestibility analysis of starches from transformants and control lines. (a) Boxplot presenting the amount of glucose released from modified starches and their respective controls (UT-KD and UT-amf). Each starch sample was treated with a-amylase and amyloglucosidase and measured glucose amount in duplicate. The measurements were performed on starches from all transformants except $\mathrm{N}$-expressors (lines with undetectable expression of target gene), which are 31, 23, 25 and 17 lines for KDGB, KDSGB, amfGB and amfSGB series, respectively. Boxes in the plot include values in the $25 \%-75 \%$ interval. Internal lines, filled circles, unfilled circles and bars represent the median, the mean, outliers and extremes, respectively. Statistical significance was analysed using one-way ANOVA. Different letters indicate statistically significant differences between means at $p<0.05$. (b) The amount of glucose released from starches of $\mathrm{H}$-expressors (lines with high expression of target gene) from each transgenic series and control lines. Columns represent mean \pm SD from two independent measurements. Statistical significances between each starch sample and the control determined by using t-test $\left({ }^{*}, p<0.1 ;{ }^{* *}, p<0.01 ;{ }^{* * *}, p<0.001\right)$. 


\section{Genes involved in starch metabolism are up-regulated in transformants}

Expression of the (engineered) GTFB in potato leads to alterations in starch granule morphology and an increase in granule size and starch phosphate content in amf transformants. To better understand these phenomena, the transcripts of key genes involved in starch metabolism were further investigate by qRT-PCR in H-expressors from each transgenic series and respective control tubers. Generally, regardless of the constructs, amf transformants showed a significant increase (ANOVA, $p<0.01$ or 0.05 ) in the expression level of glucan, water-dikinase (GWD1), starch phosphorylase (SP), isoamylase (ISA1 and ISA2), starch branching enzyme I (SBEI) and starch synthase (SSIII) relative to the control, while no significant changes were observed for the other genes, such as $\beta$-amylase (BAM), isoamylase (ISA3), starch branching enzyme II (SBEII) and ADP-glucose pyrophosphorylase (AGPase) (Fig. 7a). Particularly, the up-regulation of gene expression was generally higher in SGB transformants than in GB transfomants. For instance, for the GWD1 expression in amfSGB15 line showed an 8-fold increase compared to the control, while in amfGB24 the expression level was increased 3-fold For the SP expression, 5- and 2-fold increases were observed in these two lines respectively. On the other hand, in the KD background, no significant changes were found in the expression of these genes between transformants and the control, except for SP (Fig. 7b). Taken together, introduction of the (engineered) GTFB into amf potatoes results in up-regulation of certain genes involved in starch metabolism, especially SGB. The same trends were found in KD background but the differences were smaller. 


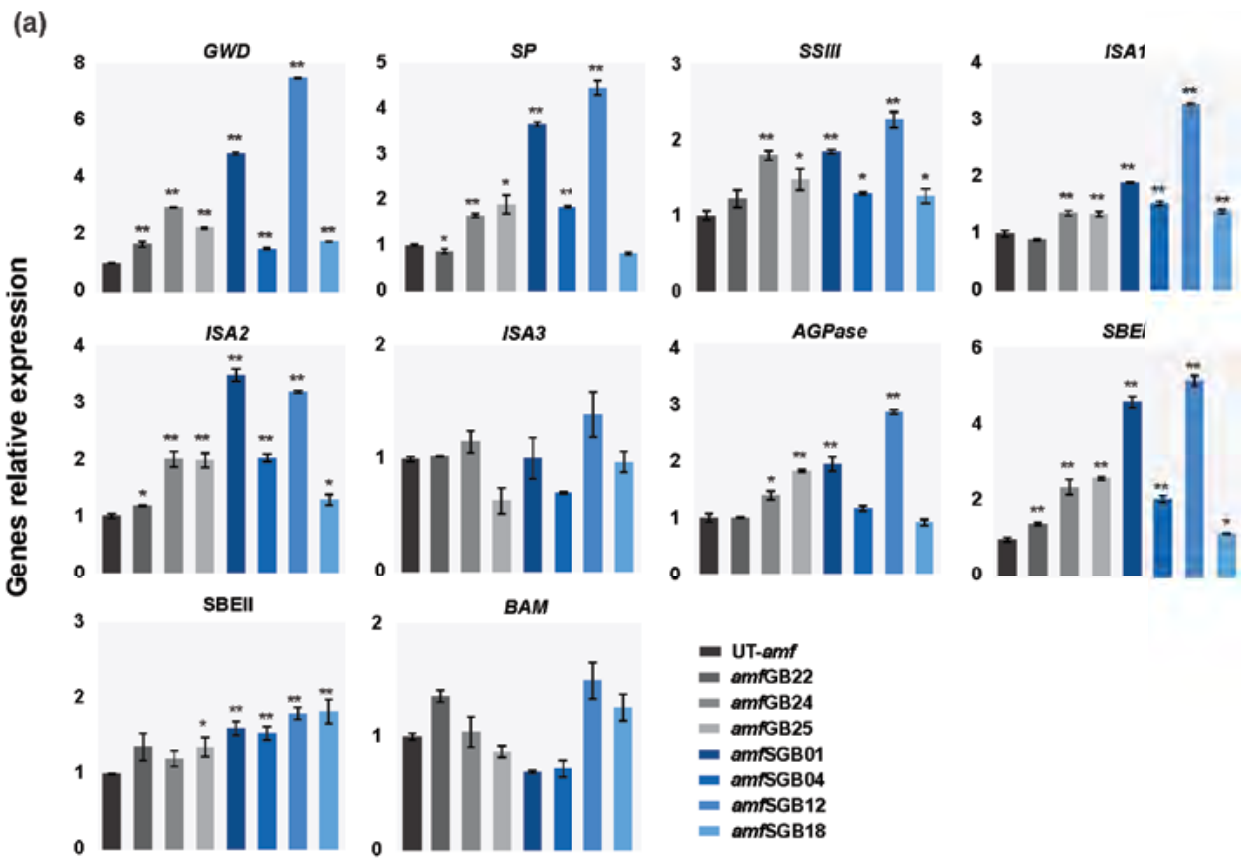

(b)

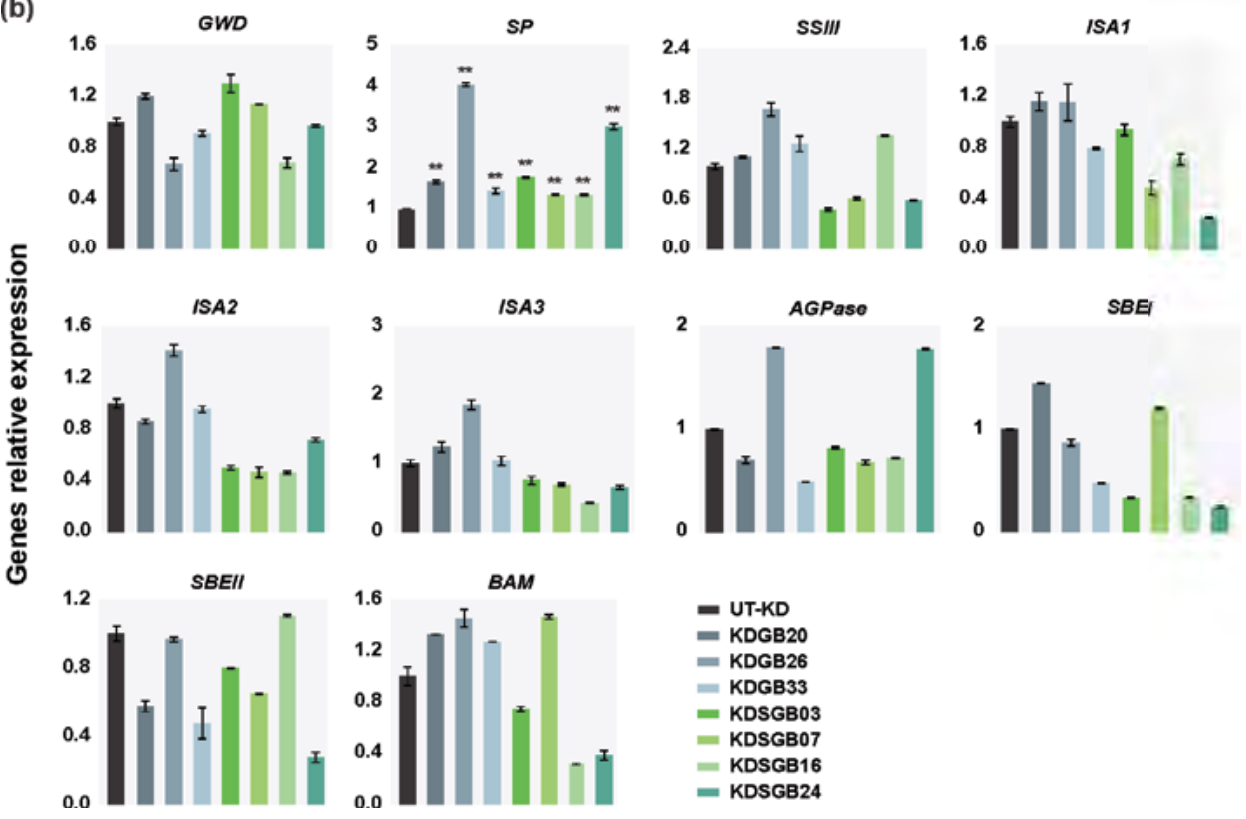

FIGURE 7. The expression level of the genes encoding key enzymes involved in starch metabolism in (a) amf and (b) KD potatoes. Include: glucan, water-dikinase (GWD1), starch phosphorylase (SP), isoamylase (ISA1, ISA2 and ISA3), starch branching genes (SBEI and SBEII), starch synthase (SSIII), $\beta$-amylase (BAM) and ADP-glucose pyrophosphorylase (AGPase). Three/Four $\mathrm{H}$-expressors (lines with high expression of target gene) of each series and the control lines were selected and subjected to qRT-PCR analysis. Statistical significance was analysed using t-test $\left({ }^{*} P<0.05 ;{ }^{* *} P<0.01\right)$. The values are expressed as the mean \pm SD from three independent measurements. 


\section{Discussion}

In this study, an (engineered) GTFB gene from Lactobacillus reuteri 121 was introduced into $\mathrm{KD}$ and amf potato plants, aiming to create liner a-1, 6-glycosidic linkages in amylopectin chains during starch biosynthesis, thus producing starches with novel structure and properties.

The results presented herein showed that differences in starch structure between modified and control starches could not be detected, suggesting that expression of the (engineered) GTFB in potatoes either does not introduce new chains with liner $a-1$, 6-glycosidic linkages into starches, or these are below detectable levels, or novel chains have been identified as an error and removed by endogenous hydrolases. The latter explanation is more likely. In other words, (engineered) GTFB could possibly introduce novel chains into amylopectin structure, while endogenous enzymes cleave off these unusual branches, leading ultimately to no changes in starch structure. Firstly, this is supported by the increase in the transcripts of starch-degrading genes (GWD1, ISA1, ISA2 and SP) and starch-synthesizing genes (SSIII, SBEI and SBEII) in transformants. Particularly, previous studies demonstrated that the removal of misplaced branches by ISA 1 and ISA2 is essential for crystalline amylopectin synthesis (Streb et al., 2008; Wu et al., 2013), which can explain the up-regulation in transcripts of these two genes in this study. This hypothesis is in line with the model proposed by Huang et al. (2013), who speculated that debranching enzymes and $\beta$-amylase remove the extra positioned branches introduced by E.coli glycogen-branching enzyme. On the other hand, while starch-degrading genes remove "error" chains, the transcripts of starch-synthesizing genes are up-regulated, thereby stimulating amylopectin synthesis for a regular granule packing.

Secondly, this hypothesis is also supported by the altered starch granule morphology from transformants, especially the uneven distribution of the growth rings, which might indicate that (engineered) GTFB introduced novel chains at first, thereby affecting the process of amylopectin assembly. Taken together, the fact that no detectable changes in starch structure via (engineered) GTFB could be observed might be due to the molecular regulation existing for starch biosynthesis.

Furthermore, a higher digestibility in modified starches in KD background compared with the control has been detected. The increase may be attributed to the irregular bumpy surface of starches that provides more surface area for the binding of hydrolytic enzymes and ultimately gives rise to more hydrolysis than control starches. This result is consistent with data obtained in earlier studies, reporting that starches with a bigger granule size and smoother granule surface have lower susceptibility to enzymatic hydrolysis (Capriles et al., 2008; Kaur et al., 2007; Lindeboom et al., 2004; Tester et al., 2006). 
On the other hand, microscopy analyses showed varied-sized pores on the surface of starch granules from SGB transformants, which can be explained by the specific granulebinding ability of SGB fusion proteins and the deposition of them on the granule surface. The surface-attached proteins might be released later on, consequently generating the porous surface. This is further supported by the observation that the percentage of altered granules increases in transgenic plants with higher SGB expression (Fig. 3b). This result is consistent with previous studies showing that surface pores were visible after removing the protein from granule surface in cereals (Kim and Huber, 2008; Naguleswaran et al., 2011). Moreover, the presence of amylose might explain the shallower pores in KDSGB starches compared to that in amfSGB. Similar observations have been made by introducing other granule-bound proteins into potatoes (Firouzabadi et al., 2007; Huang et al., 2013; Huang et al., 2014).

In the amf background, an increase in phosphate content of modified starches is likely to be a result of the up-regulation in GWD1 expression, which has proven to be mainly responsible for the starch phosphate content (Blennow et al., 2002; Lorberth et al., 1998). In addition, one could expect to observe the differences in starch composition or properties because of the changes in granule morphology, granule size and phosphate content in modified starches. However, no significant changes in amylose/amylopectin ratio, gelatinization temperatures or starch digestibility were detected between modified starches and the control starch. This phenomenon can be a consequence of multiple combined effects caused by all these alterations, each of which is difficult to quantify.

In summary, this study gives further insights into the complex functions and interplay between starch metabolic enzymes in storage starches, highlighting the molecular regulation of amylopectin assembly, and providing a further step toward understanding the biosynthesis of storage starches. Further investigation is required to decipher the precise mechanism of starch granule formation and to identify determinants that are responsible for this process. Ultimately, our results re-inforce the notion that genetic modification is not only a powerful tool for uncovering and exploiting starch biogenesis in plants, but also an effective and economical manner for tailoring starches with improved properties for industry applications like in this case the enhanced release of glucose from KD starches. 


\section{Supplementary data}

pBIN19/SBD2-Xbal

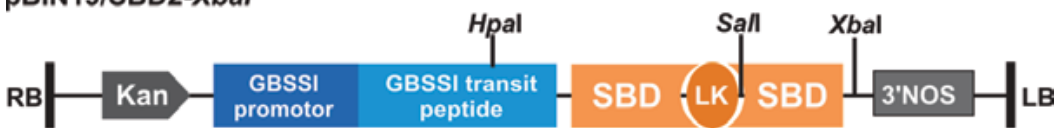

FIGURE S1. Schematic representation of pBIN19/SBD2-Xbal vector. This construct was modified based on pBIN19/SBD2 (Ji et al., 2004) by adding an Xbal restriction site. RB and LB represent right and left borders, respectively. SBD, LK, Kan and $3^{\prime} \mathrm{NOS}$ stand for starch binding domain of cyclodextrin glycosyltransferase from $B$. circulans, linker, kanamycin resistant gene and NOS terminator, respectively. Hpal, Sall and Xbal are restriction enzymes.

TABLE S1. The qRT-PCR primer sequences of genes of interest and reference gene.

\begin{tabular}{lllll}
\hline Gene & Gene ID & Forward primer 5'-3' & Reverse primer 5'-3' & Reference \\
\hline GWD1 & AY027522.1 & gggtggtctttgcgtgatt & tgtagcccatgcagtttgca & Chen et al. (2012) \\
BAM & XM_006340834.2 & cctccttccccactgatctg & gaaacgtcggtgattcctgc & Chen et al. (2012) \\
AGPase & X55155 & gctgggacccgactttatcc & cgggaatgtcaatcagacgat & Muller-Rober et al. (1990) \\
SP & X52385 & caggaaccagatgctgctctt & catagccccatgctgggtagt & Zhang et al. (2013) \\
ISA1 & 102577466 & ggcaaatggagaggacaaca & atgggaacaccttgggaaac & Bustos et al. (2004b) \\
ISA2 & 102577931 & ttatccttccgccacctc & cttcaactggagttcccttct & Bustos et al. (2004b) \\
ISA3 & 102577824 & gacgcttgcccttcattc & ctcctgtgcggttcttctgt & Ferreira (2011) \\
SBEI & 102596498 & ccgagccccacgaatctat & ggctcagagctgctcatgc & Safford et al. (1998a) \\
SBEII & 102590711 & actcgcaactgatgtggatg & ggcattccgctaacatcttc & Jobling et al. (1999) \\
SSSIII & 102577674 & cacaggaggtgtctggaaacc & tggaacttgtgaaggtgaggc & Abel et al. (1996) \\
EF1a & AB061263 & attggaaacggatatgctcca & tccttacctgaacgcctgtca & Nicot et al. (2005) \\
\hline
\end{tabular}





\section{Chapter 7}

General Discussion 
Starch, the major storage carbohydrate, provides energy for plants during heterotrophic growth, and is essential for plant survival. It is also a versatile biopolymer, serving as staple foodstuffs in human diets and as a renewable raw material for a wide range of industrial applications (Jobling, 2004). The main reasons for the wide use of starch are the fact that it is relatively cheap, abundant and biodegradable. Another important reason related to the physico-chemical properties is that it can be (easily) modified to meet specific industry's needs. However, derivatized starches produced by chemical, physical and enzymatic modifications face a big challenge, as they do not meet environmental impact demands. A promising strategy to overcome this challenge entails the production of modified starch in planta using genetic modification, which is attracting increasingly attention among scientists as a possible "green" alternative to enhance starch functionalities. At its core, this strategy requires a thorough understanding of the structure, composition and synthesis of starch, and the determinants of starch structural characteristics and properties. In this regard, much progress has been made in studying transitory starch due to the wealth of genetic and genomic resources in Arabidopsis thaliana, which could provide key leads for elucidating starch metabolism in economically important storage organs.

Potato starch has superior processing amenability and unique properties relative to many other starches; therefore, producing potato starches with improved properties is of great industrial interest. Additionally, potato is an outstanding model for studying storage starch metabolism due to the large number of genomic and molecular tools available, such as a sequenced genome and well established transformation protocols.

In this thesis, to produce unconventional potato starch with novel properties using genetic modification, attempts have been made from two perspectives: one is to modulate the phosphate content of starch (Chapters 3, 4 and 5); another is to create novel polymers in starch (Chapter 6). To this end, a number of enzymes from various sources have been expressed or modulated in potato tubers, followed by the comprehensive and systematic characterization of starch structure and properties and a thorough analysis of the expression of genes involved in starch biosynthesis. In the following sections, the results of the individual experiments, especially the implications of modifications of phosphate content on starch properties and starch metabolism, are compared and discussed. At the end, future prospects for starch bioengineering and potential strategies are appraised. 


\section{Starch biosynthesis seems to be under strict regulation, thereby limiting the effect of genetic modification}

\section{The phosphate content of potato starch is strongly controlled}

Production of phosphorylated starch directly in the crop has great advantages both economic and for the environment. Many efforts have been made in various crops to alter starch phosphate content with focus on modulating the expression of the glucan, water dikinase (GWD) gene, which is mainly responsible for phosphorylating starch (Carciofi et al., 2011; Frohberg et al., 2013; Lanahan and Basu, 2004; Lorberth et al., 1998; Schewe et al., 2002; Schwall et al., 2000). Meanwhile, starch phosphatases have also emerged as crucial enzymes in starch metabolism in more recent years (Emanuelle et al., 2016). It was demonstrated that starch phosphate content is influenced by both starch phosphorylating enzymes and phosphatases, representing the net outcome of the combinatorial activity of both types of enzymes. Thus, manipulating starch phosphatases may also have great potential to alter the phosphate content of starch.

To this end, several enzymes related to (de)phosphorylation of starch were expressed or modulated in potato tubers, including phosphatase laforin from human (Chapter 4), a glucan, water dikinase (GWD1) from potato (Chapter 3) and glucan, water dikinase 2 and 3 (AtGWD2 and AtGWD3) from Arabidopsis thaliana (Chapter 5). Results generated from all these independent experiments strongly suggest that the phosphate content in potato starch is under stringent control.

Firstly, we showed that modified starch from transgenic plants carrying the (engineered) laforin expression exhibited a considerably increase in phosphate content, rather than the expected decrease (Fig. 5, Chapter 3). A key discovery to underpin this result is the observation regarding the elevation in the expression of starch phosphorylating genes. It can therefore be postulated that there is a compensatory response to the loss of phosphate content via upregulation of genes encoding starch dikinases during starch biosynthesis, probably to ensure the maintenance of the appropriate proportion of phosphate esters in starch granules (Chapter 3).

Another interesting finding emerging from these studies is the existence of a threshold level of phosphate content in potato starch containing wild-type-like structure and composition. Over the last decades, highly phosphorylated starch has been achieved in wheat (Schewe et al., 2002), maize (LanahanMichael and Ship, 2005), rice (Frohberg et al., 2008) and barley (Carciofi et al., 2011) by only overexpressing GWD1. However, in our study, overexpression of GWD1 in potato tuber resulted in only about $20 \%$ increase in phosphate content in both genetic backgrounds, which is a smaller increase compared to the other crops (Fig. 2, Chapter 4). Likewise, a comparable amount of the increase in starch 
phosphate was found in modified starch from transgenic plants carrying the (engineered) laforin expression (Chapter 3). Additionally, expression of GWD2 and GWD3 in potato tubers did not result in a significant increase in starch phosphate content (Chapter 5). Taken together, the presence of a threshold level of phosphate content raises an interesting question as to whether it is possible to largely increase the phosphate content of potato starch, given that potato starch is already naturally highly phosphorylated compared to other (cereal) starches. It seems relevant to recognize that there are limiting factors for the incorporation of phosphate esters into starch granules rather than the phosphorylating enzymes themselves.

In fact, increasing phosphate content in potato starch has been achieved in earlier studies, but only as an indirect effect of engineering of other enzymes in starch biosynthesis, such as suppression of starch branching enzymes (Schwall et al., 2000) or starch synthases (Abel et al., 1996; Jobling et al., 2002). In our study, we have also observed a higher increase in phosphate content of starches containing lower amylose content as compared to the control (Chapter 3). The reason for this effect is supposedly that the alteration in starch structure and composition resulted in a better substrate (e.g. long amylopectin chain, more amylopectin) for GWD1, hence stimulating GWD1 activity and consequently adding more phosphate esters in starch granules (Blennow, 2015; Mikkelsen et al., 2004). Collectively, starch structure and composition are proposed as possible factors regulating the proportional incorporation of phosphate content into starch granules. Furthermore, we suggest that engineering potato starch for an even higher phosphate content is only possible if more space is available for the deposition of phosphate esters resulting from an alteration in the structure or amylose/amylopectin ratio of starch. Further attempts should therefore be directed towards increasing amylopectin content with longer chains in starch granules, while simultaneously upregulating the expression of starch phosphorylating genes. Prospects for such endeavors seem reasonably feasible given successful illustrations of simultaneous manipulation of key genes involved in starch biosynthesis in potato (Edwards et al., 1999; Jobling et al., 2002).

\section{Self-repair ability for optimizing starch structure}

The structure of starch varies substantially among the botanical sources, which has since long been recognized as one of the key features that influences starch functional properties (Delgado-Escueta et al., 1999; Edwards et al., 1999; Jobling, 2004). Notwithstanding continuous efforts in the past decades, starch structure, as an appealing target for engineering starch with novel functionalities, has not been modified effectively via modulating endogenous enzymes in planta so far (Ball and Morell, 2003; Blauth et al., 2002; Jobling, 2004; Safford et al., 1998b; Satoh et al., 2003). This is not surprising since starch structure is manufactured in a delicate and complex way through the orchestrated 
action of many enzymes, including starch synthases, branching and debranching enzymes (Chapters 1 and 2). In particular, the latter is the key determinant of the structure by liberating misplaced branches to permit the efficient and accurate packing of starch granules.

The presence of this self-repair mechanism is again confirmed in our study through the transcript analysis of transgenic tubers carrying the (engineered) 4, 6-a-glucanotransferase (GTFB) from Lactobacillus reuteri 121 (Chapter 6). The results demonstrated that the effect of the target enzyme, creating new chains with liner a-1, 6-glycosidic linkages, was diminished by the action of starch debranching enzymes while cooperating with other starch degrading enzymes. Along a similar line of thought, earlier studies have attempted to diversify starch structure by incorporating novel types of linkages; however, no significant alteration in starch structure was detected (Huang et al., 2013; Kok-Jacon et al., 2005a; Kok-Jacon et al., 2007; Kok-Jacon et al., 2005b; Kok-Jacon et al., 2005c). In one of these studies, the authors also observed upregulation of genes involved in starch degradation, which is in line with our result and hypothesis. Collectively considered, it would be very interesting to investigate whether stronger changes in the starch structure can be generated in the plants lacking starch debranching enzymes, thereby shedding more light on the nature of this self-repair process. Elucidating this mechanism not only helps us gain better insights into the factors governing starch structure and metabolism, but may also pave a way for tailoring starch with novel structure in planta.

\section{Other stumbling blocks in the way of genetic modification of starch}

It is well known that starch is very complex in structure, but relatively simple in chemical composition (i.e. only glucose units). This chemical simplicity prevents us from obtaining the definitive structural information, hindering the description of the actual structure and detection of alteration in starch structure (Pfister and Zeeman, 2016). Additionally, the current analytical methods for characterizing structural features (e.g. chain length distribution and the frequency of branching) give average measurements, concealing the heterogeneity of starch structure. On one hand, this limitation makes it difficult to examine the substrate preferences of enzymes or mutant phenotypes in relation to structure alteration, thereby impeding the interpretation of the function of specific enzymes. On the other hand, this limitation generates obstacles for detecting the novel structure introduced during starch biosynthesis. For instance, if a low amount of linear a-1, 6-glycosidic linkages is present in starch structure (Chapter 6), we may not be able to detect them.

On the other hand, another stumbling block is the existence of complex interplay between biosynthetic enzymes, as the alteration in the activity of one enzyme can lead to changes in the substrate of other enzymes, and vice versa. These pleiotropic effects make it difficult 
to investigate, for instance, the individual effect of each gene on structural changes, or, the effect of different starch components or structural features on starch functional properties. For this reason, to date the relationship between starch characteristics and physico-chemical properties remains unclear, which slows down the development of tailored starches.

\section{Self-repair mechanisms of starch biosynthesis make sense}

As described above, the phosphate content and starch structure of potato starch are clearly subject to complex control, however, the underlying reasons for these phenomena are unclear. Here, the crucial role of phosphate esters in starch structure during starch biosynthesis are presented and discussed, aiming at understanding the biological meaning of these self-repair mechanisms, including compensatory mechanism for regulating starch phosphate content and self-repair ability for optimizing starch structure.

\section{Regularity of chain structure and appropriate amounts of phosphate esters are prerequisites for the normal assembly of a starch granule}

Granules present a high-ordered and stable form of storing starch in plants, presumably awarding an evolutionary and physiological advantage over the accumulation of soluble carbohydrates (Streb and Zeeman, 2012). The assembly of the starch granule is an intricate process involving many developmental steps (Chapter 1). One of the key steps is building up a framework of the crystalline lamellae resulting from a high-density packing of double helices formed by the neighboring chains of amylopectin. Logically, it is easy to envisage that any changes in the types of linkages and branching structure of amylopectin can result in a deleterious change in the packing of double helices and hence the crystalline structure of starch granules. In potato, this disturbance in starch structure could affect the starch formation thus the growth processes upon sprouting, as starch serves as the longterm energy source. Therefore, the delicate regulations of starch biosynthesis are required for impeccable assembly of granules and subsequently for plant survival and growth. For instance, the removal of "error" chains is governed by such self-repair mechanism, thus ensuring the regularity of chain structure and consequently the normal assembly of starch granules (Chapter 6).

In addition, the molecular force field model (Blennow, 2015; Blennow et al., 2002) showed that the incorporation of phosphate esters at the $\mathrm{C}-3$ position of the glucose unit in starch affects the stability of double helices, whereas phosphate esters at the C- 6 position can induce hydration between the helical arrays to distort helix-helix interactions. To this extent, we propose that the constraint on the maximal level of phosphate content is 
necessary and this compositional threshold should not be surpassed, since the amount of phosphate esters is essential to form a correct granule structure (Chapter 3, 4 and 5). On the other hand, we showed that starch granules containing extremely low phosphate content exhibited severe fissures; however, these fissures were diminished with increasing phosphate content and ultimately eliminated in starch granules with phosphate content higher than $\sim 15 \mathrm{nmol} / \mathrm{mg}$ starch. Interestingly, this defective morphology was not observed in the amylose-free starch granules (Chapter 4). These findings, together with the fact that phosphate esters are mainly bound to amylopectin chains, allow us to conclude that phosphate esters serve as identifiers of amylose and amylopectin chains thus preventing the interaction between these two types of chains and permitting the normal assembly of granules [Chapter 4, (Blennow et al., 2002)]. Therefore, a minimal amount of phosphate content is required for the normal assembly of starch granules if amylose molecules are present. This is possibly one of the reasons why potato plants endeavor to compensate the loss of phosphate content in starch granules (Chapter 3 ).

\section{Starch phosphorylation, an indispensable piece for starch metabolism}

Starch phosphorylation confers starch granules with the capability for physical amorphisation, solubilisation and ultimate amylolytic degradation, regulating mobilization of energy reserves on metabolic demands (Blennow, 2015). Suppression of starch phosphorylation by knock-down or knock-out of GWDs leads to starch excess phenotypes in leaves and cold-sweetening inhibition in potato tubers, indicating the essential role of starch phosphorylation in starch-degrading machinery (Blennow et al., 2003; Kozlov et al., 2007; Lorberth et al., 1998; Mahlow et al., 2014; Yu et al., 2001b). In our study, the association between starch phosphorylation and starch degradation was reflected in the simultaneous change in transcript level of GWDs and that of other degrading genes (Chapters 3, 4 and 6), which re-enforces the effect of starch phosphorylation on starch degradation. The suggested mechanistic explanation of this phenomenon is that phosphate esters disrupt the crystalline structure of amylopectin and by that facilitate the access of degrading enzymes (Blennow, 2015; Zeeman et al., 2010).

In contrast, the effect of starch phosphorylation on the process of starch synthesis is elusive. Nonetheless, the ubiquity of phosphate esters in various starches strongly indicates a requirement for a pre-phosphorylation during the synthesis of starch. As explained in the previous section, phosphate esters have the capability to modulate the crystallinity of starch, which is indispensable for the efficient packing of well-structured granules. Understandably, the corporation of phosphate esters during starch synthesis results in a build-in starch degradability, which can prepare starch granules for dissolution before starch degradation starts, thus executing an energy-efficient degradation. Surprisingly, suppression of GWD1 resulted in unaltered starch content in various organs, including 
Arabidopsis thaliana (Skeffington et al., 2014), wheat endosperm (Ral et al., 2012) and potato tuber (Chapter 4), indicating that starch phosphorylation may affect not only starch degradation but also starch synthesis. Studies on GWD-deficient arabidopsis mutants demonstrated the existence of an inhibition of starch synthesis in leaves (Skeffington et al., 2014). This is in line with the results of gene expression analysis in potato tubers lacking GWD1, which indicates that starch phosphorylation affects starch synthesis by controlling the carbon flux into starch while simultaneously modulating starch-synthesizing genes (Chapter 4). Further studies on protein and metabolic level are needed to confirm this finding.

In summary, phosphate esters and chain structure to a large extent delineate the architectural characteristics of starch granules and fundamentally influences plant primary metabolism. Certainly, the exquisite regulations of these features are crucial in preventing extreme disruption in the inherent compositional balance of starch and permitting plant normal growth; however, such mechanisms increase the difficulties to modify starch structure and composition and optimize the functionality of it in planta. Hence, future strategies should take into account how to suppress such self-repair mechanisms to circumvent the natural limitations of starch granules. Or, conversely, we may take advantage of this frailty to achieve an efficient and effective modification by harnessing such mechanisms and applying the principle into genetic modification; for instance, generating potato starch with high phosphate content by making use of the compensatory responses in planta.

\section{Starch morphology, a changeable characteristic}

Starch morphology is organ and species specific and is therefore presumably genetically controlled (Streb and Zeeman, 2012). In spite of the great progress in identifying enzymes that are relevant to starch biosynthesis, very little is known regarding the key factors controlling granule morphology and regulating granule formation. In this thesis, similar to previous studies (Huang et al., 2013; Huang et al., 2014; Kok-Jacon et al., 2005a; KokJacon et al., 2007; Kok-Jacon et al., 2005c; Xu et al., 2014), expression of heterologous genes in the potato tubers resulted in severe alterations in starch granule morphology (Chapters 3, 5 and 6). We have demonstrated that starch chemical composition, including proteins, amylose molecules and phosphate esters, plays important roles in starch granule morphology.

Generally, we found that modified starches showed irregular shapes relative to the controls, regardless of the genetic background and gene expressed. These alterations can be the consequence of depositing newly introduced proteins in the starch granules or/and changing the activity of endogenous enzymes involved in the starch biosynthesis. Huang 
et al. (2013) suggested that introduced protein might influence the stability of the helices or the side-by-side packing of helices and thereby interrupting the optimal crystallization of starch granules. This may also partly explain the observations in our studies. However, in contrast to their findings, we observed considerable changes in the expression of a number of genes involved in the starch biosynthesis in different transgenic series, such as in the transgenic plants carrying the (engineered) laforin/GTFB (Chapters 3 and 6). These results indicated that the alteration in starch granule morphology may also be caused by the interference with the expression of endogenous genes, especially in the transgenic lines carrying introduced proteins that are not able to bind to starch granules.

On the other hand, comparison of modified starches from two genetic backgrounds (amylose-containing and amylose-free background) elucidated that amylose plays an important role in the granule morphology. To illustrate, we observed cratered surfaces in the starch granules from transgenic lines carrying starch-binding GTFB regardless of genetic background (Fig. 3, Chapter 6), which was most likely created by the temporary deposition of the protein on the granule surface. Interestingly, the craters were shallower on the surface of granules from amylose-containing background relative to those from amylose-free background. In the amylose containing background it seemed as if the craters were partially filled by amylose molecules. Moreover, the importance of amylose in determining granule morphology again became visible in the morphological alteration of starch granules from transgenic tubers with suppressed GWD1. Severe fissures were observed in the modified starch granules from amylose-containing background, but not in the ones from amylose-free background (Fig. 4, Chapter 4). Future research using a highamylose containing background is needed to further investigate the effect of amylose on starch granule morphology.

In our study, phosphate esters displayed drastic effects on the morphology of starch granules (Fig. 4, Chapter 4). Regardless of genetic background, starch granules containing extremely low phosphate content showed a much more spherical shape and more concentric rings compared with their respective controls, whereas starch granules containing high phosphate content displayed irregular bumpy shape. The molecular model of phosphorylated starch proposed by Blennow et al. (2002) demonstrated that C-3-bound phosphate esters protrude from the double-helical surface, which may have great impact on the crystalline packing of starch granules. In this regard, the removal of phosphate esters may lead to even more compact assembly of granules, resulting in a more regular ring patterns and granule shape. Additionally, as described before, phosphate esters are also responsible for the 'cracked' morphology in starch granules, since they act as identifiers of amylopectin and amylose to prevent the erroneous interactions between these two segments hence allowing the normal assembly of starch granules. 
Overall, granule morphology is a good indicator of abnormal functioning of starch biosynthesis. Although changes in many characteristics can be repaired or compensated for, errors made in earlier stages will be forever recorded in starch granules and reflected on granule morphology since growth of the granule is a continuous process. Moreover, our data illuminated that starch granule morphology is determined at least by the deposition of protein, the presence of amylose and the level of phosphate esters. It presents as the overall outcome of the combined effect of all these parameters. Certainly, the complexity of this process introduces great difficulties in exploiting the key players and the underlying mechanism. Considerably more work will be needed to dissect the effect of each starch component on the alteration of granule morphology and ultimately to unveil the mechanism of controlling starch granule morphology.

\section{Starch modification requires a thorough understanding of the effects of each starch feature on each specific property}

The diversity in the functional properties of various starches is largely attributed to the substantial differences in their characteristics such as starch composition, structure, granule morphology and size, etc. A thorough investigation on the properties of modified starches from the same species (even same genetic background) can better explore the effect of each characteristic by eliminating the extra variations introduced by different species. To this end, in this study, the properties of all modified starches have been tested with the focus on gelatinization characteristics, gel freeze-thaw stability and starch digestibility (Chapters 3, 4, 5 and 6).

Gelatinization characteristics influence starch processing and hence determine the suitability of starch for particular industrial applications. Amylose/amylopectin ratio and level of phosphate esters are known to strongly affect the gelatinization process (Kozlov et al., 2007). To investigate the impact of these two components on starch properties, two series of modified starches generated in this study were particular interesting. One series is composed of starches containing different levels of amylose (Chapter 3), while another series is made up of starches with a wide range of phosphate content (Chapter 4). In line with previous research (Kozlov et al., 2007), gelatinization temperatures increased in modified starches containing low amylose content; however, the gelatinization enthalpy did not show the expected increase. We reason that the effect of the decreased amylose content on gelatinization enthalpy might be counteracted by the opposite effect brought by the increased phosphate content in these starches (Chapter 3). On the other hand, a significant decrease was observed in gelatinization enthalpy of modified starches containing normal amylose content and low phosphate content. This is certainly an effect of the suboptimal crystalline structure resulting from the loss of phosphate content or/ and alterations in starch fine structure (Chapter 4). 
A better understanding of the digestibility of starch is important given the increasing interest on diet-related health complications, particularly obesity. In this study, the crucial role of surface characteristics in digesting starch was found, as the granules with bumpy surface can provide bigger surface area for being easily attacked by amylase (Chapter 6). However, like other properties, more starch characteristics are involved in controlling starch digestibility, such as phosphate content (Absar et al., 2009) and starch structure (Syahariza et al., 2013). Surprisingly, no significant change in the starch digestibility among starches containing different levels of phosphate content was observed (Chapter 4), indicating that the effect may be offset by the effect of other starch features, including granule surface characteristics, fine structure of amylopectin and amylose contents. In contrast, in spite of the changes in the other starch characteristics, phosphate content exhibited a strong dominant role in the freeze-thaw stability of starch gels (Chapter 4).

In conclusion, optimization of starch functional properties requires a clear understanding of the role of each starch characteristic (whether collectively or independently) on starch properties and changes in starch characteristics correspond to the observed functional properties. However, the one-to-one correlation between each starch characteristic and property established from each independent study does not represent a general correlation, since each starch property is an outcome of the combined effect of many characteristics and highly depends on the genetic background. Therefore, the interaction effect between multiple factors should be taken into account when designing starches with desired properties.

\section{Future perspective - "green" and socially-acceptable approach is required for starch bioengineering}

Starch as a multifunctional raw material has tremendous potential for the realization of a robust bio-based circular economy to overcome the challenge facing rapid climate change and population growth. In the past decades, advances in the genetic modification of starch have lightened up the path to produce starches with enhanced functionalities in an environmental friendly and economic manner. Nonetheless, controversies and public concern surrounding genetically modified crops give rise to strict regulatory frameworks governing the cultivation, trade and use of transgenic crops, which greatly hampers the development and commercialization of novel starches from transgenic crops. With the rapid development of biotechnology, it is envisioned that socially-acceptable tools for starch bioengineering will emerge in the near future to accelerate the commercialization process of novel starches.

Finally, continuous endeavors need to be made for identifying key genes controlling starch characteristics and exploiting the underlying mechanisms. In this respect, studies 
in the model plant Arabidopsis thaliana have greatly enhanced our understanding; however, the knowledge gained from those studies cannot be fully applied to crop plants for modifying storage starch [(Zeeman et al., 2010), this thesis]. Therefore, a better understanding of starch biosynthesis and key players in crop plants is necessary. To achieve this, identification of genetic marker associated with starch characteristics in crops (Werij et al., 2012) and new mutations in TILLING populations (Slade et al., 2012) provide tremendous potential gains. All in all, "green" and socially-acceptable approaches are required for starch bioengineering.

\section{Final remarks}

Potato starch, a tremendous valuable raw material, can be modified with enhanced functionalities in planta using green technology - genetic modification. However, the biosynthesis of potato starch is governed by complex and exquisite molecular regulations, which diminishes the effect of transgenes on the starch phosphate content and structure. Researchers should take into account these mechanisms in the future when attempting to efficiently tailor potato starch directly in plants. Additionally, this study also indicated that the insights obtained in the studies of Arabidopsis thaliana do not one on one apply to storage starches. A better understanding of the process of starch synthesis and degradation in storage organs would be a great step forward towards modifying starch in economically important crops as potato. In this respect, the investigation of the relationship between starch structural characteristics and starch properties is vital, as the knowledge can help to predict the functionality of modified starch with specific structural features. I firmly believe that producing novel starches with enhanced properties in an environmental-friendly, (cost-) efficient and predictive manner is imminent, along with the increasing availability of biotechnological tools and resources for crop plants. These versatile raw biomaterials will play an important role in realizing a robust bio-based circular economy, imparting significant effects on the quality of our life and helping us overcome the challenges related to a growing population and climate change. 
References

Summary

Acknowledgemetns

About the author

Education Statement 



\section{References}

Abel, G.J., Springer, F., Willmitzer, L. and Kossmann, J. (1996) Cloning and functional analysis of a cDNA encoding a novel $139 \mathrm{kDa}$ starch synthase from potato (Solanum tuberosum L.). The Plant journal : for cell and molecular biology 10, 981-991.

Absar, N., Zaidul, I., Takigawa, S., Hashimoto, N., Matsuura-Endo, C., Yamauchi, H. and Noda, T. (2009) Enzymatic hydrolysis of potato starches containing different amounts of phosphorus. Food Chemistry 112, 57-62.

Asatsuma, S., Sawada, C., Itoh, K., Okito, M., Kitajima, A. and Mitsui, T. (2005) Involvement of alpha-amylase I-1 in starch degradation in rice chloroplasts. Plant Cell Physiol 46, 858-869.

Bai, Y., van der Kaaij, R.M., Leemhuis, H., Pijning, T., van Leeuwen, S.S., Jin, Z. and Dijkhuizen, L. (2015) Biochemical Characterization of the Lactobacillus reuteri Glycoside Hydrolase Family 70 GTFB Type of 4,6-alphaGlucanotransferase Enzymes That Synthesize Soluble Dietary Starch Fibers. Appl Environ Microbiol 81, 7223-7232.

Ball, S., Guan, H.-P., James, M., Myers, A., Keeling, P., Mouille, G., Buléon, A., Colonna, P. and Preiss, J. (1996) From glycogen to amylopectin: a model for the biogenesis of the plant starch granule. Cell 86, 349-352.

Ball, S., Marianne, T., Dirick, L., Fresnoy, M., Delrue, B. and Decq, A. (1991) A Chlamydomonas-Reinhardtii LowStarch Mutant Is Defective for 3-Phosphoglycerate Activation and Orthophosphate Inhibition of AdpGlucose Pyrophosphorylase. Planta 185, 17-26.

Ball, S.G. and Morell, M.K. (2003) From bacterial glycogen to starch: Understanding the biogenesis of the plant starch granule. Annual review of plant biology 54, 207-233.

Baunsgaard, L., Lutken, H., Mikkelsen, R., Glaring, M.A., Pham, T.T. and Blennow, A. (2005) A novel isoform of glucan, water dikinase phosphorylates pre-phosphorylated alpha-glucans and is involved in starch degradation in Arabidopsis. The Plant journal: for cell and molecular biology 41, 595-605.

Bay-Smidt, A.M., Wischmann, B., Olsen, C. and Nielsen, T. (1994) Starch bound phosphate in potato as studied by a simple method for determination of organic phosphate and (31) P-NMR. Starch-Staerke (Germany).

Baysmidt, A.M., Wischmann, B., Olsen, C.E. and Nielsen, T.H. (1994) Starch Bound Phosphate in Potato as Studied by a Simple Method for Determination of Organic Phosphate and P-31-Nmr. Starch-Starke 46, 167-172.

Bhattacharyya, M.K., Smith, A.M., Ellis, T.H.N., Hedley, C. and Martin, C. (1990) The Wrinkled-Seed Character of Pea Described by Mendel Is Caused by a Transposon-Like Insertion in a Gene Encoding Starch-Branching Enzyme. Cell 60, 115-122.

Bindzus, W., Altieri, P.A., Kasica, J.J. and Trzasko, P.T. (2002) Starch phosphate ester composition, process and method of use in food. Patent US6428836.

Bird, A.R., Hayakawa, T., Marsono, Y., Gooden, J.M., Record, I.R., Correll, R.L. and Topping, D.L. (2000) Coarse brown rice increases fecal and large bowel short-chain fatty acids and starch but lowers calcium in the large bowel of pigs. J Nutr 130, 1780-1787.

Bjorn, G.-A.I. and Johan, T.G.A. (1948) Manufacture of infusion and injection fluids. Patent US2437518.

Blauth, S.L., Kim, K.-N., Klucinec, J., Shannon, J.C., Thompson, D. and Guiltinan, M. (2002) Identification of Mutator insertional mutants of starch-branching enzyme 1 (sbe1) in Zea mays L. Plant molecular biology 48, 287297.

Blennow, A. (2015) Phosphorylation of the starch granule. In: Starch pp. 399-424. Springer.

Blennow, A., Bay-Smidt, A.M., Olsen, C.E. and Moller, B.L. (2000a) The distribution of covalently bound phosphate in the starch granule in relation to starch crystallinity. International journal of biological macromolecules 27, 211-218.

Blennow, A. and Engelsen, S.B. (2010) Helix-breaking news: fighting crystalline starch energy deposits in the cell. Trends in plant science 15, 236-240.

Blennow, A., Engelsen, S.B., Munck, L. and Moller, B.L. (2000b) Starch molecular structure and phosphorylation investigated by a combined chromatographic and chemometric approach. Carbohyd Polym 41, 163174.

Blennow, A., Engelsen, S.B., Nielsen, T.H., Baunsgaard, L. and Mikkelsen, R. (2002) Starch phosphorylation: a new front line in starch research. Trends in plant science 7, 445-450. 
Blennow, A., Hansen, M., Schulz, A., Jørgensen, K., Donald, A.M. and Sanderson, J. (2003) The molecular deposition of transgenically modified starch in the starch granule as imaged by functional microscopy. Journal of Structural Biology 143, 229-241.

Blennow, A., Jensen, S.L., Shaik, S.S., Skryhan, K., Carciofi, M., Holm, P.B., Hebelstrup, K.H. and Tanackovic, V. (2013) Future cereal starch bioengineering: cereal ancestors encounter gene technology and designer enzymes. Cereal Chem 90, 274-287.

Brisson, N., Giroux, H., Zollinger, M., Camirand, A. and Simard, C. (1989) Maturation and subcellular compartmentation of potato starch phosphorylase. The Plant cell 1, 559-566.

Burton, R.A., Jenner, H., Carrangis, L., Fahy, B., Fincher, G.B., Hylton, C., Laurie, D.A., Parker, M., Waite, D., van Wegen, S., Verhoeven, T. and Denyer, K. (2002) Starch granule initiation and growth are altered in barley mutants that lack isoamylase activity. Plant Journal 31, 97-112.

Bustos, R., Fahy, B., Hylton, C.M., Seale, R., Nebane, N.M., Edwards, A., Martin, C. and Smith, A.M. (2004a) Starch granule initiation is controlled by a heteromultimeric isoamylase in potato tubers. Proceedings of the National Academy of Sciences 101, 2215-2220.

Bustos, R., Fahy, B., Hylton, C.M., Seale, R., Nebane, N.M., Edwards, A., Martin, C. and Smith, A.M. (2004b) Starch granule initiation is controlled by a heteromultimeric isoamylase in potato tubers. Proceedings of the National Academy of Sciences of the United States of America 101, 2215-2220.

Buttrose, M.S. (1960) Submicroscopic Development and Structure of Starch Granules in Cereal Endosperms. J Ultra Mol Struct R 4, 231-257.

Capriles, V.D., Coelho, K.D., Guerra-Matias, A.C. and Areas, J.A.G. (2008) Effects of processing methods on amaranth starch digestibility and predicted glycemic index. Journal of Food Science 73, H160-H164.

Carciofi, M., Shaik, S.S., Jensen, S.L., Blennow, A., Svensson, J.T., Vincze, É. and Hebelstrup, K.H. (2011) Hyperphosphorylation of cereal starch. Journal of Cereal Science 54, 339-346.

Caspar, T., Huber, S.C. and Somerville, C. (1985) Alterations in Growth, Photosynthesis, and Respiration in a Starchless Mutant of Arabidopsis-Thaliana (L) Deficient in Chloroplast Phosphoglucomutase Activity. Plant physiology 79, 11-17.

Caspar, T., Lin, T.P., Kakefuda, G., Benbow, L., Preiss, J. and Somerville, C. (1991) Mutants of Arabidopsis with Altered Regulation of Starch Degradation. Plant physiology 95, 1181-1188.

Ceballos, H., Sanchez, T., Morante, N., Fregene, M., Dufour, D., Smith, A.M., Denyer, K., Perez, J.C., Calle, F. and Mestres, C. (2007) Discovery of an amylose-free starch mutant in cassava (Manihot esculenta Crantz). Journal of agricultural and food chemistry 55, 7469-7476.

Chen, X., Song, B., Liu, J., Yang, J., He, T., Lin, Y., Zhang, H. and Xie, C. (2012) Modulation of gene expression in cold-induced sweetening resistant potato species Solanum berthaultii exposed to low temperature. Molecular genetics and genomics 287, 411-421.

Chen, Z., Schols, H. and Voragen, A. (2003) Starch granule size strongly determines starch noodle processing and noodle quality. Journal of food science $68,1584-1589$.

Chiu, C.-w. and Solarek, D. (2009) Modification of starches. Starch: Chemistry and technology 3, 629-655.

Comparot-Moss, S., Kotting, O., Stettler, M., Edner, C., Graf, A., Weise, S.E., Streb, S., Lue, W.L., MacLean, D., Mahlow, S., Ritte, G., Steup, M., Chen, J., Zeeman, S.C. and Smith, A.M. (2010) A putative phosphatase, LSF1, is required for normal starch turnover in Arabidopsis leaves. Plant physiology 152, 685-697.

Cote, G.L. (1992) Low-Viscosity Alpha-D-Glucan Fractions Derived from Sucrose Which Are Resistant to Enzymatic Digestion. Carbohyd Polym 19, 249-252.

Cote, G.L. (2002) Subcutaneous fluorescence glucose sensing using a competitive binding assay. Abstr Pap Am Chem S 224, U116-U116.

Côté, G.L. and Robyt, J.F. (1982) Isolation and partial characterization of an extracellular glucansucrase from Leuconostoc mesenteroides NRRL B-1355 that synthesizes an alternating $(1 \rightarrow 6),(1 \rightarrow 3)$-a-D-glucan. Carbohyd Res 101, 57-74.

Craig, J., Lloyd, J.R., Tomlinson, K., Barber, L., Edwards, A., Wang, T.L., Martin, C., Hedley, C.L. and Smith, A.M. (1998) Mutations in the gene encoding starch synthase II profoundly alter amylopectin structure in pea embryos. The Plant cell 10, 413-426. 
Delatte, T., Umhang, M., Trevisan, M., Eicke, S., Thorneycroft, D., Smith, S.M. and Zeeman, S.C. (2006) Evidence for distinct mechanisms of starch granule breakdown in plants. Journal of Biological Chemistry 281, 1205012059.

Delgado-Escueta, A.V., Medina, M.T., Serratosa, J.M., Castroviejo, I.P., Gee, M.N., Weissbecker, K., Westling, B.W., Fong, C.Y., Alonso, M.E., Cordova, S., Shah, P., Khan, S., Sainz, J., Rubio-Donnadieu, F. and Sparkes, R.S. (1999) Mapping and positional cloning of common idiopathic generalized epilepsies: juvenile myoclonus epilepsy and childhood absence epilepsy. Advances in neurology 79, 351-374.

Delrue, B., Fontaine, T., Routier, F., Decq, A., Wieruszeski, J.M., Vandenkoornhuyse, N., Maddelein, M.L., Fournet, B. and Ball, S. (1992) Waxy Chlamydomonas-Reinhardtii - Monocellular Algal Mutants Defective in Amylose Biosynthesis and Granule-Bound Starch Synthase Activity Accumulate a Structurally Modified Amylopectin. J Bacteriol 174, 3612-3620.

Denyer, K., Foster, J. and Smith, A.M. (1995) The Contributions of Adenosine 5'-Diphosphoglucose Pyrophosphorylase and Starch-Branching Enzyme to the Control of Starch Synthesis in Developing Pea Embryos. Planta 197, 57-62.

Dhital, S., Shrestha, A.K. and Gidley, M.J. (2010) Relationship between granule size and in vitro digestibility of maize and potato starches. Carbohyd Polym 82, 480-488.

Dobruchowska, J.M., Gerwig, G.J., Kralj, S., Grijpstra, P., Leemhuis, H., Dijkhuizen, L. and Kamerling, J.P. (2012) Structural characterization of linear isomalto-/malto-oligomer products synthesized by the novel GTFB 4,6-alpha-glucanotransferase enzyme from Lactobacillus reuteri 121. Glycobiology 22, 517-528.

Doehlert, D.C. and Knutson, C.A. (1991) 2 Classes of Starch Debranching Enzymes from Developing Maize Kernels. Journal of plant physiology 138, 566-572.

Donath, H., Avtomonov, E.V., Sarraje, I., von Dahlen, K.H., El-Essawi, M., Lorberth, J. and Seo, B.S. (1998) Organoplatinum compounds VII - Trimethylplatinum fluoride [(CH3)(3)PtF](4), the missing link in organoplatinum cluster chemistry: its synthesis, crystal structure and a comparison to the crystal structure of [(CH3)(3)PtOH](4). J Organomet Chem 559, 191-196.

Edner, C., Li, J., Albrecht, T., Mahlow, S., Hejazi, M., Hussain, H., Kaplan, F., Guy, C., Smith, S.M., Steup, M. and Ritte, G. (2007) Glucan, water dikinase activity stimulates breakdown of starch granules by plastidial betaamylases. Plant physiology 145, 17-28.

Edwards, A., Fulton, D.C., Hylton, C.M., Jobling, S.A., Gidley, M., Rossner, U., Martin, C. and Smith, A.M. (1999) A combined reduction in activity of starch synthases II and III of potato has novel effects on the starch of tubers. Plant Journal 17, 251-261.

Edwards, A., Marshall, J., Sidebottom, C., Visser, R.G.F., Smith, A.M. and Martin, C. (1995) Biochemical and Molecular Characterization of a Novel Starch Synthase from Potato-Tubers. Plant Journal 8, 283-294.

Ellis, R.P., Cochrane, M.P., Dale, M.F.B., Duffus, C.M., Lynn, A., Morrison, I.M., Prentice, R.D.M., Swanston, J.S. and Tiller, S.A. (1998) Starch production and industrial use. Journal of the Science of Food and Agriculture 77, 289-311.

Emanuelle, S., Brewer, M.K., Meekins, D.A. and Gentry, M.S. (2016) Unique carbohydrate binding platforms employed by the glucan phosphatases. Cellular and molecular life sciences: CMLS.

Emes, M.J. and Neuhaus, H.E. (1997) Metabolism and transport in non-photosynthetic plastids. Journal of experimental botany 48, 1995-2005.

Espada, J. (1962) Enzymic Synthesis of Adenosine Diphosphate Glucose from Glucose 1-Phosphate and Adenosine Triphosphate. Journal of Biological Chemistry 237, 3577-\&.

Ferreira, S.J. (2011) Transcriptome based analysis of starch metabolism in Solanum tuberosum.

Ferretti, J.J., Gilpin, M.L. and Russell, R.R.B. (1987) Nucleotide-Sequence of a Glucosyltransferase Gene from Streptococcus-Sobrinus Mfe28. J Bacteriol 169, 4271-4278.

Fincher, G.B. (1989) Molecular and Cellular Biology Associated with Endosperm Mobilization in Germinating Cereal-Grains. Annu Rev Plant Phys 40, 305-346.

Firouzabadi, F.N., Kok-Jacon, G.A., Vincken, J.P., Ji, Q., Suurs, L.C.J.M. and Visser, R.G.F. (2007) Fusion proteins comprising the catalytic domain of mutansucrase and a starch-binding domain can alter the morphology of amylose-free potato starch granules during biosynthesis. Transgenic research 16, 645-656. 
Flipse, E., Huisman, J.G., Devries, B.J., Bergervoet, J.E.M., Jacobsen, E. and Visser, R.G.F. (1994) Expression of a Wild-Type Gbss Gene Introduced into an Amylose Free Potato Mutant by Agrobacterium-Tumefaciens and the Inheritance of the Inserts at the Microsporic Level. Theoretical and Applied Genetics 88, 369-375.

Flipse, E., Schippers, M.G., Janssen, E.M., Jacobsen, E. and Visser, R.G. (1996a) Expression of wild-type GBSS transgenes in the off-spring of partially and fully complementedamylose-free transformants of potato. Molecular Breeding 2, 211-218.

Flipse, E., StraatmanEngelen, I., Kuipers, A.G.J., Jacobsen, E. and Visser, R.G.F. (1996b) GBSS T-DNA inserts giving partial complementation of the amylose-free potato mutant can also cause co-suppression of the endogenous GBSS gene in a wild-type background. Plant molecular biology 31, 731-739.

Fredriksson, H., Silverio, J., Andersson, R., Eliasson, A.C. and Aman, P. (1998) The influence of amylose and amylopectin characteristics on gelatinization and retrogradation properties of different starches. Carbohyd Polym 35, 119-134.

Frohberg, C. (2008) Genetically modified plants which synthesize a starch having increased swelling power. Int Patent WO 08/017518.

Frohberg, C., Koetting, O., Ritte, G. and Steup, M. (2011) Plants with increased activity of multiple starch phosphorylating enzymes. Patent CA2558747A1.

Frohberg, C., Koetting, O., Ritte, G. and Steup, M. (2013) Plants with increased activity of a starch phosphorylating enzyme. Patent US20110027447.

Fulton, D.C., Edwards, A., Pilling, E., Robinson, H.L., Fahy, B., Seale, R., Kato, L., Donald, A.M., Geigenberger, P., Martin, C. and Smith, A.M. (2002) Role of granule-bound starch synthase in determination of amylopectin structure and starch granule morphology in potato. Journal of Biological Chemistry 277, 10834-10841.

Geigenberger, P., Stitt, M. and Fernie, A. (2004) Metabolic control analysis and regulation of the conversion of sucrose to starch in growing potato tubers. Plant, cell \& environment 27, 655-673.

Gentry, M.S., Dowen, R.H., 3rd, Worby, C.A., Mattoo, S., Ecker, J.R. and Dixon, J.E. (2007) The phosphatase laforin crosses evolutionary boundaries and links carbohydrate metabolism to neuronal disease. The Journal of cell biology 178, 477-488.

Glaring, M.A., Zygadlo, A., Thorneycroft, D., Schulz, A., Smith, S.M., Blennow, A. and Baunsgaard, L. (2007) An extra-plastidial alpha-glucan, water dikinase from Arabidopsis phosphorylates amylopectin in vitro and is not necessary for transient starch degradation. Journal of experimental botany 58, 3949-3960.

Grimaud, F., Rogniaux, H., James, M.G., Myers, A.M. and Planchot, V. (2008) Proteome and phosphoproteome analysis of starch granule-associated proteins from normal maize and mutants affected in starch biosynthesis. Journal of experimental botany 59, 3395-3406.

Grommers, H. and Van Der Krogt, D. (2009) Potato starch: production, modifications and uses. Starch: Chemistry and Technology 3.

Haghayegh, G. and Schoenlechner, R. (2011) Physically modified starches: A review. J Food Agric Environ 9, $27-29$.

Hamada, S. and Slade, H.D. (1980) Biology, Immunology, and Cariogenicity of Streptococcus-Mutans. Microbiol Rev 44, 331-384.

Hammer, Ø., Harper, D. and Ryan, P. (2001) Past: Paleontological Statistics Software Package for education and data analysis. Paleontología Electrónica 4: 1-9. URL:< http://palaeo-electronica. org/2001_1/past/ issue1_01.html.

Hansen, P.I., Larsen, F.H., Motawia, S.M., Blennow, A., Spraul, M., Dvortsak, P. and Engelsen, S.B. (2008) Structure and hydration of the amylopectin trisaccharide building blocks--Synthesis, NMR, and molecular dynamics. Biopolymers 89, 1179-1193.

Heldt, H.W. and Pfaff, E. (1969) Adenine Nucleotide Translocation in Mitochondria - Quantitative Evaluation of Correlation between Phosphorylation of Endogenous and Exogenous Adp in Mitochondria. Eur J Biochem 10, 494-\&.

Hizukuri, S., Tabata, S. and Nikuni, Z. (1970) Studies on Starch Phosphate .1. Estimation of Glucose-6-Phosphate Residues in Starch and Presence of Other Bound Phosphate(S). Starke 22, 338.

Hizukuri, S. and Takeda, Y. (1970) Activation of the D to I form conversion of glycogen synthetase by glucose 6-phosphate in bovine spleen. Biochimica et biophysica acta 212, 179-181. 
Hofvander, P., Andersson, M., Larsson, C.T. and Larsson, H. (2004) Field performance and starch characteristics of high-amylose potatoes obtained by antisense gene targeting of two branching enzymes. Plant Biotechnology Journal 2, 311-320.

Hoover, R. (2001) Composition, molecular structure, and physicochemical properties of tuber and root starches: a review. Carbohyd Polym 45, 253-267.

Hovenkamp-Hermelink, J.H.M., De Vries, J.N., Adamse, P., Jacobsen, E., Witholt, B. and Feenstra, W.J. (1988) Rapid estimation of the amylose/amylopectin ratio in small amounts of tuber and leaf tissue of the potato. Potato Research 31, 241-246.

Hovenkamphermelink, J.H.M., Jacobsen, E., Ponstein, A.S., Visser, R.G.F., Vosscheperkeuter, G.H., Bijmolt, E.W., Devries, J.N., Witholt, B. and Feenstra, W.J. (1987) Isolation of an Amylose-Free Starch Mutant of the Potato (Solanum-Tuberosum-L). Theoretical and Applied Genetics 75, 217-221.

Hsieh, J. (1988) GENETIC-STUDIES ON THE WX-GENE OF SORGHUM [SORGHUM-BICOLOR (L) MOENCH]. 1. EXAMINATION OF THE PROTEIN PRODUCT OF THE WAXY LOCUS. Botanical Bulletin of Academia Sinica 29, 293-299.

Huang, X.-F., Nazarian-Firouzabadi, F., Vincken, J.-P., Ji, Q., Suurs, L.C.J.M., Visser, R.G.F. and Trindade, L.M. (2013) Expression of an engineered granule-bound Escherichia coli glycogen branching enzyme in potato results in severe morphological changes in starch granules. Plant Biotechnology Journal 11, 470-479.

Huang, X.-F., Nazarian-Firouzabadi, F., Vincken, J.-P., Ji, Q., Visser, R.G. and Trindade, L.M. (2014) Expression of an amylosucrase gene in potato results in larger starch granules with novel properties. Planta 240, 409421.

Ihemere, U., Arias-Garzon, D., Lawrence, S. and Sayre, R. (2006) Genetic modification of cassava for enhanced starch production. Plant Biotechnology Journal 4, 453-465.

Imberty, A., Chanzy, H., Pérez, S., Bulèon, A. and Tran, V. (1988) The double-helical nature of the crystalline part of A-starch. Journal of molecular biology 201, 365-378.

Imberty, A. and Perez, S. (1988) A revisit to the three-dimensional structure of B-type starch. Biopolymers 27, 1205-1221.

Isherwoo, F.A. (1973) Starch-Sugar Interconversion in Solanum-Tuberosum. Phytochemistry 12, 2579-2591.

James, M.G., Robertson, D.S. and Myers, A.M. (1995) Characterization of the Maize Gene Sugary1, a Determinant of Starch Composition in Kernels. The Plant cell 7, 417-429.

Jane, J., Chen, Y.Y., Lee, L.F., McPherson, A.E., Wong, K.S., Radosavljevic, M. and Kasemsuwan, T. (1999) Effects of amylopectin branch chain length and amylose content on the gelatinization and pasting properties of starch. Cereal Chem 76, 629-637.

Janecek, S., Svensson, B. and MacGregor, E.A. (2003) Relation between domain evolution, specificity, and taxonomy of the alpha-amylase family members containing a C-terminal starch-binding domain. Eur J Biochem 270, 635-645.

Jeanes, A., Haynes, W.C., Wilham, C.A., Rankin, J.C., Melvin, E.H., Austin, M.J., Cluskey, J.E., Fisher, B.E., Tsuchiya, H.M. and Rist, C.E. (1954) Characterization and Classification of Dextrans from 96 Strains of Bacteria. J Am Chem Soc 76, 5041-5052.

Jenkins, J.P.J., Cameron, R.E. and Donald, A.M. (1993a) A Universal Feature in the Structure of Starch Granules from Different Botanical Sources. Starch-Starke 45, 417-420.

Jenkins, P.J., Cameron, R.E. and Donald, A.M. (1993b) A universal feature in the structure of starch granules from different botanical sources. Starch-Stärke 45, 417-420.

Ji, Q., Oomen, R.J., Vincken, J.P., Bolam, D.N., Gilbert, H.J., Suurs, L.C. and Visser, R.G. (2004) Reduction of starch granule size by expression of an engineered tandem starch-binding domain in potato plants. Plant Biotechnol J 2, 251-260.

Ji, Q., Vincken, J.P., Suurs, L.C. and Visser, R.G. (2003) Microbial starch-binding domains as a tool for targeting proteins to granules during starch biosynthesis. Plant molecular biology 51, 789-801.

Jobling, S. (2004) Improving starch for food and industrial applications. Current opinion in plant biology 7, 210218. 
Jobling, S.A., Schwall, G.P., Westcott, R.J., Sidebottom, C.M., Debet, M., Gidley, M.J., Jeffcoat, R. and Safford, R. (1999) A minor form of starch branching enzyme in potato (Solanum tuberosum L.) tubers has a major effect on starch structure: cloning and characterisation of multiple forms of SBE A. Plant Journal 18, 163-171.

Jobling, S.A., Westcott, R.J., Tayal, A., Jeffcoat, R. and Schwall, G.P. (2002) Production of a freeze-thaw-stable potato starch by antisense inhibition of three starch synthase genes. Nature biotechnology 20, 295-299.

Kaplan, F. and Guy, C.L. (2005) RNA interference of Arabidopsis beta-amylase8 prevents maltose accumulation upon cold shock and increases sensitivity of PSII photochemical efficiency to freezing stress. Plant Journal 44, 730-743.

Karim, A., Toon, L., Lee, V., Ong, W., Fazilah, A. and Noda, T. (2007) Effects of phosphorus contents on the gelatinization and retrogradation of potato starch. Journal of food science 72, C132-C138.

Katayama, K., Komae, K., Kohyama, K., Kato, T., Tamiya, S. and Komaki, K. (2002) New Sweet Potato Line having low Gelatinization Temperature and altered Starch Structure. Starch-Starke 54, 51-57.

Kaur, B., Ariffin, F., Bhat, R. and Karim, A.A. (2012) Progress in starch modification in the last decade. Food Hydrocolloid 26, 398-404.

Kaur, L., Singh, J., McCarthy, O.J. and Singh, H. (2007) Physico-chemical, rheological and structural properties of fractionated potato starches. J Food Eng 82, 383-394.

Kim, H.S. and Huber, K.C. (2008) Channels within soft wheat starch A- and B-type granules. Journal of Cereal Science 48, 159-172.

Kim, W., Johnson, J.W., Graybosch, R.A. and Gaines, C.S. (2003) Physicochemical properties and end-use quality of wheat starch as a function of waxy protein alleles. Journal of Cereal Science 37, 195-204.

Kimura, T., Otani, M., Noda, T., Ideta, O., Shimada, T. and Saito, A. (2001) Absence of amylose in sweet potato [Ipomoea batatas (L.) Lam.] following the introduction of granule-bound starch synthase I cDNA. Plant cell reports 20, 663-666.

Kok-Jacon, G.A., Ji, Q., Vincken, J.P. and Visser, R.G. (2003) Towards a more versatile alpha-glucan biosynthesis in plants. Journal of plant physiology 160, 765-777.

Kok-Jacon, G.A., Vincken, J.P., Suurs, L.C. and Visser, R.G. (2005a) Mutan produced in potato amyloplasts adheres to starch granules. Plant Biotechnol J 3, 341-351.

Kok-Jacon, G.A., Vincken, J.P., Suurs, L.C., Wang, D., Liu, S. and Visser, R.G. (2007) Expression of alternansucrase in potato plants. Biotechnology letters 29, 1135-1142.

Kok-Jacon, G.A., Vincken, J.P., Suurs, L.C.J.M. and Visser, R.G.F. (2005b) Mutan produced in potato amyloplasts adheres to starch granules. Plant Biotechnology Journal 3, 341-351.

Kok-Jacon, G.A., Vincken, J.P., Suurs, L.C.J.M., Wang, D.N., Liu, S.Y. and Visser, R.G.F. (2005c) Production of dextran in transgenic potato plants. Transgenic research 14, 385-395.

Kooistra, E. (1962) On the differences between smooth and three types of wrinkled peas. Euphytica 11, $357-373$.

Kortstee, A.J. (1997) Modification of potato starch composition by introduction and expression of bacterial branching enzyme genes. [S.I.]: Kortstee.

Kortstee, A.J., Vermeesch, A.M., de Vries, B.J., Jacobsen, E. and Visser, R.G. (1996) Expression of Escherichia coli branching enzyme in tubers of amylose-free transgenic potato leads to an increased branching degree of the amylopectin. The Plant journal : for cell and molecular biology 10, 83-90.

Korus, J., Tomasik, P. and Lii, C. (2003) Microcapsules from starch granules. J Microencapsul 20, 47-56.

Kossmann, J., Buttcher, V., Abel, G.J.W., Duwenig, E., Emmermann, M., Frohberg, C., Lloyd, J.R., Lorberth, R., Springer, F., Welsh, T. and Willmitzer, L. (1997) Starch biosynthesis and modification of starch structure in transgenic plants. Macromol Symp 120, 29-38.

Kossmann, J. and Lloyd, J. (2000) Understanding and influencing starch biochemistry. Critical reviews in biochemistry and molecular biology 35, 141-196.

Kotting, O., Pusch, K., Tiessen, A., Geigenberger, P., Steup, M. and Ritte, G. (2005) Identification of a novel enzyme required for starch metabolism in Arabidopsis leaves. The phosphoglucan, water dikinase. Plant physiology 137, 242-252. 
Kotting, O., Santelia, D., Edner, C., Eicke, S., Marthaler, T., Gentry, M.S., Comparot-Moss, S., Chen, J., Smith, A.M., Steup, M., Ritte, G. and Zeeman, S.C. (2009) STARCH-EXCESS4 is a laforin-like Phosphoglucan phosphatase required for starch degradation in Arabidopsis thaliana. The Plant cell 21, 334-346.

Kozlov, S.S., Blennow, A., Krivandin, A.V. and Yuryev, V.P. (2007) Structural and thermodynamic properties of starches extracted from GBSS and GWD suppressed potato lines. International journal of biological macromolecules 40, 449-460.

Kralj, S., van Geel-Schutten, G.H., Dondorff, M.M.G., Kirsanovs, S., van der Maarel, M.J.E.C. and Dijkhuizen, L. (2004) Glucan synthesis in the genus Lactobacillus: isolation and characterization of glucansucrase genes, enzymes and glucan products from six different strains. Microbiol-Sgm 150, 3681-3690.

Krohn, B., Stark, D., Barry, G., Preiss, J. and Kishore, G. (1994) Modification of starch structure in transgenic potato. Plant Physiol [Suppl] 105, 37.

Kruger, N.J. (1990) Carbohydrate synthesis and degradation. Plant physiology, biochemistry and molecular biology 1, 59-76.

Krylov, D.M., Wolf, Y.I., Rogozin, I.B. and Koonin, E.V. (2003) Gene loss, protein sequence divergence, gene dispensability, expression level, and interactivity are correlated in eukaryotic evolution. Genome research 13, 2229-2235.

Kuipers, A., Jacobsen, E. and Visser, R. (1994) Formation and Deposition of Amylose in the Potato Tuber Starch Granule Are Affected by the Reduction of Granule-Bound Starch Synthase Gene Expression. The Plant cell 6, 43-52.

Laemmli, U.K. (1970) Cleavage of structural proteins during the assembly of the head of bacteriophage T4. Nature 227, 680-685.

Lafora, G. and Glueck, B. (1911) Contribution to the histopathology and pathogenesis of myoclonic epilepsy. Bull Gov Hosp Insane 3, 111.

Lanahan, M. and Basu, S. (2004) Modified starch, uses, methods for production thereof. Google Patents.

LanahanMichael, B. and Ship, S.B. (2005) Modified starch, uses, methods for production thereof.

Leathers, T.D., Nunnally, M.S., Ahlgren, J.A. and Cote, G.L. (2003) Characterization of a novel modified alternan. Carbohyd Polym 54, 107-113.

Leathers, T.D., Nunnally, M.S. and Cote, G.L. (2002) Modification of alternan by novel Penicillium spp. Journal of industrial microbiology \& biotechnology 29, 177-180.

Leemhuis, H., Dobruchowska, J.M., Ebbelaar, M., Faber, F., Buwalda, P.L., van der Maarel, M.J.E.C., Kamerling, J.P. and Dijkhuizen, L. (2014) Isomalto/Malto-Polysaccharide, A Novel Soluble Dietary Fiber Made Via Enzymatic Conversion of Starch. Journal of agricultural and food chemistry 62, 12034-12044.

Lewis, C.E., Lancaster, J.E., Meredith, P. and Walker, J.R.L. (1994) Starch Metabolism during Growth and Storage of Tubers of 2 New-Zealand Potato Cultivars. New Zeal J Crop Hort 22, 295-304.

Li, Y.T., Han, D.X., Hu, G.R., Dauvillee, D., Sommerfeld, M., Ball, S. and Hua, Q. (2010) Chlamydomonas starchless mutant defective in ADP-glucose pyrophosphorylase hyper-accumulates triacylglycerol. Metab Eng 12, 387-391.

Lii, C.Y., Tomasik, P., Hung, W.L., Yen, M.T. and Lai, V.M.F. (2003) Granular starches as dietary fibre and natural microcapsules. Int J Food Sci Tech 38, 677-685.

Lindeboom, N., Chang, P.R. and Tyler, R.T. (2004) Analytical, biochemical and physicochemical aspects of starch granule size, with emphasis on small granule starches: A review. Starch-Starke 56, 89-99.

Liu, Y., Wang, Y., Wu, C., Liu, Y. and Zheng, P. (2006) Dimerization of Laforin is required for its optimal phosphatase activity, regulation of GSK3 beta phosphorylation, and Wnt signaling. Journal of Biological Chemistry 281, 34768-34774.

Livak, K.J. and Schmittgen, T.D. (2001) Analysis of relative gene expression data using real-time quantitative PCR and the 2(-Delta Delta C(T)) Method. Methods 25, 402-408.

Lloyd, J., Landschutze, V. and Kossmann, J. (1999) Simultaneous antisense inhibition of two starch-synthase isoforms in potato tubers leads to accumulation of grossly modified amylopectin. Biochem. J 338, 515521. 
Lloyd, J.R. and Kossmann, J. (2015) Transitory and storage starch metabolism: two sides of the same coin? Current opinion in biotechnology 32, 143-148.

Lorberth, R., Ritte, G., Willmitzer, L. and Kossmann, J. (1998) Inhibition of a starch-granule-bound protein leads to modified starch and repression of cold sweetening. Nature biotechnology 16, 473-477.

Lusser, M., Parisi, C., Plan, D. and Rodriguez-Cerezo, E. (2012) Deployment of new biotechnologies in plant breeding. Nature biotechnology 30, 231-239.

Machovic, M., Svensson, B., Ann MacGregor, E. and Janecek, S. (2005) A new clan of CBM families based on bioinformatics of starch-binding domains from families CBM20 and CBM21. Febs Journal 272, 54975513.

Mahlow, S., Hejazi, M., Kuhnert, F., Garz, A., Brust, H., Baumann, O. and Fettke, J. (2014) Phosphorylation of transitory starch by alpha-glucan, water dikinase during starch turnover affects the surface properties and morphology of starch granules. The New phytologist 203, 495-507.

Manners, D.J. (1989) Recent developments in our understanding of amylopectin structure. Carbohyd Polym 11, $87-112$.

Marshall, J., Sidebottom, C., Debet, M., Martin, C., Smith, A.M. and Edwards, A. (1996) Identification of the major starch synthase in the soluble fraction of potato tubers. The Plant cell 8, 1121-1135.

Mason, W. (2009) Starch use in foods. Starch: Chemistry and technology, 745-795.

Matheson, N.K. (1996) The chemical structure of amylose and amylopectin fractions of starch from tobacco leaves during development and diurnally nocturnally. Carbohyd Res 282, 247-262.

Mikkelsen, R., Baunsgaard, L. and Blennow, A. (2004) Functional characterization of alpha-glucan,water dikinase, the starch phosphorylating enzyme. The Biochemical journal 377, 525-532.

Minassian, B.A., Sainz, J., Serratosa, J.M., Gee, M., Sakamoto, L.M., Bohlega, S., Geoffroy, G., Barr, C., Scherer, S.W., Tomiyasu, U., Carpenter, S., Wigg, K., Sanghvi, A.V. and Delgado-Escueta, A.V. (1999) Genetic locus heterogeneity in Lafora's progressive myoclonus epilepsy. Ann Neurol 45, 262-265.

Moire, L., Rezzonico, E. and Poirier, Y. (2003) Synthesis of novel biomaterials in plants. Journal of plant physiology $160,831-839$.

Monchois, V., Vignon, M. and Russell, R.R.B. (1999) Isolation of key amino acid residues at the N-terminal end of the core region Streptococcus downei glucansucrase, GTF-I. Applied microbiology and biotechnology 52, 660-665.

Monchois, V., Willemot, R.M., RemaudSimeon, M., Croux, C. and Monsan, P. (1996) Cloning and sequencing of a gene coding for a novel dextransucrase from Leuconostoc mesenteroides NRRL B-1299 synthesizing only alpha(1-6) and alpha(1-3) linkages. Gene 182, 23-32.

Mooser, G. (1992) 5 Glycosidases and Glycosyltransferases. The enzymes 20, 187-233.

Morrison, I.M., Cochrane, M.P., Cooper, A.M., Dale, M.F.B., Duffus, C.M., Ellis, R.P., Lynn, A., Mackay, G.R., Paterson, L.J. and Prentice, R.D.M. (2001) Potato starches: variation in composition and properties between three genotypes grown at two different sites and in two different years. Journal of the Science of Food and Agriculture 81, 319-328.

Morrison, W. (1964) A fast, simple and reliable method for the microdetermination of phosphorus in biological materials. Analytical biochemistry 7, 218-224.

Mouille, G., Maddelein, M.L., Libessart, N., Talaga, P., Decq, A., Delrue, B. and Ball, S. (1996) Preamylopectin processing: A mandatory step for starch biosynthesis in plants. The Plant cell 8, 1353-1366.

Muller-Rober, B.T., Kossmann, J., Hannah, L.C., Willmitzer, L. and Sonnewald, U. (1990) One of two different ADP-glucose pyrophosphorylase genes from potato responds strongly to elevated levels of sucrose. Molecular \& general genetics: MGG 224, 136-146.

Myers, A.M., Morell, M.K., James, M.G. and Ball, S.G. (2000) Recent progress toward understanding biosynthesis of the amylopectin crystal. Plant Physiology 122, 989-998.

Naguleswaran, S., Li, J.H., Vasanthan, T. and Bressler, D. (2011) Distribution of Granule Channels, Protein, and Phospholipid in Triticale and Corn Starches as Revealed by Confocal Laser Scanning Microscopy. Cereal Chem 88, 87-94.

Nakamura, T., Yamamori, M., Hirano, H., Hidaka, S. and Nagamine, T. (1995) Production of Waxy (Amylose-Free) Wheats. Molecular \& General Genetics 248, 253-259. 
Nakamura, Y. (1996) Some properties of starch debranching enzymes and their possible role in amylopectin biosynthesis. Plant Sci 121, 1-18.

Napoli, C., Lemieux, C. and Jorgensen, R. (1990) Introduction of a Chimeric Chalcone Synthase Gene into Petunia Results in Reversible Co-Suppression of Homologous Genes in trans. The Plant cell 2, 279-289.

Nazarian-Firouzabadi, F., Trindade, L.M. and Visser, R.G.F. (2012) Production of small starch granules by expression of a tandem-repeat of a family 20 starch-binding domain (SBD3-SBD5) in an amylose-free potato genetic background. Funct Plant Biol 39, 146-155.

Nazarian Firouzabadi, F., Vincken, J.P., Ji, Q., Suurs, L.C. and Visser, R.G. (2007) Expression of an engineered granule-bound Escherichia coli maltose acetyltransferase in wild-type and amf potato plants. Plant Biotechnol J 5, 134-145.

Nelson, O.E. and Rines, H.W. (1962) Enzymtic Deficiency in Waxy Mutant of Maize. Biochem Bioph Res Co 9, 297300.

Neuhaus, H.E. and Emes, M.J. (2000) Nonphotosynthetic metabolism in plastids. Annu Rev Plant Phys 51, $111-140$.

Nicot, N., Hausman, J.F., Hoffmann, L. and Evers, D. (2005) Housekeeping gene selection for real-time RT-PCR normalization in potato during biotic and abiotic stress. Journal of experimental botany 56, 2907-2914.

Nierle, W. (1990) The influence of the manufacturing conditions on the properties of phosphated corn starch and their applications.

Noda, T., Kimura, T., Otani, M., Ideta, O., Shimada, T., Saito, A. and Suda, I. (2002) Physicochemical properties of amylose-free starch from transgenic sweet potato. Carbohyd Polym 49, 253-260.

Noda, T., Takigawa, S., Matsuura-Endo, C., Suzuki, T., Hashimoto, N., Kottearachchi, N., Yamauchi, H. and Zaidul, I. (2008) Factors affecting the digestibility of raw and gelatinized potato starches. Food Chemistry 110, 465-470.

Noda, T., Tsuda, S., Mori, M., Takigawa, S., Matsuura-Endo, C., Saito, K., Mangalika, W.H.A., Hanaoka, A., Suzuki, Y. and Yamauchi, H. (2004) The effect of harvest dates on the starch properties of various potato cultivars. Food Chemistry 86, 119-125.

Ohdan, K., Kuriki, T., Takata, H., Kaneko, H. and Okada, S. (2000) Introduction of raw starch-binding domains into Bacillus subtilis alpha-amylase by fusion with the starch-binding domain of Bacillus cyclomaltodextrin glucanotransferase. Appl Environ Microb 66, 3058-3064.

Okuno, K. and Sakaguchi, S. (1982) Inheritance of Starch Characteristics in Perisperm of AmaranthusHypochondriacus. J Hered 73, 467-467.

Orzechowski, S., Grabowska, A., Sitnicka, D., Siminska, J., Felus, M., Dudkiewicz, M., Fudali, S. and Sobczak, M. (2013) Analysis of the expression, subcellular and tissue localisation of phosphoglucan, water dikinase (PWD/GWD3) in Solanum tuberosum L.: a bioinformatics approach for the comparative analysis of two a-glucan, water dikinases (GWDs) from Solanum tuberosum L. Acta Physiologiae Plantarum 35, 483-500.

Palevitz, B.A. and Newcomb, E.H. (1970) A study of sieve element starch using sequential enzymatic digestion and electron microscopy. The Journal of cell biology 45, 383-398.

Pan, D. and Nelson, O.E. (1984) A Debranching Enzyme Deficiency in Endosperms of the Sugary-1 Mutants of Maize. Plant physiology 74, 324-328.

Parada, J. and Aguilera, J.M. (2009) In vitro Digestibility and Glycemic Response of Potato Starch is Related to Granule Size and Degree of Gelatinization. Journal of Food Science 74, E34-E38.

Pfister, B. and Zeeman, S.C. (2016) Formation of starch in plant cells. Cellular and molecular life sciences: CMLS.

Pilling, E. and Smith, A.M. (2003) Growth ring formation in the starch granules of potato tubers. Plant physiology 132, 365-371.

Preiss, J., Kumar, A. and Ghosh, P. (1988) A Study of the Catalytic and Regulatory Functions of a Sugar Nucleotide Synthetase Using Invitro and Invivo Mutagenesis Techniques. Glycoconjugate journal 5, 365-365.

Preiss, J. and Sivak, M.N. (1998) Biochemistry, molecular biology and regulation of starch synthesis. Genetic engineering 20, 177-223.

Radchuk, V.V., Borisjuk, L., Sreenivasulu, N., Merx, K., Mock, H.P., Rolletschek, H., Wobus, U. and Weschke, W. (2009) Spatiotemporal Profiling of Starch Biosynthesis and Degradation in the Developing Barley Grain. Plant physiology 150, 190-204. 
Ral, J.P., Bowerman, A.F., Li, Z., Sirault, X., Furbank, R., Pritchard, J.R., Bloemsma, M., Cavanagh, C.R., Howitt, C.A. and Morell, M.K. (2012) Down-regulation of Glucan, Water-Dikinase activity in wheat endosperm increases vegetative biomass and yield. Plant Biotechnol $J$ 10, 871-882.

Regina, A., Bird, A., Topping, D., Bowden, S., Freeman, J., Barsby, T., Kosar-Hashemi, B., Li, Z.Y., Rahman, S. and Morell, M. (2006) High-amylose wheat generated by RNA interference improves indices of large-bowel health in rats. Proceedings of the National Academy of Sciences of the United States of America 103, 35463551.

Ridout, M.J., Gunning, A.P., Parker, M.L., Wilson, R.H. and Morris, V.J. (2002) Using AFM to image the internal structure of starch granules. Carbohyd Polym 50, 123-132.

Ritchie, S., Swanson, S.J. and Gilroy, S. (2000) Physiology of the aleurone layer and starchy endosperm during grain development and early seedling growth: new insights from cell and molecular biology. Seed Sci Res 10, 193-212.

Ritte, G., Eckermann, N., Haebel, S., Lorberth, R. and Steup, M. (2000) Compartmentation of the Starch-Related R1 Protein in Higher Plants. Starch-Stärke 52, 145-149.

Ritte, G., Edner, C., Li, J. and Smith, S. (2007) Glucan, water dikinase activity stimulates breakdown of granular starch by chloroplastic beta-amylases. Photosynth Res 91, 237-237.

Ritte, G., Heydenreich, M., Mahlow, S., Haebel, S., Kotting, O. and Steup, M. (2006) Phosphorylation of C6- and C3-positions of glucosyl residues in starch is catalysed by distinct dikinases. FEBS letters 580, 4872-4876.

Ritte, G., Lloyd, J.R., Eckermann, N., Rottmann, A., Kossmann, J. and Steup, M. (2002) The starch-related R1 protein is an alpha -glucan, water dikinase. Proceedings of the National Academy of Sciences of the United States of America 99, 7166-7171.

Robyt, J. (1995a) Properties and uses of dextrans and related glucans. In: Abstr Pap Am Chem S pp. 86-PMSE. AMER CHEMICAL SOC PO BOX 57136, WASHINGTON, DC 20037-0136.

Robyt, J.F. (1995b) Mechanisms in the glucansucrase synthesis of polysaccharides and oligosaccharides from sucrose. Advances in carbohydrate chemistry and biochemistry 51, 133-168.

Roper, H. (2002) Renewable raw materials in Europe - Industrial utilisation of starch and sugar. Starch-Starke 54, 89-99.

Rzhetsky, A. and Nei, M. (1992) A simple method for estimating and testing minimum-evolution trees. Mol. Biol. Evol 9, 945-967.

Safford, R., Jobling, S.A., Sidebottom, C.M., Westcott, R.J., Cooke, D., Tober, K.J., Strongitharm, B.H., Russell, A.L. and Gidley, M.J. (1998a) Consequences of antisense RNA inhibition of starch branching enzyme activity on properties of potato starch. Carbohyd Polym 35, 155-168.

Safford, R., Jobling, S.A., Sidebottom, C.M., Westcott, R.J., Cooke, D., Tober, K.J., Strongitharm, B.H., Russell, A.L. and Gidley, M.J. (1998b) Consequences of antisense RNA inhibition of starch branching enzyme activity on properties of potato starch. Carbohyd Polym 35, 155-168.

Sakai, M., Austin, J., Witmer, F. and Trueb, L. (1970) Studies in myoclonus epilepsy (Lafora body form). II. Polyglucosans in the systemic deposits of myoclonus epilepsy and in corpora amylacea. Neurology 20, 160-176.

Santelia, D., Kotting, O., Seung, D., Schubert, M., Thalmann, M., Bischof, S., Meekins, D.A., Lutz, A., Patron, N., Gentry, M.S., Allain, F.H. and Zeeman, S.C. (2011) The phosphoglucan phosphatase like sex Four2 dephosphorylates starch at the C3-position in Arabidopsis. The Plant cell 23, 4096-4111.

Santelia, D. and Zeeman, S.C. (2011) Progress in Arabidopsis starch research and potential biotechnological applications. Current opinion in biotechnology 22, 271-280.

Satoh, H., Nishi, A., Yamashita, K., Takemoto, Y., Tanaka, Y., Hosaka, Y., Sakurai, A., Fujita, N. and Nakamura, Y. (2003) Starch-branching enzyme I-deficient mutation specifically affects the structure and properties of starch in rice endosperm. Plant physiology 133, 1111-1121.

Scheidig, A., Frohlich, A., Schulze, S., Lloyd, J.R. and Kossmann, J. (2002) Downregulation of a chloroplast-targeted beta-amylase leads to a starch-excess phenotype in leaves. Plant Journal 30, 581-591.

Schewe, G., Knies, P., Amati, S.F., Loerz, H., Becker, D., Uwer, U., Landschuetze, V., Pilling, J. and Krohberg, K. (2002) Monocotyledon plant cells and plants which synthesise modified starch. In: Int Patent WO 02/34923. 
Schunemann, D., Borchert, S., Flugge, U.I. and Heldt, H.W. (1993) Adp/Atp Translocator from Pea Root Plastids Comparison with Translocators from Spinach-Chloroplasts and Pea Leaf Mitochondria. Plant physiology 103, 131-137.

Schwall, G.P., Safford, R., Westcott, R.J., Jeffcoat, R., Tayal, A., Shi, Y.C., Gidley, M.J. and Jobling, S.A. (2000) Production of very-high-amylose potato starch by inhibition of SBE A and B. Nature biotechnology 18, 551-554.

Serratosa, J.M., Gomez-Garre, P., Gallardo, M.E., Anta, B., de Bernabe, D.B., Lindhout, D., Augustijn, P.B., Tassinari, C.A., Malafosse, R.M., Topcu, M., Grid, D., Dravet, C., Berkovic, S.F. and de Cordoba, S.R. (1999) A novel protein tyrosine phosphatase gene is mutated in progressive myoclonus epilepsy of the Lafora type (EPM2). Hum Mol Genet 8, 345-352.

Seymour, F.K., Knapp, R.D., Chen, E.C., Bishop, S.H. and Jeanes, A. (1979) Structural analysis of Leuconostoc dextrans containing 3-O-a-d-glucosylated a-d-glucosyl residues in both linear-chain and branch-point positions, or only in branch-point positions, by methylation and by $13 \mathrm{CN}$. MR spectroscopy. Carbohyd Res 74, 41-62.

Seymour, F.R. and Knapp, R.D. (1980) Unusual Dextrans .13. Structural-Analysis of Dextrans, from Strains of Leuconostoc and Related Genera, That Contain 3-O-Alpha-D-Glucosylated Alpha-D-Glucopyranosyl Residues at the Branch-Points, or in Consecutive, Linear Positions. Carbohyd Res 81, 105-129.

Seymour, F.R., Slodki, M.E., Plattner, R.D. and Jeanes, A. (1977) Six unusual dextrans: methylation structural analysis by combined glc - ms of per-O-acetyl-aldononitriles. Carbohyd Res 53, 153-166.

Sharma, R., Sissons, M.J., Rathjen, A.J. and Jenner, C.F. (2002) The null-4A allele at the wax locus in durum wheat affects pasta cooking quality. Journal of Cereal Science 35, 287-297.

Shewmaker, C.K., Boyer, C.D., Wiesenborn, D.P., Thompson, D.B., Boersig, M.R., Oakes, J.V. and Stalker, D.M. (1994) Expression of Escherichia-Coli Glycogen-Synthase in the Tubers of Transgenic Potatoes (SolanumTuberosum) Results in a Highly Branched Starch. Plant physiology 104, 1159-1166.

Shrestha, A.K. and Halley, P.J. (2014) Starch Modification to develop novel starch-biopolymer blends: state of art and perspectives.

Sidebotham, R.L. (1974) Dextrans. Advances in carbohydrate chemistry and biochemistry 30, 371-444.

Simpson, C.L., Cheetham, N.W.H., Giffard, P.M. and Jacques, N.A. (1995) 4 Glucosyltransferases, Gtfj, Gtfk, Gtfl and Gtfm, from Streptococcus-Salivarius Atcc-25975. Microbiol-Uk 141, 1451-1460.

Singh, J., Dartois, A. and Kaur, L. (2010) Starch digestibility in food matrix: a review. Trends in Food Science \& Technology 21, 168-180.

Singh, N., Sandhu, K.S. and Kaur, M. (2005) Physicochemical properties including granular morphology, amylose content, swelling and solubility, thermal and pasting properties of starches from normal, waxy, high amylose and sugary corn. Progress in food biopolymer research 1, 43-54.

Skeffington, A.W., Graf, A., Duxbury, Z., Gruissem, W. and Smith, A.M. (2014) Glucan, Water Dikinase Exerts Little Control over Starch Degradation in Arabidopsis Leaves at Night. Plant physiology 165, 866-879.

Slade, A.J., McGuire, C., Loeffler, D., Mullenberg, J., Skinner, W., Fazio, G., Holm, A., Brandt, K.M., Steine, M.N. and Goodstal, J.F. (2012) Development of high amylose wheat through TILLING. BMC plant biology $12,1$.

Smith, A.M., Bettey, M. and Bedford, I.D. (1989) Evidence That the Rb Locus Alters the Starch Content of Developing Pea Embryos through an Effect on Adp Glucose Pyrophosphorylase. Plant physiology 89, 1279-1284.

Smith, A.M., Denyer, K. and Martin, C. (1997) The synthesis of the starch granule. Annu Rev Plant Phys 48, 65-87.

Smith, S.M., Fulton, D.C., Chia, T., Thorneycroft, D., Chapple, A., Dunstan, H., Hylton, C., Zeeman, S.C. and Smith, A.M. (2004) Diurnal changes in the transcriptome encoding enzymes of starch metabolism provide evidence for both transcriptional and posttranscriptional regulation of starch metabolism in Arabidopsis leaves. Plant physiology 136, 2687-2699.

Snell, K.D., Singh, V. and Brumbley, S.M. (2015) Production of novel biopolymers in plants: recent technological advances and future prospects. Current opinion in biotechnology 32, 68-75.

Stark, D.M., Timmerman, K.P., Barry, G.F., Preiss, J. and Kishore, G.M. (1992) Regulation of the Amount of Starch in Plant-Tissues by Adp Glucose Pyrophosphorylase. Science 258, 287-292. 
Streb, S., Delatte, T., Umhang, M., Eicke, S., Schorderet, M., Reinhardt, D. and Zeeman, S.C. (2008) Starch granule biosynthesis in Arabidopsis is abolished by removal of all debranching enzymes but restored by the subsequent removal of an endoamylase. The Plant cell 20,3448-3466.

Streb, S. and Zeeman, S.C. (2012) Starch metabolism in Arabidopsis. The Arabidopsis book / American Society of Plant Biologists 10, e0160.

Suzuki, A., Shibanuma, K., Takeda, Y., Abe, J.-i. and Hizukuri, S. (1994) Structures and Pasting Properties of Potato Starches from Jaga Kids Purple '90 and Red ' 90 . Journal of Applied Glycoscience 41, 425-432.

Sweetlove, L.J., Burrell, M.M. and apRees, T. (1996) Starch metabolism in tubers of transgenic potato (Solanum tuberosum) with increased ADPglucose pyrophosphorylase. Biochemical Journal 320, 493-498.

Swinkels, J.J.M. (1985) Composition and Properties of Commercial Native Starches. Starke 37, 1-5.

Syahariza, Z., Sar, S., Hasjim, J., Tizzotti, M.J. and Gilbert, R.G. (2013) The importance of amylose and amylopectin fine structures for starch digestibility in cooked rice grains. Food chemistry 136, 742-749.

Tabata, S., Nagata, K. and Hizukuri, S. (1975) Studies on Starch Phosphates .3. Esterified Phosphates in Some Cereal Starches. Starke 27, 333-335.

Takeda, Y. and Hizukuri, S. (1982) Studies on Starch Phosphate .6. Location of Phosphate Groups in Potato Amylopectin. Carbohyd Res 102, 321-327.

Tan, I. and Halley, P.J. (2014) "Structure-Property" Relationships of Genetically Modified Starch. Starch Polymers: From Genetic Engineering to Green Applications, 1947.

Tanackovic, V., Svensson, J.T., Jensen, S.L., Buleon, A. and Blennow, A. (2014a) The deposition and characterization of starch in Brachypodium distachyon. Journal of experimental botany 65, 5179-5192.

Tanackovic, V., Svensson, J.T., Jensen, S.L., Buléon, A. and Blennow, A. (2014b) The deposition and characterization of starch in Brachypodium distachyon. Journal of experimental botany 65, 5179-5192.

Tester, R.F., Qi, X. and Karkalas, J. (2006) Hydrolysis of native starches with amylases. Anim Feed Sci Tech 130, 39-54.

Tjaden, J., Schwoppe, C., Mohlmann, T., Quick, P.W. and Neuhaus, H.E. (1998) Expression of a plastidic ATP/ADP transporter gene in Escherichia coli leads to a functional adenine nucleotide transport system in the bacterial cytoplasmic membrane. Journal of Biological Chemistry 273, 9630-9636.

Topping, D.L., Morell, M.K., King, R.A., Li, Z.Y., Bird, A.R. and Noakes, M. (2003) Resistant starch and health Himalaya 292, a novel barley cultivar to deliver benefits to consumers. Starch-Starke 55, 539-545.

Umemoto, T., Yano, M., Satoh, H., Shomura, A. and Nakamura, Y. (2002) Mapping of a gene responsible for the difference in amylopectin structure between japonica-type and indica-type rice varieties. Theoretical and Applied Genetics 104, 1-8.

Untergasser, A., Nijveen, H., Rao, X., Bisseling, T., Geurts, R. and Leunissen, J.A. (2007a) Primer3Plus, an enhanced web interface to Primer3. Nucleic acids research 35, W71-74.

Untergasser, A., Nijveen, H., Rao, X., Bisseling, T., Geurts, R. and Leunissen, J.A.M. (2007b) Primer3Plus, an enhanced web interface to Primer3. Nucleic acids research 35, W71-W74.

van der Krol, A.R., Mur, L.A., Beld, M., Mol, J.N. and Stuitje, A.R. (1990) Flavonoid genes in petunia: addition of a limited number of gene copies may lead to a suppression of gene expression. The Plant cell 2, 291-299.

VandenKoornhuyse, N., Libessart, N., Delrue, B., Zabawinski, C., Decq, A., Iglesias, A., Carton, A., Preiss, J. and Ball, S. (1996) Control of starch composition and structure through substrate supply in the monocellular alga Chlamydomonas reinhardtii. Journal of Biological Chemistry 271, 16281-16287.

Vikso-Nielsen, A., Blennow, A., Jorgensen, K., Kristensen, K.H., Jensen, A. and Moller, B.L. (2001) Structural, physicochemical, and pasting properties of starches from potato plants with repressed r1-gene. Biomacromolecules 2, 836-843.

Visser, R.G., Stolte, A. and Jacobsen, E. (1991) Expression of a chimaeric granule-bound starch synthase-GUS gene in transgenic potato plants. Plant molecular biology 17, 691-699.

Visser, R.G.F., Suurs, L.C.J.M., Bruinenberg, P.M., Bleeker, I. and Jacobsen, E. (1997) Comparison between amylosefree and amylose containing potato starches. Starch-Starke 49, 438-443.

Waliszewski, K.N., Aparicio, M.A., Bello, L.A. and Monroy, J.A. (2003) Changes of banana starch by chemical and physical modification. Carbohyd Polym 52, 237-242. 
Wang, Z.Y., Chen, X.P., Wang, J.H., Liu, T.S., Liu, Y., Zhao, L. and Wang, G.Y. (2007) Increasing maize seed weight by enhancing the cytoplasmic ADP-glucose pyrophosphorylase activity in transgenic maize plants. Plant Cell Tiss Org 88, 83-92.

Warren, F.J., Zhang, B., Waltzer, G., Gidley, M.J. and Dhital, S. (2015) The interplay of a-amylase and amyloglucosidase activities on the digestion of starch in in vitro enzymic systems. Carbohyd Polym 117, 192-200.

Wattebled, F., Dong, Y., Dumez, S., Delvalle, D., Planchot, R., Berbezy, P., Vyas, D., Colonna, P., Chatterjee, M., Ball, S. and D'Hulst, C. (2005) Mutants of Arabidopsis lacking a chloroplastic isoamylase accumulate phytoglycogen and an abnormal form of amylopectin. Plant physiology 138, 184-195.

Weber, C.J., Haugaard, V., Festersen, R. and Bertelsen, G. (2002) Production and applications of biobased packaging materials for the food industry. Food Addit Contam 19, 172-177.

Wei, C.X., Qin, F.L., Zhu, L.J., Zhou, W.D., Chen, Y.F., Wang, Y.P., Gu, M.H. and Liu, Q.Q. (2010) Microstructure and Ultrastructure of High-Amylose Rice Resistant Starch Granules Modified by Antisense RNA Inhibition of Starch Branching Enzyme. Journal of agricultural and food chemistry 58, 1224-1232.

Werij, J.S., Furrer, H., van Eck, H.J., Visser, R.G.F. and Bachem, C.W.B. (2012) A limited set of starch related genes explain several interrelated traits in potato. Euphytica 186, 501-516.

Worby, C.A., Gentry, M.S. and Dixon, J.E. (2006) Laforin, a dual specificity phosphatase that dephosphorylates complex carbohydrates. The Journal of biological chemistry 281, 30412-30418.

Wu, A.C., Morell, M.K. and Gilbert, R.G. (2013) A parameterized model of amylopectin synthesis provides key insights into the synthesis of granular starch. PloS one 8, e65768.

Xu, X., Visser, R.G.F. and Trindade, L.M. (2014) Starch Modification by Biotechnology: State of Art and Perspectives. In: Starch Polymers: From Genetic Engineering to Green Applications pp. 79-104. Elsevier B.V.

Yu, T.S., Kofler, H., Hausler, R.E., Hille, D., Flugge, U.I., Zeeman, S.C., Smith, A.M., Kossmann, J., Lloyd, J., Ritte, G., Steup, M., Lue, W.L., Chen, J. and Weber, A. (2001a) The Arabidopsis sex 1 mutant is defective in the R1 protein, a general regulator of starch degradation in plants, and not in the chloroplast hexose transporter. The Plant cell 13, 1907-1918.

Yu, T.S., Kofler, H., Hausler, R.E., Hille, D., Flugge, U.I., Zeeman, S.C., Smith, A.M., Kossmann, J., Lloyd, J., Ritte, G., Steup, M., Lue, W.L., Chen, J.C. and Weber, A. (2001b) The Arabidopsis sex 1 mutant is defective in the R1 protein, a general regulator of starch degradation in plants, and not in the chloroplast hexose transporter. The Plant cell 13, 1907-1918.

Yu, T.S., Zeeman, S.C., Thorneycroft, D., Fulton, D.C., Dunstan, H., Lue, W.L., Hegemann, B., Tung, S.Y., Umemoto, T., Chapple, A., Tsai, D.L., Wang, S.M., Smith, A.M., Chen, J. and Smith, S.M. (2005) alpha-Amylase is not required for breakdown of transitory starch in Arabidopsis leaves. The Journal of biological chemistry $\mathbf{2 8 0}$, 9773-9779.

Zeeman, S., Chen, J., Gatehouse, J., Smith, A. and Smith, S. (2007a) The pathways and control of starch breakdown in Arabidopsis thaliana. Photosynth Res 91, 237-237.

Zeeman, S. and Rees, T.A. (1999) Changes in carbohydrate metabolism and assimilate export in starch-excess mutants of Arabidopsis. Plant, cell \& environment 22, 1445-1453.

Zeeman, S.C., Kossmann, J. and Smith, A.M. (2010) Starch: its metabolism, evolution, and biotechnological modification in plants. Annual review of plant biology 61, 209-234.

Zeeman, S.C., Northrop, F., Smith, A.M. and Rees, T. (1998) A starch-accumulating mutant of Arabidopsis thaliana deficient in a chloroplastic starch-hydrolysing enzyme. The Plant journal : for cell and molecular biology 15, 357-365.

Zeeman, S.C., Smith, S.M. and Smith, A.M. (2007b) The diurnal metabolism of leaf starch. The Biochemical journal 401, 13-28.

Zeeman, S.C., Tiessen, A., Pilling, E., Kato, K.L., Donald, A.M. and Smith, A.M. (2002) Starch synthesis in Arabidopsis. Granule synthesis, composition, and structure. Plant physiology 129, 516-529.

Zhang, H., Liu, X., Liu, J., Ou, Y., Lin, Y., Li, M., Song, B. and Xie, C. (2013) A novel RING finger gene, SbRFP1, increases resistance to cold-induced sweetening of potato tubers. FEBS letters $\mathbf{5 8 7}, \mathbf{7 4 9 - 7 5 5 .}$

Zrenner, R., Salanoubat, M., Willmitzer, L. and Sonnewald, U. (1995) Evidence of the crucial role of sucrose synthase for sink strength using transgenic potato plants (Solanum tuberosum L.). The Plant Journal 7, 97-107. 



\section{Summary}

Storage starch is an energy reservoir for plants and the major source of calories in the human diet. Starch is used in a broad range of industrial applications, as a cheap, abundant, renewable and biodegradable biopolymer. However, starch needs to be modified before it can fulfill the required properties for specific industrial applications. Genetic modification of starch, as a green technology with environmental and economic advantages, has attracted increasingly attention. Many achievements obtained from earlier studies have demonstrated the feasibility and potential of using this approach to produce starches with novel properties (Chapter 2).

The main objective of this research was to produce novel starches with enhanced functionalities through genetic modification, while gaining a better understanding of storage starch biosynthesis. A focus on potato was warranted as it represents a superior model system for storage starch biosynthesis studies and for the production of starches with novel properties. To this end, a number of enzymes from various sources have been expressed in potato tubers to modify starch phosphate content and polysaccharide structure, since these two characteristics have long been recognized as key features in starch properties.

To modify starch phosphate content and explore starch (de)phosphorylation, a human phosphatase enzyme named laforin, and modifications of it, were introduced into potato (Chapter 3). Interestingly, modified starches exhibited a significantly higher phosphate content rather than the expected lower phosphate content. Transcriptome analysis showed that the increase in phosphate content was a result of upregulation of starch phosphorylating genes, which revealed a compensatory response to the loss of phosphate content in potato starch. Furthermore, the increase of phosphate content in potato starch was reached to a threshold level. This was in line with the observations in the modified starches from overexpressed- Glucan water dikinase (GWD1) transgenic plants (Chapter 4). Furthermore, overexpression of two starch dikinases from Arabidopsis thaliana, glucan water dikinase 2 and 3 (AtGWD2 and AtGWD3), did not result in a significant increase in phosphate content of potato starch (Chapter 5 ). Taken together, these results indicated that phosphate content of potato starch is under strict control.

Morphological analysis of starch granules containing different levels of phosphate content confirmed the indispensible role of phosphate content in the normal formation of starch granules, since cracked granules were observed in the starches containing low phosphate content, while irregular bumpy shaped granules were observed in the tubers from plants containing high phosphate content. Interestingly, further analyses on the expression level of genes involved in starch metabolism and sugar-starch conversion suggested that starch phosphorylation might affect starch synthesis by controlling the carbon flux into 
starch while simultaneously modulating starch-synthesizing genes. Further studies are needed to confirm this finding (Chapter 4).

To produce starches with novel structures, an (engineered) 4, 6-a-glucanotransferase (GTFB) from Lactobacillus reuteri 121 was introduced into potato tubers (Chapter 6). The resulting starches showed severe changes in granule morphology, but not in starch fine structure. Transcriptome analysis revealed the existence of a self-repair mechanism to restore the regular packing of double helices in starch granules, which possibly resulted in the removal of novel glucose chains potentially introduced by the (engineered) GTFB.

This research successfully generated starches with various functionalities, including altered gelatinization characteristics (Chapter 3 and 4), improved freeze-thaw stability (Chapter 4) and higher digestibility (Chapter 6). The exploitation of relationships between starch characteristics and starch properties revealed that starch properties represent the outcome of the combined effect of many factors and are highly dependent on the genetic background in which the modification has been performed.

In conclusion, the research described in this thesis demonstrates the great potential of genetic modification in producing starches with novel properties. Meanwhile, these results revealed the presence of complex and exquisite molecular regulation mechanisms for starch biosynthesis in potato. In future research, these regulations need to be taken into account for the relational design of starch in planta. Certainly, a better understanding of the process of starch metabolism in storage organs would be a great step forward towards tailoring starch in an economically important crop such as potato. 


\section{Acknowledgements}

While writing down these important words, beautiful scenes of the past years crowded in on me, sneak out of my eyes and roll down my cheeks. The scenes filled with laughter and tears, mistakes and successes; scenes filled with inspirations and motivations, challenges and excitements; scenes that help me remember the people that have come along my way and brighten up my life-journey. All of this would not have been so lovely without the contribution from all of you. Now, when I look into mirror, I see strength, courage, lessons learned, and pride in myself. This moment would not have been happened without the support and dedication of all of you.

Dr. Luisa Trindade, words cannot express all that I wish to say to you. I could not have imagined having a better supervisor for my PhD study. Luisa, thank you for the extensive knowledge you have shared with me along with your shining smile, constant helpfulness and endless kindness. You made working with you an interesting and lifetime experience. Your encouragement and patience always make me feel solid and definite. Your trust and guidance allowed me the room to work in my own way and inspired me to pursue my goals with hard work and dedication. To me, you are less of a supervisor but more of a very good friend. I remember each time I knocked on your door with confusions of life and closed the door with a big smile on my face. I remember all the hugs you gave to me whenever and wherever I needed. I remember a ton of giggles and tears with our girly talks. From my bottom of my heart, thank you so much for all of this! I will always strive to emulate you!

Prof. Richard Visser, thank you for accepting me into Plant Breeding department. Thank you for your aspiring guidance, invaluably constructive criticism and thoughtful advice during the entire process. I am sincerely grateful to you for sharing illuminating views on a number of issues related to the project. I highly appreciate all the prompt responses to all questions, especially during the writing stages of this thesis, despite the immense task of leading the whole department and being the Dean of Research of the university. Again, thank you very much!

I have been privileged to experience first-hand how passionate, kind and critical mentors can make a difference. Prof. Conghua Xie and Prof. Jun Liu, if it wasn't for you I would never be the person I am today. Your open-mindedness, encouragement and support opened the door for me, allowing all the possibilities come into my life - so excited, so unexpected and so supernaturally wonderful! Your immense knowledge, generosity, meticulous attitude and genial personality were deeply rooted in my heart, which is imparting continuously and positively on every single step of my professional and personal life. I couldn't feel luckier and more grateful. I sincerely thank you for walking ahead me whenever I needed guidance and thank you for walking behind me whenever I needed someone to watch my back. 


\section{We fought the same battle together! Friendship makes me stronger every day!}

Shairul Izan, my BFF, we made it! We started this special journey together and now we will finish it together! You have been such a terrific and constant companion no matter whether my day is sunny or rainy. In our journey, we climbed steep "mountains" hand in hand; we cheered up one another by our "decent - indecent" talks and silly jokes; we encouraged one another and built each other up. Nothing can compare to the pain of a bellyache from laughing too hard with you... or from eating too much spicy food together. I am so proud of you and all you've accomplished - be a doctor as well as an awesome mum!

Peter (Quy Dung Dinh), my sunshine-buddy, you brought sunshine to everyone with your wit, positive attitude and kindness. You were the first foreigner I talked to at our department, since then you have been always the one whom I enjoy talking to and sharing my thoughts with. You are such a great friend who I can always ask for favours and never expect "no" as an answer. We fought shoulder to shoulder in various heavy battles as "Plant-attackers", "Dream-catchers", "Naked-plants" and "Writing-buddies" and always won countless happiness and precious memories. Thank you for fighting all of these battles with me together!

Tim van der Weijde, my desk-neighbour, as you said, we've been through the challenging period of our PhD journey together! We encouraged and motivated each other by cheering for any tiny progresses almost everyday. You have guided me throughout this final stage, as you were one step ahead of me and you are always willing to give me valuable advice in this regard based on your experience. Your high-efficiency in writing has put positive pressure on me and kept me sharp and motivated to finish. Your critical thinking has inspired me to look at questions from different angles. Timmy, you are a funny and joyful person. I enjoyed very much your accompany at coffee and lunch breaks, courses and meetings, sports events... I will never forget your funny screams from roller coaster and your blunt criticism on my "best-ever" cooking... thank you for all, my great friend!

\section{Many friends and colleagues make my each single day special!}

Juan Du, if it weren't for you I probably would not have come to Wageningen so smoothly. Your thoughtfulness made me start this journey with ease. I admire your spirit of "no sooner said than done" and your capability to make complex things simple. I couldn't thank you enough. Kayleigh van Til, my awesome Dutch girlfriend, I knew you as my student, but we end up with becoming good friends. Probably it is because we are "crazy" in the similar way. I've never met someone like you, who is incredibly passionate about life. You are independent, caring and determined. You always do the best that you can and strive for the best performance that you can get, which makes me so proud of you. Thank you for bringing uncountable laughs in my life! Dália Rosa Alves Carvalho, I feel joyful and energetic every time when I see you. You have enormous talent in imparting remarkable positive energy to those around you. Thank you for all delicious Portuguese cuisine, all our 
pleasant chats and all self-torture time spent together in the gym. Alessandra Fracasso, when I think back, the time when you were here probably was the busiest time of my PhD life. Although it was a short period, we have done a lot of fun things together: gliding, cooking, dancing, exercising, meeting friends and etc. I remember that we couldn't bear to say goodbye every time when you are going back to Italy, we always say "see you soon" with tears in our eyes. Ale, I miss you!

Jordi Petit, my little brother, if you were not in our office, it wouldn't have had such a happy atmosphere. I just realize that we have quite some common interests. We both like growing plants, together we made office a jungle; we both like decorating office for special events; we both like doing painting... No wonder we have so much to talk and continuous tiffs! Thank you for all the enormous fun! Mathilde Daniau, we are so different but somehow we got connection. The more I know about you, the more impressed I get. I am impressed by your inner strength, dedication and your kindness. Thank you for all your careness and all the help! Viviana Jaramillo, do you believe that sometimes when you meet a person and you just simply click? I believe it! And I believe our friendship was born right at that moment! You are an amazingly sweet and smart girl. I met you just right at the most challenging time, the writing phase. Your supports and encouragements have accompanied me along the way, which greatly helps me get to where I am today. I really appreciate your willingness to help out wherever needed!

My sincere appreciation is extended to all people that help me in all aspects of my project. This thesis couldn't be finished without their scientific discussion, remarkable assistance and excellent performance. Luc Suurs, thank you for introducing me to starch-related work in the biochemical lab. Your knowledge and assistance greatly helped me understand my project. Your patience and hearty laugher will always be remembered and appreciated. Annemarie Dechesne, it was a great pleasure to work with you. Your effort made lots of new trying possible and always kept the work on schedule. You ability to multitask, analytical skills and problem solving abilities have really impressed me. Thank you very much for all the help and enjoy every moment of your new chapter of life with your little princess! Heleen Furrer, I like working together with you and listening to your special radio channel. I also like when you show your travel or family photos and share your stories with me. You are always kind, sweet and patient with me. Dear Heleen, thank you for all your help and all little sweets! Dankjewel voor alle nederlandse lessen. Dianka Dees, you gave me help at the moment I needed it most. Thank you for investing time to help my work and to listen to my grumps at each difficult time. Thank you for your kindness and all the enjoyable conversations we had. Isolde, Marjan, Iris and Bernadette, every time when I walk into your office, sweet air always greets me. Your closeness and creativity made the room bright and shining. Your kindness and cheerfulness made the tissue culture become the most relaxing work to me. Dirkjan Huigen, I highly appreciate your help for taking care of the plants in the greenhouse. Abe, Manos and Thamara Hesselink, thank 
you for your patience and help on protein analysis. Patrick, thank you for the microscopy trainings. Anne Kortstee, thank you for fruitful discussions and your scientific knowledge. Gerard Bijsterbosch, thank you for all the practical solutions you provided when facing any technical problem. Xing-Feng Huang, thank you for all the information regarding the project and comments of the manuscripts. Andres, Rene, Elma and Robert, thank you for all comments and suggestions on my work during seminars or group meetings. Christel, Linda, Irma, Fien, Jos, Brian, Gert, Doret, Danny, Koen, Marcel and Annelies, thank you all for the always-available help and laughs in the molecular lab.

I would like to thank those who have made my life in Wageningen an unforgettable experience. Firstly, I would like to thank all my Chinese friends, with whom I celebrated dinners, birthday parties, graduation ceremonies, Wageningen Olympics, holiday trips and many more. Thank you for making life in the Netherlands feel more like home. My special thanks to Li Ying, Zheng Zheng, Cheng Jihua, Qi Weicong, Chen Xi, Zhang Zhao, Lin Ke and Zhang Ningwen, who lighten up my path with their experience. Sun Kaile, we came to a tacit agreement to perform "Liu Kou Cha". We should keep up with it! Wang Yanli, my travel buddy, we had so many adventures together and each of them leaves me beautiful memories. Li Huchen and Song Wei, my diving buddies, the time we spent in diving was unforgettable. Promise me! At least once we go together and explore the mystery of a beautiful sea. Lin Xiao, Xiao Tingting, Zeng Tian, Zhang Jianhua, Bai Bing, Huang Pingping, Cheng Xu, Zhu Feng, Wang Shuhang, Bai Yuling, Wang Xingxing, Yao Mingtian, Song Chunxu, He Wei and Sun Xiaoxue, thank you very much for helping me out whenever I needed it.

Moreover, I would also like to thank all my other friends and colleagues. Nicole, Janneke and Letty, you have spoiled me with your loving attitude and handy help. Thank you for lovely talks and numerous laughter. Guusje, I have only recently started getting to know you better. Your patience, extensive knowledge and thoughtfulness are admirable. Thank you for your believing in me! Aurelie, Anne Giesbers, Myluska, Mas, Michela, Peter Bourke, Charlie, Clarie, Vivianne, Marine, Narges, Kim, Carolina, Valentina, Cynara, Sara, Marcela, Rafael, Marian, Christos, Ernest, Jast, Jan, Alex, Henk, Erik, Anne-Marie Wolters and many many others, thank you for being source of support and laughter during these years. Your companies have made my life incredibly colorful!

致我最爱的爸爸妈妈, 感谢您们对我照顾、爱护和默默付出; 感谢你们在我成长道 路上的理解、支持和宽容; 感谢你们的信任和无私, 让我自由摸索属于我自己的道 路。女儿虽不经常待在你们身边, 但是心中无时不在牵挂着你们, 祝愿你们身体健 康, 万事如意!

To my Johan, thank you for being by my side along this journey, and thank you for always giving me reasons to cheer. Your understanding, patience and support truly made this journey much easier for me. Your "very funny" jokes, sweet smiles and tender careness 
made this journey even more enjoyable. Along the way, worries were halved because I can share them with you; happiness was doubled because you never let me feel blue.

There are many more people I would like to thank. I may have missed your names here, but you are always remembered and appreciated.

Wageningen, September 2016 



\section{About the author}

Xuan Xu was born on January $13^{\text {th }}, 1986$ in Enshi, Hubei, China. In 2004, Xuan moved to Wuhan to study at Huazhong Agriculture University, where she graduated with distinction and obtained BSc degree in horticulture. In the last two years of bachelor study, Xuan focused on vegetable breeding and further raised her interest in improving the quality of vegetables by using biotechnology. Therefore, Xuan continued her master's study in "Molecular Biology and Biotechnology" in the same university, where she focused on improving the processing quality of potatoes and developing intragenic transformation tools. After two years of study, she was offered an opportunity to

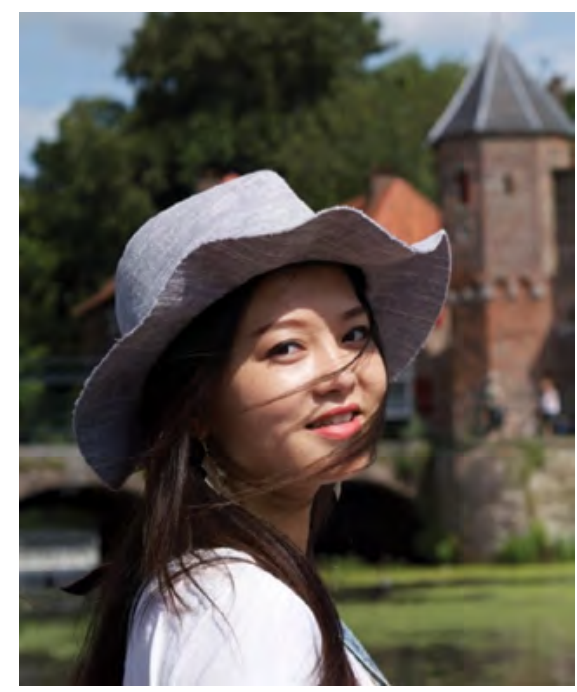
join "combined mater-doctor program" and pursue doctoral degree directly. However, she decided to study abroad and further deepen her knowledge. In 2011, following the successful scholarship application from China Scholarship Council, Xuan started her PhD study on modification of starch composition and functionalities using biotechnology under the supervision of Dr. Luisa Trindade and Prof. Richard Visser at the laboratory of Plant Breeding of Wageningen University \& Research in the Netherlands. In this thesis, the results obtained during her PhD period are presented. 



\section{Education Statement of the Graduate School Experimental Plant Sciences}

Issued to: Xuan Xu

Date: $\quad 14$ October 2016

Group: Laboratory of Plant Breeding

University: Wageningen University \& Research

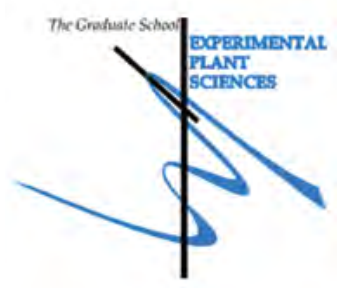

1) Start-up phase

$\underline{\text { date }}$

\section{- First presentation of your project}

Modification of potato starch granule by expression of granule-bound fusion

Oct 23, 2012 proteins

- Writing or rewriting a project proposal

Modification of potato starch granule by expression of granule-bound fusion

Oct 2012 proteins

- Writing a review or book chapter

Chaper 4 'Starch modification by biotechnology: state of art and perspectives',

Jun 2012

IN Starch Polymers (Eds. Halley \& Averous), Elsevier, Nov 2014, p. 79.

- MSc courses

Laboratory use of isotopes

Subtotal Start-up Phase

13.5 credits*

2) Scientific Exposure

date

- EPS PhD student days

EPS PhD student day, Leiden, NL

Nov 29, 2013

EPS 'Get2Gether' event, Soest, NL

Jan 29-30, 2015

- EPS theme symposia

EPS Theme 3 Symposium "Metabolism and Adaptation", Utrecht University

Apr 26, 2013

EPS Theme 3 Symposium "Metabolism and Adaptation", Wageningen University

Mar 11, 2014

\section{- Lunteren days and other National Platforms}

Annual Meeting “Experimental Plant Sciences”, Lunteren, NL

Apr 02-03, 2012

100 Years Plant Breeding: Open Day, Wageningen, NL

Aug 31, 2012

2nd starch round table 2012, Wageningen, NL

Nov 28, 2012

Annual Meeting “Experimental Plant Sciences”, Lunteren, NL

Apr 22-23, 2013

Mini-symposium 'Developments in carbohydrate analysis', Amsterdam, NL

Nov 15, 2013

3 rd starch round table 2014, Wageningen, NL

Nov 26, 2014

Annual Meeting “Experimental Plant Sciences”, Lunteren, NL

Apr 13-14, 2015

- Seminars (series), workshops and symposia

EPS - Mini-symposium 'Plant Breeding in the genomics era'

Nov 25, 2011 
Plant Breeding research day 2012

Feb 28, 2012

Mini-symposium 'Cisgensis, nextstep in classical plant breeding?'

Jun 14,2012

Invited seminar' Opportunities for conservation genetics with genome wide

Sep 07, 2012

information'

Workshop 'Make more business with your research'

Apr 23, 2013

Workshop 'Advanced Light Microscopy facilities at Wageningen UR'

Jun 13,2013

WGS PhD Workshop Carousel 2014

Jun 02, 2014

Invited seminar 'New directions in Potato Breeding and Quantitative Genetics

Jun 26,2014

at the University of Wisconsin'

Plant Breeding research day 2014

Sep 30, 2014

Workshop'JoinMap course'

May 18, 2016

Invited seminar 'Rice nutritional improvement through modification

Jun 21, 2016

of aleurone development'

\section{- Seminar plus}

\section{- International symposia and congresses}

Next generation plant breeding conference

Nov 11-14, 2012

5th Joint $\mathrm{PhD}$ retreat, Gent

Jul 23-26, 2013

Symposium 'All-inclusive breeding: Integrating highthroughput science'

Oct 16, 2014

The Starch Convention 2015, Detmold, Germany

Apr 15-16, 2015

The 11th Carbogydrate Bioengineering Meeting, Espoo, Finland

May $10-13,2015$

\section{- Presentations}

Poster: 5th Joint PhD retreat, Gent

Jul 23-26, 2013

Oral: EPS Theme 3 symposium, Wageningen

Mar 11, 2014

Oral: ALW Meeting Experimental Plant Sciences 2015, Lunteren

Apr 14, 2015

Poster: The 11 th Carbogydrate Bioengineering Meeting, Finland

May 10-13, 2015

\section{$-\quad$ IAB interview}

- Excursions

Subtotal Scientific Exposure

15.1 credits* $^{*}$

3) In-Depth Studies

date

- EPS courses or other PhD courses

Bio-energy production from crop plant and algae

Nov 21-23, 2012

PhD Course 'Bioinformatics - a User's Approach'

Mar 04-08, 2013

13th Summer Course Glycosiciences

Apr 13-17, 2014

- Journal club

- Individual research training 
\begin{tabular}{|lr|}
\hline 4) Personal development & $\underline{\text { date }}$ \\
Skill training courses & Oct-Dec 2014 \\
Scientific Writing & May 19, 20, 26 and 27, 2014 \\
The Essentials of Scientific Writing and Presenting & Jun 02, 2014 \\
WGS PhD Workshop Carousel 2014 & Sep 29-30, 2015 \\
Adobe InDesign Essential Training & Oct 2013-Jan 2014 \\
Social dutch for Employees & Sep 25, 2015 \\
Workshop'Last Stretch of the PhD Programme' & \\
Organisation of PhD students day, course or conference & Sep 2014-Sep 2015 \\
Monday seminar of Plant Breeding department & \\
Membership of Board, Committee or PhD council &
\end{tabular}

Subtotal Personal Development

7.5 credits $^{*}$

TOTAL NUMBER OF CREDIT POINTS*

40.5

Herewith the Graduate School declares that the PhD candidate has complied with the educational requirements set by the Educational Committee of EPS which comprises of a minimum total of 30 ECTS credits

${ }^{*}$ A credit represents a normative study load of 28 hours of study. 
The project was financed by the China Scholarship Council (CSC) and the Laboratory of Plant Breeding, Wageningen University \& Research, The Netherlands. Financial support from Wageningen University for printing this thesis is gratefully acknowledged.

Cover \& layout design : lliana Boshoven-Gkini | AgileColor.com

Printed by: GVO drukkers \& vormgevers, Ede (NL) | gvo.nl 


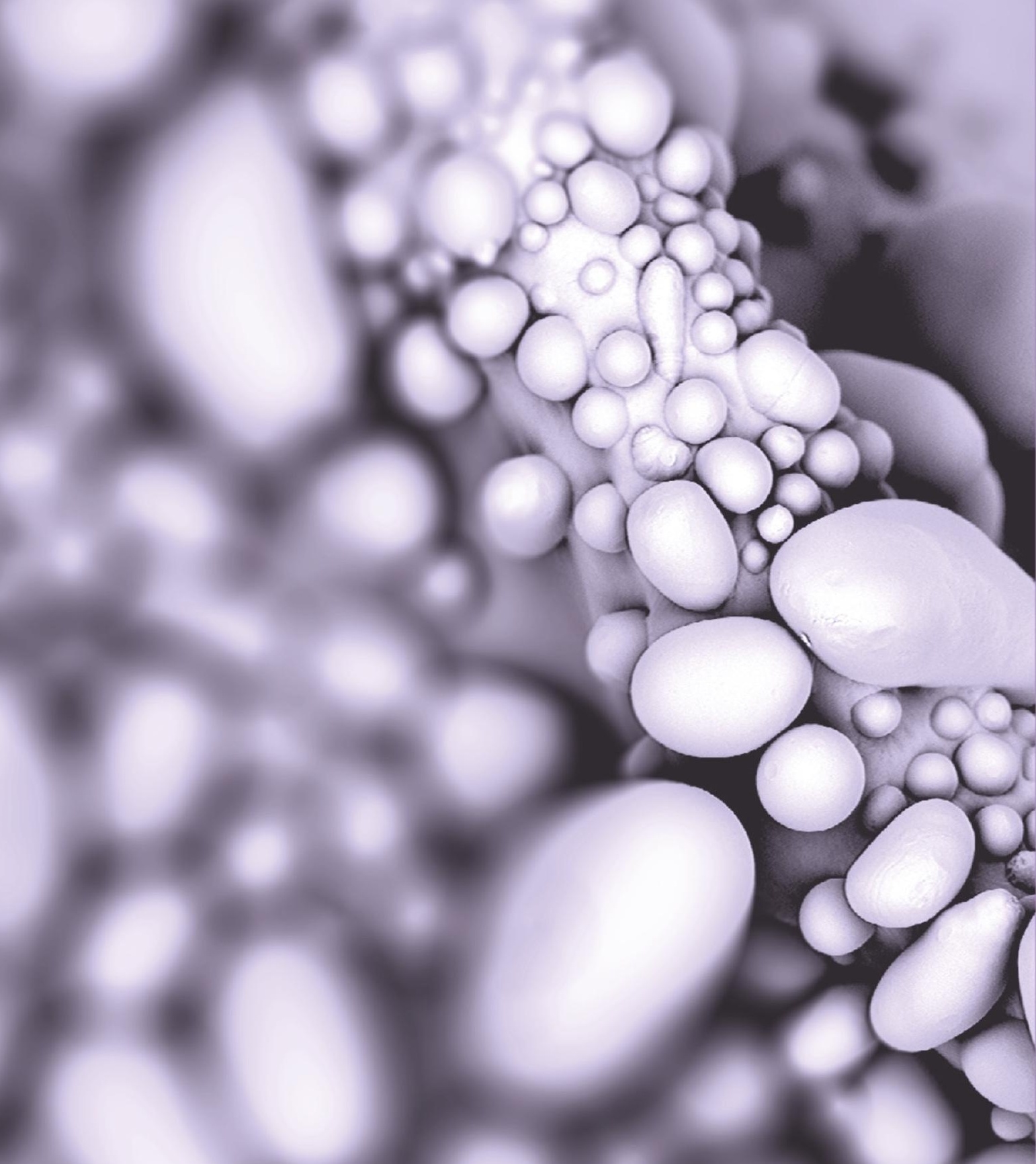

